This report is derived from a dissertation submitted to the Graduate School, University of Texas, Austin, TX, in partial fulfillment of the requirements for the Degree of Doctor of Philosophy.

This report represents the independent work of the author and has not been edited by the Technical Information staff.

Printed in the United States of Amorica. Available trom National Technical Information Service

U 5 Department of Commerce

5285 Port Royal Road

Springtield, VA 22151

Price: Printed Copy $\$ 7.60$ Microfiche $\$ 2.25$

Thin roport was preciared as an secount of work sponsored by the United Sintes Govenninent. Nelther the Uniesd State: nor the United States Everny Rewearch and Development Administration, nor any of their employes, mor any of their conwarrentiv, express or implied, or apsumpes any legilliablity or rever informe tion

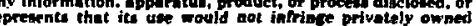
represench
vients. 
Page

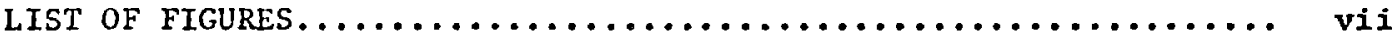

LIST OF TABLES................................. xii

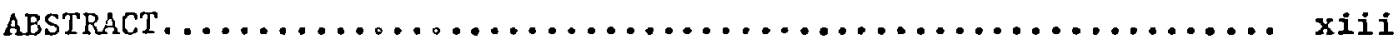

I. INTRODUCTION............................. 1

1.1) Monte Carlo Development..................... 2

1.2) Incentive for this Research................... 6

1.3) Pattern Recognition....................... 8

1.4) Purpose of Dissertation...................... 9

1.5) Scope of Dissertation...................... 10

1.6) Outline of Dissertation...................... 11

II. THE MONTE CARLO METHOD AND V.ARIANCE REDUCTION........... 13

2.1) The Nonte Carlo Method for Particle Transport........ 13

2.1.1) Basic Principles.................... 13

2.1.2) Estirate of Statistical Errors............ 19

2.2) Variance Reduction........................ 22

2.3) Survey of Variance Reduction Techniques............. 26

2.3.1) Splitting and Russian Roulette.......... 27

2.3.2) Other Techniques...................... 29

2.3.3) Variance Reduction Through Learning......... 31

2.4) Splitting and Russian Roulette in State Space........ 33

2.5) Effectiveness of Variance Reduction Techniques........ 34

III. PATTERN RECOGNITION.......................... 37

3.1) The General Pattern Recognition Problem............ 38

3.1.1) Fundamentals...................... 38 
Page

3.1.2) Types of Pattern Classifiers.............. 46

3.2) Sequential Deterministic Classification Techniques.... 50

3.2.1) Linear Discriminant Functions............ 50

3.2.2) Quadratic Discriminant Functions........... 59

3.3) Sequential Statistical Classification Techniques...... 60

3.3.1) Linear Discriminant Functions............. 61

3.3.2) Quadratic Discriminant Functions........... 66

3.4) Feature Selection.......................... 66

3.5) Multiclass Problems........................ 68

IV. RECOGNITION OF SPLITTING SURFACES................. 71

4.1) Basic Principles........................ 72

4.1.1) Prototypes from Monte Carlo Calculations..... 73

4.1.2) Feature Selection.................. 78

4.1.3) The Pattern ClassLfier................. 79

4.1.4) The Learning Parameter, $\lambda . \ldots \ldots \ldots \ldots . \ldots . \ldots . . . . .81$

4.1.5) Classifier Performance................. 84

4.2) One Dimensional, One Region Slab................ 88

4.2.1) Deterministic Techniques................ 94

4.2.2) Statistical Techniques................. 101

4.2.3) Comparison of Deterministic and Statistical Techniques......................... 118

4.3) Multi-Region $S l a b$ and Initial Conditions........... 125

4.3.1) Initial Conditions.................. 130

4.3.1a) Deterministic Classifier.......... 130

4.3.1b) Statistical Classifier with Loss $=$ d. $\quad 136$

4.3.1c) Statistical Classifier with Loss = D. 145 
4.3.1d) summary................... 152

4.3.2) Multi-Rcgion Slab Variations.............. 153

4.4) Distance and Angle, A Two Dimensional Problem........ 159

4.4.1) Deterministic Classifier................ 168

4.4.2) Statistical Classifier................. 168

4.4.3) Sumary........................ 172

4.5) Sumnary.............................. 174

V. DISCUSSION OF GENERAL APPLICATIONS.................... 179

5.1) Implementation......................... 179

5.1.1) Single Taliy...................... 180

5.1.2) Multiple Tallies................... 188

5.1.3) The Point Detector Tally............... 189

5.2) Feature Selection.......................... 192

5.3) Timing Consjderations and Effectiveness........... 201

5.3.1) State Space Vs. Conventional Splitting...... 202

5.3.2) Time Spent for Pattern Recognition......... 209

5.3.3) Effectiveness....................... 222

5.4) Limitations........................... 227

5.4.1) Classifier Selection................ 227

$5.4 .2) \quad$ User Input........................ 228

5.4.3) Range of Application................. 229

5.5) Sumnary.............................. 230

vi. CONCLUSIONS AND RECOMELNDATIONS.................... 231

ACKNOWLEDGERENTS. ....................................... 234 APPENDICES

A Monte Carlo Sampling for Neutron Transport.......... 235

B Statistical Errors in Monte Carlo................ 267 
C FORTRAN Codjing for the One Dimensional Homogeneous slab Monte Carlo Problem.

D FORTRAN Coding for the Fractional Correction kule Deterministic Classifier..................... 275

E Derivation of $\nabla \tilde{R}$ For Various Loss Functions.......... 278

F FORTRAN Coding for the Statistical Pattern Classifier using Loss $=$ d............................. 286

G FORTRAN Coding for the One-Dimensional Multi-Region $\mathrm{S} 1 \mathrm{ab}$.

H FORTRAN Coding for the Two-Dimensional Multi-Region Slab with a Deterministic Pattern Classifier..........

I FORTRAN Coding for the Statistical Classifier with Two-Dimensional Pattern space................. 293

$J$ Geometry Description Used in the Neutron Monte Car1o

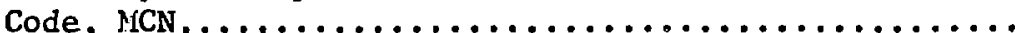

$\mathrm{K}$ Derivation of $\mathrm{S}_{j+1}$ for the Statistical Classifier with Loss $=$ d........................... 296 


\section{LIST OF FIGURES}

Figure

Page

2.1 A Probability Density Function..................... 1'

2.2 A Probability Distribution Function................ 15

2.3 Sampling Distance to Colliston................... 16

2.4 Reducing Error by Increasing the Number of Histories...... 22

2.5 Splitting Planes ir One Dimension................. 28

$2.6 \quad$ Energy splitting............................. 29

$2.7 \quad$ Source Blasing $\ldots \ldots \ldots \ldots \ldots \ldots \ldots \ldots \ldots \ldots \ldots \ldots \ldots \ldots \ldots \ldots \ldots$

2.8 Implementation of Variance Reduction Techniques......... 36

3.1 Input - Output Model of Weather Prediction ............ 38

3.2 Pattern Space ............................... 39

3.3 Inpnt - Output Modal of a Pattern Recognition System ...... 40

$3.4 \quad$ Feature space $\ldots \ldots \ldots \ldots \ldots \ldots \ldots \ldots \ldots \ldots \ldots \ldots \ldots \ldots \ldots \ldots \ldots \ldots$

3,5 Feature Selection $\ldots \ldots \ldots \ldots \ldots \ldots \ldots \ldots \ldots \ldots \ldots \ldots \ldots \ldots \ldots \ldots$

3.6 A Pattern Recognition System .................... 43

$3.7 \quad$ Decision Surface $\ldots \ldots \ldots \ldots \ldots \ldots \ldots \ldots \ldots \ldots \ldots \ldots \ldots \ldots \ldots \ldots \ldots$

3.8 Overlapping and Non-Overlapping Classes ............. 47

3.9 Linearly and Non-Linearly Separable Classes ........... 52

3.10 Weight Space $\ldots \ldots \ldots \ldots \ldots \ldots \ldots \ldots \ldots \ldots \ldots \ldots \ldots \ldots \ldots \ldots \ldots \ldots$

3.11 One Dimensional Featute Space $\ldots \ldots \ldots \ldots \ldots \ldots \ldots \ldots \ldots \ldots \ldots \ldots$

3.12 Pattern Hyperplanes in Welght Space................ 55

3.13 Example of Fixed Increment, Absolute Correction, and
Fractional Correction Rules........................ 58

3.14 Quadratic Discriminant Functions .................. 60 
3.15 Misclassification Distance.................... 62

3.16 Linearization by Feature Selection................ 67

3.17 Mult - class Problens........................ 70

4.1 Prototypes From a One Dimensional Slab Monte Carlo Problem..................................

4.2 The One Dimensional, One Region, Homogeneous Slab....... 89

4.3 Importance Distributions for Several Slab Thicknesses..... 9n

4.4 Class Distributions for Several Slab Thicknesses........ 91

4.5 Distribution of Importances at $x=0,5$, and 9 for $L=10$

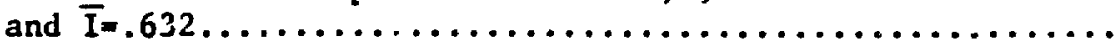

4.6 Behavior of the Decision Surface and Weight Space using the Deterministic Classifier for 100 Source Particles.....

4.7 Effect of Buffer Zones on Class Distrilutions for Several Slab Thickntsses......................

4.8 Relative Risk as a Function of Decision Surface Location for Various Loss Functions..................... 105

4.9 Misclassification Rate Vs. $\lambda$ for Various Loss Functions ... 110

4.10 Variability Vs. $\lambda$ for Various Lose Functions ........... 111

4.11 Misclassification Rate Vs. Number of Source Particles

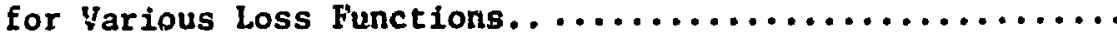

4.12 Effect of Buffer Zones on the Risk................ 114

4.13 Effect of Buffer Zones on Misclasstfication Rate and

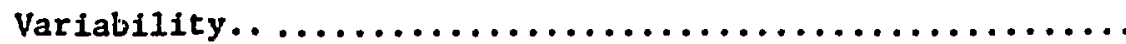

4.14 Smoothing of the Convergence Due to Buffer Zones........

4.15 Comparison of the Performance of Deterministic and Statistical Classifiers........................

4.16 Behavior of the Decision Surface.................. 120

4.17 The Multi-Region Slab Problem .................... 126

4.18 Importance Distributions for the Mul.ti-Region Slab Problem 
4.1.9 Class Distributions for the Multi-Region Slab Problem.... 129

4.20 Performance Vs. $\lambda$ for Various Initial Conditions,

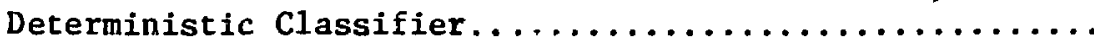

4.21 Performance Vs. Number of Source Particles for Various Initial Conditions, Deterministic Classifier...........

4.22 Behavior of $\mathrm{s}_{j+1}$ for the Deterministic Classifier.......

4.23 Behavior of $s_{j+1}$ for the Statistical classifier with

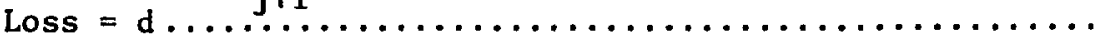

4.24 Performance Vs. $\lambda$ for Various Initial Conditions, Statistical Classifier with Loss $=\mathrm{d} \ldots \ldots \ldots \ldots \ldots \ldots \ldots \ldots$

4.25 Performance Vs. Number of Source Particles for Various Initial Conditions, Statistical Classifier with Loss $=\mathrm{D}$. .

4.26 Behavior of $\mathrm{S}_{j+1}$ for the Statistical Classifier with

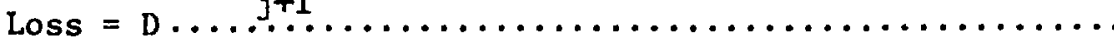

4.27 Performance Vs. $\lambda$ for Various Initial Conditions,

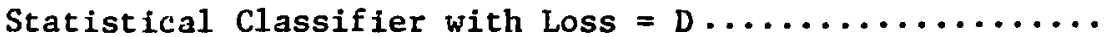

4.28 Performance Vs. Number of Source Particles for Various Initial Conditions, Statistical Classifier with Loss $=\mathrm{D}$..

4.29 Misclasification Rate Vs. $\lambda$ for Four Different MultiRegion Problems............................

4.30 Variability Vs. $\lambda$ for Four Different Multi-Region

Problems.................................

4.31 Misclassification Rate Vs. Number of Source Particles for Four Different Multi-Region Problems..................

4.32 Variability Vs. Number of Source Particles for Four

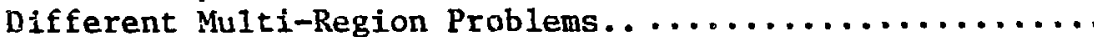

4.33 The Two Dimensional Monte Carlo Problem.............. 160

4.34 Importance and Prototype Distributions for the Two Dimensional Monte Carlo Problem....................

4.35 Class Distributions for the Two Dimensional Monte Carlo Problem................................. 164

4.36 Decision Surface for the Two Dimensional Problem ....... 166 


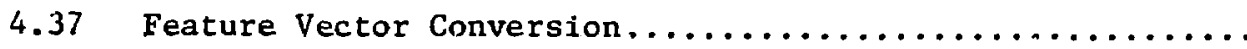

4.38 Performance Vs. $\lambda$ for the Two Dimensional Problem....... 169

4.39 Misclassification Rate Vs. Number of Source Particles for the Two Dimensional Problem.................. $17 n$

4.40 Variability Vs. Number of Source Particles for the Two Dimensional Problem.......................... 171

5.1 Saving Information for Prototypes................ 181

5.2 Communication between the Pattern Classifier and the

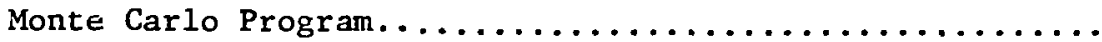

5.3 Multiple Contributions to a Tally................. 183

5.4 Iterative Procedure for Generating Splitting Surfaces,... 187

5.5 Structure of the Feature Selector................. 193

$5.6 \quad$ Cylindrical symmetry ........................ 197

5.7 Feature Selection to Avoj.d a Quadratic g(Y) .......... 197

5.8 Sample Problem for Feature Selection............... 190

5.9 Conventional Geometry Splitting.................. 204

5.10 Determining the New Class for State Space Splitting...... 206

5.11 Splitting Surfaces for the Problem of Section 4.4...... 209

A.1 Geometry Coordinates........................ 236

A.2 Major Monte Carlo Operations for Neutron Transport....... 239

A.3 Sampling Two Dimensional Sources.................. 242

A.4 Sampling a Rectangular Two Dimensional Source......... 243

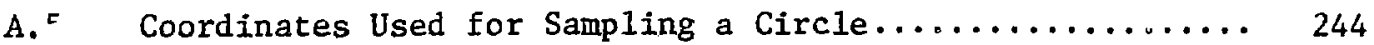

A. 6 Sampling a Circular Two Dimensional Source........... 244

A.7 Sampling by the Rejection Technique................. 245

A. 8 Right Circular Cylinder...................... 246 
A.9 Sampling the Right Circular Cylinder............. 247

A.10 The Directed Beam Source....................... 248

A.11 The Isotropic Source.......................... 249

A.12 Sampling an Isotropic Source................... 249

A.13 Linear Interpolation to Determine the Energy........... 251

A.16 Scattering in the Lab System.................. 259 
4.1 Prototypes for Problem Illustrated in Figure 4.1

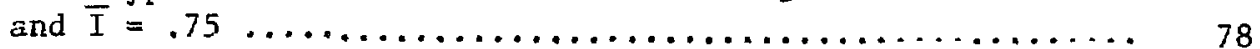

4.2 Results of $\lambda$ and $L$ Variations after 100 Source Particles using the Deterministic Classifier................. 96

4.3 Effect of Buffer Zones on the Performance of the Deterministic Classifier after 100 Source Particles....... 100

4.4 Loss Functions and $\nabla S$ Components................. 102

4.5 Timing Parameters for the One-Dimensional Problem....... 122

4.6 Multi-Region Sample Problems.................... 128

4.7 Timing Parameters for the Two-Dimensional Problem ...... 173

5.I Eight Classes Created by the First Four Splitting Surfaces. 188 


\title{
INVESTIGATION OF PATTERN RECOGNITION TECHNIQUES FOR THE IDENTIFICATION OF SPLIITING SURFACES IN MONTE CARLO PARTICLE TRANSPORT CALCULATIONS
}

by

James I. Macdonald

\begin{abstract}
Statistical and deterministic pattern recognition systems are designed to classify the state space of a Monte Carlo transport problem into importance regions. The surfaces separating the regions can be used for particle splitting and Russian roulette in state space in order to reduce the variance of the Monte Carlo tally.

Computer experiments are performed to evaluate the performance of the technique using one and two dimensional Monte Carlo problems. Additional experiments are performed to determine the sensitivity of the technique to various pattern recognition and Monte Carlo problem dependent parameters.

A system for applying the technique to a general purpose Monte Carlo code is described. An estimate of the computer time required by the technique is made in order to determine its effectiveness as a variance reduction device. It is recommended that the technique be further investigated in a general purpose Monte Carlo code.
\end{abstract}




\section{Introduction}

The Monte Carlo method of particle transport was originally developed by Fermi, Ulam, and von Neumann during the mid 1940's.' As digital computers became larger the method became more practical. Because of the large running times encountered on early computers, the method gained the reputation of being extremely time consuming. As a result, the Monte Carlo method has often been referred to as "a method of last resort."2 The current generation of digital computers, such as the $\operatorname{CDC} 7600$, has reduced the running time of problems previously taking in the hours or days to only a few minutes. As a result, for the Monte Carlo problems run today the method is hardly considered a "method of last resort" and in many cases is the "only method of resort." A description of Monte Carlo code development and the current state of the art at the Los Alamos Scientific Laboratory is given in Section 1.1 .

When the Monte Carlo method is used to solve a transport problem,the calculated answer is based on the sampling of statistical processes. Because of this, the answer has associated with it a probabilistic error based on the statistical behavior of the answer. The purpose of a Monte Carlo calculation is to calculate an answer that has a variance below some acceptable level. As a result there have been numerous techniques devised to accelerate the reduction of the variance. These techniques and their corresponding problems are discussed in Section 1.2. 
The purpose of this thesis is to allow the computer to assist in the reduction of the variance by using pattern recognition techniques. The field of pattern recognition is very new, beginning with the introduction of large computers in the 1960's. Pattern recognition is discussed briefly in Section 1.3 .

Sections $1.4,1.5$, and 1.6 describe the purpose, scope, and outline of the dissertation respectively.

\section{1) Monte Carlo Developrent}

The development of the Monte Carlo method as an accepted discipline and research tool began during the second World War from weapons development work. ${ }^{1}$ These early applications are usually attributed to the work of Fermi, von Necmann, and Ulam ${ }^{1}$ and involved the simulation of neutron diffusion in fissile material. Even at this early stage the techniques of "splitting" and "Russian roulette" were being used for variance reduction; ${ }^{3}$ however, the more rigorous development of importance sampling was performed by Harris and Herman Kahn in $1948 .^{1}$

The first open discussions of Monte Carlo applications work took place in 1949 at a symposium sponsored by the RAND Corporation. Sirce computer machinery did not exist at that time, calculations were usually performed by hand. In the course of describing the usefulness of an alignment chart for making calculations, Spinrad ${ }^{4}$ states 
...It also enables the computer to work completely on one sheet of paper, only interrupting his vision when a new randoin number is required...

where the term "computer" refers to the person performing the calculation and the random numbers were provided from tables by RAND Corporation $^{5}$. Desk top calculators helped speed up calculations some; however, these early calculators could only add, subtract, multiply, and divide.

At Los Alamos, the first semi-useful equipment for performing Monte Carlo calculations were IBM accounting machines. ${ }^{6}$ The development of MANIAC I resulted in the first computer application of Monte Carlo at LASL. ${ }^{6}$ However, each problem had to be programmed separately, in machine language. Examples of some of these early problems are given in reference 7 .

As Monte Carlo deveioped in the 50's, it quickly became a "fad" as is described in reference 1 :

...There was an understandable at tempt to solve every problem in sight by Monte Carlo, but not enough attention paid to which of these problems it could solve efficiently and which it could only handle inefficiently; and proponents of conventional numerical methods were not above pointing to those problems where Monte Carlo methods were markedly inferior to numerical analysis...

However, the same author notes when referring to the 60 's: ...In the last few years Monte Carlo methods have come back into favor. This is mainly due to better recognition of those problems in which it is the best, and sometimes the only, available technique... 
The problems for which Monte Carlo is best suited have Increased in number for the following reasons:

(1) Improved variance reduction techniques have made Monte Carlo more efficient where before it was very inefficient.

(2) Computer machinery has improved so as to make previously unsolvable problems solvable in a reasonable amount of time.

(3) Increasing demands for details to be included in a problem have in some cases eliminated solution by numerical techniques which required many simplifying assumptions. Examples of this are particle transport involving mixed diffusion and streaming effects, three-dimensional complex geometrles, and requirements for non-group energy treatments.

As a result of the demands for Monte Carlo calculations, group TD-6 of the Los Alamos Scientific Laboratory has developed a number of particle transport codes $^{8}$. Although these codes are primarily intended for weapons development, they are often used in many other programs at LASL. These computer sodes are used on CDC 7600 computers and include the following:

(1) $\operatorname{MCN}^{2}-$ A neutron transport code

(2) $M_{C G}^{3}{ }^{3}$ A gama ray transport code

(3) MCP 3 - A general photon transport code (includes lower energy treatment than MCG)

(4) MCNG - A combined neutron-gamma transport code 
(5) $\mathrm{MCNA}^{2}$ A neutron adjoint code

(6) MCK - A criticality code

(7) MCMG - A neutron-gamma multi-group transport code

(8) $\mathrm{MCGE}^{34}$ - A coupled electron-photon transport code

(9) $\mathrm{MCGB}^{3}{ }^{4}$ A gamma code with Bremsstrahlung

In the case of the neutron related codes (except MCMG) the crosssections are provided as pointwise data that is read into the codes in considerable detail. Although this greatly reduces the numir of approximations and distortions caused by cross-section reduction, it does place a consfderable burden on the computer. For example, the national Evaluated Nuclear Data File (ENDF) version of iron requires 50,000 words of storage. The codes handle three-dimensional geometry Involving first, second, and some fourth (elliptical tori) degree surfaces. All codes are progranmed in FORTRAN IV.

Although the researeh of this dissertation is applicable to Monte Carlo codes in general, it is the above sodes that are of particular interest. Thus some of the research is directly related to functions as they are performed in these codes. 


\section{2) Incentive for this Research}

The overwhelming majority of Monte Carlo improvements reported in the literature are rellated to the reduction of the probabilistic error or variance associated with the Monte Carlo answer or tally. The use of these "variance reduction" techniques varies in proportion to the difficulty encountered in their implementation. The difficulty of implementing many of these techniques is due to:

(1) The complexities involved in applying the technique to real problems. For examp.le, many of the techniques are theoretically based on very simple geometries, etc.; whereas, actual Monte Carlo problems usually involve complex three-dimensional geometries. Many of the techniques do not "scale up" to real applications.

(2) Some of the variance reduction techniques proposed are "unsafe" in that they can distort the calculations resulting in the wrong value for the tally.

(3) Most of the ceohniques require that a priori information be provided by the user. This information is usually riuantitative in nature and depends on the intuition and experience of the user. Furthermore, if the user provides the wrong information, some variance reduction techniques can actually consume more computer time than they save.

(4) Because of the diversity of Honte Carlo problems, different problems require different techniques. As a resuit, the 
user is not sure when to use one technique as opposed to another.

A fundamental problern of all variance reduction techniques is that if one were going to use a technique optimally, he would have to know all the characteristics (including the answer) of the Monte Carlo problem Deing investigated before applying the technique. Thus what is needed is a technique that instead of requiring information from the user, obtains most of the necessary inforzation during the Monte Carlo calculation. Such learningt techniques have been proposed in the literature and are discussed in Chapter II. The methods of Spanies and Mfackillañ involve learning of an optinu parameter for use with the exponential transforn technique (see Section 2.3.2). This approach has the disadvantage that it is based on a technicue, the exponential transform, which can be unsafe if used improperly. Furthermore, the technique is primerily coacerned with directional variables and is difficult to apply to problezs involving complex gecmetries. Another learning method ${ }^{12}$ has been prcposed which learns optimun spatial quantities. Besides being limited to spatial variables, this technique has difficulties with problems involving sma.II probabilities.

The most successiul techniques used in the Los Alanos Yonte Carlo codes are geometry splitting and Russian roulette (see Section 2.3.1).

* A learning technicue in this dissertation refers to the ability of a computer progran to improve its performance in solving a problem based on its own experience ${ }^{3}$. This is achieved by a preplanned strategy wherein the program modifies itself based on information gained through experience and evaluation ố its previous operations. 
These techniques are popular because they are safe and simple to implement. Even when not used optimally, they still yield large savings in computer time. These methods have the disadvantage that they are confined to spatial coordinates (energy splitting can be used, but only independently of geometry splitting) and do require that quantitative information be supplied by the user prior to implementation.

What is needed is a technique which is as safe and simple to use as splitting and Russian roulette, involves all variables of the Monte Carlo problem (spatial, directional, energy, and time) as a whole instead of independently, and relieves the user of the task of providing quantitative information. The development of such a technique using pattern recognition is the subject of this research.

\section{3) Pattern Recognition}

Before the introduction of large digital computers pattern recognition could only be described as being a human function. Examples of human pattern recognition are:

- recognition of a man from a woman

- recognition of handwritten characters

- recognition of speech

- recognition of a dog from a cat

Pattern recognition is frequently referred to as an "artificial intelligence" technique since it performs an operation on a computer which is usually considered to require intelligence. The pattern recognition 
process consists of these basic functions:

(i) identifying which features of the problem being analyzed are important

(2) finding a correlation between these features and various categories (or classes) into which the input can be sorted

(3) sorting future input into classes according to the correlation determined in (2).

These operations can be performed by mathematical transforms that usually require machine learning of some of the parameters involved. These functions will be discussed in more detail in Chapter III.

\section{4) Purpose of Dissertation}

The purpose of this dissertation is to establish a "proof of principle" for the application of pattern recognition techniques to the identification of splitting surfaces in Monte Carlo particle transport calculations. This is done by:

(1) Developing a pattern recognition system that can be used to learn splitting surfaces in Monte Carlo transport calculations.

(2) Investigating the performance of statistical and deterministic classifiers when used to recognize splitting surfaces. This investigation includes a sensitivity study of the pattern recognition parameters involved. 
(3) Proposing a system for applying pattern recognition to a general purpose Monte Carlo code.

(4) Analyzing the effectiveress that can be expected by using pattern recognition as a variance reduction technique.

Thus the purpose of this dissertation is not to apply the technique to a general purpose Monte Carlo code but to establish that such an application would be profitable.

\section{5) Scope of Dissertation}

The scope of this dissertation is limited in two areas: (1) the selection of a pattern recognition system and (2) the selection of Nonte Carlo problems used for demonstration.

There have been many pattern recognition systems developed for a large range of problems. This research investigates two basic techniques (one statistical and one deterministic) which are suitable for the type of information generated in a Monte Carlo calculation. These techniques are used with as little modification as possible from the basic algorithms found in the literature $e^{25,16}$. Thus the purpose of this research is not to design an optimum pattern recognition system.

The Monte Carlo problems used in this research have been chosen so as to minimize computer time while still being useful models for demonstrating the operations of the pattern recognition system. Since many Monte Carlo runs are necessary in research of this type (600 to 700 runs were performed), the computer time would be prohibitive (at least 10 
times as great) using a general purpose code with complex problems.

The majority of parameter tests and classifier evaluation experiments were performed using a one-dimensional, one-region, homogeneous slab Monte Carlo problem. Although such a model is simplified, it still exhibits the characteristics necessary for the application of pattern recognition. Computational experiments are also performed using a onedimensional multi-region $\mathrm{s} l \mathrm{ab}$ and a two-dimensional (distance and direction) multi-region slab. It is found that the only modification to the pattern recognition system necessary for increasing the dimensionality of the problem is to increase the dimensionality of the various vectors involved.

\section{6) Outline of Dissertation}

It is assumed in this dissertation that the reader is familiar with statistical terminology (i.e., mean, variance, probability distribution, etc.) but is not familiar with either the Monte Carlo method or pattern recognition theory. The next two chapters are intend ad to introduce the reader to these topics.

Chapter II introduces the basic principles of Monte Carlo and how statistical errors are calculated. In addition variance reduction techniques are described and the incentives for state space splitting are presented. Finally, a means for measuring the success of a variance reduction technique is described.

Chapter III discusses the general operations of a pattern recognition system. Particular attention is given to the classification 
techniques that are used in this research. The problem of feature selection is only described as it relates to the Monte Carlo problem. In Chapter IV a pattern recognition system is developed for identifying splitting surfaces and performing various parameter tests (items (1) and (2) of Section 1.4). In this chapter a scheme is presented for learning a single splitting surface and is implemented on a one-dimensional one-region slab, a one-dimensional multi-region slab, and a two-dimensional slab Monte Carlo problem. Several parameter tests are made in this chapter and comparisons are made between the different classifiers used. The computer programs used in Chapter IV are given in the Appendices. Although these programs are not implemented on a general purpose Monte Carlo code, several of the timing parameters involved are approxinated.

Chapter $V$ considers the practical problem of implementing the technique for full scale applications. A system suitable for general applications is designed and required user input is noted. Finally, an analysis is performed to determine the effectiveness of the technique. Thus Chapter V treats items (3) and (4) of Section 1.4 . Chapter VI states the conclusions of this research and makes recommendations for implementing this research on a full scale in a general purpose Monte Carlo code. 
II. The Monte Carlo Method and Variance Reduction

Although the Monte Carlo method is applied to a wide range of problems, the emphasis of this research will be on particle transport calculations. It is the purpose of this chapter to describe in very general terms how Monte Carlo sampling is performed and how statistical errors are determined (Section 2.1). For further details of the sampling used for neutron transport, the reader is referred to Appendix A. Section 2.2 contains a description of variance reduction in general and Section 2.3 surveys some of the more common variance reduction techniques including those which involve learning. A method is then proposed which requires learning during the Monte Carlo calculation in order to reduce the variance (St:tion 2.4). Finally, in Section 2.5 effectiveness of Monte Carlo calculations will be defined so that the efrect of variance reduction can be measured.

2.1) The Monte Carlo Method for Particle Transport

\subsection{1) Basic Principles}

The transport problem in this research consists of estimating the probability that particles leaving a source and undergoing various processes (capture, escape, etc.) will finally terminate in a specified category or tally. Decisions as to which processes occur are made by sampling the appropriate "probability distribution" functions as described below. 
The probability that a variable $s$ lies between $s$ and $s+d s$ is given by $\mathrm{p}(\mathrm{s}) \mathrm{ds}$ i:ere $\mathrm{p}(\mathrm{s})$ is defined as the probability density function 1 ? An example of such a function is shown in Figure 2.1 for $s$ ranging from 0 to 3. In this research it is always assumed that $p(s)$ has been nortalized so that

$$
\int \mathrm{p}(\mathrm{s}) \mathrm{ds}=1
$$

where the integral is over all possibles.

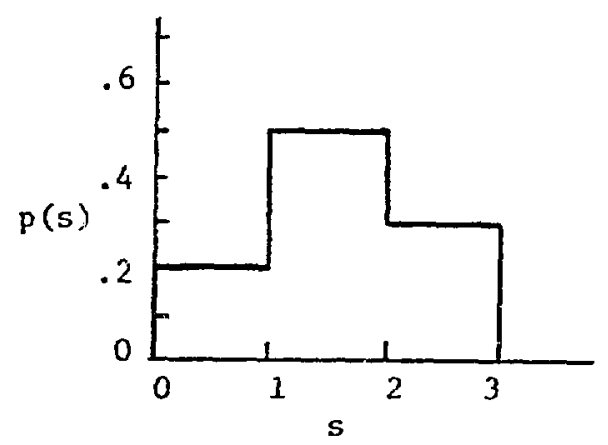

Figure 2.1 A Probability Density Function

The integral of $p(s)$ is defined as the probability distribution function: 


$$
P(s)=\int_{u}^{s} p\left(s^{1}\right) d s^{\prime}
$$

The distribution function of the example in Figure 2.1 is shown in Figure 2.2.

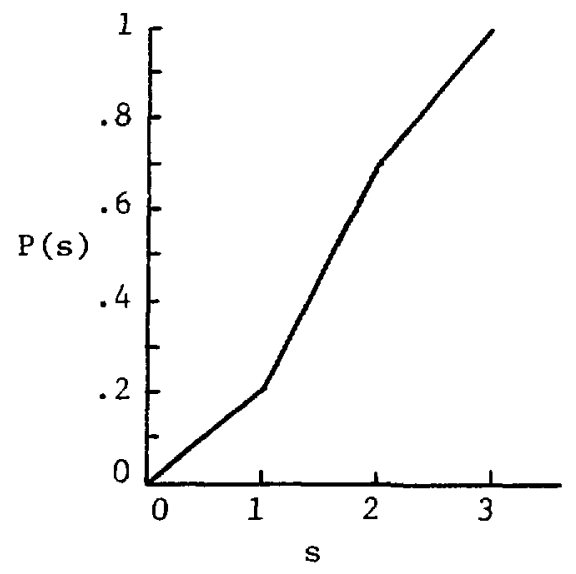

Figure 2.2 A Probability Distribution Function

The probability distribution function is sampled by choosing a random number, $r$, between 0 and $I$ and setting $r$ equal to $P(s)$ as given by

$$
r=\int_{0}^{s} p\left(s^{\prime}\right) d s^{\prime}
$$


The s that satisfies this equality is used as the gabmplat yalue. The yalues of 5 sanpled ia this ramer can be shom to have the probability density $p(s)$ i

As an example of this sempling proccss, cunsider the caste of at neutron eritted from one sice of a slab (sec Figure 2.3) it the ts fircstion.

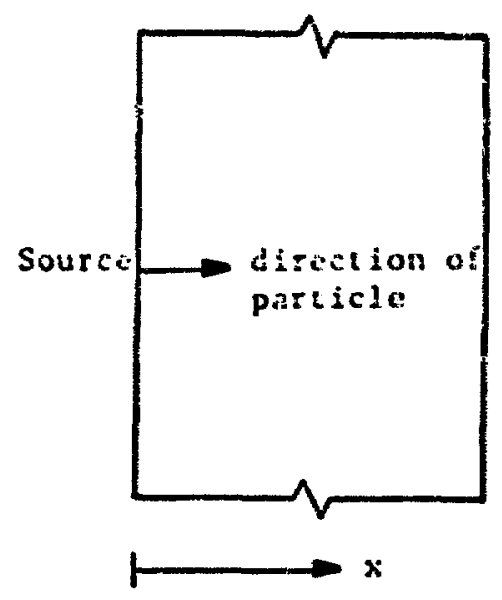

Figure 2.3 Sampling Distance to Collision

The probability that this neutron has a collision between $x$ and $x+d x$ is given by 26

$$
P(x) d x=\varepsilon_{t} e^{-\varepsilon_{t} x} d x
$$

where $\Sigma_{t}=$ the total nacroscopic cross section

$$
\begin{aligned}
P(x)= & \text { the probability density function for a collision } \\
& \text { at } x \text {. }
\end{aligned}
$$


The probabllicy distribution function for this process 13 siven by

$$
\begin{aligned}
& p(x)=\int_{0}^{x} p\left(x^{\prime}\right) d x^{\prime} \\
& \int_{0}^{x} e_{t}^{x^{2}} e^{-3} t^{x} d x \\
& P(x)=1-e^{-2} t^{x}
\end{aligned}
$$

Note that if $x=0, p(x)-1$ and if $x=0, p(x)=0$. Setting a random number, $r$, $(0 \leq r \leq 1)$ equal to the probability distribution function gives

$$
\begin{gathered}
F(x)=1-e^{-E_{c} x} \\
x=\frac{\ln (c)}{E_{t}}
\end{gathered}
$$

where (1-r) has been replaced by $r$. 
The value of $x$ for a given $r$ is the sampled value for the distance to a coilision. In Monte Carlo transport calculations many processes are sampled similar to the example above. Appendix A describes the sampling process in more detail for the case of neutron transport. Eventually, after undergoing numerous events as determined by the appropriate probability distributions, a particle is lost to the system. This occurs when the particle is either captured, leaves the system being considered, falls below the energy range of interest, etc. At this point the contribution, $x_{i}$, of the $i^{\prime}$ th particle to the tally under study is calculated. Thus, for $N$ particles the average contribution to the tally is

$$
\bar{x}=\frac{\sum_{i=1}^{N} x_{i}}{N}
$$

This $\bar{x}$ is the statistical approximation used to estimate the physical quantity of interest. 
The mean value of $N$ samples, $\bar{x}$, is given by Equation 2.3. The variance ${ }^{7}$ of the $\mathrm{N}$ samples with respect to the sample mean $\bar{x}$ is given by

$$
\begin{aligned}
& \bar{\sigma}^{2}(\bar{x})=\frac{1}{N-1}\left[\sum_{i=1}^{N}\left(x_{i}-\bar{x}\right)^{2}\right] \\
& \vec{\sigma}^{2}(\bar{x})=\frac{N}{N-1}\left[\overline{x^{2}}-\bar{x}^{2}\right]
\end{aligned}
$$

The true mean ${ }^{17}$ of $x$ is given by

$$
\langle x\rangle=\int_{-\infty}^{\infty} x(s) p(s) d s
$$

where $\mathrm{p}(\mathrm{s})$ is the probability density function of $\mathrm{s}$. The mean $\langle\mathrm{x}\rangle$ is often referred to as the "expected" ${ }^{1}$ ?alue of $x(s)$. For an unbiased" 7 estimate $\bar{x}$,

$$
\langle\bar{x}\rangle=\langle x\rangle
$$


The true variance of $x(s)$ is difined as the second moment of $x(s)$ about $\langle x\rangle$ as given by

$$
\sigma^{2}(x)=\int_{-\infty}^{\infty}(x(s)-\langle x\rangle)^{2} p(s ; d s
$$

It can be shown (see Appendix is) that the variance of the samples $x_{i}$ about the true mean $\langle x\rangle$ is given by

$$
\sigma^{2}(x)=\frac{\sigma^{2}(x)}{N}
$$

Since in practice neither $\langle x\rangle$ or $\sigma^{2}(x)$ is known, they are approximated by $\bar{x}$ and $\bar{\sigma}^{2}(\bar{x})^{2}$. Making these substicutions and assuming large $N$ results in

$$
\sigma^{2}(\bar{x}) \approx \frac{\bar{\sigma}^{2}(\bar{x})}{N} \approx \frac{\overline{x^{2}}-\overline{x^{2}}}{N}
$$


The Central Limit Theorem (see Appendix B) states that

$$
\operatorname{Prob}|a \bar{\sigma}(x)<(\bar{x}-\langle x\rangle)<\beta \bar{\sigma}(x)| \approx \frac{1}{\sqrt{2 \pi}} \int_{\alpha}^{\beta} e^{-t^{2} / 2} d t .
$$

For $a=-1$ and $B=1$, Equation 2.10 means that there is a $68.3 \%$ probability that the estimated mean is within $\pm \vec{\sigma}(x)$ of the true mean.

Frequently in Monte Carlo calculations it is helpful to express the error in terms of relative error ${ }^{19}$ as given by

$$
\operatorname{Re}=\frac{\sigma(\bar{x})}{\bar{x}} \approx \frac{1}{\sqrt{W}} \frac{\bar{\sigma}(\bar{x})}{\bar{x}}
$$

Equation 2.11 exhibits a very important characteristic of Monte Carlo calculations-- that the error of the sample mean varies as $1 / \sqrt{\mathrm{N}}$. 


\section{2) Variance Reduction}

In order to decrease the error of a Monte Carlo calculation (see Equation 2.11), one must either increase $N$ or decrease $\bar{\sigma}(\bar{x})$. The effectiveness of increasing $N$ to reduce the error is illustrated in Figure 2.4 . From this figure it is apparent that as $N$ increases, the decrease in Re, $\Delta R e$, for a given increase in $N, \Delta N$, decreases. For example, increasing the number of particles from $N=100$ to $N=10,000$ reduces the error by a factor of 10; however, increasing $N$ from 10,000 to 20,000 reduces the error by a factor of only $\sqrt{2}$. Although computer time spent per particle history is an extremely problem dependent parameter, In many cases running time becomes prohibitive after a sample of 10,000 to 100,000 particles. If the relative error is still unacceptably large after several 10,000 particles, additional histories are far too costly for the small amount of error reduction gained.

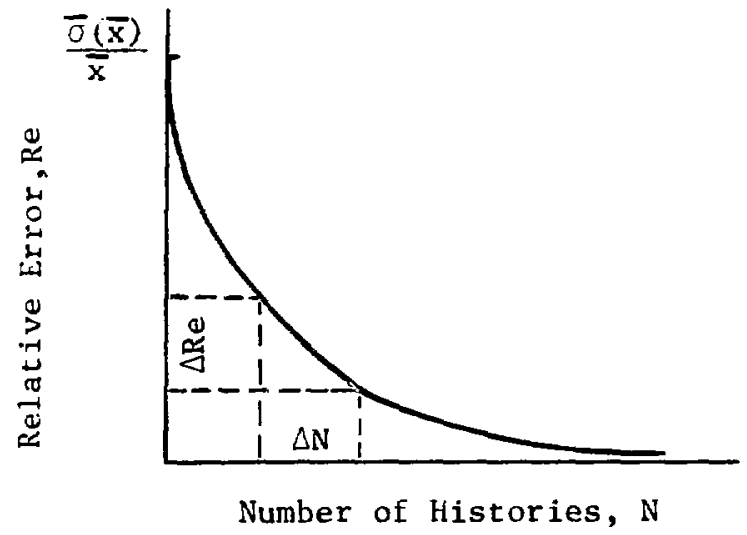

Figure 2.4 Reducing Error by Increasing the Number of Histories 
Bocause of this problem variance reduction techniques are often required to reduce the variance of the sample mean. As seen by Equation $2.4 a$, the only way to do this is to sample values of $x_{i}$ which are closer to $\bar{x}$.

One technique frequently used for doing this is the implementation of particle "weights"7,11 with the elimination of capture. The weight of a particle can be thought oi as representing a fraction of a particle. For cxample, a weight of 1.0 represents an entire particle whereas a weight of 0.5 represents only half a particle. When a particle undergoes a reaction it is never "killed" by a capture, but instead its weight is multiplied by the factor $\Sigma_{n a} / \Sigma_{t}\left(\Sigma_{\text {ra }}=\right.$ non-absorption cross section, $\Sigma_{t}=$ total cross section) and the particle history is continued with reduced weight.

Example. Consider a Monte Carlo problem in which 100 neutrons are started from a source. Of these neutrons $30 \%$ leak out of the system without a collision and $30 \%$ are captured at their first collision. The remaining neutrons undergo one scattering collision after which they are tallied with a value of $x_{i}=1$. Using no weights and assuming neutrons behave exactly as the above percentages indicate, one arrives at:

$$
\bar{x}=\frac{\sum_{i=1}^{100} x_{i}}{100}=\frac{40 \times 1.0+60 \times 0.0}{100}=.4
$$

$$
\bar{\sigma}^{2}(\bar{x})=\frac{1}{100} \sum_{i=1}^{100}\left(x_{i}-\bar{x}\right)^{2}=\frac{1}{100}\left[60 x(.4)^{2}+40 x(.6)^{2}\right]=.24
$$


Using veights and assuming that each neutron that does not escepe widergoes one collision and is then tailied results in:

$$
\bar{x}=\frac{\sum_{i=1}^{100} x_{i} w_{i}}{100}=\frac{30 \times 0.0+70 \times 4 / 7}{100}=.4
$$$$
\bar{\sigma}^{2} \overline{(x)}=\frac{1}{100} \sum_{i=1}^{100}\left(x_{i}-\bar{x}\right)^{2}=\frac{1}{100}\left[30 \times(.4)^{2}+70 \times(.17)^{2}\right]=.0686
$$

A neme applied to a fanily of variance reduction techniques is "importance sampling"1t,il. In transport problems, importance sampling refers to preferentially sampling those particles which are more likely to contribute to the tally being investigated. From a probability density function $p(x)$ the sean value of a function $f(x)$ is given by

$$
\langle E(x)\rangle=\int f(x) p(x) d x
$$

In i=portance sampling an alternate distribution $p_{A}(x)$ is used and the function $f(x)$ is multiplied by $x(x)$ where 


$$
w(x)=\frac{p(x)}{p_{A}(x)} \text {. }
$$

Using this alternate distribution gives a mean of

$$
\left\langle f_{A}(x)\right\rangle=\int f_{A}(x) p_{A}(x) d x=\int f(x)\left[\frac{p(x)}{p_{A}(x)}\right] p_{A}(x) d x=\langle f(x)\rangle
$$

Thus the mean is unchanged. However, the second moment of $f_{A}(x)$ is given by

$$
\begin{aligned}
&\left\langle f_{A}^{2}(x)\right\rangle= \int f_{A}^{2}(x) p_{A}(x) d x=\int\left[\frac{p(x)}{p_{A}(x)}\right] f^{2}(x) p(x) d x \\
&= \int I(x) f^{2}(x) p(x) d x \\
& \text { where } I(x)=\left[\frac{p(x)}{p_{A}(x)}\right] .
\end{aligned}
$$

This is not the same as the second moment of the unaltered distribution which is 


$$
\left\langle f^{2}(x)\right\rangle=\int f^{2}(x) p(x) d x
$$

In Equation $2.15<f_{A}^{2}(x)>$ can be reduced by decreasing $I(x)$ where $p(x)$ is large. This requires that $I(x)$ be increased where $p(x)$ is small. In the ideal case $P_{A}(x)=\frac{f(x) p(x)}{\langle f(x)\rangle}$ resulting in

$$
\begin{aligned}
& \sigma^{2}\left(f_{A}(x)\right)=\left\langle f_{A}^{2}(x)\right\rangle-\langle f(x)\rangle^{2} \\
= & \int\left[f(x) \frac{p(x)}{P_{A}(x)}-\langle f(x)\rangle\right]_{A}^{2}(x) d x=0
\end{aligned}
$$

However, before this zero variance $p_{A}(x)$ can be found, the mean $\langle f(x)\rangle$ must be known, which of course is never the case. In the following section several techniques based on Inportance sampling will be discussed.

\section{3) Survey of Variance Reduction Techniques}

During the development of Monte Carlo, there have been numerous techniques proposed to reduce the variance of the Monte Carlo tally. However, when one looks at the major Monte Carlo codes, he finds that only very few of these techniques are used. One of the reasons for this is that many methods are "unsafe" to use because they may bias the answer or may 
actually consume more computer time than they save. Other methods are rarely used because they are too complex to implement. The majority of this section will be devoted to the description of splitting ${ }^{34}$ and Russian roulette $^{14}$ which are perhaps the most widely used variance reduction techniques. A brief description of some other popular techniques will also be given. In Section 2.3.3 techniques which require "learning" during the calculations will be discussed.

\subsection{1) Splitting and Russian Roulette}

Splitting accompanied by Russian roulette is one of the most commonly used variance reduction techniques. It consists of dividing the geometry of the problem into regions and assigning an importance to each region. This "importance" is selected so that particles in a region of high importance have a higher probability of contributing to the tally. A particle going from a region of low importance to one of greater importance is split at the boundary between the regions into two or more particles (the number depends on the ratio of the import inces) with each new particle having a reduced weight. A particle entering a region of lesser importance is terminated or "killed" with a probability determined by the ratio of importances. If the particle survives the Russian roulette, its weight is increased proportionately.

Figure 2.5 shows an example of splitting planes and importance regions used with an infinite slab of thickness $T$. For problems in which $T$ is many mean free paths, splitting and Russian roulette can be very effective and of ten lead to several orders of magnitude reduction in 


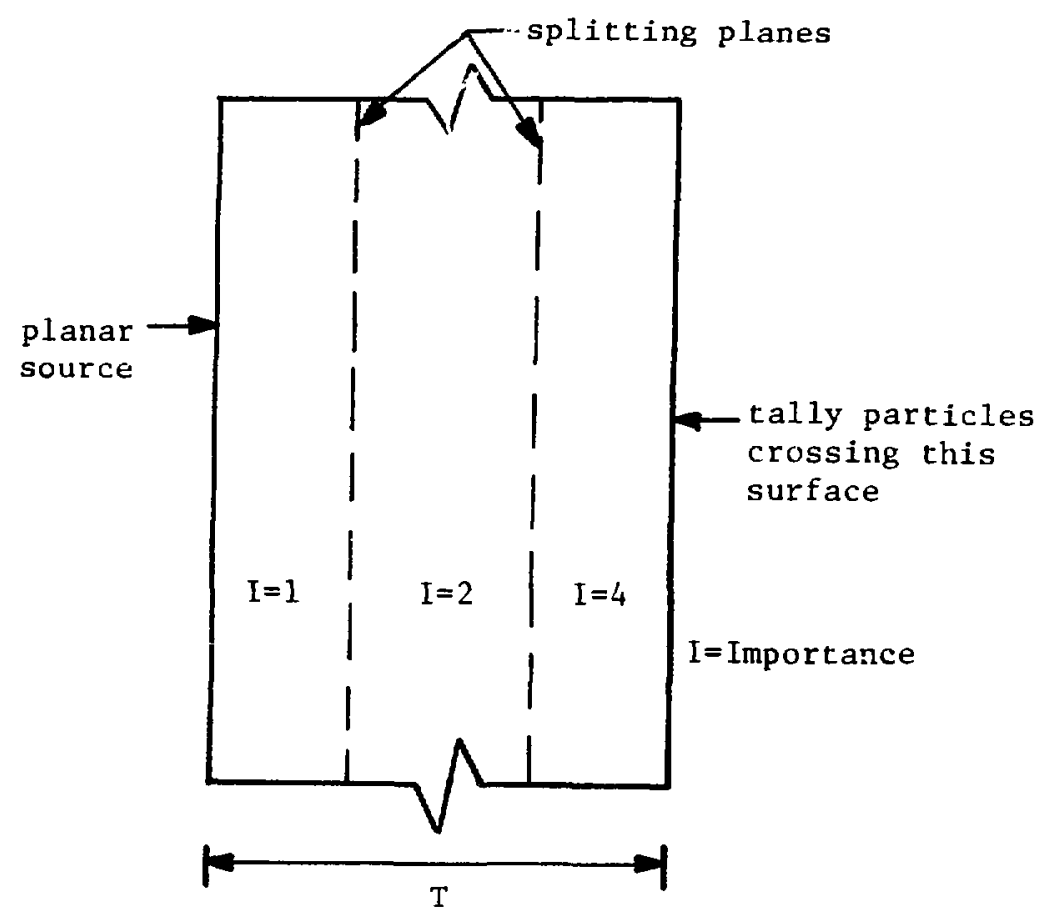

Figure 2.5 Splitting Planes in One Dimension

Splitting and Russian roulette can also be used in energy space for problems in which particular energy regions are more important than others. An example of "energy splitting"l4 is the tallying of $\mathrm{U}^{235}$ thermal fission. In this case, one rould separate energy space into regions which increase in importance as thermal energies are approached as shown in Eigure 2.6. 


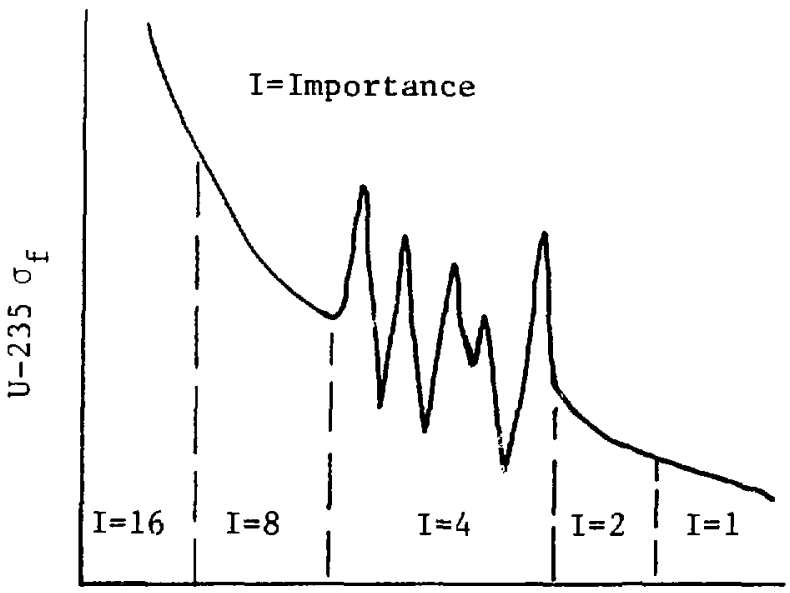

Energy

Figure 2.6 Energy Splitting

The popularity of the above techniques can be attributed primarily to the ease of their implementation. In most cases only a very rough guess based on intuition will lead to a large savings in computer time. Usually the importance regions specified are already geometrically defined by the problem (different materials, densities, and shapes) and the user only has to provide the importances.

\subsection{2) Other Techniques}

A very simple technique commonly used is source biasing ${ }^{7}, 14$. In source biasing important particles are produced more frequently but with reduced weights. An example of source biasing is shown in Figure 2.7. 


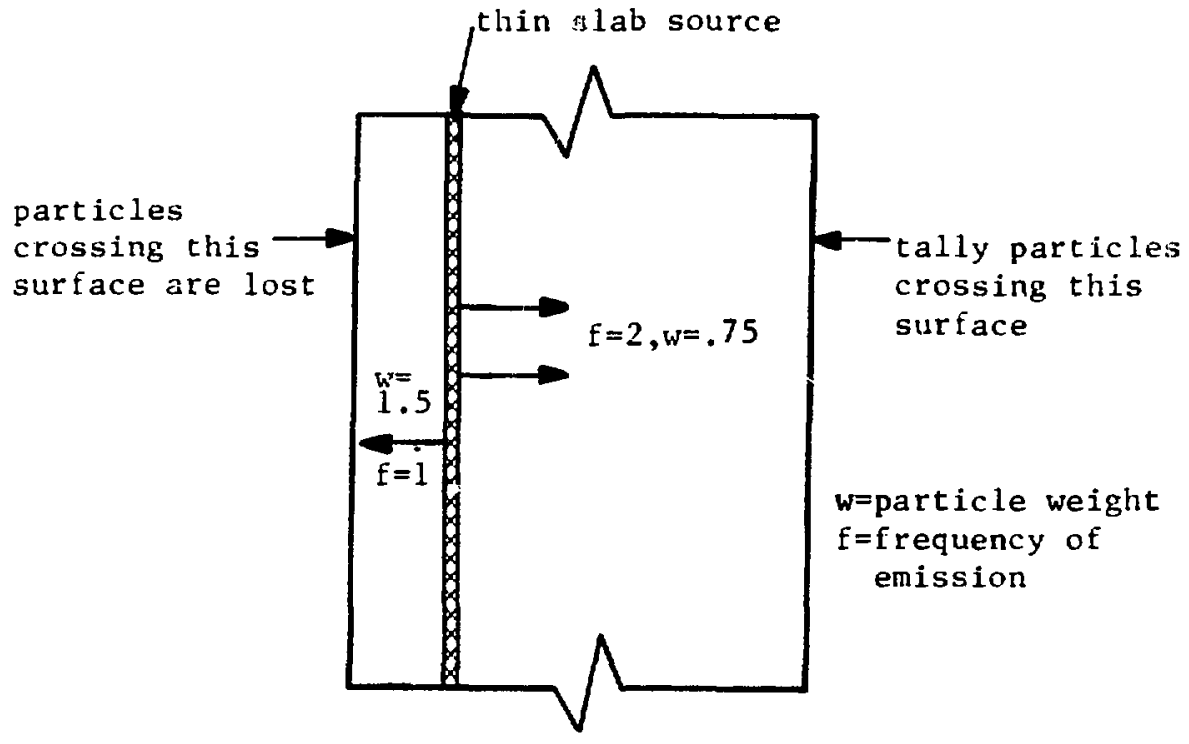

Figure 2.7 Source Biasing

In this example twice as many particles are started to the right as to the left. However particles to the left have twice as much weight. Another method used to increase the number of particles in important regions is the exponential transform ${ }^{11,14,30}$. This technique transforms the transport equation, resulting in the replacement of $\Sigma_{t}$ by $\Sigma_{t}-w$ where $w$ is the direction cosine of the line of flight of the particle with the preferred line of flight. Figure 2.5 shows the case where the desired line of flight is the $x$-axis. The weight of a particle entering a collision is multiplied by 


$$
\frac{\Sigma_{t}}{\Sigma_{t}-\alpha_{w}} e^{-\alpha_{w s}}
$$

where $s$ is the distance traveled before collision.

A problem in using many importance sampling techniques is that of choosing near optimum biasing parameters. This is frequeztly done by rough calculations or maybe even a few preliminary Monte Carlo calculaticns. Another method is to use the solution to the adjoint ${ }^{14,20}$ of the provlem to estimate these parameters. The computation of importance sampling functions has also been automated by other means.

other variance reduction techniques include stratified sampling ${ }^{1}$, antithetic variates ${ }^{11}$, scattering angle biasing ${ }^{21}$, wethod of expected values $^{11}$, correlated sampling ${ }^{11,29}$, and others ${ }^{32}$.

\subsection{3) Variance Reduction Through Learning}

In all of the previously mentioned techniques, importance sampling parameters had to be provided prior to the execution of the Monte Carlo calculations. In this section techniques will be described which allow the variance reduction technique to improve during operation by learning from early histories of the calculations.

Spanier ${ }^{9}$ applies a learning technique to the exponential transform using a one-dimensional slab as an example. The parameter $\alpha$ (see Section 2.3.2) is optimized by making estimates of $\left\langle f_{A}^{2}(x)\right\rangle$ for several values of $\alpha$ while histories are being generated on the basis of the 
parameter value $\hat{\alpha}$. An $\alpha$ which minimizes $\left\langle\mathrm{f}_{A}^{2}(\mathrm{x})\right\rangle$ can then be used in another iteration as the next $\hat{\alpha}$. This process continues until satisfactory agreement is reached between two stages. In the examples given three iterations were sufficient and led to a greatly reduced variance. MacMillan'o suggests a refinement on Spanier's method involving estimates of the first and second derivatives of $\left\langle f_{A}^{2}(x)\right\rangle$ with respect to $\alpha$ and using these estimates to improve the approximation of $\hat{\alpha}$ in going from one iteration to another.

A multistage self-improving Monte Carlo method ${ }^{12}$ has been described which divides space into volumes $V_{i}$ and assigns each volume a weight $p_{i}$ where $p_{i}$ determines the amount of sampling for associated $V_{i}$. The Monte Carlo calculation then proceeds in stages after which $p_{i}$ and $V_{i}$ are altered in such a way as to reduce the variance. This method is analogous to learning the optimum importances for different geometry regions only in this case the extent of the regions is rariable. For small probability problems the range of the tally is enlarged to increase the probability until suitable $v_{i}$ and $p_{i}$ are learned after which the tally is reduced to its original specifications. Running times have been reduced as much as a factor of 100 using this method over the crude Monte $\operatorname{Car} 10^{12}$

The Spanier and MacMillan techniques are primarily concerned with the directional variables of a Monte Carlo problem and since they are based on the exponential transform, they can be unsafe to use. The multistage technique is concerned primarily with spatial variables. Furthermore, this technique has disadvantages when used with Monte Carlo problems with low probability. 
Consider the general Monte Carlo problem in which particles are characterized by the following state variables:

- Spatial coordinates-x,y, and $z$

- Angular coordinates-u,v, and $w$, where these values are the cosines of the particle line of flight with the $\mathrm{x}, \mathrm{y}$, and $\mathrm{z}$ axis respectively.

- Energy-E

- Time-t

In Section 2.3.1 splitting and Russian Roulette were described primarily as applied to the spatial coordinates. Independent application was also mentioned with respect to the energy variable.

Theoretically, it would be quite effective if splitting could be used in the entire state space. In other words all variables would be considered to determine which regions in state space are more important than others. A practical problem arises in determining the importances of these state space regions. Users have trouble enough with the three spatial coordinates; the complexity involved in determining splitting surfaces in eight dimensions would certainly confuse even the most experienced user.

As has been seen in the previous sections, there is a considerable amount of information generated during a Monte Carlo calculation which can be used to accelerate the calculation. However, utilization of this information can become costly in terms of computer time and storage. In this research, pattern recognition techniques are used to 
learn these splitting surfaces during the calculations.

Such a technitye would be an improvement over the learning techniques described in Section 2.3.3 for two reasons:

(1) All state space parameters would be considered, not just directional or spatial quantities.

(2) The technique is based on the splitting and Russian roulette techniques which have proven to be the most popular and useful techniques.

\section{5) Effectiveness of Variance Reduction Techniques}

Although it is certainly useful in Monte Carlo calculations to reduce the variance, the primary goal is to reduce the amount of computer time spent on a calculation. It is quite possible to use variance reduction to decrease $\sigma$ for a given $\mathrm{N}$ but in so doing to increase the time spent per particle to such an extent that it would be cheaper just to run more particles. Therefore, the parameter to minimize is the time required to obtain the desired relative error as given by

$$
t_{c}=N_{e} \Delta t_{e}
$$

$$
\text { where: } \begin{aligned}
\mathrm{N}_{\mathrm{e}}= & \begin{array}{l}
\text { number of histories required to obtain } \\
\text { the desired Re (see Equation 2.11) }
\end{array} \\
\Delta t_{e}= & \text { time spent per neutron history }
\end{aligned}
$$


Variance reduction techniques decrease $s_{e}$ but increase $\Delta t_{e}$. If $t_{c}{ }^{*}, N_{e}^{*}$, and $\Delta t e^{*}$ are the parameters of a calculation without using variance reduction, then the relative effectiveness of a technique can be defined as

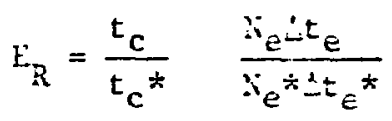

It should be remembered that $E_{R}$ is relative to the following factors:

(1) the cosputing achinery being used,

(2) the efificiency of tine Uonte Carlo calculation without variance reduction,

(3) the characteristics of the particular problem under study and,

(4) the programing efficiency used to implement the technique (i.e., assezbiy language vs. FORTP.si etc.).

Of the above, point three is the rost important since the effectiveness of a technique is strongly dependent on the problem to which it is being applied.

Computer tise spent during a calculation is not the only measure of performance for evaluating variance reduction techniques. Another parameter is the amount of human effort (and sometimes additional computer time) required to implament a technique. The majority of techuiques in use require a certain atount of a priori information. Thus the 
implementation of some techniques is an art in itself requiring considerable ingenuity and experience on the part of the user. In addition some techniques require that calculations be performed in order to determine importance parameters, etc. Figure 2.8 illustrates the operations and times required in implementing a variance reduction technique. The importance attributed to time spent on each of these operations is an extremely subjective function yet it certainly influences the overall acceptability of a technique.

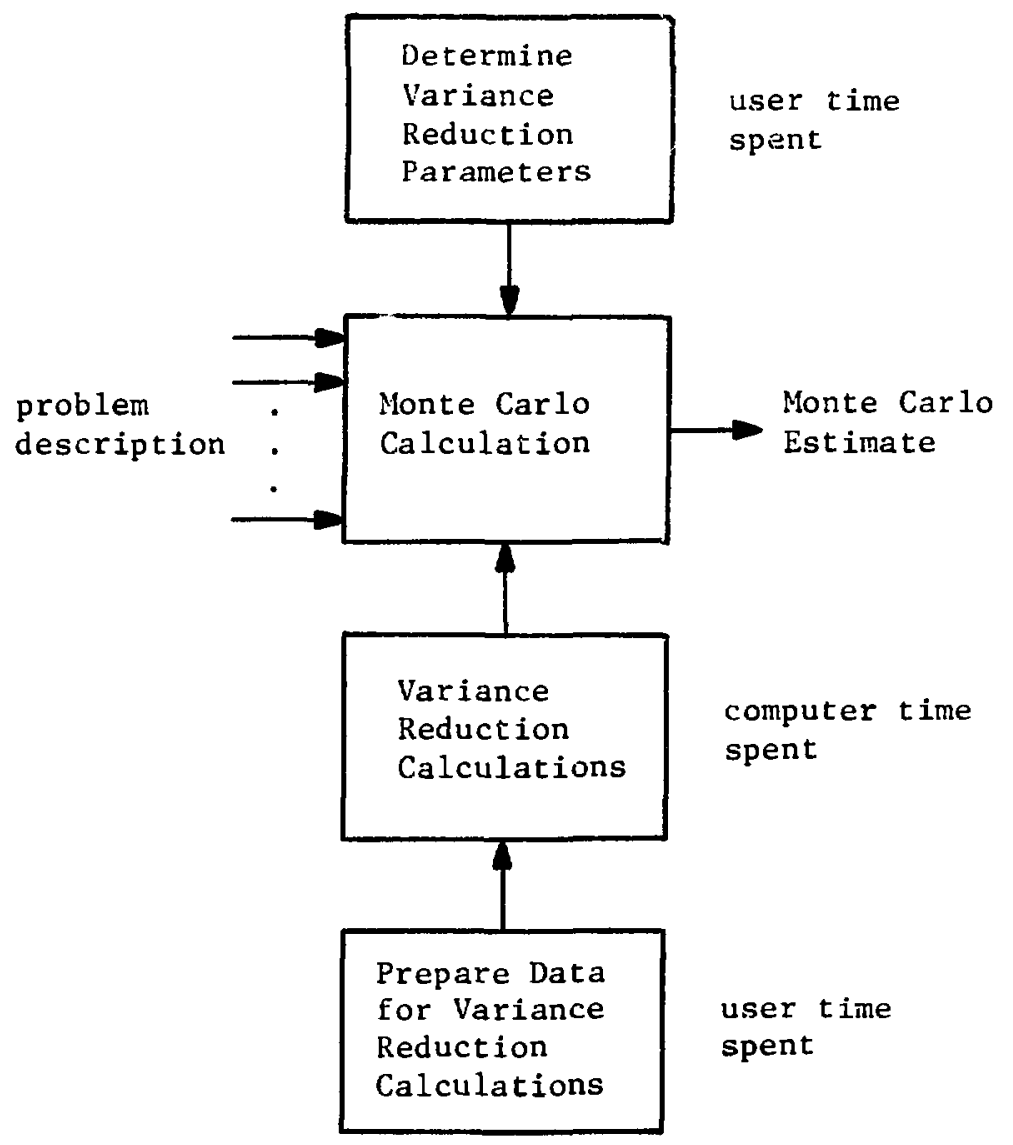

Figure 2.8 Implementation of Variance Reduction Techniques 


\section{Pattern Recognition}

The field of pattern recognition includes an extremeiy broad range of topics including engineering applications, artificial intelligence studies, biological systems, and others. Because of its diffuse application, a general theory of pattern recognition is difficult to separate from its applications. To confuse matters further, it appears that even the introductory texts $15,16,22,23,24,25,26$ on pattern recognition do not agree on a unified framework for describing pattern recognition systems. As a result, a novice in the field frequently encounters a variety of new vocabulary words describing types of pattern recognition systems including such terminology as statistical, parametric, non-parametric, sequential, distribution free, stochastic, nonsupervised, supervised, error-correcting, Bayesian, etc.

The purpose of this chapter is not to explain all facets of pattern recognition to the reader, but only to provide him with the tools necessary to understand how pattern recognition is to be used in this research.

A general pattern recognition system will be explained in Section 3.1 in terms of the basic operations performed. The different types of pattern classification algorithms will then be classified according to the type of input data they require. Sections 3.2 and 3.3 describe in more detail the type of pattern classification algorithms to be used in this research. Section 3.4 discusses the problem of feature selection with emphasis on the Monte Carlo transport problem. Finally, Section 3.5 expands the previous explanations to multiclass problems. 


\section{1) The General Pattern Recognition Problem}

3.1.1) Fundamentals

The primary difficulty with understanding the fundamentals of a pattern recognition system is due to the terminology used. This section introduces pattern recognition terminology by relating the concepts to the simple example of weather prediction as given below:

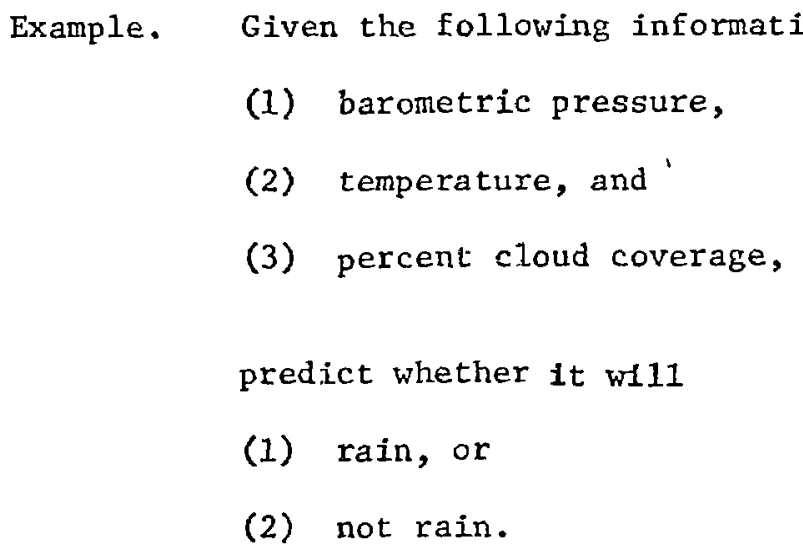

The input - output relationship of a system to perform this task is shown in Figure 3.1 .

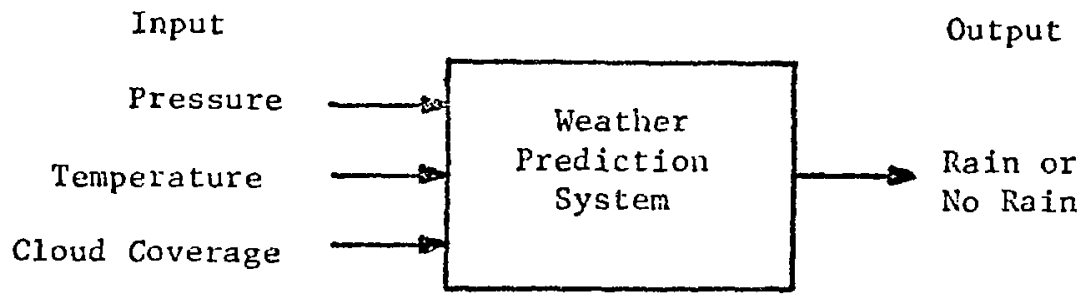

Figure 3.1 Input-Output Model of Weather Prediction 
A coordinate system defined by the input variables of Figure 3.1 is shown in Figure 3.2

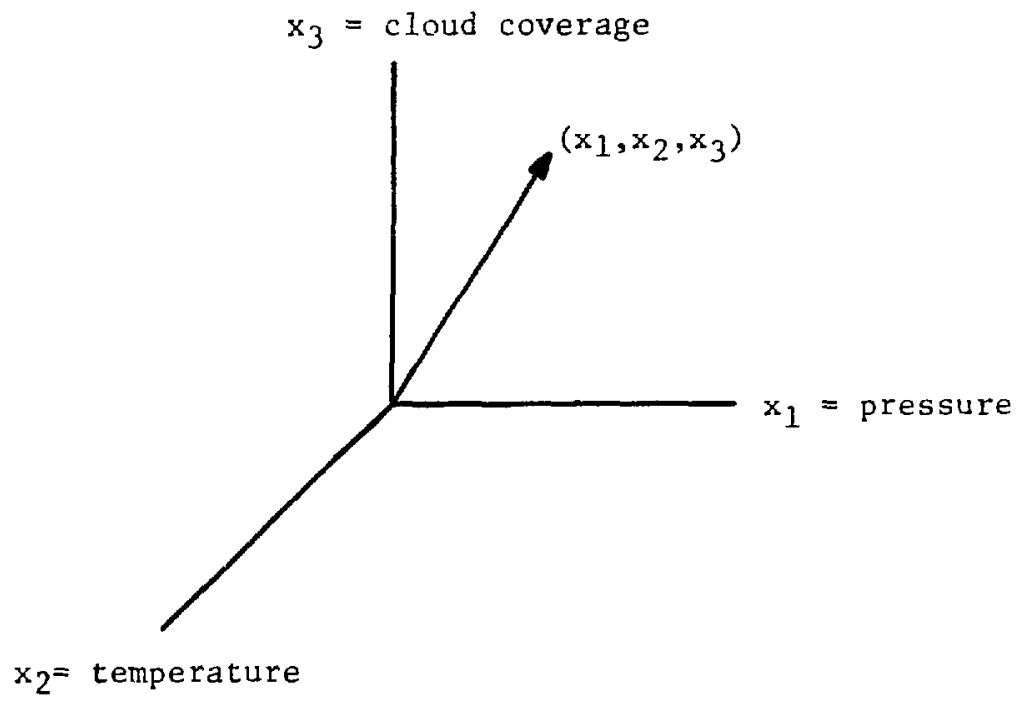

Figure 3.2 Pattern Space

and is referred to as pattern space. The vector drawn from the origin of pattern space to the point $\left(x_{1}, x_{2}, x_{3}\right)$ in Figure 3.2 is called the pattern vector and in this dissertation will be designated by

$$
\mathrm{x}=\left[\begin{array}{c}
\mathrm{x}_{1} \\
\mathrm{x}_{2} \\
\dot{\cdot} \\
\dot{\mathrm{x}_{\mathrm{R}}}
\end{array}\right]
$$


where $R$ is the dimension of pattern space $(R=3$ for the example shown in Figure 3.2). The purpose of the pattern recognition system in this exanple is to divide pattern space into two refions: (1) those $\mathrm{X}$ wich indicate rain and (2) those $X$ which indicate no rain. The options rain and no rain are called classes and are referred to as $C_{1}$ and $C_{2}$ where

$$
\begin{aligned}
& c_{1}=\text { rain and } \\
& c_{2}=\text { no rain. }
\end{aligned}
$$

In general, the purpose of a pattern recognition system is to classify pattern vectors into their appropriate classes $C_{1}, C_{2}, \ldots, C_{K}$ where $K$ is the number of classes (see Figure 3.3).

Pattern Vector, $\mathrm{X} \quad$ Classification

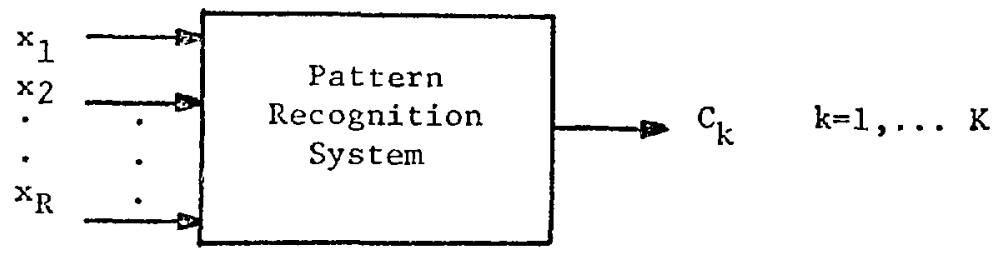

Figure 3.3 Input-Output Model of a Pattern Recognition System

The structure of a pattern recognition system can often be simplified if patten space is transformed into a more efficient configuration. 
For instance, if in the weather prediction example, it is found that the temperature is of no value for predicting rain (i.e. there is no correlation between temperature and rain) and that the probability of rain increases In proportion to the square of the percent cloud cover, then it would be more efficient to use the coordinate system shown in Figure 3.4.

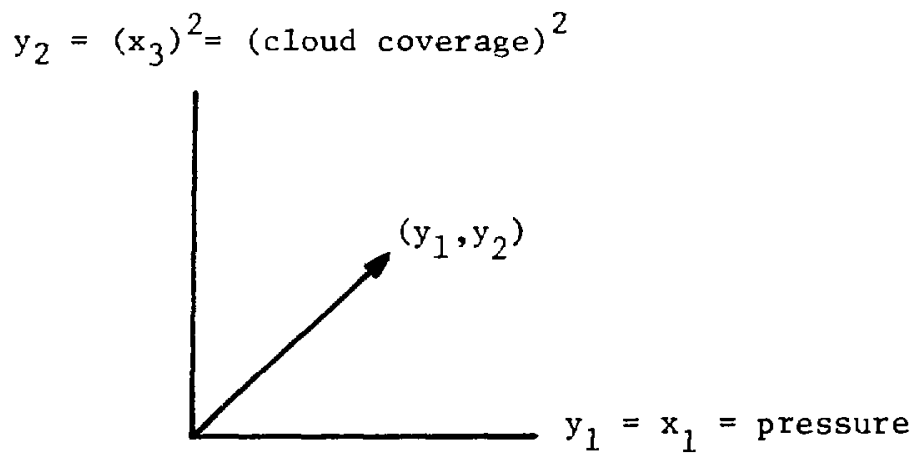

Figure 3.4 Feature Space

This new coordinate system is referred to as feature space. The vector from the origin of feature space to the point $\left(y_{1}, y_{2}\right)$ is called the feature vector and in this dissertation is designated by

$$
\mathrm{Y}=\left[\begin{array}{c}
\mathrm{y}_{1} \\
\mathrm{y}_{2} \\
\dot{\vdots} \\
\mathrm{y}_{\mathrm{N}}
\end{array}\right]
$$


where $X$ is the dimensionality of zeature space. The process of transforning a pattern vector into a feature vector is called feature selection*. The input - oucput relationship of a feature selector is shown in figure 3.5 where in general $\pi<R$.

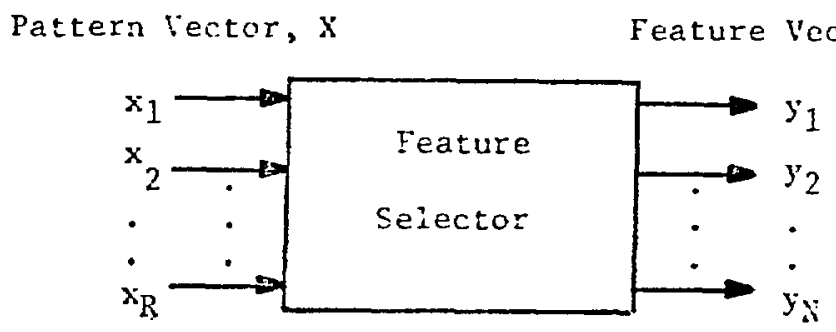

\section{Eigure 3.5 5eature Selection}

The feature selection operation is hishly problem dependent and will be discussed further in Section 3.4 .

The operation of classifying the feature vector into classes $\mathrm{c}_{1}, \mathrm{c}_{2}, \ldots, \mathrm{c}_{\mathrm{K}}$ is called pattern classification. Thus the pattern recognition system consists of two najor components: feature selection and pattern classification (see Figure 3.6).

* In this dissertation any cperation performed on the pattern vector prior to classification is considered to be a feature selection operation. 


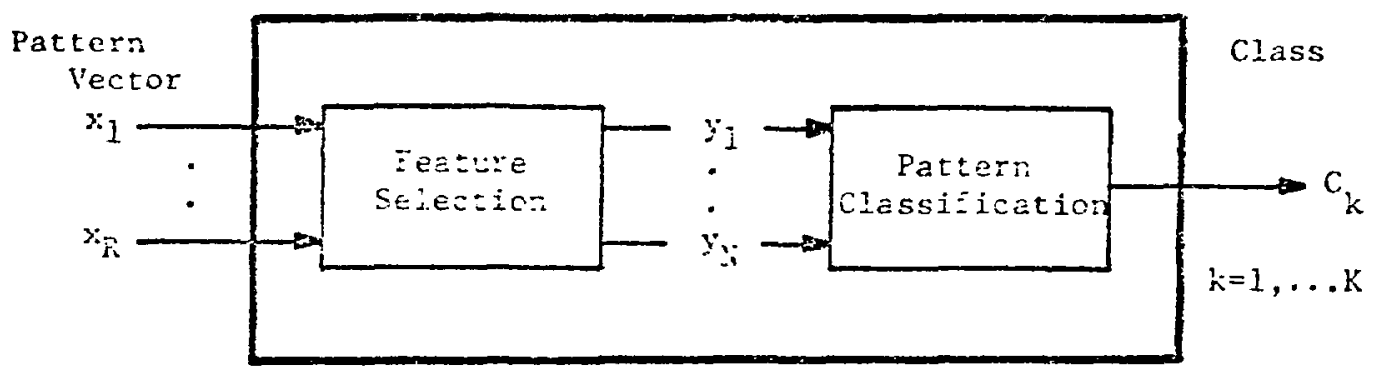

Figure 3.6 A Eattern Recognition System

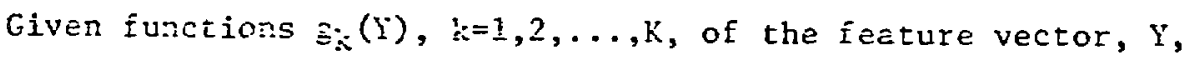
such tiret

$$
\text { if } g_{g}(I)>g_{i}(Y) \quad i=1,2, \ldots, k \quad i \neq k
$$

then $Y$ is placed in class $C_{k}$ by the pattern classifier. The function $g_{k}(Y)$ is called the discriminant function of $C_{k}$. If $K=2$, as in the weather forecasting example, a discri-:inant function $g_{1,2}\left({ }^{*}\right)$ can be defined such that 


$$
g_{1,2}(Y)=g_{2}(Y)-g_{1}(Y)
$$

In this case,

$$
\begin{aligned}
& \text { if } g_{1,2}(Y)>0 \text {, then } Y \text { belongs to } C_{2} \\
& \text { if } g_{1,2}(Y)<0 \text {, then } Y \text { belongs to } C_{1} \text {. }
\end{aligned}
$$

The surface for which

$$
\begin{aligned}
g_{k}(Y)=g_{i}(Y) \quad & k=1,2, \ldots . k \\
& i=I, 2, \ldots . k \\
& i \neq k
\end{aligned}
$$

is called the decision surface between $c_{k}$ and $c_{i}$. The decision surfaces separate feature space into $\mathrm{K}$ regions. The $\mathrm{Y}$ 's in each region belong to the same ciass. For a two class problem the decision surface is given by

$$
g_{1,2}(Y)=0
$$


Such a surface (a line in this case) is shown in Figure 3.7 for the weather forecasting example.

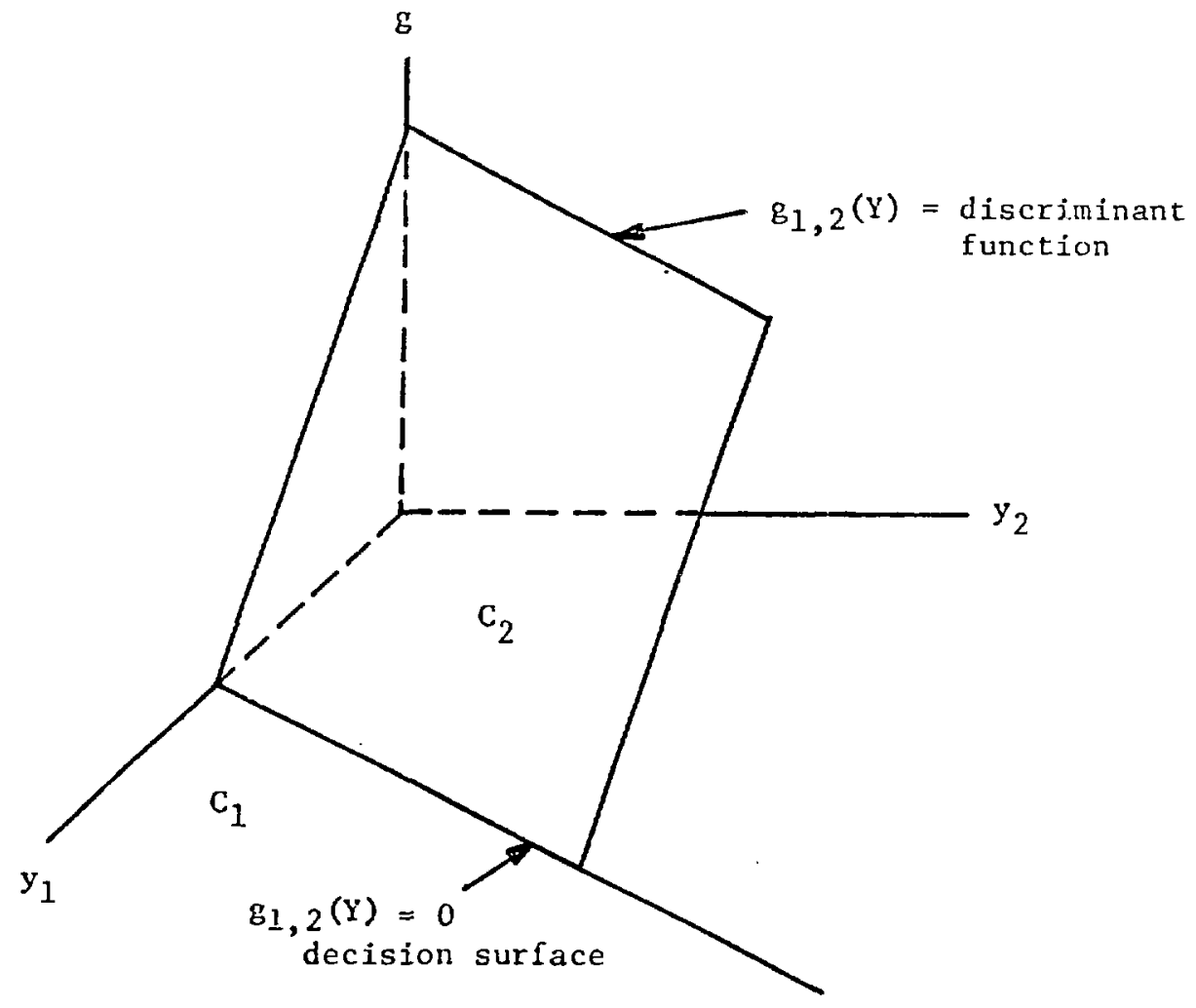

Figure 3.7 Decision Surface

The previous discussion is concerned with how features are classified and is true for pattern classifiers in general. However, before the classifier can operate, the form of the discriminant functions, $\varepsilon_{k}(Y)$, must be known. How the $g_{k}(Y)$ are arrived 
at for different pattern classifiers is the subject of the next section.

\subsection{2) Types of Pattern Classifiers}

Every feature vector has associated with it a probability of belonging to a given class. This probability will be denoted by $p\left(C_{i} \mid Y\right)$ which is the probability that feature vector $Y$ belongs to class $C_{i}$. For a two-class problem, if one class can be uniquely associated with each pattern such that

$$
\begin{aligned}
& \text { if } \mathrm{p}\left(\mathrm{C}_{1} \mid \mathrm{Y}\right)>0 \text { then } \mathrm{p}\left(\mathrm{C}_{2} \mid \mathrm{Y}\right)=0 \\
& \text { if } \mathrm{p}\left(\mathrm{C}_{2} \mid \mathrm{Y}\right)>0 \text { then } \mathrm{p}\left(\mathrm{C}_{1} \mid \mathrm{Y}\right)=0 \text {, }
\end{aligned}
$$

then the classes are said to be non-overlapping. If patterns can belong to one class sometimes and the other class at other times, the classes are said to be overlapping. Examples of these two different types of distributions are shown in Figure 3.8 using the weather prediction example. 


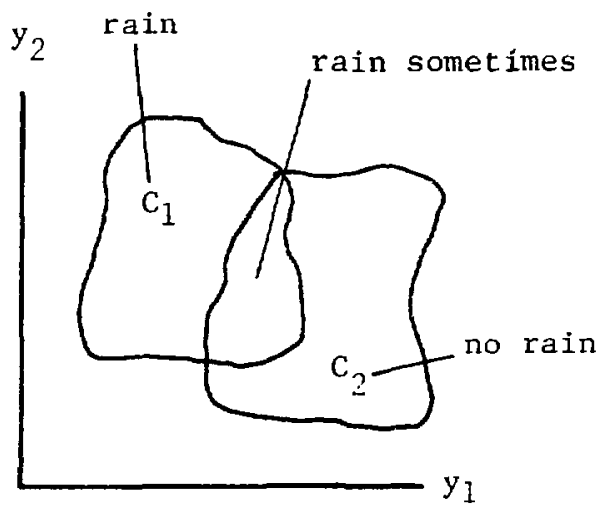

(a) Overlapping

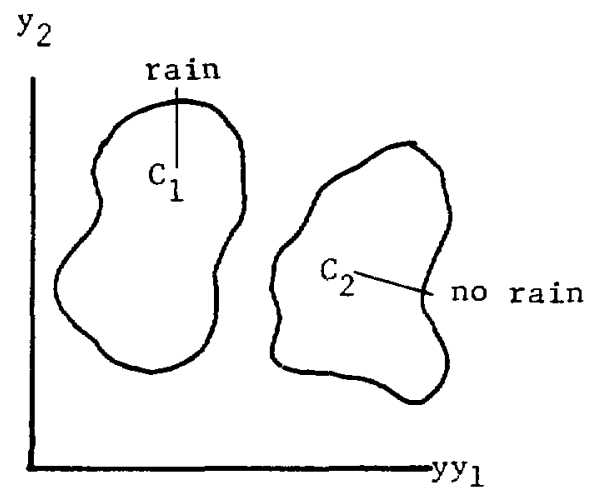

(b) Non-Overlapping

Figure 3.8 Overlapping and Non-Overlapping Classes

Classifier input data and the schemes they require can be separated into different cases depending on what is known about $p\left(C_{i} \mid Y\right)$ and the input data ${ }^{27}$. There are basically four types of information of which one or more may be available to the classifier. These information types $\operatorname{are}^{27}$ :

(1) Functional form of $p\left(C_{i} \mid Y\right)$ is known. For example it may be known that both $\mathrm{p}\left(\mathrm{C}_{1} \mid \mathrm{Y}\right)$ and $\mathrm{p}\left(\mathrm{C}_{2} \mid \mathrm{Y}\right)$ are Gaussian but with unknown means and variances.

(2) Parameters of $p\left(C_{i} \mid Y\right)$ are known. Parameters include the mean, variance, etc.

(3) Sample pattern vectors with known classification are given. Each pattern vector with its classification is called a prototype. These prototypes serve as a training set for the classifier. 
(4) Sample pattern vectors of unknown classification are given.

Depending on which of the above information is available, six major kinds of pattern classifiers can be defined ${ }^{27}$.

(1) Case A: Information types 1 and 2 are given

(2.) Case B: Information types 1 and 3 are given

(3) Case C: Information types 1 and 4 are given

(4) Case D: Information type 3 is given, Deterministic methods are used

(5) Case E: Informaticn type 3 is given, Statistical methods are used

(6) Case F: Infornation type 4 is given

In Case $\mathrm{A}$ a.11 the in formation required to make an analytical solution for $g(Y)$ is known. In Case $B$ the classified $Y$ 's must be used to make an approximation of the required parameters after which the classifier becomes a Case A. Cases $\mathrm{C}$ and $\mathrm{F}$ are often referred to as "Iearning without a teacher" or unsupervised learning ${ }^{16}$ and usually consist of a type of clustering technique. ${ }^{26}$

In Case $D$ the basic iciea is to find a $g(Y)$ which operates "satisfactorily" on the samples of known classification. This type of approach, sometimes referred to as "distribution free" cerning the $\mathrm{p}\left(\mathrm{C}_{\mathrm{i}} \mid \mathrm{Y}\right)$. Instead the data is assumed to be separable by a given form of $\mathrm{g}(\mathrm{Y})$, i.e., linear, quadratic, etc. One drawback of such an approach is that it places an additional burden on the feature selector in order to produce feature vectors which satisfy the assumptions made on $\mathrm{g}(\mathrm{Y})$. 
Case $E$ consists of using statistical techniques tc minimize classification errors. These techniques are theoretically more useful for overlapping data since they allow for the existence of error. Frequently in the statistical approach $p\left(C_{i} \mid Y\right)$ is expanded in a series ${ }^{26}$

$$
p\left(c_{i} \mid Y\right)=\sum_{j=1}^{J} \alpha_{i j} \phi_{j}(Y), \quad i=1,2
$$

where the $\alpha_{i j}$ 's are approximated by using prototypes. A simpler approach is similar to the deterministic approach and consists of assuming a form for $g(Y)^{2} \cdot 8$ However, unlike the deterministic classifier, the input data need not conform to the assumptions made on $g(Y)$ since in this case $g(Y)$ is approximated by the statistical behavior of the data in order to minimize the number of misclassifications.

Classification techniques for Cases B through F can be further characterized as sequential $1^{23}$ or non-sequential techniques ${ }^{36}$. In sequential techniques the prototypes are presented one at a time and approximations are made concerning $g(Y)$ or $p\left(C_{i} \mid Y\right)$ as each prototype is presented. In non-sequential techniques a finite number of prototypes is presented at once to the classifier and an optimum $g(Y)$ or $p\left(C_{i} \mid Y\right)$ is fitted to these prototypes.

In summary, the selection of a pattern classification scheme depends upon the information available (Cases A-F), whether the $p\left(C_{i} \mid Y\right)$ are overlapping, and the manner in which the prototypes are presenied (sequential or non-sequential). Because of the characieristics 
of Monte Carlo problems, only Case D and E classifiers will be investigated in this study. A more detailed description of these classifiers using sequential learning methods is presented in Sections 3.2 and 3.3 .

\section{2) Sequential Deterministic Classification Techniques (Case D)}

In this section several deterministic techniques are described for classifying patterns when prototypes of known classification are presented sequentially. This approach consists of assuming a form for $g(Y)$ and using the prototypes to learn the necessary parameters. Two class problems $(K=2)$ are assumed resulting in a single discriminant function $g(Y) \equiv g_{1,2}(Y)$ (see Equation 3.2).

\subsection{1) Linear Discriminant Functions}

The general form of a linear discriminant function for $\mathrm{N}$ dimensional feature space is given by

$$
\begin{gathered}
\mathrm{g}(\mathrm{Y})=\mathrm{w}_{1} \mathrm{y}_{1}+\mathrm{w}_{2 \mathrm{y}_{2}}+\ldots \ldots \mathrm{w}_{\mathrm{N}} \mathrm{y}_{\mathrm{N}}+\mathrm{w}_{\mathrm{N}+1}=\mathrm{w}^{\mathrm{T} \mathrm{Y}^{*}} \\
\text { where } \quad \mathrm{Y}^{*}=\left[\begin{array}{c}
\mathrm{y}_{1} \\
\mathrm{y}_{2} \\
\cdot \\
\mathrm{y}_{\mathrm{N}} \\
1
\end{array}\right], \quad \mathrm{W}=\left[\begin{array}{l}
\mathrm{w}_{1} \\
2 \\
\cdot \\
\cdot \\
\mathrm{w}_{\mathrm{N}+1}
\end{array}\right] \\
\mathrm{W}^{\mathrm{T}}=\text { the transpose of } \mathrm{W}
\end{gathered}
$$


and the vector $\because$ (the weigis vector) wust be obtained from information contained in the prototypes. The vector $\mathrm{Y}^{*}$ is called the augmented ${ }^{16}$ feature vector and is of cimension $\mathrm{i}+\mathrm{l}$. The use of $\mathrm{g}(\mathrm{I})$ as given by Equation 3.8 assures that feature space is linearly separable. For twodinensional fectue space this zeans that all feature vectors belonging to $C_{1}$ can be separated by a linear decision surface (a straight line for $:=2$ ) from all feature vectors belonging to $C_{2}$. Figure 3.9 illustrates linearly and mon-1ineariy separable feature vectors. Note that for the linearly separable data show (Eigure 3.9a), there is an infinite number of decision surfaces with satisfactorily seacrate feature space. For the data of Figure 3.9b there is no linear $\xi\left(I^{*}\right)=0$ that will separate the classes. The cooridiate systen created $y$ the components of the weight vactor, W (see Eçuation 3.8), is rejerred to as weight space and is frequently used to explain the behavior of deterinistic classifiers. height spaca for two-dirensional feature space is shown in Figure 3.10, where the vector from the origin to the poirt $\left(w_{1}, w_{2}, w_{3}\right)$ is called the weight vector. It should be noted that wight space is of dimension $14+1$ when feature space is of dimension $X$.

Consider the one-dinensional feature space shown in Figure 3.11 where

$$
\begin{aligned}
& \text { if }-5 \leq y \leq 3 \quad y \text { belongs to } C_{1} \\
& \text { if } 10 \leq y \leq 15 \quad y \text { belongs to } C_{2} \text {. }
\end{aligned}
$$




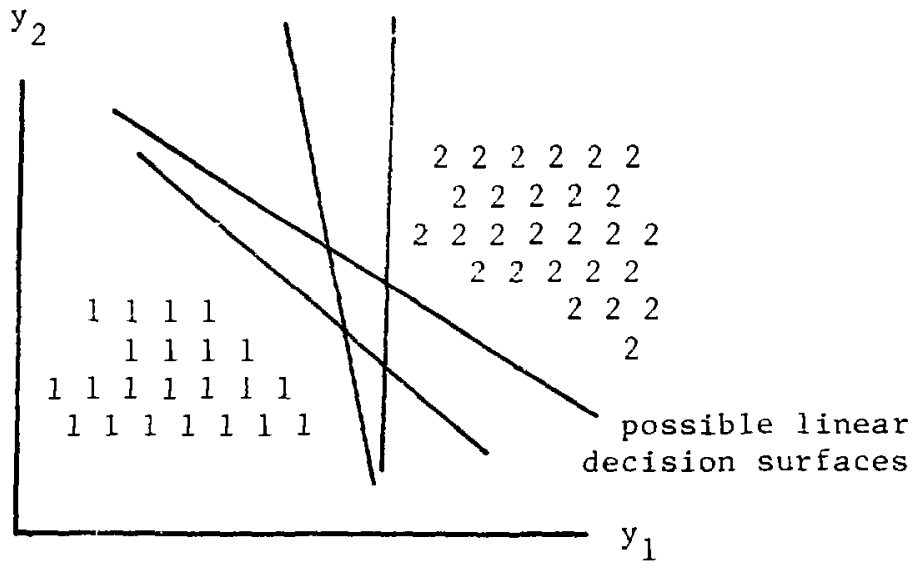

(a) Linearly Separable

$\mathrm{y}_{2}$

$z=\left(y_{1}, y_{2}\right)$ belongs to $c_{2}$

$\begin{array}{lllllllll}2 & 2 & 2 & 2 & 2 & 2 & 2 & 2 & 2\end{array}$

$\begin{array}{llllllllll}2 & 2 & 2 & 2 & 2 & 2 & 2 & 2 & 2 & 2\end{array}$

$1=\left(y_{1}, y_{2}\right)$ belongs to $C_{1}$

$\begin{array}{lllllllllll} & 1 & 1 & 1 & 1 & 1 & 1 & 2 & 2 & 2 & \text { ? }\end{array}$

$\begin{array}{lllllllll}1 & 1 & 1 & 1 & 1 & 2 & 2 & 2 & 2\end{array}$

$\begin{array}{lllllllll}1 & 1 & 1 & 1 & 1 & 2 & 2 & 2 & 2\end{array}$

$\begin{array}{llllllll}1 & 1 & 1 & 2 & 2 & 2\end{array}$

$1 \quad 2222$

2222222

$y_{1}$

(b) Non-Linearly Separable

Figure 3.9 Linearly and Non-Linearly Separable Classes 


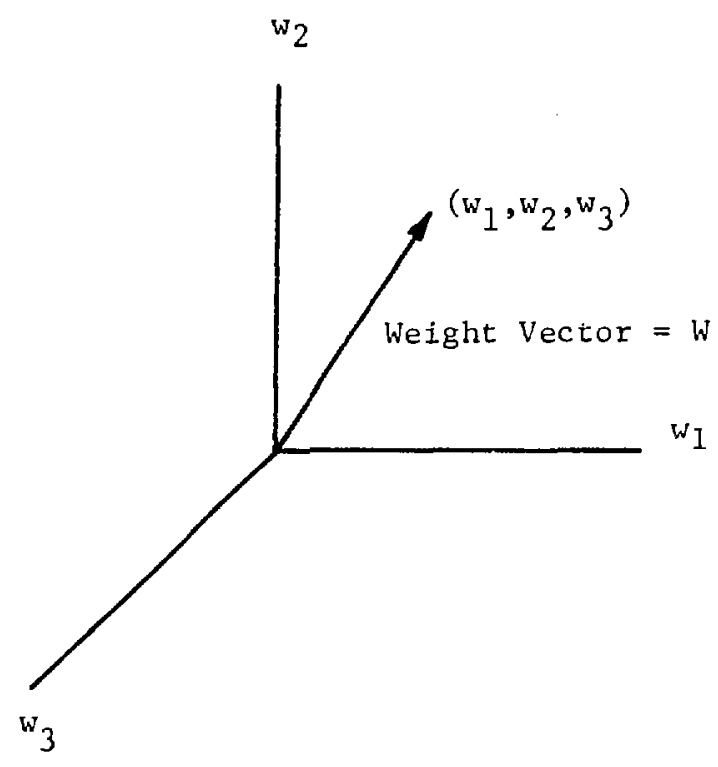

Figure 3.10 Weight Space

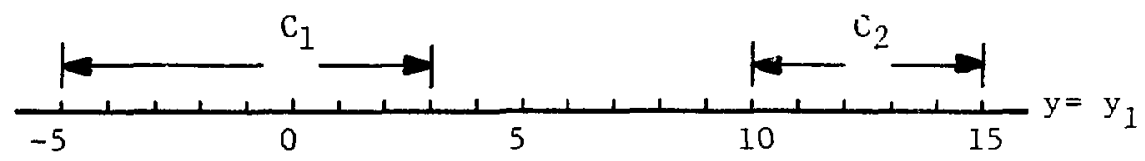

Figure 3.11 One Dimensional Feature Space 
If a decision surface is located at point $y^{\prime}$, then for this exacple

$$
\mathrm{g}(\mathrm{l})=0=\mathrm{w}_{1} \mathrm{y}^{\prime}+\mathrm{w}_{2}
$$

and

$$
\mathrm{w}_{2}=-\mathrm{w}_{1} \mathrm{y}^{\prime}
$$

The surface given by Equation 3.9 is called a pattern hyperplane in weight space and oivices veignt space into two regions: (1) that region for winich $g=\left(w_{1} y^{\prime}+w_{2}\right)>0$ and (2) that region for which $g=\left(w_{1} y^{\prime}+w_{2}\right)<0$. Pattern hypezplanes for $y^{\prime}=2,3$, and 10 are shown in Figure 3.12 where the + and signs indicate the sign of $g$ on the different sides of the hyperplane. The shaded region of Fisure 3.12 is that region of weight space for which

$$
g>0 \text { if } y^{\prime} \geq 10
$$

and

$$
g<0 \quad \text { if } y^{\prime} \leq 3
$$




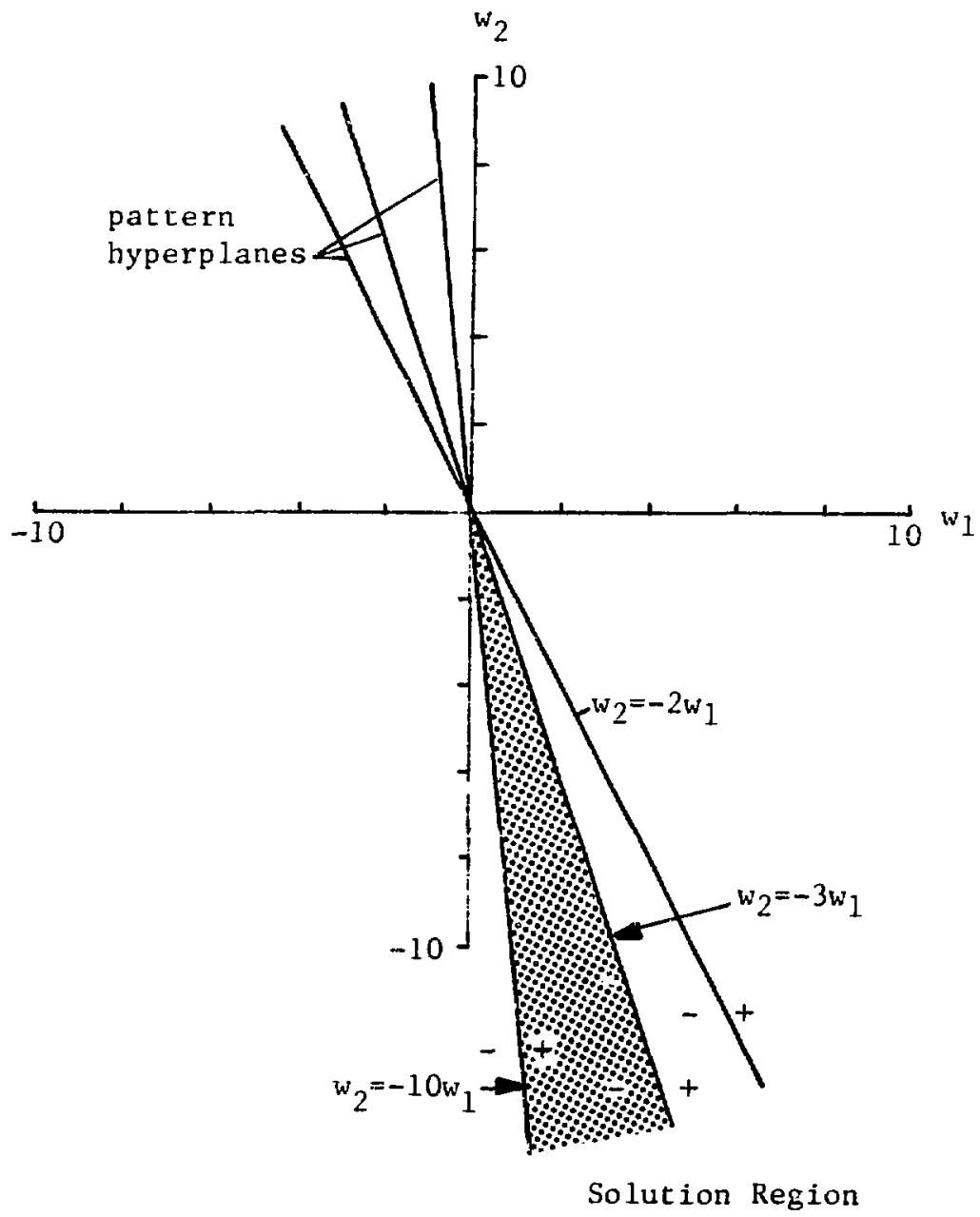

Figure 3.12 Pattern Hyperplanes in Weight Space 
and is called the solution region. Any $h$ in the solution region results In a discriminant function which satisfies Equation 3.3.

The training of a linear classifier consists of first guessing an initial reight vector, $H_{1}$. The classifier is then presented with procotspes, $Y_{i}$, of known classizication. If $g\left(Y_{i}\right)$ gives the correct classizication for $Y_{i}, W$ is unchanged. If $g\left(Y_{i}\right)$ gives tire ircorrect classification then $W$ is corrected as follows:

$$
\text { if } \begin{aligned}
g\left(Y_{i}\right) & >0 \text { and } Y_{i} \text { belongs to } C_{1} \\
V_{i+1} & =V_{i}-c Y_{i}^{+} \\
\text {if } g\left(Y_{i}\right) & <0 \text { and } Y_{i} \text { belongs to } C_{2} \\
h_{i+1} & =V_{i}+c Y_{i}^{*}
\end{aligned}
$$

where $c>0$

The effect of the above procedure ray be varied depending on the value of $c$, the correction increment. The above scheme will alvays move $h$ in a direction normal to the pattern hyperplane*. The size of $c$ deteraines how far the $b$ is moved. Three rules ${ }^{15,16}$ comionly used to determine the value of $c$ are:

\footnotetext{
$\overline{* i}$ is moved along the direction of the vector $\left(w_{i+1}-w_{i}\right)= \pm c r_{i}^{*}$. The eçution for all hyperplanes perpendicular to the vector ( $\therefore \cdot\left(=c *_{i}^{*}\right)=r_{1}$ or $W \cdot Y_{i}^{*}=r_{2}$ there $r_{1}$ and $r_{2}$ can be any scalar values ${ }^{35}$. lowever, thie equation of the ingperplane corresponding to the prototype $\mathrm{r}_{i}$ is $G \cdot r_{i}^{*}=0$. Therefore, using $r_{2}=0, W$ is noved nomal to the pattern hyperplane in weight space.
} 
(1) Fixed Increment Rule: c is taken to be any fixed increment greater than zero. In this case the weight adjustment may or may not correct the misclassification of the prototype, depending on the value of $\mathrm{W} \cdot \mathrm{Y}^{*}$ in relation to $\mathrm{c}$.

(2) Absolute Correction Rule: $c$ is the smallest integer greater than $|W \cdot Y *| / Y * \cdot Y *$. Thus after one adjustment with this rule $W$ will be on the correct side of the pa'tern hyperplane.

(3) Fractional Correction Rule: $c$ is chosen such that $W$ is moved a fractional distance, $\lambda$, towards the pattern hyperplane. The distance from the weight vector $W$ to the pattern hyperplane defined by $\mathrm{X}$ is given by

$$
D=\frac{\left|W^{*} Y^{*}\right|}{\left|Y^{*}\right|}=\frac{\lg (Y) \mid}{\left|Y^{*}\right|}
$$

Therefore, using

$$
\left|W_{i+1}-W_{i}\right|=c|Y *|=\lambda D
$$

c is given by

$$
c=\frac{\lambda D}{\left|Y^{*}\right|}=\frac{\lambda|g(Y)|}{\left|Y^{*} \cdot Y^{*}\right|}
$$


If $\lambda>1, W_{i+1}$ will be on the correct side of the hyperplane. Throughout this study $\lambda$ will be referred to as the learning parameter since it controls the rate of learning by the classifier. Figure 3.13 illustrates the behavior of these three rules using the problem illustrated in Figure 3.11 and the following prototypes (prototypes are presented to the classifier in the order presented below)
(1) $y=-2$ and belongs to $c_{1}$
(2) $y=10$ and belongs to $C_{2}$
(3) $y=2$ and belongs to $C_{1}$

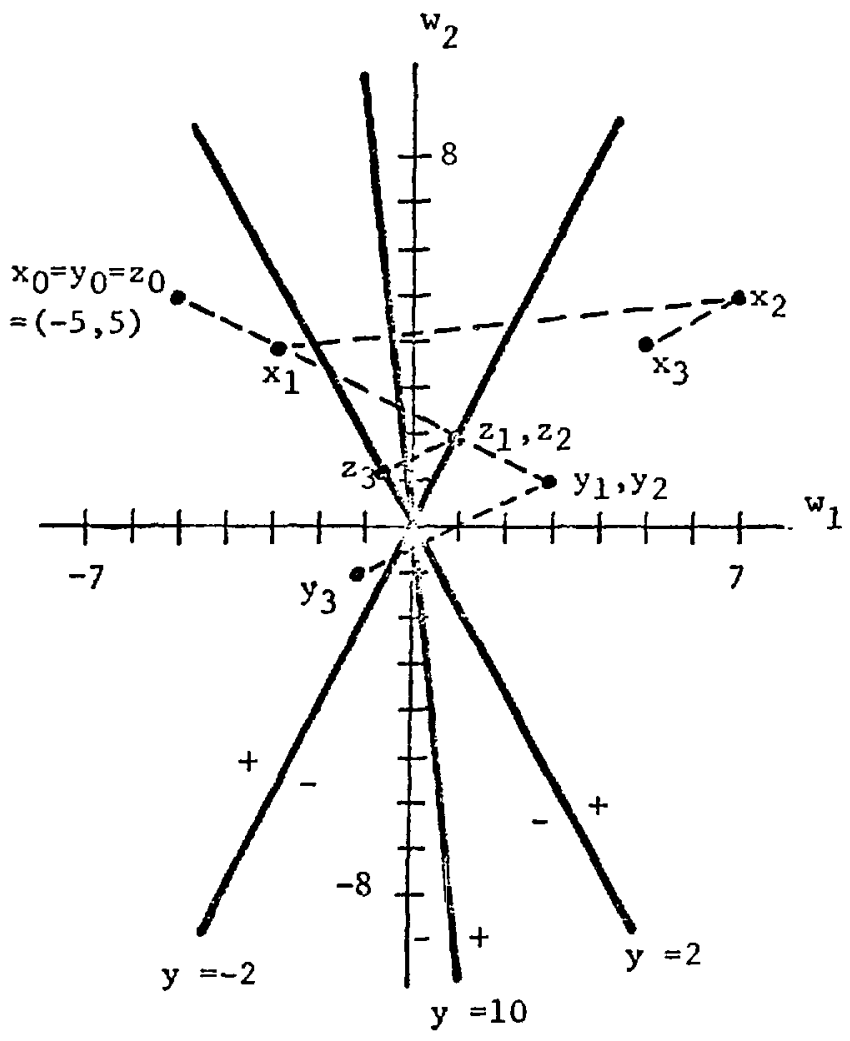

Figure 3.13 Example of the Fixed Increment, Absolute Correction, and Fractional Correction kules 
The $x_{i}, y_{i}$ and $z_{i}$ are the positions of $W$ after the $i^{\prime}$ th prototype nas been presented using the fixed increment $(c=1)$, absolute correction, and fractional correction rules $(\lambda=1)$ respectively.

\subsection{2) Quadratic Discriminant Functions}

The general form of a quadratic discriminant function for a two class problem with $\mathrm{N}$ dimensional feature vectors is given by

$$
g(Y)=\sum_{n=1}^{N} w_{n, n} y_{n}^{2}+\sum_{n=1}^{N-1} \sum_{k=n+1}^{N} w_{n, k} y_{n} y_{k}+\sum_{n=1}^{N} w_{n} y_{n}+w_{N+1}
$$

A quadratic discriminant function has $M=(N+1)(N+2) / 2$ weights. This type of $g(Y)$ can be treated in exactly the same manner as the linear $g(Y)$ if the feature vector is first operated on by a "quadric processor"15 as shown in Figure 3.14. The quadric processor behaves as a feature seleztor except that the dimensionality of the data is increased from $N$ to $M$ instead of decreased. The same techniques described in Section 3.2.1 can be used to learn the $W$ vector corresponding to $g(F)$. This same procedure can also be performed for any $g(Y)$ which depends linearly on the $w_{1}, w_{2} \ldots w_{M}$ resulting in 


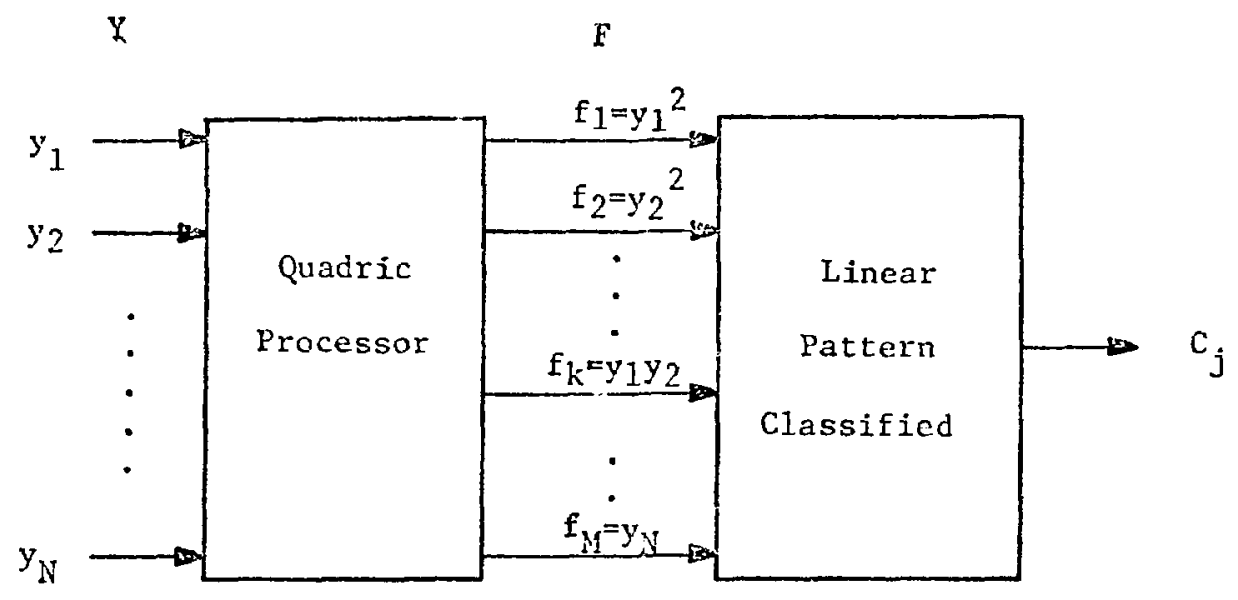

Figure 3.14 Quadratic Discriminant Functions

$$
g(Y)=w_{1} f_{1}(Y)+w_{2} f_{2}(Y)+\ldots+w_{M} f_{M}(Y)+w_{M+1}
$$

Such a $g(Y)$ is frequently referred to as a $\phi$ function ${ }^{15}$.

\section{3) Sequential Statistical Classification Techniques (Case E)}

The main incentive for using a statistical approach in pattern classification is that many processes can best be characterized in statistical terns. It is also often desirable to evaluate a pattern classifier in terms of its statistical performance. The statistical 
classifier investigated in this research is similar to the deterninistic classifier described in Section 3.2 in that a form of $g(Y)$ is assumed (i.e. $g(Y)=f(W, Y))$. The following notation will be used when referring to the statistical nature of pattern classifiers:

$$
\begin{aligned}
\mathrm{p}\left(\mathrm{Y} \mid \mathrm{C}_{i}\right)= & \text { the probability density function of those vectors } \\
& Y \text { which belong to } C_{i} \\
\mathrm{P}\left(\mathrm{C}_{i}\right)= & \text { the probability of class } C_{i} \text { occurring }\left(\mathrm{P}\left(\mathrm{C}_{1}\right)+\mathrm{P}\left(\mathrm{C}_{2}\right)=1\right) \\
\mathrm{p}(\mathrm{Y})= & \mathrm{p}\left(\mathrm{Y} \mid \mathrm{C}_{1}\right) \mathrm{P}\left(\mathrm{C}_{1}\right)+\mathrm{p}\left(\mathrm{Y} \mid \mathrm{C}_{2}\right) \mathrm{P}\left(\mathrm{C}_{2}\right)=\text { the probability density } \\
& \text { of } \mathrm{Y} \\
\mathrm{P}\left(\mathrm{C}_{\mathrm{i}} \mid \mathrm{Y}\right)= & \mathrm{p}\left(\mathrm{Y} \mid \mathrm{C}_{i}\right) \mathrm{P}\left(\mathrm{C}_{i}\right) / \mathrm{p}(\mathrm{Y})=\text { the protability of the vector } \\
& Y \text { belonging to class } C_{i} \text {. }
\end{aligned}
$$

\subsection{1) Linear Discriminant Functions}

In Section 3.2.1 the feature vectors were assumed to be 1inearly separable. By use of statistical techniques, linear discriminant functions (i.e. $g(Y)=W \cdot Y^{*}$ ) can be used with non-linearly separable data in a least error sense.

Let the function $S\left(W, Y, C_{i} \mid C_{k}\right)$ be defined as the loss incurred ${ }^{16}$ when a pattern or feature vector, $Y$, actually belonging to class $C_{k}$ is placed in class $C_{i}$ (note that $S$ is a function of the weight vector $W$ ). A vector $Y$ is said to belong to class $C_{k}$ if 


$$
p\left(C_{k} \mid Y\right)>p\left(C_{i} \mid Y\right) \quad \text { for } i \neq k \text {. }
$$

This loss function provides a means of weighing specific classification errors nore heavily than others. For example the distance fron a misclassified prototype to the decision surface (see Figure 3.15) as given by

$$
\mathrm{d}(\mathrm{W}, \mathrm{Y})=\frac{\lg (\mathrm{Y}) \mid}{\left|\mathrm{W}^{\prime}\right|}
$$

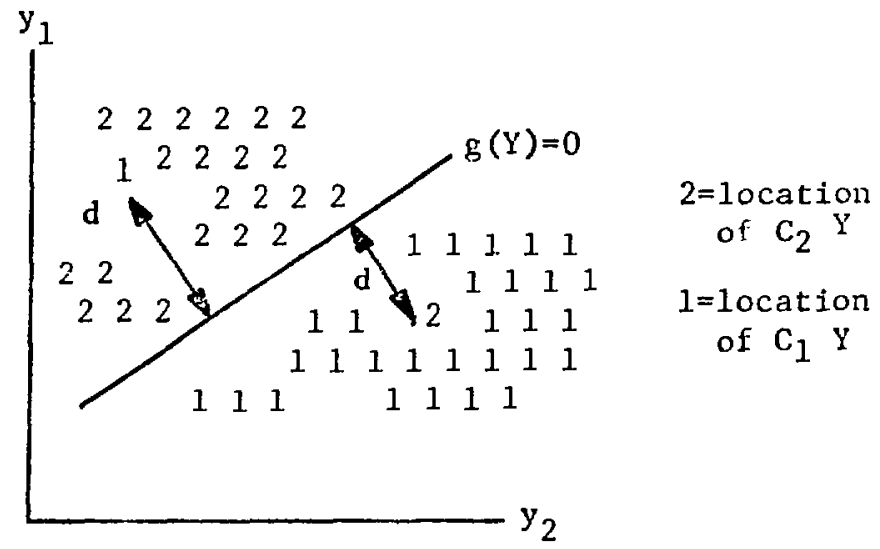

Figure 3.15 Misclassification Distance

where $W^{\prime}$ is the weight vector $W^{\prime}$ with $w_{N+1}=0$, is frequently used as a loss function ${ }^{28}$ which for two classes results in 


$$
\begin{aligned}
& S\left(W, Y, C_{1} \mid c_{2}\right)=s\left(d, C_{1} \mid C_{2}\right)=d(W, Y), \\
& S\left(W, Y, C_{1} \mid C_{1}\right)=s\left(d, C_{1} \mid C_{1}\right)=0, \\
& S\left(W, Y, C_{2} \mid C_{2}\right)=s\left(d, C_{2} \mid C_{2}\right)=0, \text { and } \\
& S\left(W, Y, C_{2} \mid C_{1}\right)=s\left(d, C_{2} \mid C_{1}\right)=d(W, Y) .
\end{aligned}
$$

The variable $d$ is frequently referred to as the "misclassification distance"128 and should not be confused with the distance, D, as given by Equation 3.11. The average $\operatorname{loss}^{16} \mathrm{~L}\left(\mathrm{~W}, \mathrm{Y}, \mathrm{C}_{\mathrm{i}}\right)$ as given by

$$
L\left(W, Y, C_{i}\right)=S\left(W, Y, C_{i} \mid C_{k}\right) p\left(C_{i} \mid Y\right) \quad \begin{aligned}
& i=1,2 \\
& k=1,2 \\
& i \neq k
\end{aligned}
$$

can be interpreted as the average $S\left(W, Y, C_{i} \mid C_{k}\right)$ associated with vector $Y$ and class $C_{i}$. If $L\left(W, Y, C_{i}\right)$ is integrated over all feature space, the result is the risk $^{16}$ associated with each class:

$$
\begin{array}{ll}
R\left(W, C_{i}\right)=\int L\left(W, Y, C_{i}\right)_{P}(Y) d Y & \begin{array}{l}
i=1,2 \\
k=1,2 \\
i \neq k
\end{array} \\
R\left(W, C_{i}\right)=\int S\left(W, Y, C_{i} \mid C_{k}\right) p\left(C_{i} \mid Y\right) p(Y) d Y &
\end{array}
$$


The total risk in the classification problem is the sum of the risks involved in each class:

$$
R(W)=R\left(W, C_{1}\right)+R\left(W, C_{2}\right)
$$

The purpose of the pattern classifier is to minimize the risk with respect to $W$. Assuming $R(W)$ is differentiable and has a global minimum with respect to $W$, the optimum $W$ is the solution of $\nabla R(W)=0$. However, as seen by

$$
\begin{aligned}
& \nabla R(W)=\nabla / p(Y) L\left(W, Y, C_{I}\right) d Y+\nabla / p(Y) L\left(W, Y, C_{2}\right) d Y \\
& =P\left(C_{1}\right) \int \mathrm{P}\left(\mathrm{Y} \mid \mathrm{C}_{1}\right) \nabla \mathrm{S}\left(\mathrm{W}, \mathrm{Y}, \mathrm{C}_{1} \mid \mathrm{C}_{2}\right) d \mathrm{Y} \\
& +\mathrm{P}\left(\mathrm{C}_{2}\right) \int \mathrm{P}\left(\mathrm{Y} \mid \mathrm{C}_{2}\right) \nabla \mathrm{s}\left(\mathrm{W}, \mathrm{Y}, \mathrm{C}_{2} \mid \mathrm{C}_{1}\right) \mathrm{dY} \text {, }
\end{aligned}
$$

this requires that $\mathrm{p}\left(\mathrm{Y} \mid \mathrm{C}_{i}\right)$ be known. This problem can be alleviated if $R(W)$ is approximated by a summation over prototypes

$$
\tilde{R}(W)=\sum_{k=1}^{2} \frac{1}{M_{k}} \sum_{n=1}^{N_{k}} s\left(W, Y_{n}^{k}, c_{i} \mid c_{k}\right) \quad \begin{aligned}
& i=1,2 \\
& k=1,2 \\
& k \neq i
\end{aligned}
$$




$$
\begin{aligned}
& \text { where } \mathrm{M}_{\mathrm{k}}=\text { no. of prototypes in class } \mathrm{C}_{\mathrm{k}} \\
& \mathrm{Y}_{\mathrm{n}}^{\mathrm{k}}=\text { nith misclassified prototype of class } \mathrm{k} \\
& \mathrm{N}_{\mathrm{k}}=\text { number of misclassified prototypes in } \mathrm{C}_{\mathrm{k}}
\end{aligned}
$$

rather than an integration over densities. Using this approximation for $R(W), W$ can be incremented proportional to the negative of $\nabla \vec{k}(W)$ as given by

if $g\left(Y_{i}\right)>0$ and $Y_{i}$ belongs to $C_{1}$

or

if $g\left(Y_{i}\right)<0$ and $Y_{i}$ belongs to $C_{2}$

then

$W_{i+1}=W_{i}-\lambda \nabla \hat{R}(W)$

$$
\nabla=\left[\begin{array}{c}
\frac{\partial}{\partial w_{1}} \\
\frac{\partial}{\partial w_{2}} \\
\cdot \\
\frac{\partial}{\partial w_{N+1}}
\end{array}\right]
$$


where $\lambda$ is a proportionality constant or learning parameter similar to the $\lambda$ described in Section 3.2.1 and

$$
\nabla \tilde{R}(w)=\sum_{k=1}^{2} \frac{1}{M_{k}} \sum_{n=1}^{N_{k}} \nabla S\left(W, Y_{n}^{k}, c_{i} \mid C_{k}\right) \quad \begin{aligned}
& i=1,2 \\
& k=1,2 \\
& k \neq i
\end{aligned}
$$

3.3.2) Quadratic Discriminant Functions

Quadratic discriminant functions using statistical techniques are treated the same as linear discriminant functions except that the feature vector is first processed by the quadric processor described in Section 3.2 .2 .

\section{4) Feature Selection}

The objective of feature selection is to retain that information necessary for classification and to eliminate that information which is not. Feature selection of ten results in greatly reducing the demands on the classifier. For example, a feature selector may process non-linearly separable data into linearly separable data as shown in Figure 3.16 . 

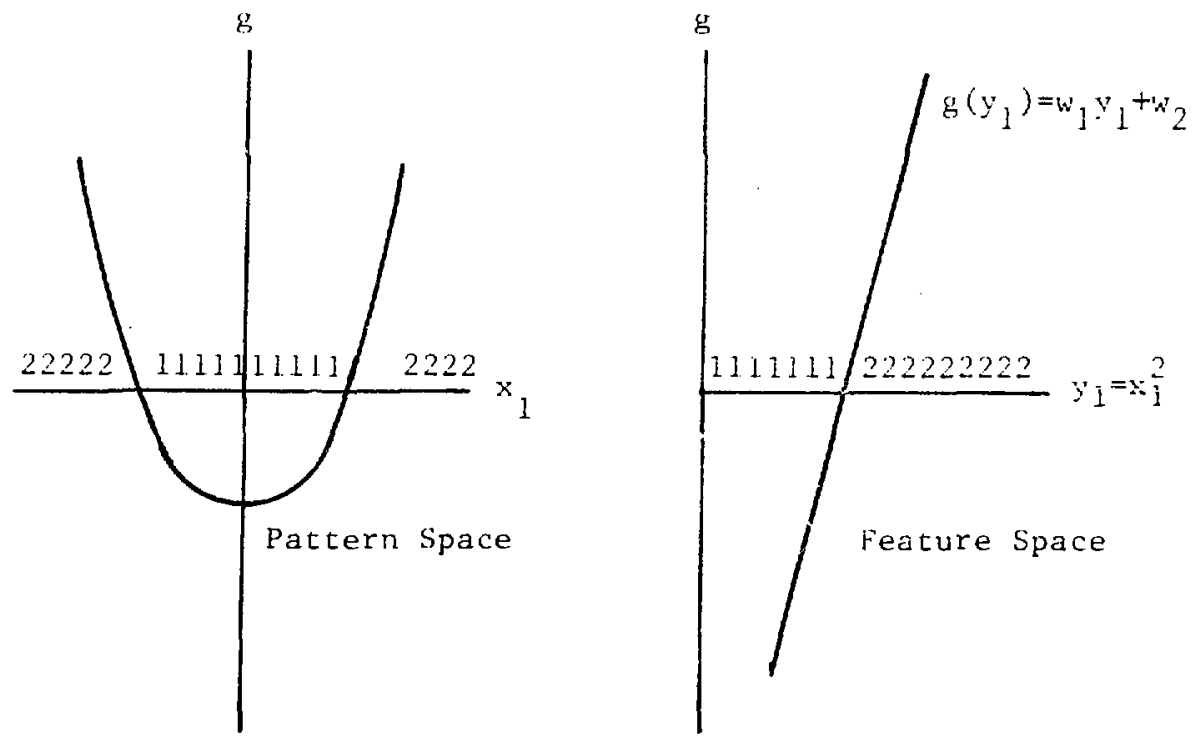

Figure 3.16 Linearization by Feature Selection

Unfortunately, the operation of feature selection is far less defined mathematically than that of pattern classification. Although a human can implement feature selection with ease, the techniques used are heuristic in nature and usually highly problem dependent. At the present time, selection decisions trivial to a human may take a great deal of effort to model and even then may take a large amount of computer time to implement. Thus tris research will rely upon the heuristic techniques of the user to supply the feature selection process. 
The pattern space of a particle transport problem consists at most of eight basic parameters:

$$
\begin{aligned}
& \text { - three position parameters }(x, y, z) \\
& \text { - three angular parameterst* }(u, v, w) \\
& \text { - energy (E) } \\
& \text { - time (t) }
\end{aligned}
$$

Certainly, if a problem independent of time is under investigation it is

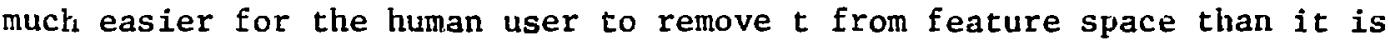
for a computer based selection system to recognize that there is no correlation between time and classification. Another case is a problem involving spherical symmetry in the geometry in which three variables $(x, y, z)$ can be replaced by one, $r\left(r=x^{2}+y^{2}+z^{2}\right)$. This not only reduces the dimensionality of the problem but can also linearize the feature space. Such a substitution is easily specified by the user but would take numerous operations to recognize computationally.

\section{5) Multiclass Problems}

The previous sections have considered pattern recognition problems involving two classes. In this research discriminant functions are learned for two classes at a time* resulting in a single discriminant finction for classes $i$ and $i+1$ (see Equation 3.2)

\pm The reason for this will become apparent in Chapter $V$. ** Only two of the angular parameters are independent. 


$$
g_{i, i+1}(Y)=g_{i+1}(Y)-g_{i}(Y) \quad i=1, \ldots, J-1
$$

where $\mathrm{J}$ is the total number of classes. These classes are ordered such that

$$
\begin{aligned}
& \text { if } g_{i, i+1}(Y)<0, \\
& \text { then } Y \text { must belong to a class } C_{j} \text { where } j=1, \ldots, i \\
& \text { if } g_{i, i+1}(Y)>0 \\
& \text { then : must velong to a class } C_{j} \text { where } j=i+1, \ldots, J
\end{aligned}
$$

Because of this characteristic, the class of a prototype can be determined as shown by the flow diagram in Figure 3.17 . 


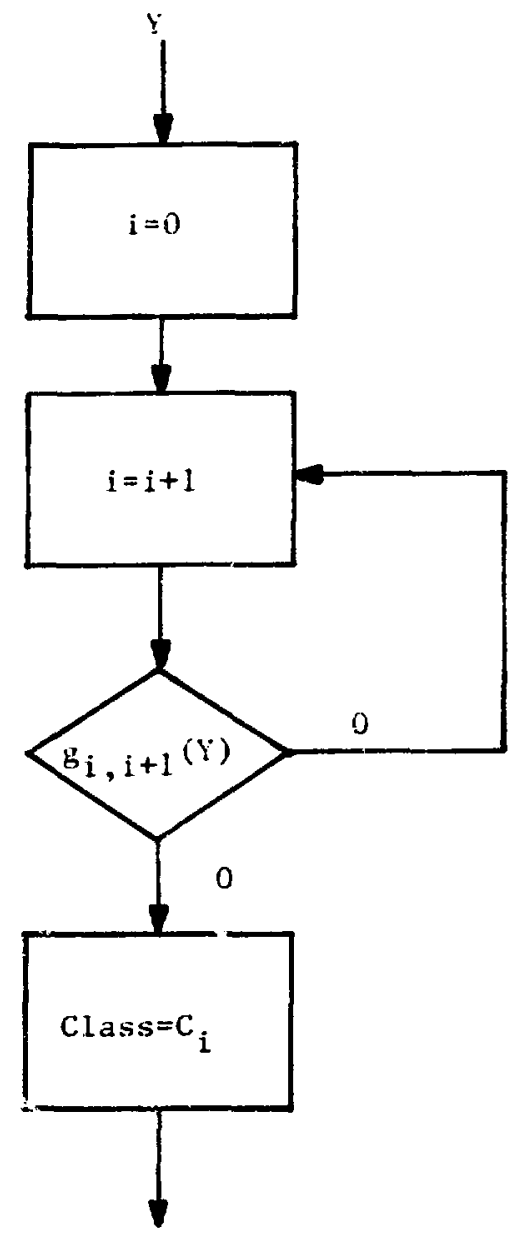

Figure 3.17 Multi-Class Problems 


\section{Recognition of Splitting Surfaces}

In Section 2.4 splitting in state space was described and it was suggested that pattern recognition be used for the Identification of the splitting surfaces. It is the purpose of this chapter tu describe how pattern recognition can be implemented and to investigate the performance of the technique.

Since the purpose of this dissertation is to demonstrate proof of principle, the technique is not applied to a general purpose Monte Carlo code. The Monte Carlo problems investigated in this research have been chosen for their simplicity and their minimal use of computer time. These sample problems include: (1) a one-dimensional oneregion homogeneous slab, (2) a one-dimensional multi-region slab, and (3) a two-dimensional, multi-region slab. These problems illustrate the basic treatment of distance and direction variables in a Monte Carlo problem.

This chapter is concerned only with the learning of the splitting surface. A description of how to use the splitting surface and what surfaces are desirable as splitting surfaces is given in Chapter $V$.

Section 4.1 describes in general how pattern recognition is used to identify splittir.g surfaces. In Section 4.2 both deterministic (see Section 3.2) and statistical (see Section 3.3) classifiers are used to Identify splitting surfaces for a one-region slab Monte Carlo problem and studies are made to determine: (1) the effects of slab thickness and class overlapping, (2) the improvement due to the use of 
buffer zones,(3) the best choice of a loss function (see Section 3.3.1) for the statistical classifier, (4) compucer time spent for pattern recognition, and (5) the sensitivity of the classifiers to the learning parameter $\lambda$. In Section 4.3 the same classifiers developed in. Section 4.2 are used to identify surfaces for multi-region problems and again the sensitivity to $\lambda$ is investigated. Section 4.3 also includes a study of the sensitivity of the classifiers to the selection of initial conditions (i.e., the initial guess for $w$ ). Section 4.4 fincreases the pattern space to two dimensions, distance and angle, thus requiring normalization of the feature vector. Studies of the learning parameter and initial conditions are then repeated for the two-dimensional problem. Section 4.5 summarizes the results of the chapter.

4.1) Bastc Principles

The purpose of this section is to relate the pattern recognition system described in Chapter III to the problem of identifying Monte Carlo splitting surfaces as described in Chapter II.

In Saction 3.1.2, the concept of "prototypes" or "training sets" was introduced. This concept is very important in this research and is discussed with respect to Monte Carlo calculations in Section 4.1.1.

Although feature selection is not discussed until Chapter $v$, Section 4.1.2 does describe how feature selection relates to the simple Monte Carlo problems of this chapter. 
Two pattern classification systems are investigated in this chapter: (1) the Case D deterministic classifier using tr:- fractional correctior rule (see Section 3.2) and (2) the Case E statistical classifier (see Section 3.3). The structure of these classifiers and the operations performed for learning a splitting surface are described In Section 4.1.3.

In Chapter III, the term "learning parameter" was introduced for both deterministic and statistical classifiers. This parameter, $\lambda$, plays an important role in this research. Its importance is explained in Section 4.1.4.

In order to evaluate different classifiers and classifier parameters, one must be able to measure the performance of the classifier. The performance measures used in this dissertation are described in Section 4.1.5.

\subsection{1) Prototypes from Monte Carlo Calculations}

Prototypes were described in Section 3.1 .2 as being pattern vectors with known classification. The prototypes allow Case $B, D$, and $F$ classifiers to learn a discriminant function, $g(Y)$, (see section 3.1.1) which is necessary before classification can take place.

In Section 3.14 it was stated that for the general Monte Carlo problem pattern space consists of 8 variables:

- three position parameters $(x, y, z)$

- three direction parameters $(u, v, w)$ 
- energy ( $E$ )

- time $(t)$

As a particle travels through a material region, it undergoes numerous collisions. At each collision point a new set of $(x, y, z, u, v, w, E, t)$ is calculated for the particle (see Appendix A). This new set of values consists of a point in state space (see Section 2.4) and can be represented by the state space vector $X$ as given by

$$
X=\left[\begin{array}{c}
x \\
y \\
z \\
w \\
v \\
w \\
E \\
t
\end{array}\right]
$$

This vector $X$ is also a pattern vector; therefore,

$$
\text { state space vector } \equiv \text { pattern vector }
$$

and

$$
\text { state space } \equiv \text { pattern space. }
$$

Thus pattern vectors are created in a Monte Carlo problem wherever a particle undergoes a coilision.

Before the pattern vectors can be used as prototypes, their classification must be known. In Section 3.5 it was stated that discriminant functions are learned for two classes at a time. Therefore, before the pattern vector, $X$, can be used as a prototype, it must be known to which class, $C_{1}(i=1,2)$, the vector belongs. This is done by Introducing the concept of "Importance". 
In this research the Importance of the vector $X$ in state space is defined as the average contribution to the tally by particles which pass through $\mathrm{X}$ divided by the average weight (see Section 2.2) that particles have at $X$. If the importance were known for all $\mathrm{X}$, there would be little reason to solve the Monte Carlo problem since the average importance of the source particles would be equivalent to the desired tally. Therefore, only approximations to the importance as defined above will be used. The approximation to the importance at $X$ of a sirigle particle passing through $X$ is given by

$$
\begin{aligned}
& I(X)=\frac{T}{W t(X)}, \\
& \text { where } T=\text { the contribution of the particle to the tally } \\
& W t(X)=\text { the weight of the particle when it existed at } X .
\end{aligned}
$$

Using Equation 4.1 for the importance, one can classify the pattern vector $\mathrm{X}$ as follows:

$$
\begin{aligned}
& \text { if } I(X)<\bar{I}, X \text { belongs to } c_{1} \\
& \text { if } I(X)>\bar{I}, X \text { belongs to } c_{2} \text {, }
\end{aligned}
$$


where $\bar{I}$ is an importance which is used to discriminate between $c_{1}$ and $\mathrm{C}_{2}$ ( $\overline{\mathrm{I}}$ will be discussed later).

Thus prototypes, $x$, are created at collision points with their classification determined by Equation 4.2. The following example illustrates the creation of prototypes.

Example: Consider the problem of the one-dimensional homogeneous slab shown in Figure 4.1 with a unidirectional source at $x=0$ and a tally of particles as they cross the surface at $x=L$. A single particle is shown traversing the slab and undergoing five collisions before it is tallied. The absorption probability at each collision is .2; thus, the weight of the particle is multiplied by .8 at each collision. When the particle is tallied approximations of the importances at the various collision points can be found by

$$
I(x)=\frac{W t(L)}{W t(x)}
$$

where

$$
\begin{aligned}
I(x)= & \text { importance of a particle at } x \\
\text { Wt }(L)= & \text { tally contribution of the particle= } \\
& \text { weight of the particle at } x=L \text {, and } \\
\text { Wt }(x)= & \text { weight of the particle at } x .
\end{aligned}
$$


The values of $I(x)$ for the five collisions shown in Figure 4.1 are given in Table 4.1 . If $\bar{I}=.75$, the pattern vectors are classified as shown in Table 4.1.

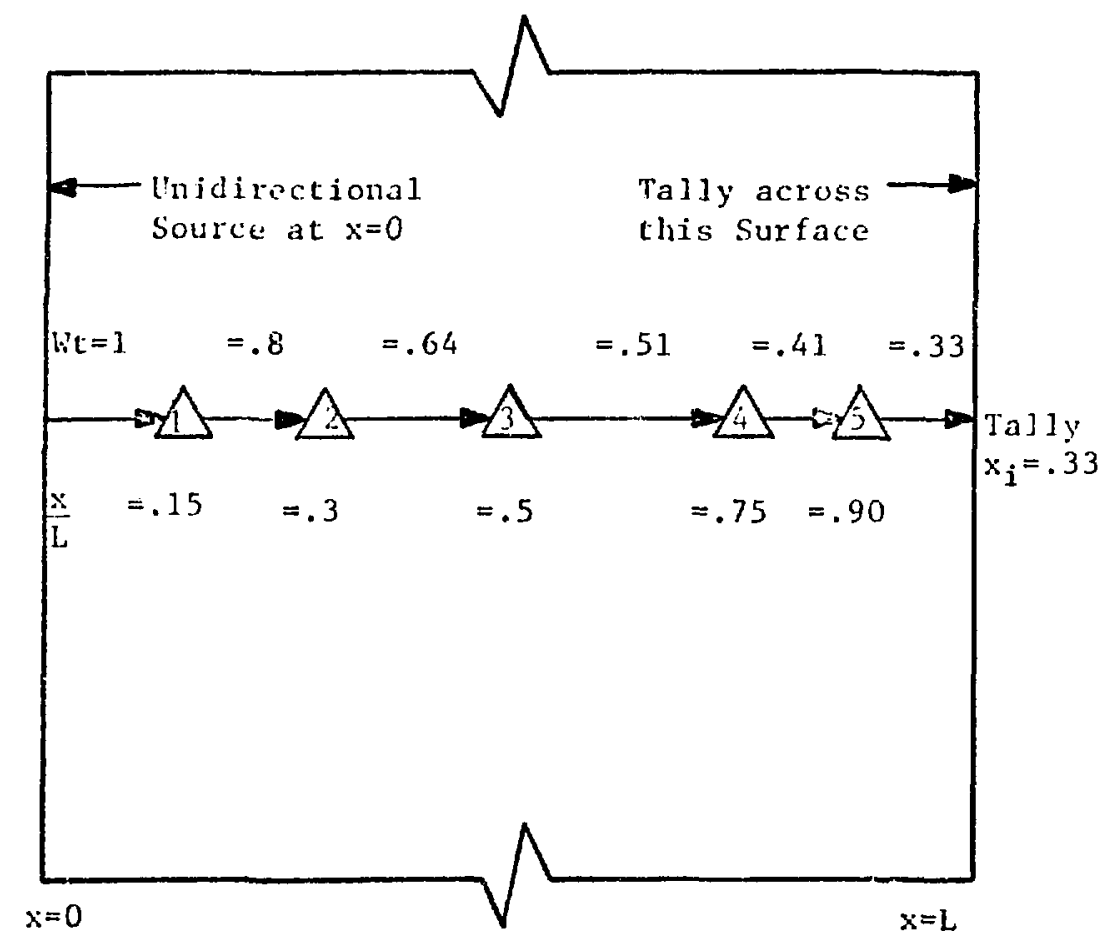

Figure 4.1 Prototypes from a One-dimensional slab Monte Carlo Problen 


\subsection{2) Feature Selection}

Sections 4.2 and 4.3 use one dimensional slab Monte Carlo problem and thus pattern space consists of the single variable $x$, where $\mathrm{x}$ is the distance from the source (see Figure 4.1). For this problem feature space will be the same as pattern space; thus

$$
X=\left[x_{1}\right]=Y=\left[y_{1}\right]=x .
$$

The two dimensional problem of Section 4.4 will be treated similariy. In this case

$$
X=\left[\begin{array}{l}
x_{1} \\
x_{2}
\end{array}\right]=Y=\left[\begin{array}{l}
y_{1} \\
y_{2}
\end{array}\right]=\left[\begin{array}{l}
x \\
\phi
\end{array}\right]
$$

Table 4.1 Prototypes for Problem Illustrated in

\begin{tabular}{|c|c|c|c|}
\hline$\frac{x}{L}$ & Wt $(x)$ & $\underline{I(x)}$ & $\mathrm{C}_{1}$ \\
\hline .15 & .8 & .41 & 1 \\
\hline .30 & .64 & .51 & 1 \\
\hline .50 & .51 & .64 & 1 \\
\hline .75 & .41 & .8 & 2 \\
\hline 90 & .33 & 1.0 & \\
\hline
\end{tabular}
Figure 4.1 and $\bar{I}=.75$ 
where $\phi$ will be defined in Section 4.4 . Some experiments are also made in Section 4.4. for which $X \neq Y$.

\subsection{3) The Pattern Classifier}

The classiffers used in this research assume a linear form for $g(Y)$ as described in Sections 3.2 .1 and 3.3 .1 and operate in two stages:

(1) prototypes are used to learn the weight vector $W$ for the linear discriminant function $g(Y)=W \cdot Y *$ (see Equation 3.8)

(2) the discriminant function $g(Y)$ is used to classify the feature vector $Y$ where $Y$ is of unknown classification. This chapter is concerned only with the first operation. The second operation is discussed in Chapter $\mathrm{V}$.

The learning of the weight vector, $W$, consists of :

(1) selecting an initial value of $t$ and

(2) incrementing $W$ (using Equations 3.10 and 3.20) whenever a prototype belonging to class $C_{i}$ (as determined by Equation 4.2$)$ is classified into $C_{j}(j \neq i)$ as determined by the sign of $g(W)$.

The sensitivity of the classifier to the initial selection of $W$ is investigated in Section 4.3 .1 .

The incrementing of $W$ is the major operation of the learning process. The classification of a feature vector according to Equation 4.2 is referred to as the "teacher". The classification according to 
the pattern classifier is determined by Equation 3.3 which is repeated here as

$$
\begin{array}{ll}
\text { if } g(Y)<0 & Y \text { belongs to } C_{1} \\
\text { if } g(Y)>0 & Y \text { belongs to } C_{2}
\end{array}
$$

The classification according to Equation 3.3 is referred to as the "student". Thus whenever the student disagrees with the teacher, the student is corrected by adjusting $w$. This process continues until the agreement between student and teacher meets some threshold value. At this point the classifier has learned the desired $W$. The intersection of the discriminant function $g(Y)$ with state space is called the decision surface (see Section 3.1.1) and is given by $g(Y)=0$. This surface separates state space irto two regions:

(1) $X$ for which $g(Y)>0$ and (2) X for which $g(Y)<0$. Since this is tine purpose of a splitting surface, it follows that

decision surface $\equiv$ splitting surface.

These two terms will be used interchangeably throughout the remainder of this dissertation. 
The weigit acjustrent algorith using the deterministic classifier sas the fractional correction rule is given by (see Equations 3.10 ard 3.12$)$

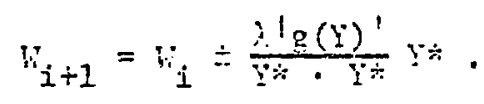

For the statistical classijier the adjustment algorithm is given by (see Eq̣uation 3.20)

$$
r_{i+1}=r_{i}-\lambda \Gamma \tilde{R}(n)
$$

In both cases the azcunt of the adjustnent of $H_{i}$ is determined by the learaing parareter, $\lambda$.

then a pattern classifier is presented with overlapping distributions: (see Section 3.1.2), the selection of an optimum $\lambda$ becores quize ccoplex. Sefore using the adjustment algorithins, the classifier aust be told to which class a feature vector belongs.

Fill cata prodiced by Yonte $C \equiv r l o$ will be overlapping since the prototype classification is deternined by Equation 4.2 and $I(X)$ is cnly a single estf:ate of the true value of the importance at $X$. Overlapping hill be ifscussed further in Section 4.2 . 
When $\mathrm{Y}$ can belong to either class with probability $\mathrm{p}\left(\mathrm{C}_{1} \mid \mathrm{Y}\right)$ and $\mathrm{p}\left(\mathrm{C}_{2} \mid \mathrm{Y}\right)$ (see Section 3.1 .2 h the classifier should be told

$$
\begin{array}{ll}
\text { if } p\left(C_{1} \mid Y\right)>p\left(C_{2} \mid Y\right) & Y \text { belongs to } C_{1} \\
\text { if } p\left(C_{2} \mid Y\right)>p\left(C_{1} \mid Y\right) & Y \text { belongs to } C_{2}
\end{array}
$$

In this research tine $\mathrm{p}\left(\mathrm{C}_{i} \mid \mathrm{r}\right)$ are unknown. As a result, when the classifier is told that $\mathrm{Y}$ belongs to $\mathrm{C}_{2}$, it may be that

$$
p\left(C_{1} \mid Y\right)>p\left(C_{2} \mid Y\right)
$$

In such a case, the classifier should not adjust the weights if $g(Y)<0$, since it is the prototype classification that is wrong, not the classifier. However, since the $p\left(C_{i} \mid y\right)$ are not known, it is impossible to determine which classification is right.

As the confidence in a prototype's classification becomes small, i.e., as

$$
\mathrm{p}\left(\mathrm{C}_{1} \mid \mathrm{Y}\right) \rightarrow .5 \text { and } \mathrm{p}\left(\mathrm{C}_{2} \mid \mathrm{Y}\right) \rightarrow .5
$$


a smaller value of $\lambda$ should be used than when the confidence becomes great, i.e., as

$$
p\left(C_{i} \mid Y\right) \rightarrow 1 \quad i=1 \text { or } 2
$$

Thus, it would be beneficial to use a $\lambda$ that is a function of

$$
\Delta(Y)=1-\left|\mathrm{P}\left(\mathrm{C}_{1} \mid \mathrm{Y}\right)-\mathrm{P}\left(\mathrm{C}_{2} \mid \mathrm{Y}\right)\right|
$$

which is not possible since $\Delta(Y)$ is unknown. If the average value of $\Delta(Y)$ is known for a problem as given by

$$
\bar{\Delta}=\frac{\int \Delta(\mathrm{Y}) \mathrm{dY}}{\int \mathrm{d} \mathrm{Y}}
$$

a "semi-optimal" constant $\lambda$ can be chosen for each problem such that

$$
\lambda \propto \bar{\Delta}
$$


For Monte Carlo problems, the value of $\bar{\Delta}$ is not known. Therefore a single $\lambda$ must be used for all problems which from the above discussion is definitely sub-optimal. One of the purposes of this dissertation is to determine the sensitivity of classifier performance to $\lambda$ so that a suitable $\lambda$ can be chosen for a large range of problems.

\subsection{5) Classifier Performance}

Two parameters are used in this dissertation to measure the performance of the various classifiers: misclassification rate and variability. In addition the classifiers are timed in order to estimate how much computer time is spent in learning a splitting surface.

A prototype is said to be misclassified if the student disagrees with the teacher (see Section 4.1.3). This can be summarized as follows:

$$
\begin{aligned}
& \text { if } I(Y)<\bar{I} \text { and } g(Y)>0 \\
& \text { or } \\
& \text { if } I(Y)>\bar{I} \text { and } g(Y)<0 \text {, }
\end{aligned}
$$

then the prototype $\Psi$ has been misclassified. The misclassification rate used in this dissertation is given by 


$$
\begin{aligned}
& \mathrm{R}_{\mathrm{NC}}=\frac{\mathrm{l}_{1}+x_{2}}{u_{1}+r_{2}}, \\
& \text { where } x_{i}=\text { rumber of protorypes belonging to } c_{i} \text { (accoring to } \\
& \text { the teacher) that are nisclassified into } c_{j}(i \neq j) \text { ard } \\
& Y_{1}=\text { nusber of prototypes belonging to } c_{i} \text { (accorcing to } \\
& \text { the teacher). }
\end{aligned}
$$

It is important to note that the above misclassification rate is that seen by the teacher. Because of this the misclassification rate can never be lower than the misclassification rate of the teacher. Tierefcre, a projlem in wich the class distributions, $p\left(C_{i} \mid Y\right)$, overlap by $30 \%$ wi:1 never have a tisclassification rate below .3. Similarly a problea in wich the $p\left(C_{i} \mid Y\right)$ have no overlap can theoretically have a nisclassizication rete of zero. Because of the Monte Carlo problem dependence of the misclassification rate another parameter, the variability, is used to measure performance.

The variability is a measure of the amount of fluctuation of the decision sliface. The decision surface after the $j$ 'th prototype for the one dimensional problem is given by

$$
s_{j+1}=\left(\frac{-w_{2}}{w_{1}}\right)_{j+1}
$$




$$
\begin{aligned}
& \text { where } s_{1}= \\
& \begin{aligned}
\left(w_{1}\right)_{j+1}= & \text { the initial selection of the decision surface } \\
& \text { exist } \text { th after the } j \text { 'th prototype } \\
W_{1}= & \text { the initial selection of } W
\end{aligned}
\end{aligned}
$$

The mean value of the decision surface after $J$ prototypes is given by

$$
\bar{s}=\frac{\sum_{j=1}^{J+1}\left(\frac{-w_{2}}{w_{1}}\right)_{j}}{J+1}
$$

The variability of the decision surface as used in this dissertation is given by

$$
\operatorname{var}=\left[\frac{\sum_{j=1}^{J+1}\left(\frac{-w_{2}}{w_{1}}\right)_{1}^{2}-\bar{s}^{2}}{J+1}\right]^{1 / 2} \times \frac{1}{\bar{s}}
$$


The variability can be thought of as the relative error of the decision surface. As the decision surface converges the variability decreases. The variability of the statistical classifier approaches zero; however, as will be seen, this is not true for the deterministic classifier.

The misclassification rate and variability can also be used by the classifier to determine when a decision surface has been learned. This can be done by first setting a threshold on the misclassification rate and variability. Once the misclassification rate threshold has been reached, the classifier continues until the variability threshold is reached. The values to use as thresholds will require experience with a general purpose Monts Carlo code; however, an indication of those values is given in this research.

Because the purpose of using pattern recognition is to save computer time, it is important to know how much computer time is used by the classifier to learn a splitting surface. This is dore in this research by using the timing routine described in Reference 36 . It should be noted that the FORTRAN programming used in this research is in no way optimized. Therefore, the timing data should be considered as an upper limit to the values that could be obtained using optimized FORTRAN or assembly language programming. It is also important to realize that the relative difference between timing values for different operations, quoted in this research could also change depending on the programming used. Thus when $i$ it is stated that operation $x$ is quicker than operation $y$, it should be remembered that this is relative to the programming techniques used in this research. 
$4.2 \quad$ One-Dimensional Cne-Pegion S1at

The Monte Carlo problem used in this section consists of calculating the transmission probability through a homogeneous slab of thickness L (see Figure 4.2). At each collision the particle's weight is reduced by the absorption probability and then alloved to continue in the forward direction only. The computer code for this problem is shown in Appendix $C$. The analytical solutions for the transmission probability to $x$ and the importance at $x$ are oiven by

$$
\begin{aligned}
& T P=e^{-\sum_{a} x} \text { and } \\
& I=e^{-\sum_{a}(L-x)} .
\end{aligned}
$$

Figure 4.3 shows the distribution of importances obtained from Monte Carlo runs of 200 particles for various thickness slabs and macroscopic cross sections of $\Sigma_{t}=.5$ and $\Sigma_{s}=.4$. The discrete behavior of this distribution can be attributed to the fact that only an integer number of collisions can occur resulting in importances given by

$$
I(x)=(.8)^{n}
$$




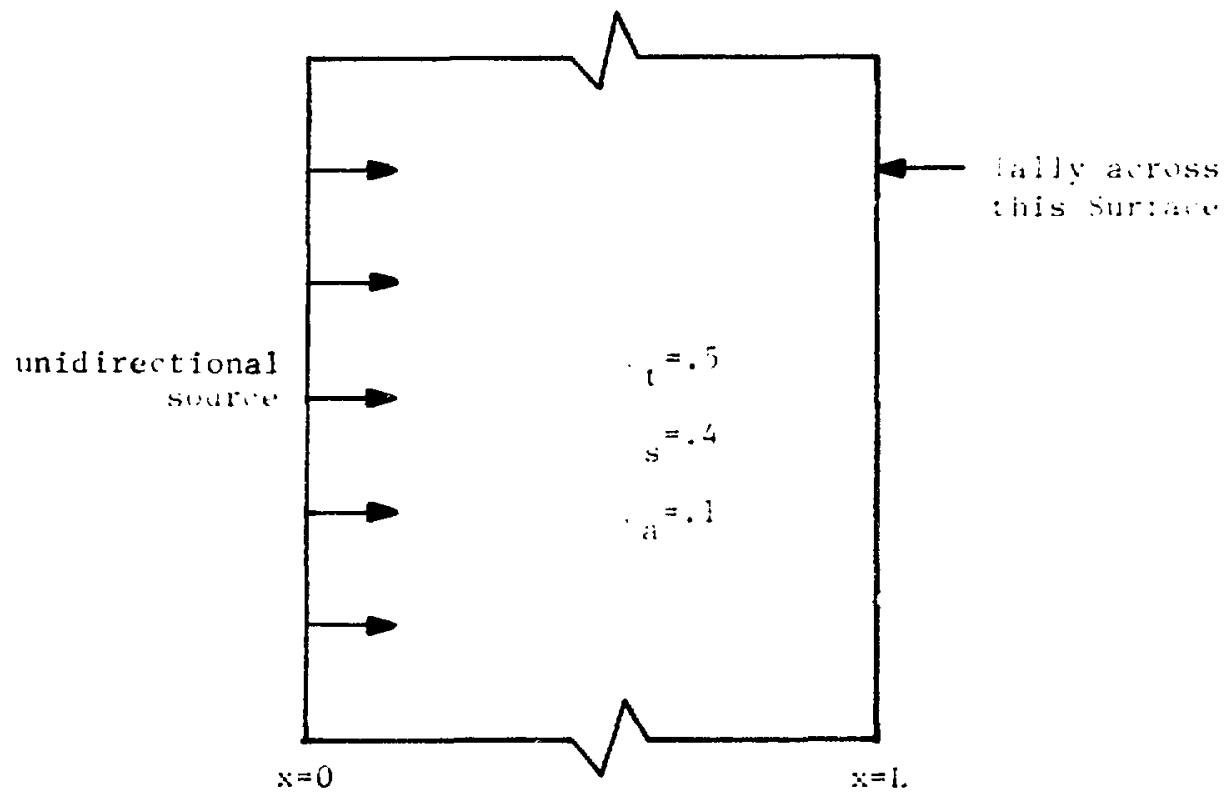

Figure A. The One-imensional, One-Region, Homgeneous slab

where $\mathrm{n}$ is th.e number of collisions a particle has undergone between the time tt leaves $x$ and is tallied. Because these importance distributions are unknown prior to the beginning of a Monte Carlo calculation, the mean or nedian must be learned during the initial stages of the run. This value can then be used as the $\bar{I}$ mentioned in section 4.1 .

Before applying. pattern recognition it is helpful to observe the probability densities, $p\left(c_{1} \mid Y\right)$, of the two classes separated by $\bar{I}$. These densities are shown in Figure 4.4 for several thickness slabs using the mean importance for $\bar{I}$ and cross sections of $\Sigma_{t}=.5$ and 

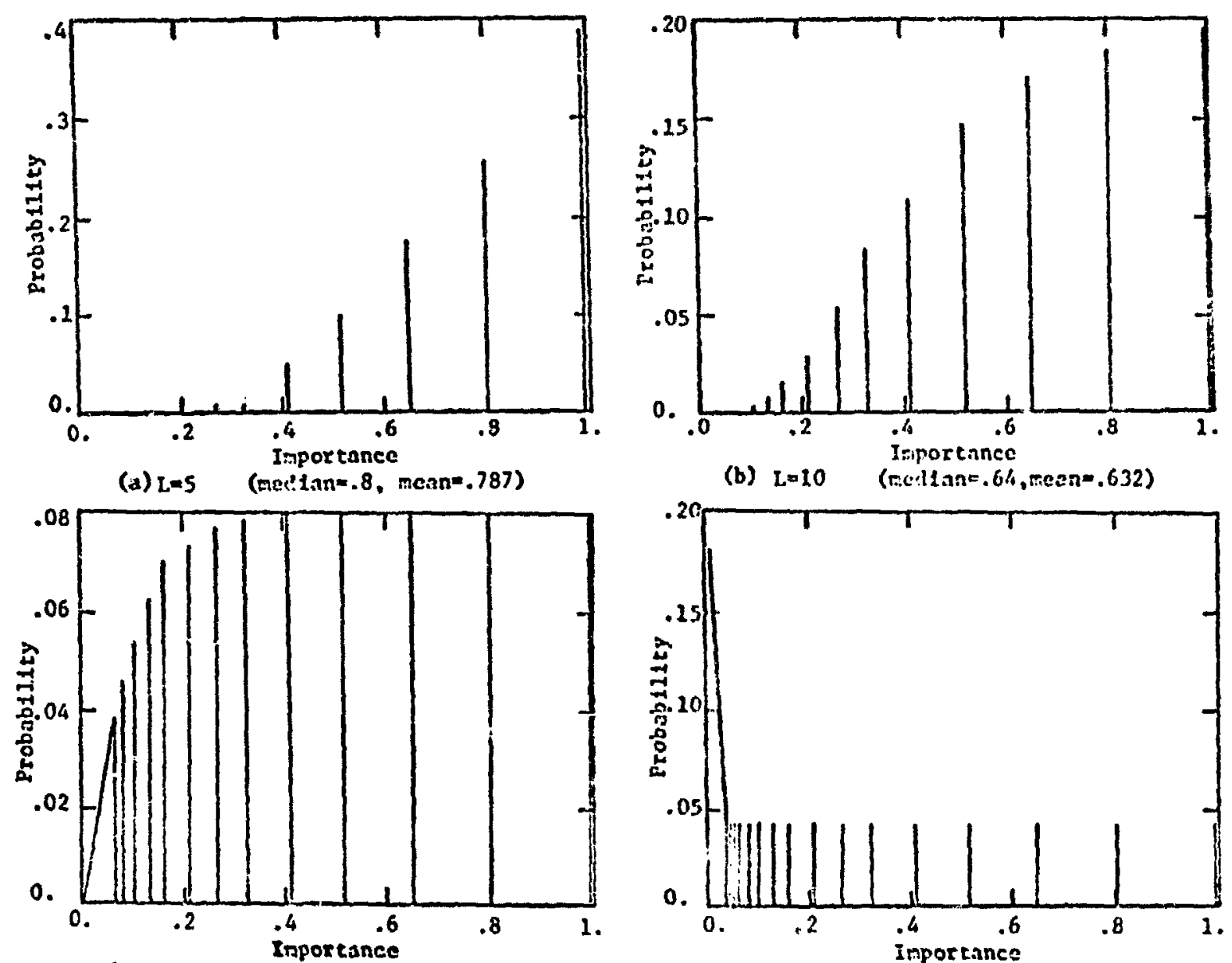

(c) $\quad L=25$

(medlan=. 2621, mean $=.367$;

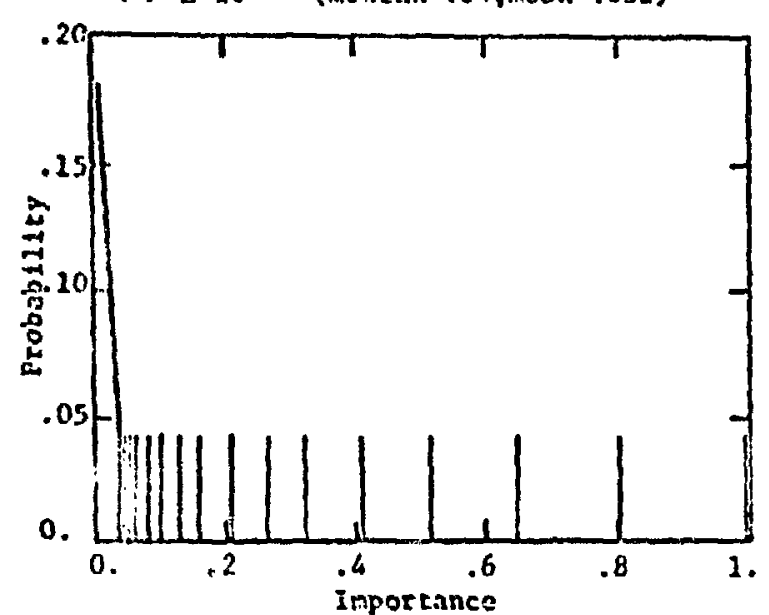

(d) Lo50 (merl Lan=.0359, meann.199)

Eigure t.3 Importance Distributions for Several slab Thicknesses 

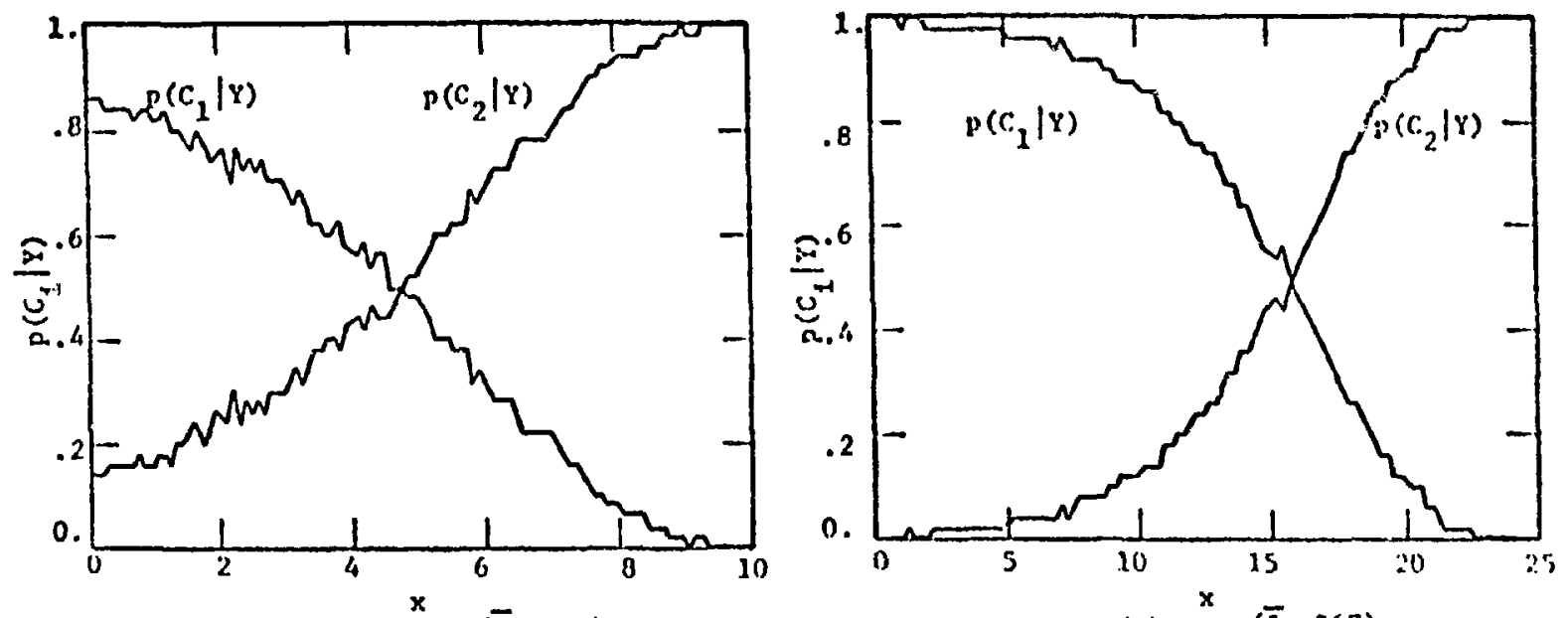

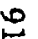
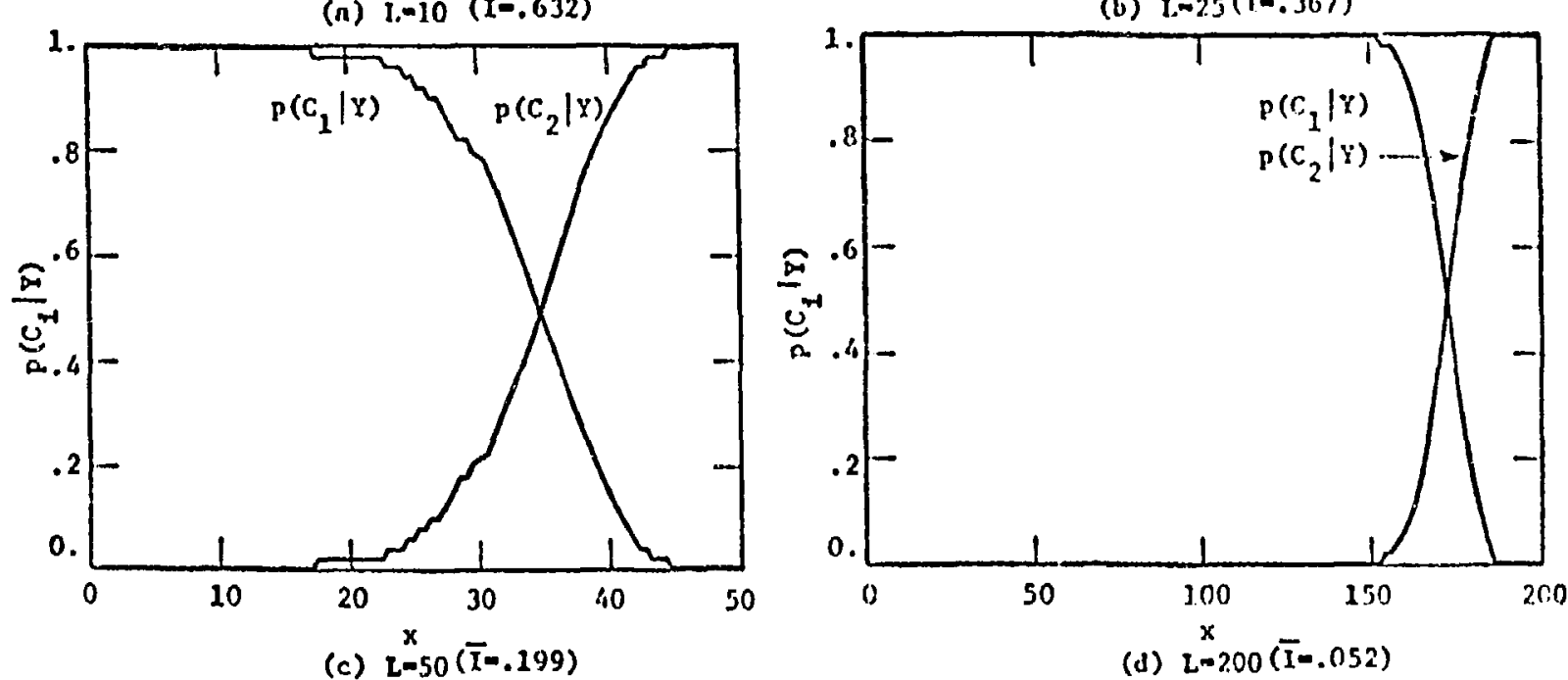

Figure 4.4 Class Distributions for Several. Sinb Thicknesses 
$\Sigma_{s}=.4$. For this problem $p(Y)$ is constant (except at $x=0$ ) since the particles are never killed but allowed to continue througt the sla: until they are tallied. Therefore, since the $P\left(C_{i}\right)$ are also constant, the $p\left(y \mid C_{i}\right)$ will have the same shape as the $p\left(C_{i} \mid Y\right)$ only different magnitudes relative to each other.

The overlapping of the class distributions is due to the fact that prototypes created at $x$ do not all have the same importance $I(x)$, but instead have a distribution of importances (with a mean importance of $\bar{I}(x)$ as is shown in Figure 4.5 for a slab with $I=10$ and values of $\mathrm{x}=0,5$, and 9). If all tra prototypes created at $\mathrm{x}$ had the same importance, $\bar{I}(x)$, then these prototypes would all belong to the same class depending on whether $\bar{I}(x)$ is greater or less than $\bar{I}$. However, since prototypes are distributed about $\bar{I}(x)$, prototypes originating from the same $x$ can belong to both classes depending on the location of $\bar{T}$ within the distribution. This point is illustrated by Figure 4.5 where the classes are separated by $\bar{I}=.632$ (shaded regions $=C_{1}$, unshaded $=C_{2}$ ). As $\bar{I}(x)$ approaches $\bar{I}$, the split of the distribution of prototypes at $x$ into two classes becomes more pronounced (this is illustrated by Figure 4.5 where $x=5$ ).

The remainder of this section investigates the behavior of both deterministic and statistical pattern classification procedures when applied to this problem. In all cases the initial decision surface will be chosen at $\mathrm{L} / 2\left(\mathrm{w}_{1}=1.0, \mathrm{w}_{2}=-\mathrm{L} / 2\right)$. 

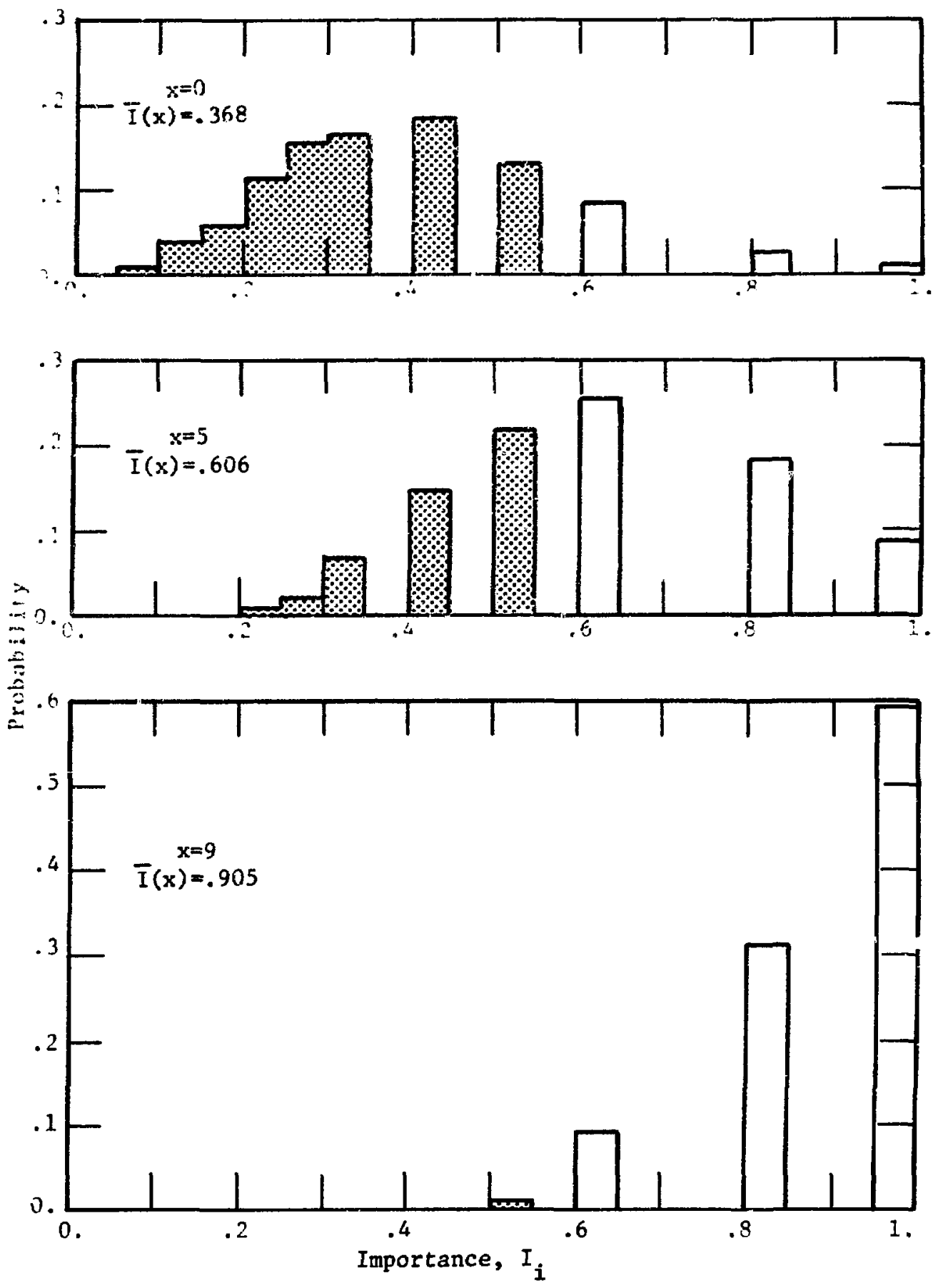

Figure 4.5 Distribution of Importances at $x=0,5$, and 9 for $L=10$ and $\bar{I}=.632$ ( 600 prototypes are sampled for each $x$ ) 


\subsection{1) Deterministic Techniques}

Figure 4.4 illustrates the fact that this problem does not produce linearly separable data since the $p\left(C_{i} \mid Y\right)$ are overlapping. Deterministic techniques will never converge to a single $g(Y)$ since there exists no $W$ which will correctly classify all Y's. However, if one is willing to use a $g(Y)$ which satisfies a large percentage of the Y's, then deterministic techniques can be used to find an appropriate $g(Y)$.

In the case of linearly inseparable data Fquat \pm n 3.6 cannot be used directly but must be replaced by the following

$$
\begin{aligned}
& \text { if } g\left(Y_{i}\right)>0 \text { and } Y_{i} \text { belongs to class } C_{1} \text {, } \\
& W^{\prime}=W-c\left(\frac{N_{2}}{N_{1}}\right) Y_{i}^{*} \text { or } \\
& \text { if } g\left(Y_{i}\right)<0 \text { and } Y_{i} \text { belongs to class } C_{2} \text {, } \\
& W^{\prime}=W+c Y^{*},
\end{aligned}
$$

where $N_{1}=$ number of prototypes in class $i$. 
Equation 4.4 prevents the class with the most prototypes from influencing the selection of $\mathrm{W}$ simply because it has more prototypes and not because it has a higher percentage of misclassified prototypes.

In this study the fractional correction rule will be used since it allows for a more controlled convergence when used with cverlapping distributions (i.e., the correction increment can be controlled through 1). Therefore, as given in Section 3.2.1, $c=\lambda|g| / Y * \cdot Y *$. The FORTRAN coding necessary to use the fractional correction rule with this problem is shown in Appendix D.

The results after 100 source particles for several slats of varying thickness and four values of $\lambda$ are summarized in Table 4.2. The behavior of the decision surface as a function of the number of source particles and a plot of weight space is shown in Figure 4.6 for a $\lambda$ of .5. As is seen from the data of Table 4.2 the value of $\lambda$ has only a small effect on the misclassification rate; however, it can greatly decrease the variability of the decision surface. This is useful since it is desirable to stop calculating the discriminant function once a suitable thas been found. Thus the variability can be used as an indicator of when to use the present discriminant function and stop adjustment of the weights.

The misclassification of prototypes after convergence is primarily due to the overlapping of the distributions shown in Figure 


\begin{tabular}{|c|c|c|c|c|c|c|}
\hline \multirow{2}{*}{$\begin{array}{c}\mathrm{L} \\
\text { Slab } \\
\text { Thickness } \\
\end{array}$} & \multirow[b]{2}{*}{$\begin{array}{l}\text { Learning } \\
\text { Parameter }\end{array}$} & \multicolumn{3}{|c|}{$\begin{array}{c}\text { Misclassification } \\
\text { Rate } \\
\end{array}$} & \multirow{2}{*}{$\begin{array}{c}\text { Mean } \\
\text { Decision } \\
\text { Surface } \\
\end{array}$} & \multirow[b]{2}{*}{ Variability } \\
\hline & & $\mathrm{C}_{1}$ & $\mathrm{C}_{2}$ & $\mathrm{C}_{1}+\mathrm{C}_{2}$ & & \\
\hline \multirow[t]{4}{*}{10} & $\lambda=.05$ & .357 & .181 & .259 & 3.57 & .128 \\
\hline & $\lambda=.2$ & .397 & .174 & .273 & 3.64 & .235 \\
\hline & $\lambda=.5$ & .371 & .177 & .263 & 4.02 & .364 \\
\hline & $\lambda=1.0$ & .348 & .181 & .255 & 4.63 & .446 \\
\hline \multirow[t]{4}{*}{50} & $\lambda=.05$ & .116 & .079 & .104 & 32.01 & .086 \\
\hline & $\lambda=.2$ & .103 & .077 & .094 & 32.76 & .105 \\
\hline & $\lambda=.5$ & .115 & .051 & .094 & 32.92 & .148 \\
\hline & $\lambda=1.0$ & .140 & .052 & .112 & 32.33 & .194 \\
\hline \multirow[t]{4}{*}{200} & $\lambda=.05$ & .043 & .034 & .041 & 167.4 & .048 \\
\hline & $\lambda=.2$ & .040 & .021 & .037 & 169.1 & .050 \\
\hline & $\lambda=.5$ & .057 & .026 & .052 & 166.7 & .082 \\
\hline & $\lambda=1.0$ & .089 & .026 & .080 & 162.7 & .125 \\
\hline
\end{tabular}

Table 4.2 Results of $\lambda$ and $L$ Variations After 100 Source Particles Using the Deterministic Classifier 


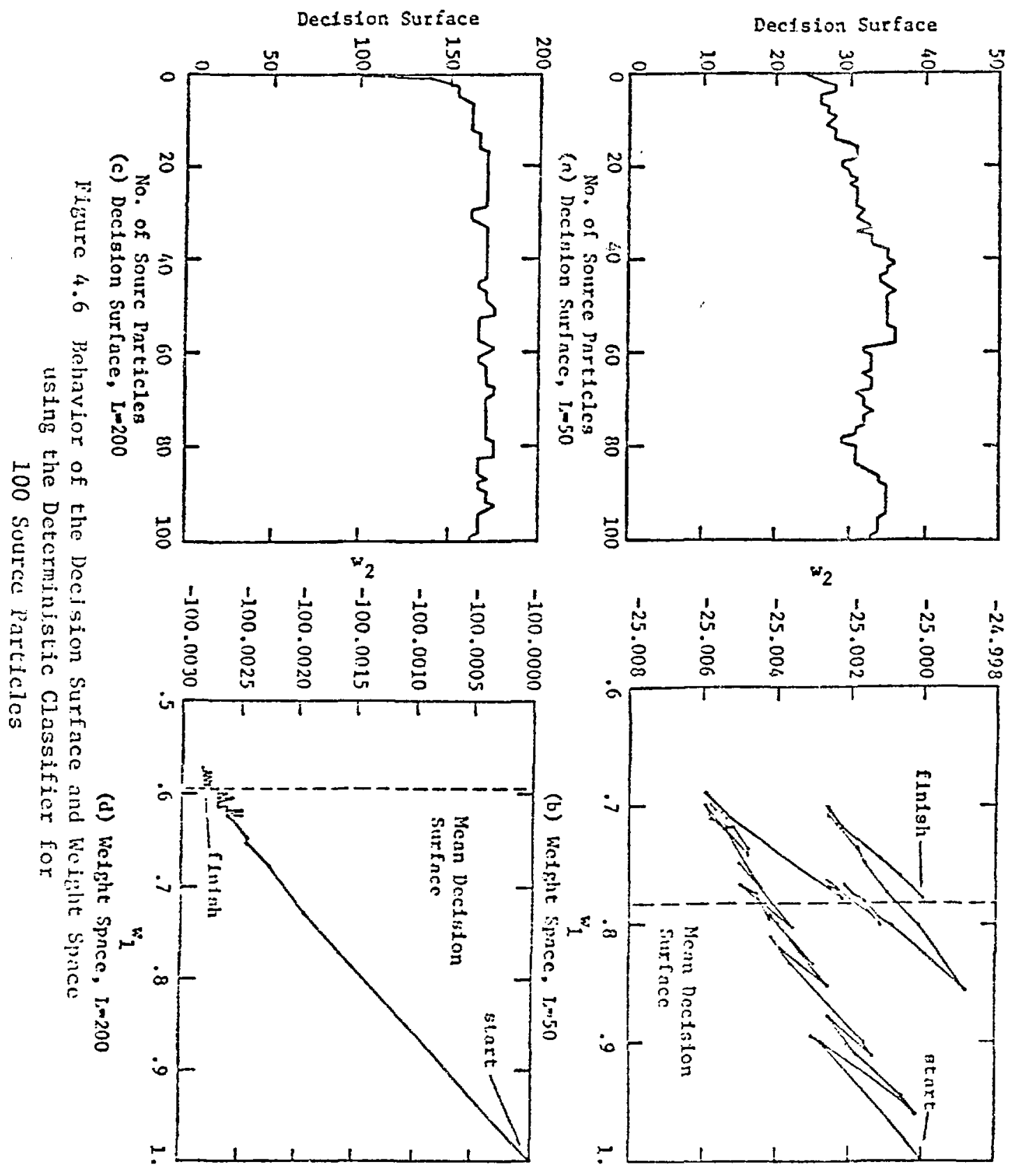


4.4. This overlapping is measured in terms of the error rate as given by

$$
\begin{aligned}
& \text { Error Rate }=\mathrm{F}_{\mathrm{r}}=\frac{100}{\mathrm{~L}}\left[\int_{0}^{\overline{\mathrm{x}}} \mathrm{p}\left(\mathrm{C}_{2} \mid \mathrm{Y}\right) \mathrm{d} \mathrm{x}+\int_{\overline{\mathrm{x}}}^{\mathrm{L}} \mathrm{p}\left(\mathrm{C}_{1} \mid \mathrm{Y}\right)\right] \\
& \text { where } \mathrm{F}_{\mathrm{r}}=\% \text { misclassification of prototypes due to overlapping } \\
& \text { of } \mathrm{P}\left(\mathrm{C}_{1} \mid \mathrm{Y}\right) \\
& \mathrm{L}= \text { slab thickness } \\
& \bar{x}= \text { the value of } x \text { for which } \mathrm{p}\left(\mathrm{C}_{1} \mid \mathrm{Y}\right)=\mathrm{p}\left(\mathrm{C}_{2} \mid \mathrm{Y}\right)
\end{aligned}
$$

Since the majority of misclassified prototypes come from an importance close to $\bar{I}$, much of the error can be eliminated by the introduction of a "buffer zone". A buffer zone consists of a band of importances from $I_{1}$ to $I_{2}\left(I_{1}<\bar{I}<I_{2}\right)$. Any track which has an importance $I$ such that $I_{1}<I<I_{2}$, is not placed in either class and $W$ is left unchanged. Results of using various buffer zones on the $P\left(C_{i} \mid Y\right)$ distributions are shown in Figure 4.7. The reduction in the overlapping area is given in Table 4.3 along with results obtained by using various buffer zones. As can be seen from this data, the buffer zones have the effect of lowering the misclassification rate considerably. The variability is lowered in some cases, but raised in others. This is due to the fact that the majority of variability is caused by prototypes outside the buffer 


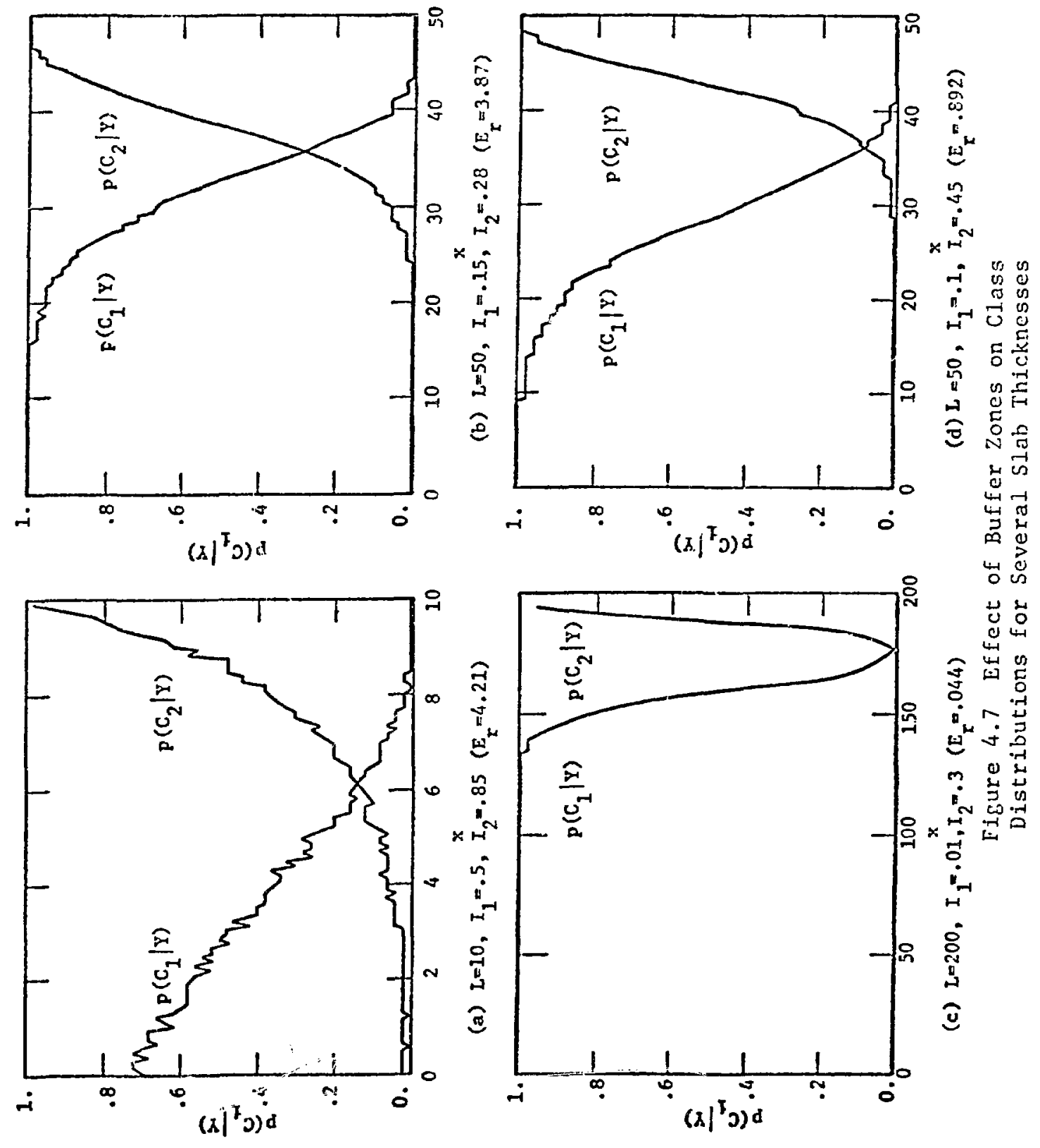




\begin{tabular}{|c|c|c|c|c|}
\hline $\begin{array}{c}\text { Buffer } \\
\text { Zone } \\
\end{array}$ & $\mathrm{E}_{\mathrm{r}}$ & $\begin{array}{c}\text { Misclassification } \\
\text { Rate } \\
\end{array}$ & $\begin{array}{c}\text { Mean } \\
\text { Decision } \\
\text { Surface } \\
\end{array}$ & Variabiiity \\
\hline \multicolumn{5}{|l|}{$L=10$} \\
\hline none & 23.0 & .259 & 3.572 & .128 \\
\hline $.6-.75$ & 14.1 & .240 & 3.929 & .089 \\
\hline $.5-.85$ & 4.2 & .153 & 4.532 & .056 \\
\hline \multicolumn{5}{|l|}{$L=50$} \\
\hline none & 8.85 & .104 & 32.01 & .086 \\
\hline $.18-.22$ & 6.72 & .086 & 32.97 & .085 \\
\hline $.15-.28$ & 3.87 & .064 & 33.04 & .089 \\
\hline $.10-.45$ & .89 & .045 & 31.80 & .101 \\
\hline \multicolumn{5}{|l|}{$\mathrm{L}=200$} \\
\hline none & 2.96 & .041 & 167.4 & .048 \\
\hline $.025-.12$ & .64 & .024 & 167.0 & .054 \\
\hline $.010-.30$ & .04 & .024 & 161.3 & .059 \\
\hline
\end{tabular}

Table 4.3 Effect of Buffer Zones on the Performance of the Deterministic Classifier after 100 Source Particles 
zone. These misclassifications thus have more effect since there are fewer prototypes* in the buffer zone runs.

In conclusion, deterministic techniques work satisfactorily if $\lambda$ is kept small $(\lambda=.05$ is sufficient) and buffer zones are used. One interesting characteristic is that the larger $I$, the better the classifier performs. This is quite favorable since it is for large $L$ that one needs variance reduction the most.

\subsection{2) Statistical Techniques}

In Section 3.3 the statistical approach to pattern recognition was described in which weight adjustments were made based on the average behavior of the prototypes. In this section Fquations 3.20 and 3.21 are used to adjust the weights for several different 10 ss functions, $S\left(W, Y, C_{i} \mid C_{k}\right)$. Table 4.4 1ists the loss functions which are investigated and thetr corresponding $\nabla S$ components. A description of these loss functions and the derfvation of the $\nabla S$ components are given in Appendix E. From Equation 3.20 the weight vector, $W$, is incremented according to

\footnotetext{
*Feature vectors falling within the buffer zone are not counted as prototypes since their classification is not deternined.
} 


$$
\begin{aligned}
& S\left(W, Y, C_{1} \mid C_{j}\right) \\
& \frac{\partial S\left(W, Y, C_{2} \mid C_{1}\right) *}{\partial w_{1}} \\
& \mathrm{~d}=\frac{|\mathrm{g}|}{\mathrm{w}_{1}} \\
& -\frac{w_{2}}{w_{1}^{2}} \\
& d=\sqrt{\frac{|g|}{w_{1}}} \\
& -\frac{w_{2}}{w_{1}^{2}}\left(\frac{1}{2} \sqrt{\left|\frac{w}{g}\right|}\right) \\
& \mathrm{d}^{2}=\frac{\mathrm{g}^{2}}{\mathrm{w}_{1}^{2}} \\
& D=\frac{|g|}{\sqrt{1+y^{2}}} \\
& -\frac{w_{2}}{w_{1}^{2}}\left(\frac{2|g|}{w_{1}}\right) \\
& \frac{1}{w_{1}}\left(\frac{2|g|}{w_{1}}\right) \\
& \frac{y}{\sqrt{1+y^{2}}} \\
& \frac{1}{w_{1}} \\
& \frac{\partial S\left(\mathrm{~W}, \mathrm{Y}, \mathrm{C}_{2} \mid \mathrm{C}_{1}\right) *}{\partial \mathrm{w}_{2}} \\
& \frac{1}{w_{1}}\left(\frac{1}{2} \sqrt{\left|\frac{w_{1}}{g}\right|}\right) \\
& \frac{1}{\sqrt{1+y^{2}}}
\end{aligned}
$$

*For all loss functions

$$
\begin{aligned}
& \frac{\partial S\left(W, Y, C_{2} \mid C_{1}\right)}{\partial w_{1}}=\frac{-\partial S\left(W, Y, C_{1} \mid C_{2}\right)}{\partial w_{1}} \\
& \frac{\partial S\left(W, Y, c_{2} \mid C_{1}\right)}{\partial w_{2}}=\frac{-\partial S\left(W, Y, C_{1} \mid C_{2}\right)}{\partial w_{2}}
\end{aligned}
$$

Table 4.4 Loss Functions and IS Components 


$$
w_{i+1}=w_{1}-\nabla \tilde{R}(w)=\left[\begin{array}{c}
w_{1} \\
w_{2}
\end{array}\right]_{i+1}=\left[\begin{array}{c}
w_{1} \\
w_{2}
\end{array}\right]_{i}-\lambda\left[\begin{array}{c}
\frac{\partial \tilde{R}}{\partial w_{1}} \\
\frac{\partial \tilde{R}}{\partial w_{2}}
\end{array}\right]
$$

Appendix $F$ contains a listing of the FORTRAN code used to implement Equation 4.6. The risk as defined by Fquation 3.10 is given by

$$
\begin{aligned}
& \tilde{R}(H)=\widetilde{R}\left(\mathrm{H}, \mathrm{C}_{1}\right)+\widetilde{R}\left(\mathrm{H}, \mathrm{C}_{2}\right) \\
& =\frac{\sum_{n=1}^{N} s\left(H, Y_{n}^{1}, C_{2} \mid C_{1}\right)}{M_{1}}+\frac{\sum_{n=1}^{N} s\left(H, Y_{n}^{2}, C_{1} \mid C_{2}\right)}{M_{2}}
\end{aligned}
$$

where: $\mathrm{N}_{i}=$ total number of misclassified prototypes in class $\mathrm{C}_{1}$

$M_{i}=$ total number of prototypes in class $C_{i}$ $\mathrm{Y}_{\mathrm{n}}^{1}=$ the $\mathrm{n}^{\prime}$ th misclassified feature vector of class $C_{1}$ $S\left(W, Y_{n}^{j}, C_{1} \mid C_{j}\right)=$ the loss incurred when feature vector $\mathrm{Y}_{\mathrm{n}}^{j}$ is misclassified into class $\mathrm{C}_{1}$.

The loss $S$ is evaluated using the value of that exists at the time $Y_{n}^{j}$ is misclassifled. 
By using Equation 4.7 and the $S\left(W, Y, C_{i} \mid C_{j}\right)$ given in Table 4.4, $K(W)$ can be evaluated for different values of $W$. This was done for the prototypes created by the slab Monte Carlo problem of Figure 4.2 (with $\mathrm{L}=10$ and $\bar{I}=$ mean importance $=.632$ ) using 12 different values of a fixed decision surface (decision surface location $=-w_{2} / \mathrm{w}_{1}=$ constant $=1,3,3.5,4,4.5,5,5.5,6,7,8,9,10)$ for each loss function. For these calculations $w_{1}=1$ resulting in $-w_{2}$ being equal to the above decision surface locations. Each estimate of $\tilde{R}(W)$ was determined by using 200 source particles (1024 prototypes). The resulting 12 values of $\tilde{\mathrm{R}}(\mathrm{V})$ for each loss function were then fitted ${ }^{37}$ with a second order polynomial. The resulting curves are illustrated in Figure 4.8 for the loss functions of Table 4.4 as well as the loss function

$$
S\left(W, Y, C_{i} \mid C_{j}\right)=\text { constant }
$$

Each curve has been normalized to its minimum $r i s k, \tilde{k}_{\min }(W)$, resulting in a relative loss function. The location of the deciston surfaces for minimum risk, $x_{\text {min }}$ as determined by the fitted polynomials are: 


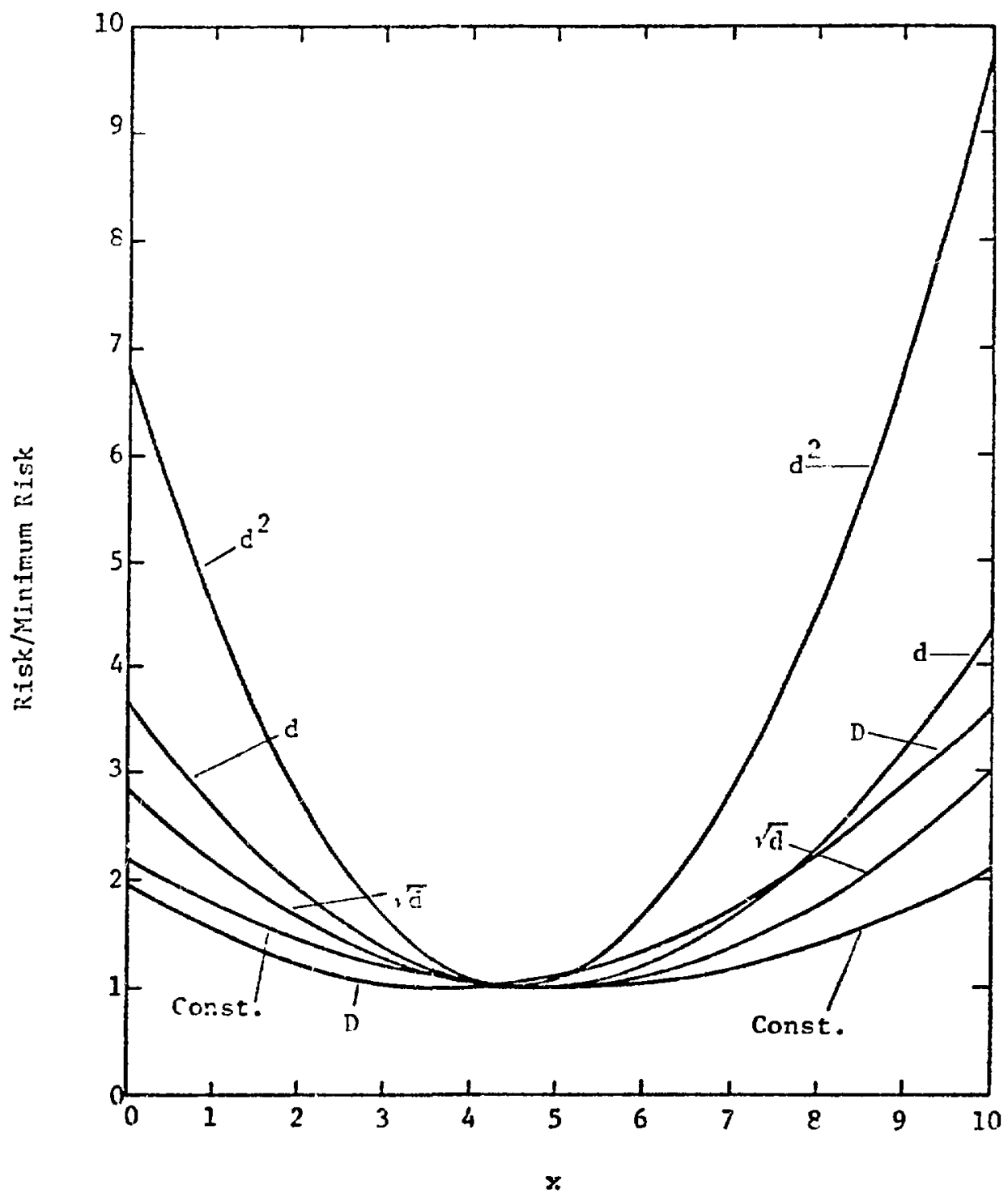

Figure 4.8 Relative Risi as a Function of a Decision Surface location for Various loss Functious 
Loss Function

Constant

$\sqrt{d}$

d

$d^{2}$

D
Decision Surface Location

for Minimum Risk ( $\mathrm{min}_{\text {min }}$ )

Error*

5.11

4.50

.074

4.73

4.89

.104

3.78

*This estimated error is for the $1 \sigma$ level and includes on $1 y$ the error introduced by the least squares curve fitcing procedure ${ }^{37}$.

Equation 4.7 is equivalent to the following:

$$
\begin{array}{ll}
\text { Risk }=R_{1}+R_{2} & \text { for loss }=\text { Constant } \\
\text { Risk }=R_{1} \sqrt{d_{1}}+R_{2} \sqrt{d_{2}} & \text { for loss }=\sqrt{d} \\
\text { Risk }=R_{1} \bar{d}_{1}+R_{2} \bar{d}_{2} & \text { for loss }=d \\
\text { Risk }=R_{1}\left(\overline{d_{1}^{2}}\right)+R_{2}\left(\overline{d_{2}^{2}}\right) & \text { for loss }=d^{2} \\
\text { Risk }=R_{1} \bar{D}_{1}+R_{2} \bar{D}_{2} & \text { for loss }=D
\end{array}
$$

where $R_{i}=$ misclassification rate for $C_{i}=N_{i} / M_{i}$

$$
N_{i}=\text { no. of misclassified prototypes for } C_{i}
$$




$$
\begin{aligned}
M_{i} & =\text { total no. of prototypes for } C_{i} \\
\bar{d}_{i} & =\text { average } d \text { of misclassified prototypes of } c_{i} \\
\sqrt{d_{i}} & =\text { average } \sqrt{d} \text { of misclassified prototypes of } C_{i} \\
\left(\overline{d_{i}^{2}}\right) & =\text { average } d^{2} \text { of misclassified prototypes of } C_{i} \\
D_{i} & =\text { average } D \text { of misclassified prototypes of } C_{i} \\
i & =1,2
\end{aligned}
$$

The decision surface location for which

$$
p\left(C_{1} \mid Y\right)=p\left(C_{2} \mid Y\right)
$$

Ininimizes the total misclassification rate as given by

$$
R_{T}=\frac{N_{1}+N_{2}}{M_{1}+M_{2}}
$$

but does not minimize the risk as given by

$$
\text { Risk }=R_{1}+R_{2}=\frac{N_{1}}{M_{1}}+\frac{N_{2}}{M_{2}}
$$


unless the median is used for $\bar{I}$ (i.e., $M_{1}=M_{2}$ ). Therefore, the minimum risk location for loss $=$ constant is not the same as the $p\left(C_{1} \mid Y\right)=p\left(C_{2} \mid Y\right)$ location of Figure 4.4a. The other loss functions further affect the optimum Iocation by introducing the average value of a function of $d$ or D. Of these, the risk using loss $=\mathrm{D}$ has the smallest $\mathrm{x}_{\mathrm{min}}$. This is because the $y_{1}(=x)$ in the denominator of the expression for D (See Table 4.4) causes misclassified prototypes with small $\mathrm{x}$ to be weighed more heavily than those with large $x$. This causes the optimum decision surface to move so as to decrease $g(Y)$ for smaller $x$ (i.e., it moves to a smaller $\left.x_{m i n}\right)$. As $L$ becomes large, the $p\left(C_{i} \mid Y\right)$ become more symmetric and (when the mean importance is used for $\overline{\mathrm{I}})$ the $\mathrm{p}\left(\mathrm{C}_{1} \mid \mathrm{Y}\right)=\mathrm{p}\left(\mathrm{C}_{2} \mid \mathrm{Y}\right)$ 1ocation increases (see Figure 4.4). These two effects cause the $x_{\min }$ of different loss functions to approach the same value. It was found that for the case where $L=200$, the differences in $x_{\min }$ are indistinguishable as far as the classifier is concerned.

For the general loss function given by

$$
\text { Loss }=\mathrm{d}^{\mathrm{k}}
$$

as $k \rightarrow \infty$ the maximum of the misclassification distances are minimized and as $k \rightarrow 0$ the percentage of misclassifled patterns is minimized. This 
is illustrated in Figure 4.8 by the high ratio of $\widetilde{R}(W) / \widetilde{R} \min (W)$ for loss $=d^{2}$ and the lower ratio for loss $=\sqrt{d}$.

The misclassification rate and variability of the decision surface are shown in Figure 4.9 and 4.10 respectively for the different loss functions over a range of $\lambda$. These plots are for the problem described earlier in Sectiun 4.2, using a slab thickness of 200 and a source of 1000 particles. For all loss functions, the final value of the decision surface was between $x=166$ and $x=167$. These tests 111ustrate two important phenomena: (1) there is not a great amount of difference between the performance of the different loss functions after 1000 particles (i.e., when using optimum values of $\lambda$ for each) and (2) the performance of the pattern classifier is dependent on the value of $\lambda$. For each loss function there is a range of $\lambda$ (approximately three decades wide) over which the performance is relatively constant. A $\lambda$ below this range leads to decreased performance because of the increase in convergence time it requires. A $\lambda$ above this range leads to poor performance since it overcompensates for the correction.

The misclassification rate as a function of source particles started is shown in Figure 4.11. The values of $\lambda$ used in these runs were chosen from Figures 4.9 and 4.10 so as to optimize performance and are 


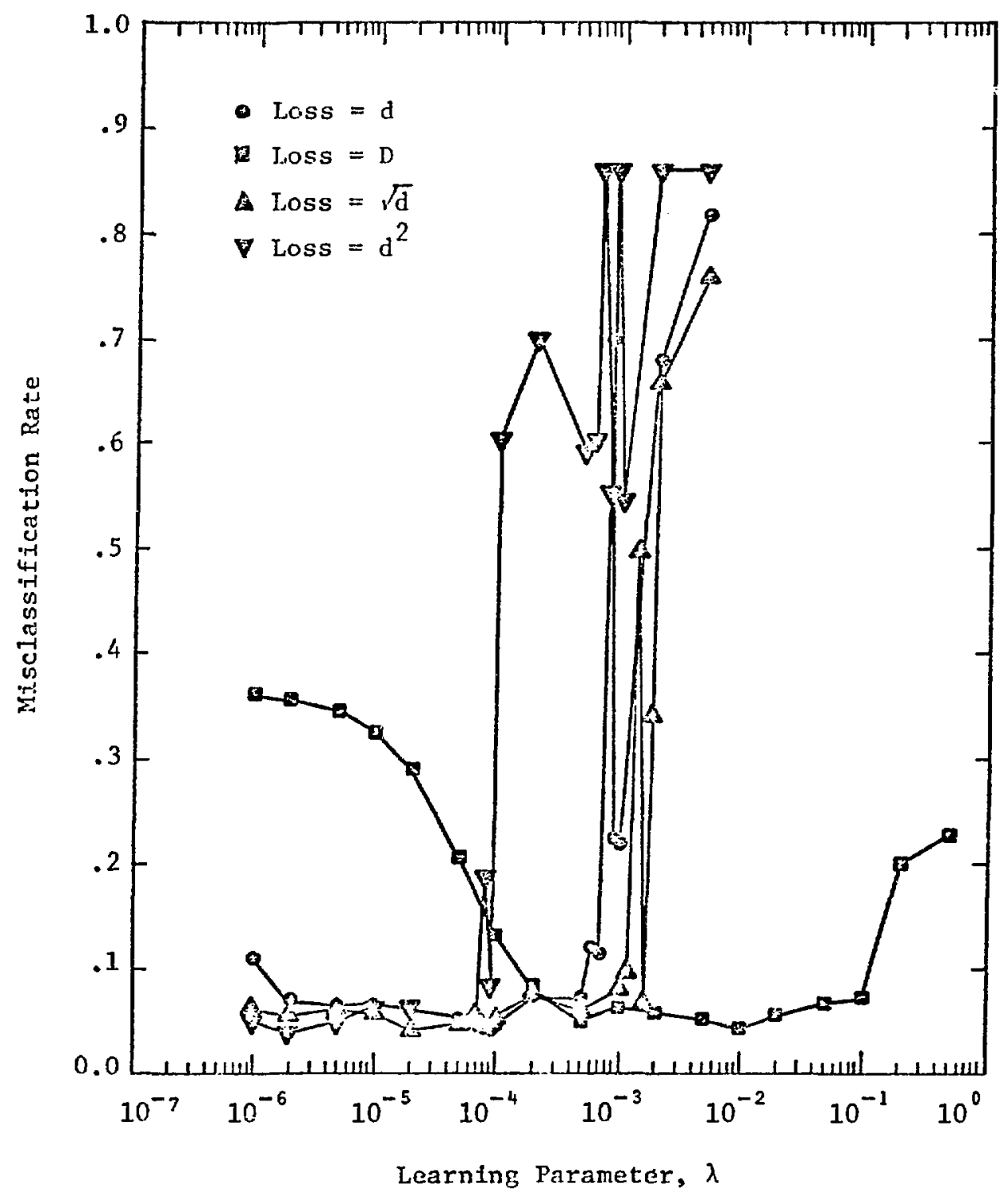

Figure 4.9 Misclassification Rate Vs. $\lambda$ for Various Loss Functions 


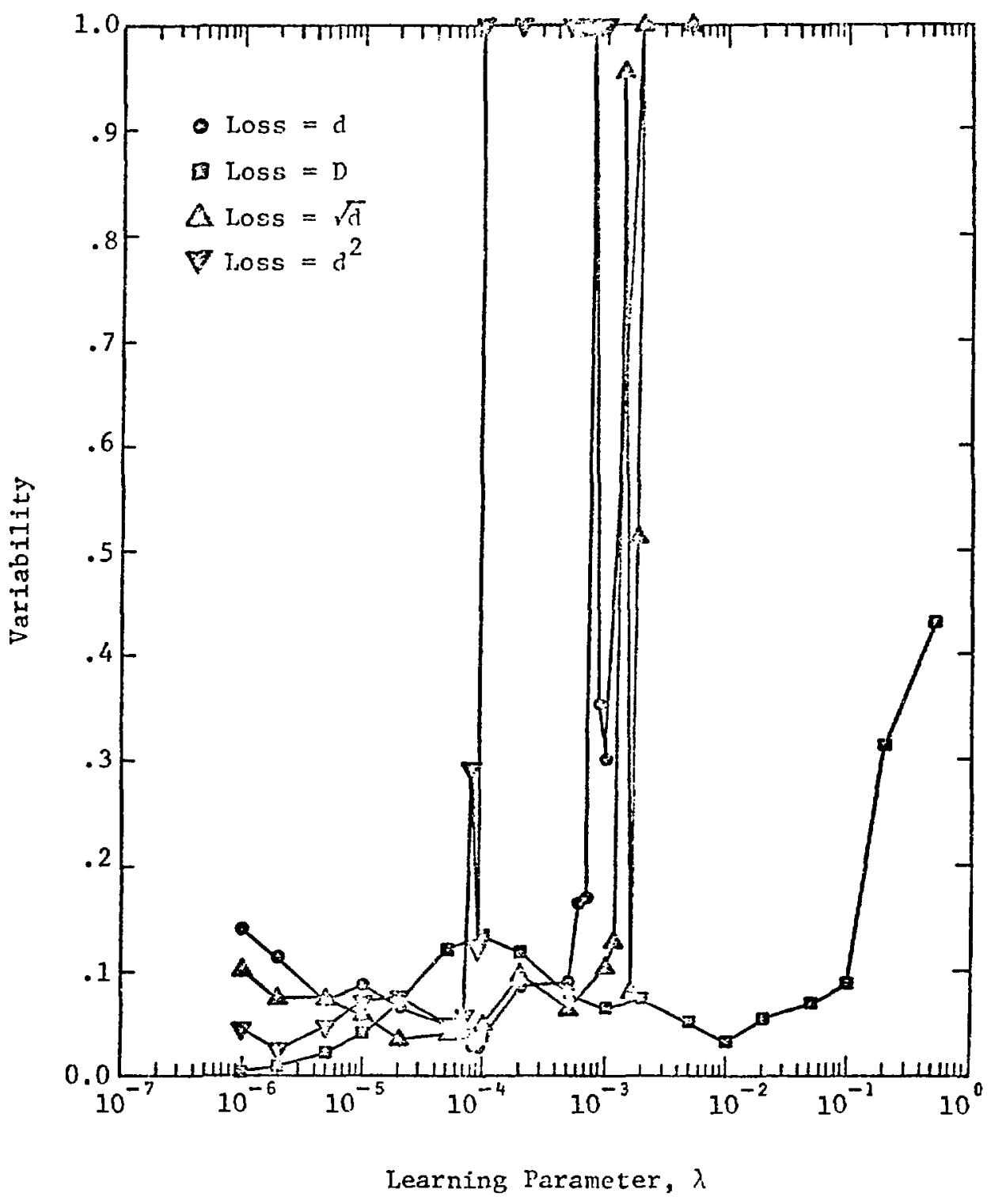

Figure 4.10 Variability Vs. $\lambda$ for Various Loss Functions 


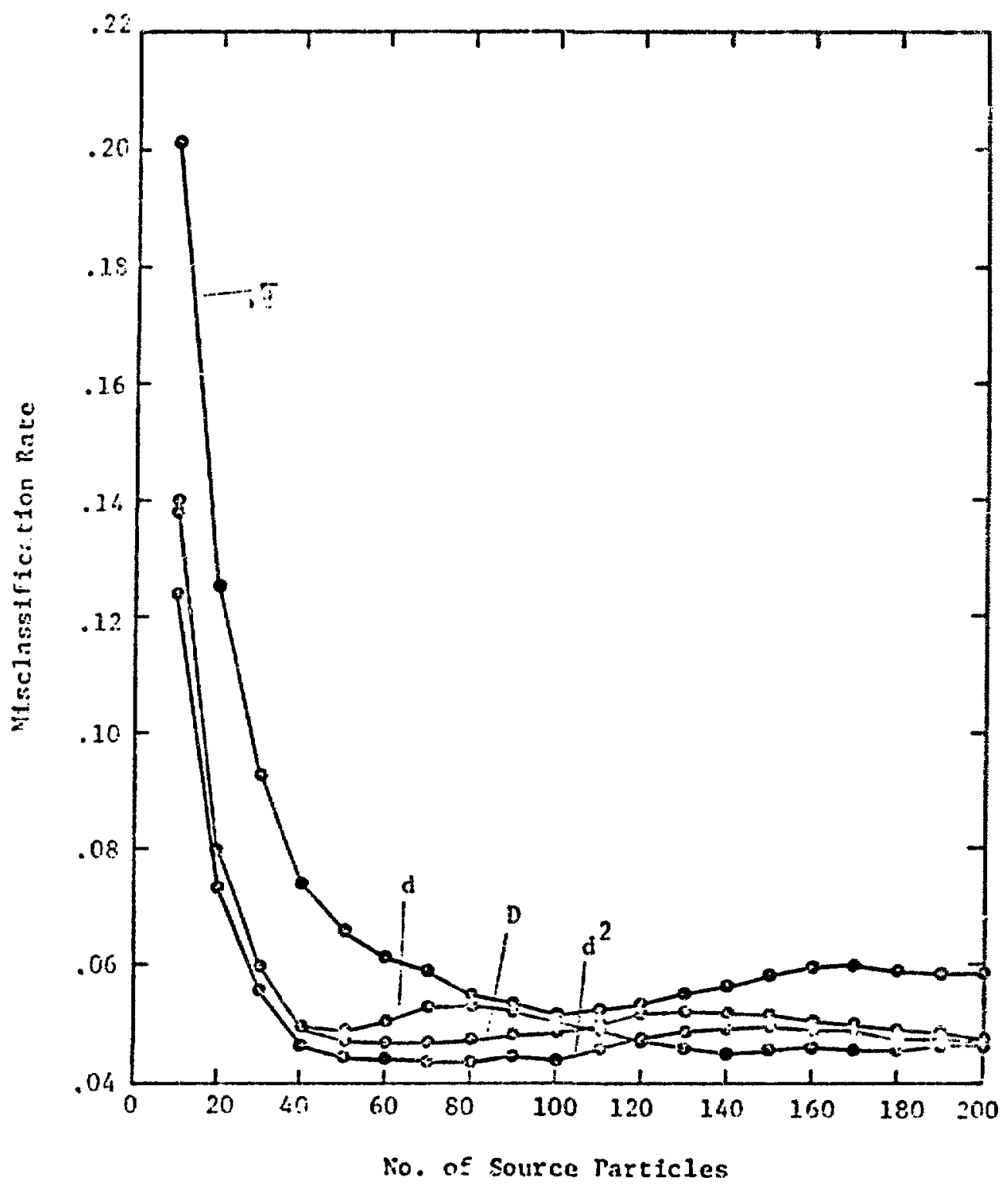

Figure 4.11 Uisclassification Race Vs. Number of Source Particles for various Loss functions 


\begin{tabular}{|c|c|}
\hline Loss Function & 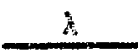 \\
\hline$\sqrt{d}$ & .00002 \\
\hline a & . orone \\
\hline$t^{2}$ & $.0 n n n 02$ \\
\hline 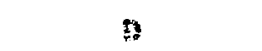 & .0 \\
\hline
\end{tabular}

With the exeeption of loss -14 , all zoss functions load to converience

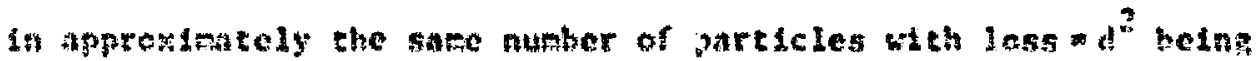

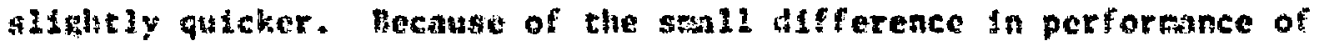
the shove lows functions, loss ad appears to be the nose actuactive pecatse of 1 ss computational simplictey. In all cases the statistical appronch resutes in osclilations about the cptinum declston surface. Altrough the cechntque is guaranteed to converge. ${ }^{31}$ runs ande wh as many as 20,000 source partleles still show the presence of this oscillation although it does decrease in amplitude.

To alloviace this osclliation, buffer zones are introduced. The effect of using buffer zones on the risk is illustrated in Figures $4.12 \mathrm{a}$ and $4.12 \mathrm{~b}$. Figure $4.12 \mathrm{a}$ indicates that buffer zones have the same effect as Increasing $k$ in Equation 4.8; however, Figure 4.12b shows that Jlthough the relative risk is increased, the absolute risk is actually decteased. Figure 4.13 shows the effect of the buffer zones on the misclassification rate and the varlability of the decision surface. Although the varlability is less for the buffer zone runs 
Risk/Minimum lisk

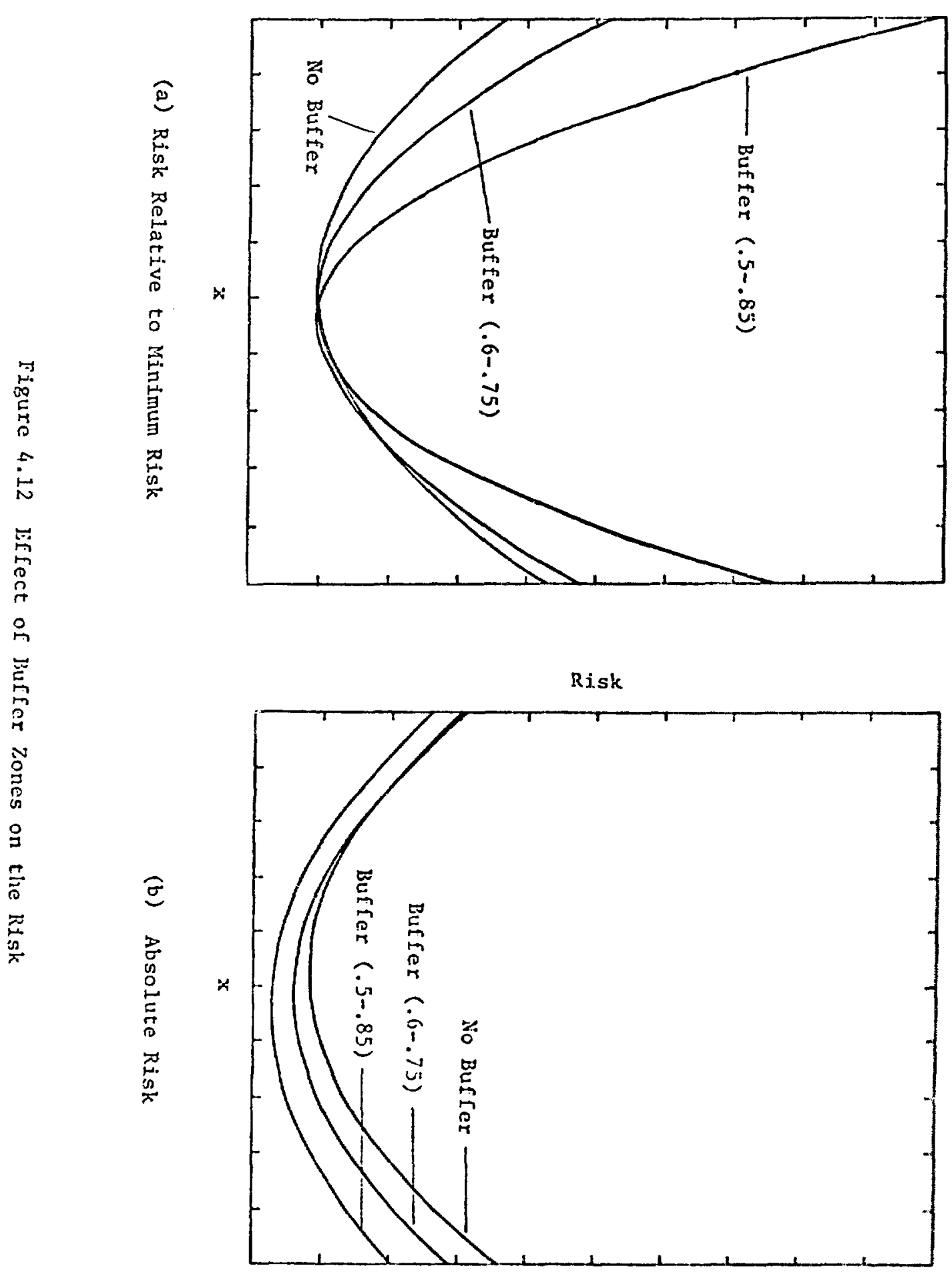




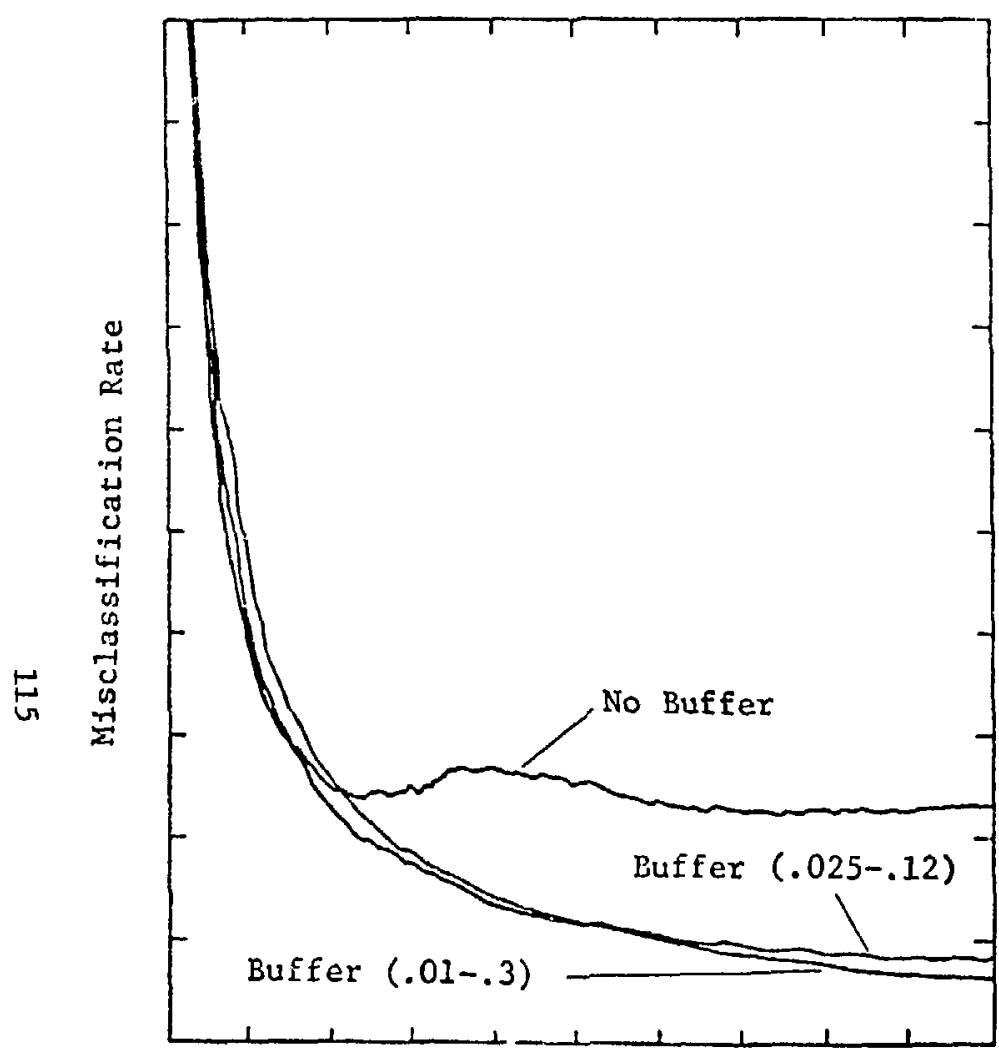

No. of Source Particles

(a) Misclassif1cation Rate

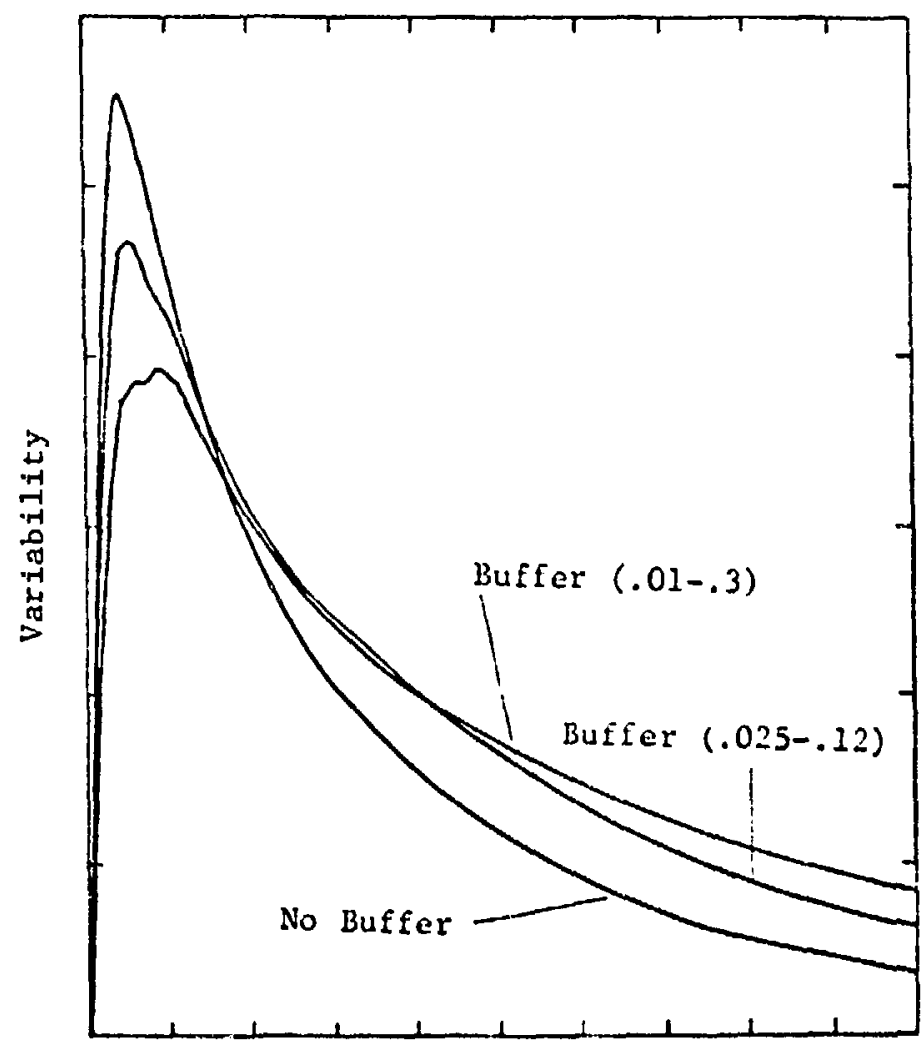

No. of Source Particies

(b) Variabilicy

Figure 4.13 Effect of Buffer Zones on Misclassification Rate and Variability 
curing the Initial approach to the decizion surface, after tisis approach the varlability of the non-buffer gone ratis less. This behavior is consistent with the deterministic buffer runs of section 4.2 .1 and indicates that although the $y_{i}$ that lie in the buffer zone are not classified, they should be counted as prototypes when calculating the variability in order to obtain a true measure of the convergence. The smoothing of the convergence due to huffer zones is illustrated by Figure 4.14 for the problen used earlier in this section.

In summary, the statistical approach produced satisfactory results which do not depend greatly upon the selection of the loss function. A loss function proportional to the misclassification distance appear 5 to be the most attractive since it requires fewer computations. Although the statistical approach does converge with time, it appears that like the deterministic approach, a decision surface will have to be selected prior to a final convergence. The use of buffer zones reduces the error after the initial approach and the varlability (as calculated) during the inftlal approach. The increase in vartability after the decision surface has begun to converge, when buffer zones are used, can be reduced by treating buffer zone feature vectors as prototypes when calculating the variability. Buffer zones are also attractive since by reducing the number of weight adjustments, they reduce the amount of computer time used. 


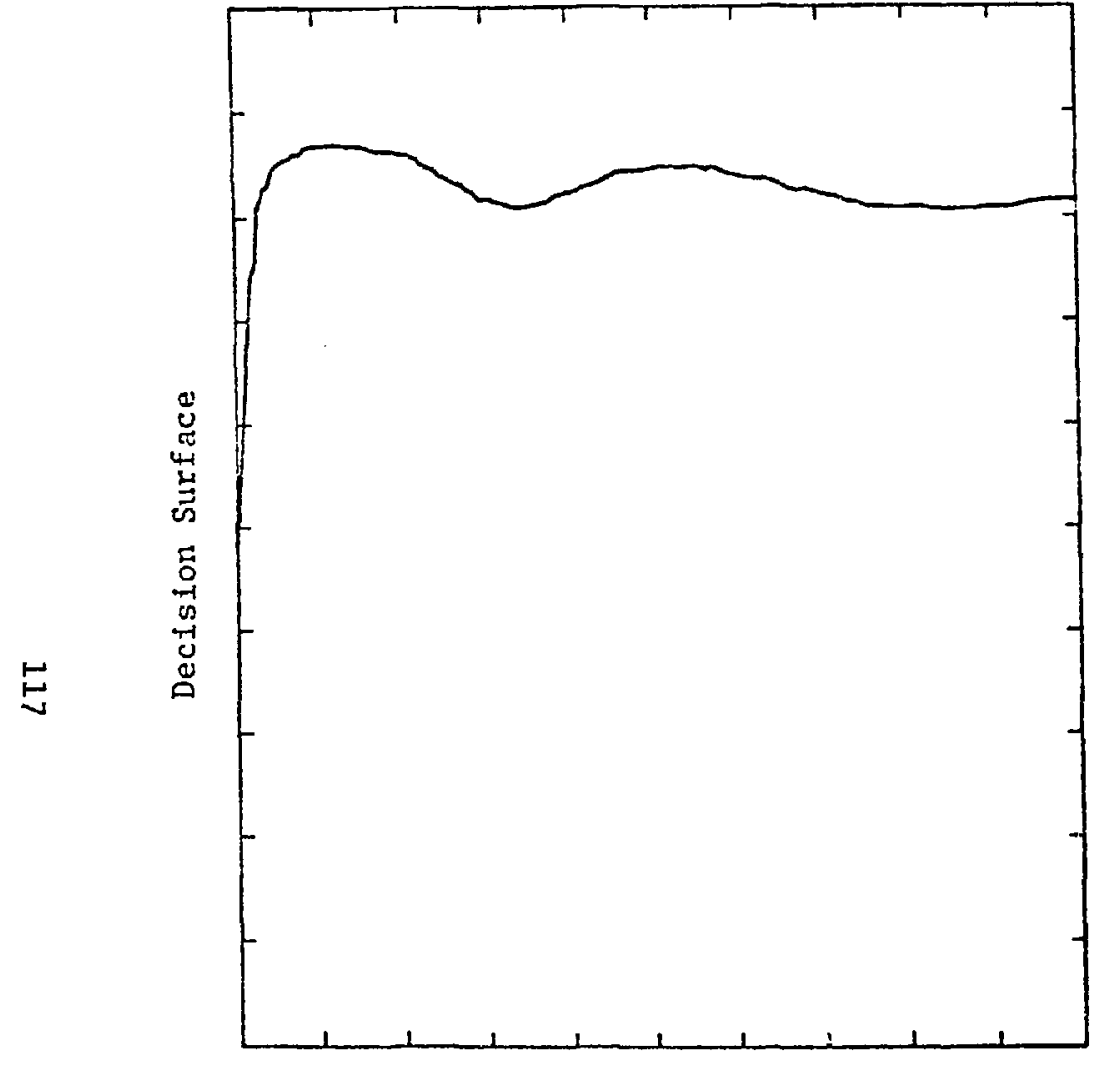

No. of Source Particles

(a) No Buffer

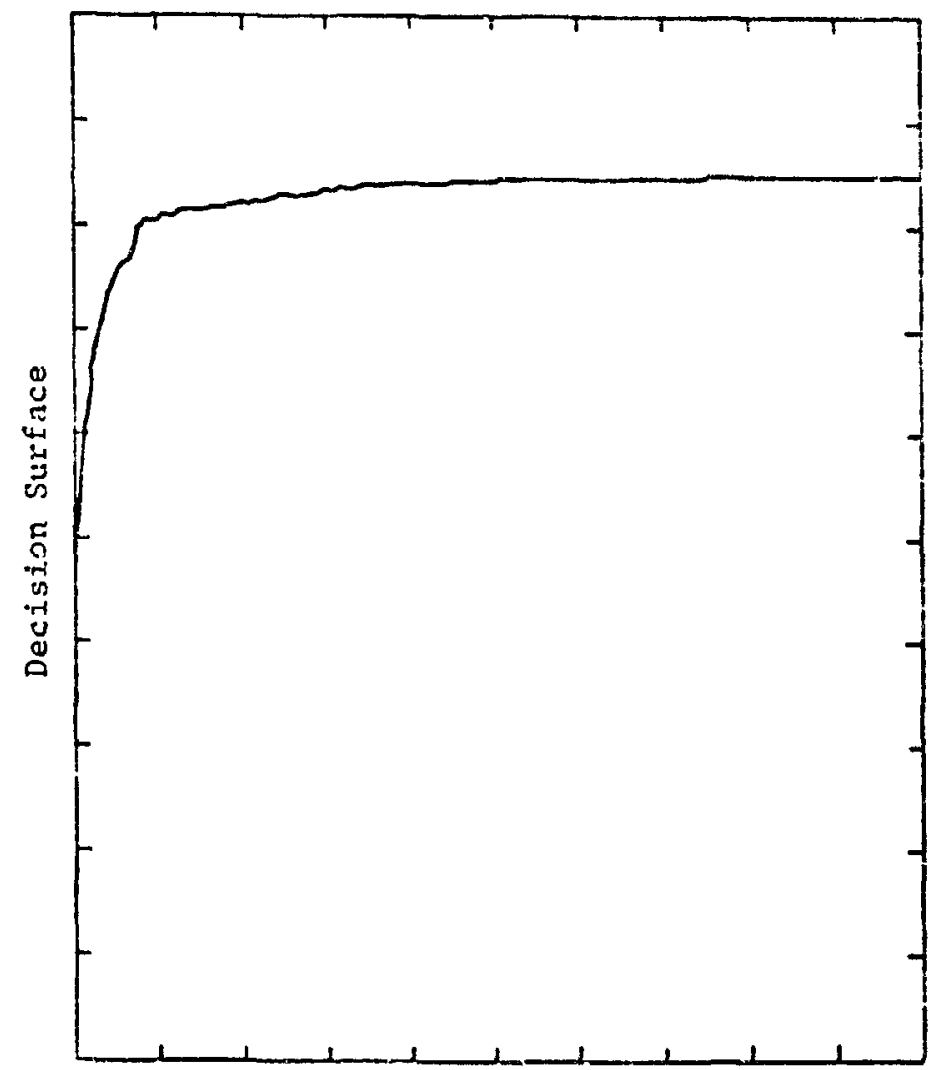

No. of Source Parcicles

(b) Buffer $(.01-.3)$

Figure 4.14 Smoothing of the Convergence Due to Buffer Zones 


\subsection{3) Comparison of Deterministic and Statistical Techniques}

In the previous two sections statistical and deterministic techniques have been implemented on different thickness slabs using a range of learning parameters $(\lambda)$ and different buffer zones. Fimure 4.15 compares deterministic and statistical classifiers using $L=200$, $\bar{T}_{t}=.5, \Sigma_{s}=.4$, no buffer zones, and an $\bar{I}=.05$. Both runs use an optimum $\lambda$ for the specific technique $(\lambda=.05$ for deterministic and $\lambda=.00008$ for statistical). Although the deterministic classifier leads to slightly fewer misclassifications in the early stages of the run, as the decision surface begins to converge (after about 40 particles) the two techniques are very competitive.

The variability of the decision surface appears to be considerably lower for the deterministic case. The reason for this can be seen from Figure 4.16 in which the average decision surface and the decision surface are plotted as a function of particles started. In the deterministic case a single prototype can cause the decision surface to be altered in a given direction. However, for the statistical classifier, the decision surface is altered in the difection indicated by the average properties of the prototypes. This behavior leads to a buildup of misclassification in the other direction. Although the variability of

the decision surface does appear greater for the statistical classifier during the early stages, the final variability of the deterministic classifier will never go to zero; whereas for the statistical, it is guaranteed. 


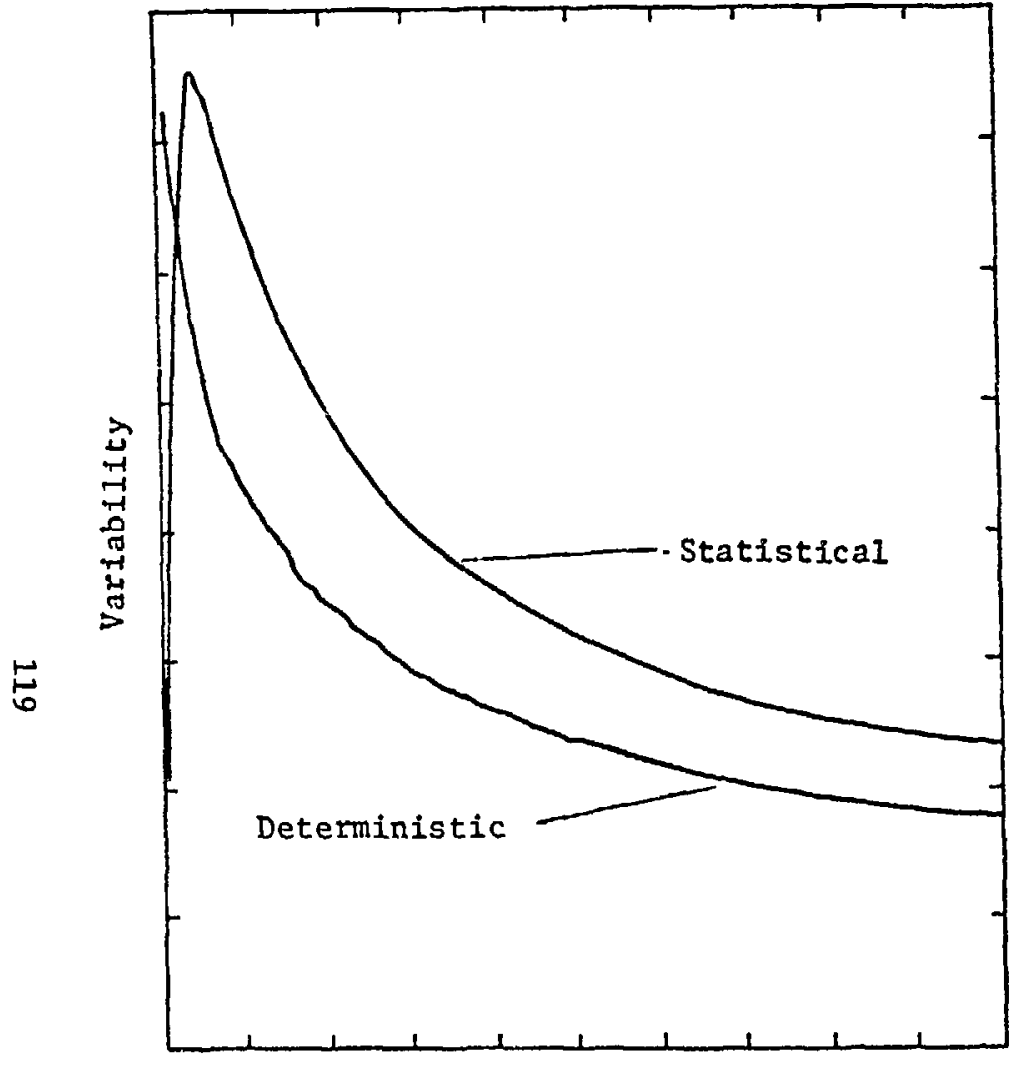

No. of Source Particles

(a) Variability

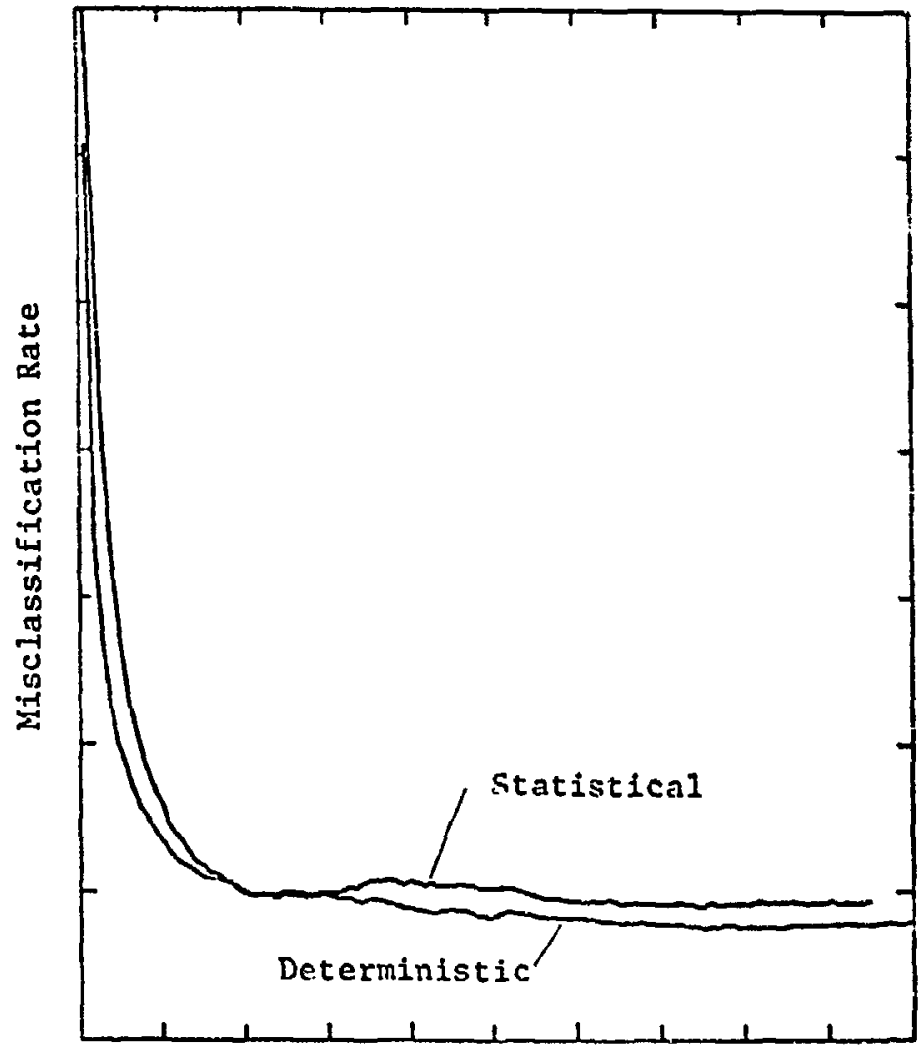

No. of Source Farticles

(b) Misclassification Rate

Figure 4.15 Comparison of the Performance of Deterministic and Statistical Classifiers 


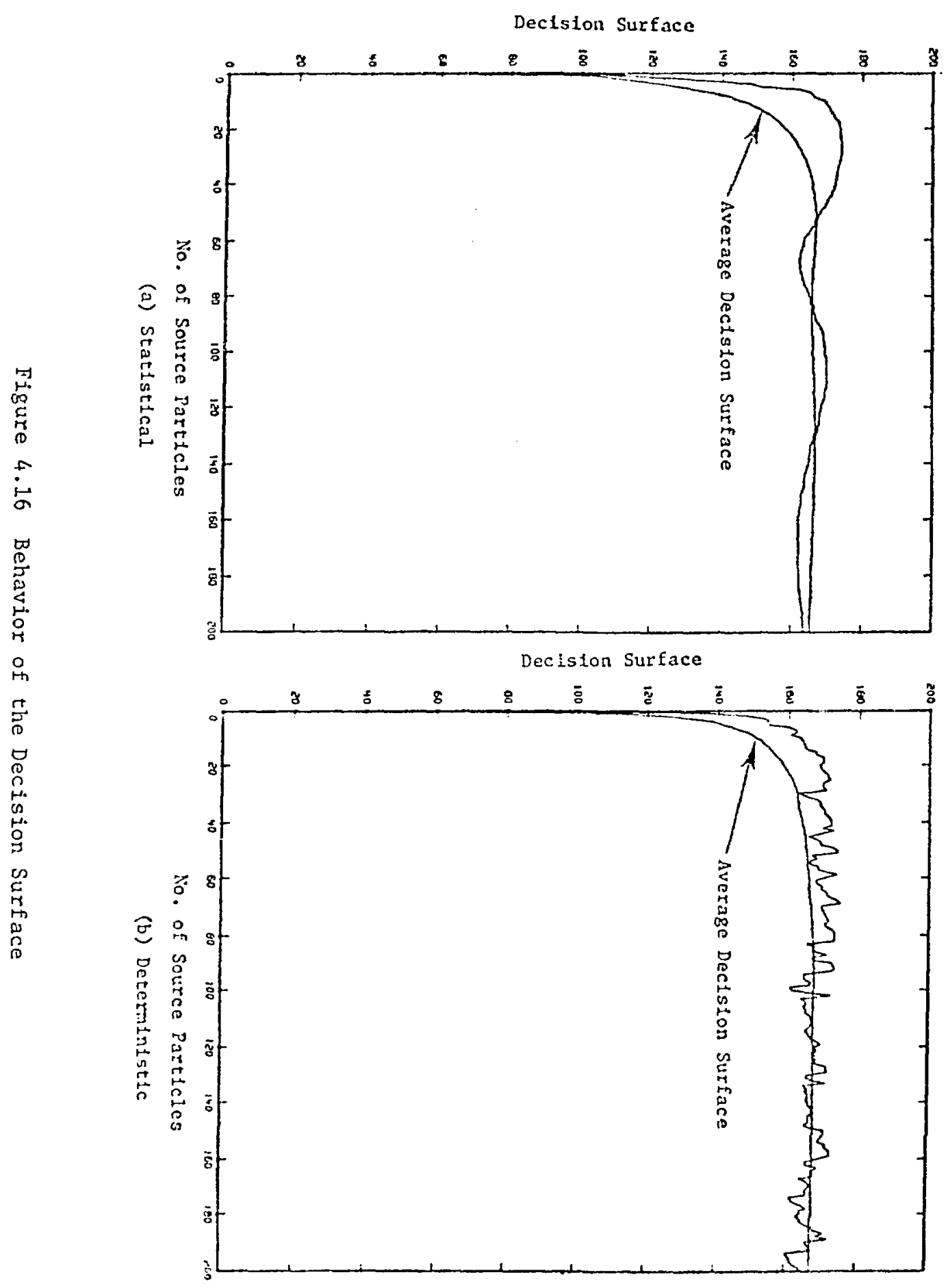


The computer time spent for the two techniques can be compared by using Equation 4.9

$$
\begin{aligned}
& T=N\left(t_{A}+t_{L} f_{L}+t_{C}{ }_{L} f_{C}\right), \\
& \text { where } T=\text { total computer time spent for pattern recognition, } \\
& N=\text { total number of prototypes produced, } \\
& t_{A}=\text { time required to determine the classification of a } \\
& \text { prototype, } \\
& t_{L}=\text { time required to determine if a prototype is mis- } \\
& \text { classified, } \\
& { }_{\mathrm{C}}=\text { time required to adjust weights, } \\
& f_{C}=\text { fraction of classifiable prototypes which are mis- } \\
& \text { classified, and } \\
& f_{L}=\text { fraction of prototypes which are classifiable (1ie } \\
& \text { outside the buffer zone). }
\end{aligned}
$$

Using suitable timing schemes results in the values shown in Table 4.5 . The following results are obtained for $\mathrm{T}$ after 40 particles are started (resulting in $N=4013$ ) with no buffer zone (i.e., $f_{L}=1.0$ ).

Statistica1 $\left(\mathrm{f}_{\mathrm{C}}=.050\right)$

$$
\mathrm{T}=1.65 \times 10^{-2}+.40 \times 10^{-2}+.21 \times 10^{-2}=2.26 \times 10^{-2}
$$




\section{Statistical}

Deterministic

$\begin{array}{lll}t_{C} & 10.5 \times 10^{-6} & 3.5 \times 10^{-6} \\ t_{L} & 1.0 \times 10^{-6} & 1.0 \times 10^{-6} \\ t_{A} & 4.1 \times 10^{-6} & 4.1 \times 10^{-6}\end{array}$

Table 4.5 Timing Parameters for the One Dimensional Problem

Deterministic $\left(\mathrm{E}_{\mathrm{C}}=.051\right)$

$$
\mathrm{T}=1.65 \times 10^{-2}+.40 \times 10^{-2}+.07 \times 10^{-2}=2.12 \times 10^{-2}
$$

From these results it is seen that the two techniques are comparable since the majority of time spent is not for weight adjustment but for class identification. However, since the variability is less for the deterministic classifier, the statistical classifier requires more prototypes (greater $N$ ) to get the same results. Thus the deterministic classifier appears to be the most advantageous with respect to time. The above times can be reduced by the use of buffer zones as shown below for a buffer zone of .01 to $.30\left(f_{L}=.85\right)$.

Statistical $\left(f_{C}=.051\right)$

$$
T=1.65 \times 10^{-2}+.34 \times 10^{-2}+.17 \times 10^{-2}=2.16 \times 10^{-2}
$$


Deterministic $\left(\mathrm{f}_{\mathrm{C}}=.048\right)$

$$
T=1.65 \times 10^{-2}+.34 \times 10^{-2}+.06 \times 10^{-2}=2.05 \times 10^{-2}
$$

The optimum $\lambda$ 's found in previous sections are a function of slab thickness, thus requiring normalization of the correction algorithms. This is done by replacing the augmented feature vector $\mathrm{Y}^{*}$ by $\mathrm{Y}^{\prime}$ as given by

$$
\text { unnormalized } Y^{*}=\left[\begin{array}{l}
\mathrm{x} \\
1
\end{array}\right] \quad \text { normalized } Y^{\prime}=\left[\begin{array}{c}
\frac{\mathrm{x}}{\mathrm{L}} \\
\frac{1}{\mathrm{~L}}
\end{array}\right]
$$

This results in

$$
\begin{aligned}
w_{1}=w_{1} \pm c^{\prime} x^{\prime} \text { and } \\
w_{2}=w_{2} \pm \frac{c^{\prime}}{L}, \\
\text { where } c^{\prime}=\frac{\lambda / g^{\prime} \mid}{x^{\prime 2}+\frac{1}{L^{2}}}=L c \\
g^{\prime}=w_{1} x^{\prime}+w_{2} / L \\
x^{\prime}=x / L \\
L=s l a b \text { thickness }
\end{aligned}
$$


for the correction algorithms. Similarly, for the statistical classifier the following should be used:

$$
W_{i+1}=W_{i}-\lambda \cdot \nabla \widetilde{R}^{\prime}\left(W_{i}\right)
$$

where $\lambda^{\prime}=\lambda / L$ and

$$
\left[\begin{array}{c}
\frac{\partial R}{\partial w_{1}} \\
\frac{\partial R}{\partial w_{2}}
\end{array}\right]^{\prime}=\left[\begin{array}{l}
\frac{-w_{2} L}{w_{1}^{2}} \\
\frac{-1}{w_{1} L}
\end{array}\right]
$$

In summary, for the one-dimensional homogeneous slab the deterministic approach appears to have an advantage over the statistical approach because of its reduced variability at early stages. Furthermore, if the variability is used as a threshold for using a decision surface shortly after the approach to such a surface, the deterministic classifier also presents a savings in computer time. However, the variability of the deterministic classifier is bounded by a minimum (depending on the amount of overlapping of the class distributions); whereas the statistical classifier has a variability which is guaranteed to approach zero. 


\section{3) Mu1ti-Region Slab and Initial Conditions}

In the previous section a one region slab was used to demonstrate the type of behavior which might be expected when pattern recognition is used to identify splitting surfaces. Although convenient for demonstratio:. purposes, such problems are little challenge to the skilled Monte Carlo user as far as identifying splitting planes is concerned. If one considers the problem of a muiti-region slab consisting of several layers of different materials, the problem becomes much more difficult for the "human intuition" approach. This section considers this slightly more complex problem using the identical pattern recognition techniques described in the previous section.

The problem is illustrated in Figure 4.17 for a slab consisting of four materials. The FORTRAN coding for the Monte Carlo program used to solve this problem is shown in Appendix G. Figure 4.18 shows the importance distributions for several different combinations of materials (see Table 4.6). Each region is $50 \mathrm{~cm}$ thick and has a total macroscopic cross section of $\Sigma_{t}=.5 / \mathrm{cm}$. The variety of distributions results from rearranging the sequence of the materials and in one case even leads to a bi-modal distribution. The class distributions for these same problems is shown in Figure 4.19 where the median importance has been used for $\overline{\mathrm{I}}$.

Because $\Sigma_{t}$ is the same for all four cases, the collision points of each Monte Carlo run are identical (since each Monte Carlo run uses the same set of random numbers). Therefore the set of feature vectors 


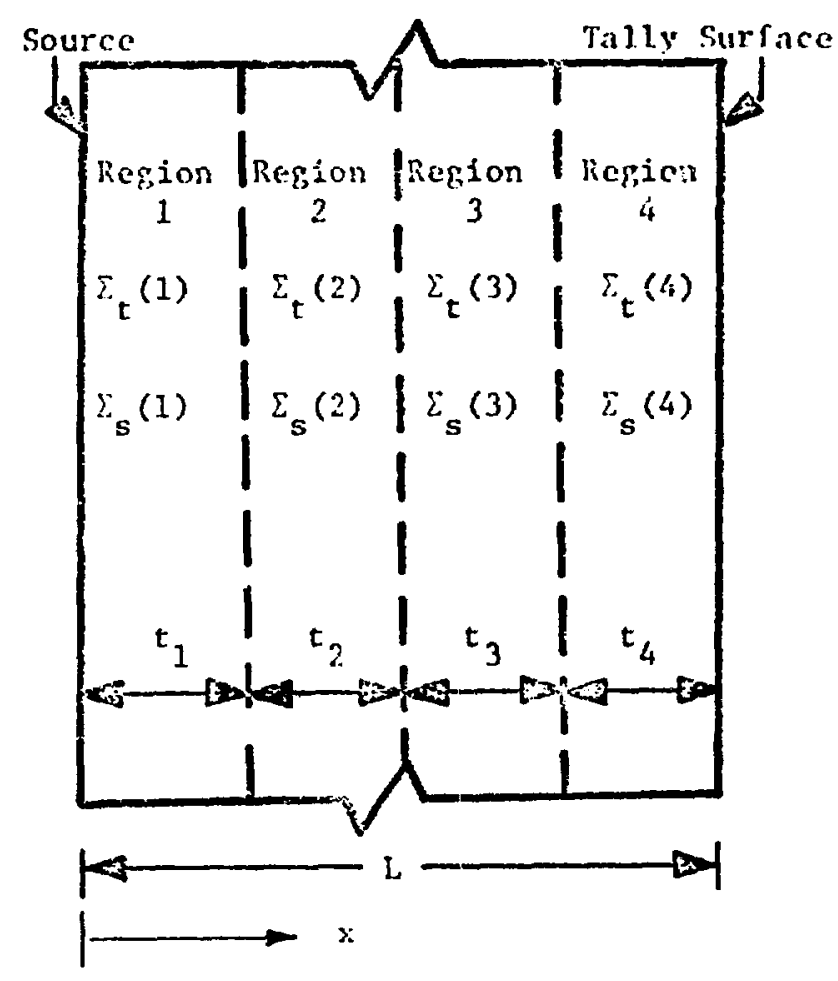

Figure 4.17 The Multi-Region Slab Problem

$Y_{i}$, used for prototypes is identical for each case. The variation in results of the four cases can therefore be attributed solely to the effect of different class and importance distributions as caused by the different $\Sigma_{s}$ 's.

Because particles are allowed to continue through the slab, there is an equal probability throughout the slab of having a collision (and thus a prototype created) between $x$ and $x+d x$. As a result for half the protetypes

$$
y_{1}=x<100
$$




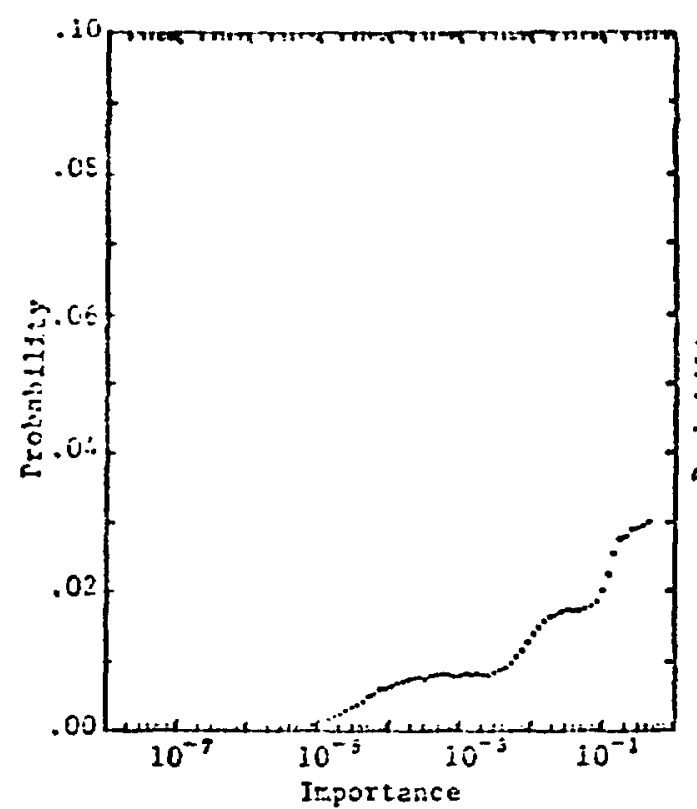

(a) Case I

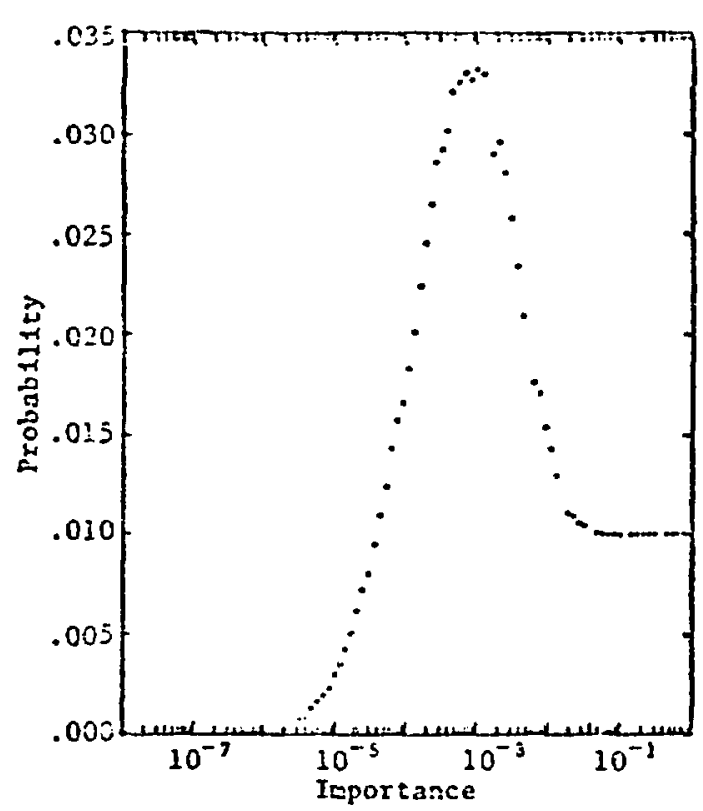

(c) Case III

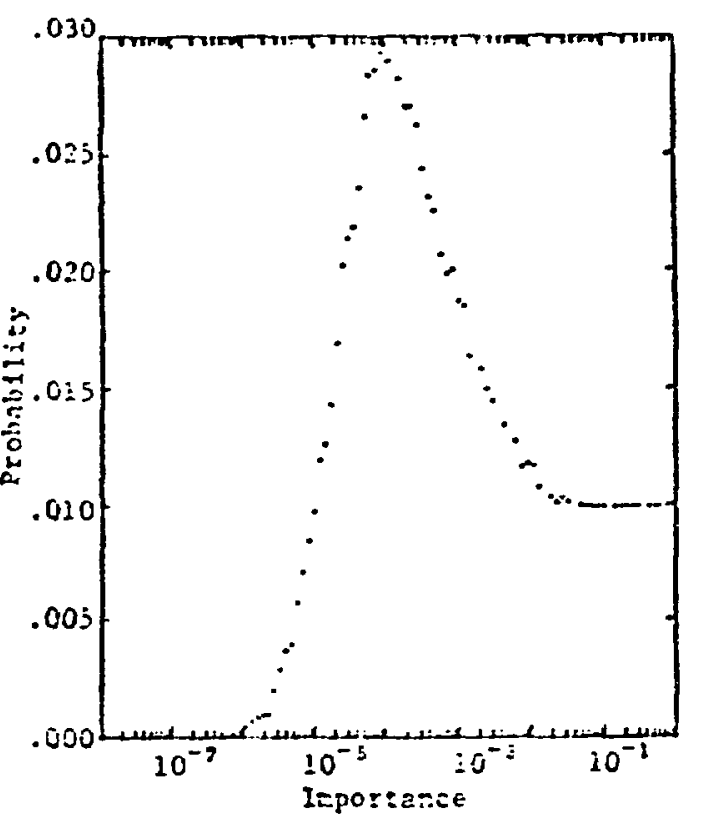

(b) Case II

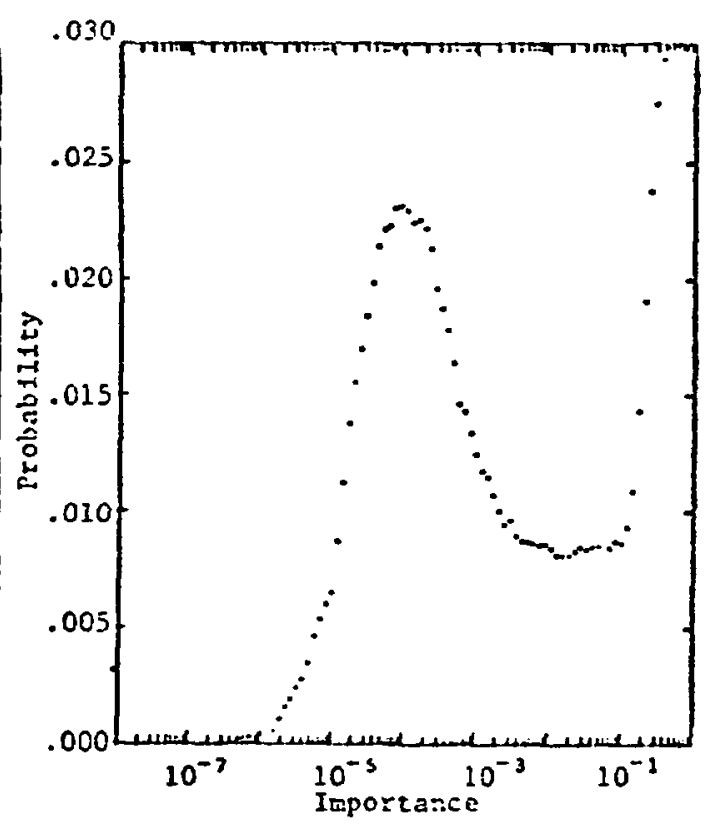

(c) Case IV

Figure 4.18 Importance Distributions for the Multi-Region Slab Problem 


\begin{tabular}{lcccc} 
& CascI & Case II & Case III & Case IV \\
\cline { 2 - 2 }$\Sigma_{s}(1)$ & .40 & .49 & .45 & .49 \\
$\Sigma_{s}(2)$ & .45 & .47 & .49 & .45 \\
$\Sigma_{s}(3)$ & .47 & .45 & .47 & .40 \\
$\Sigma_{s}(4)$ & .49 & .40 & .40 & .47 \\
Ta11y & $6.7 \times 10^{-5}$ & $7.6 \times 10^{-5}$ & $7.5 \times 10^{-5}$ & $7.0 \times 10^{-5}$ \\
Median I & .112 & .00035 & .00105 & .0011 \\
Mean I & .288 & .0502 & .0508 & .140 \\
Er* & 3.2 & 12.6 & 19.1 & 5.6
\end{tabular}

*Using Median $I$ for $\bar{I}$

Table 4.6 Multi-Region Sample Problems

and for the other half

$$
y_{1}=x>100
$$

Therefore the optimum position to locate the splitting surface (using the median $\bar{I}$ ) is the same for $a 11$ four cases and is located at $x=100$. 

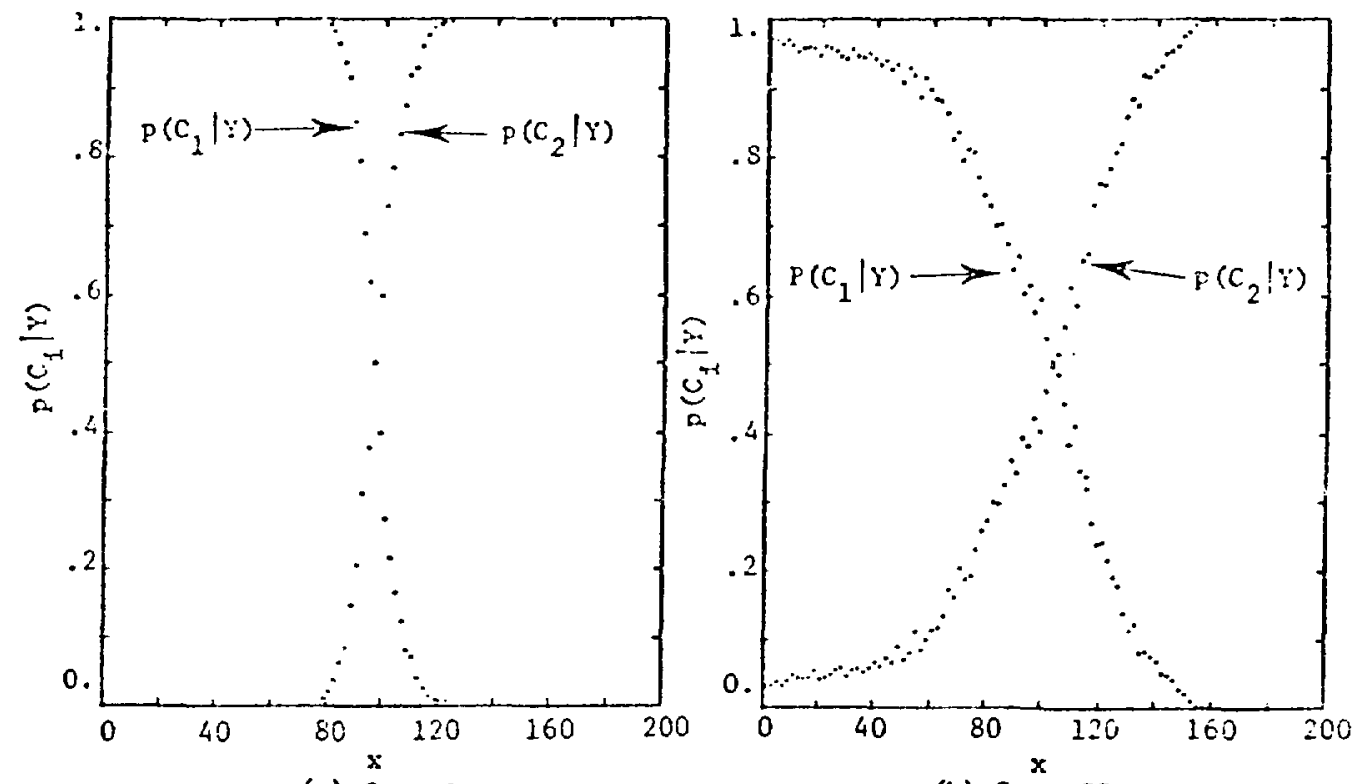

(a) Case I

(b) Case II

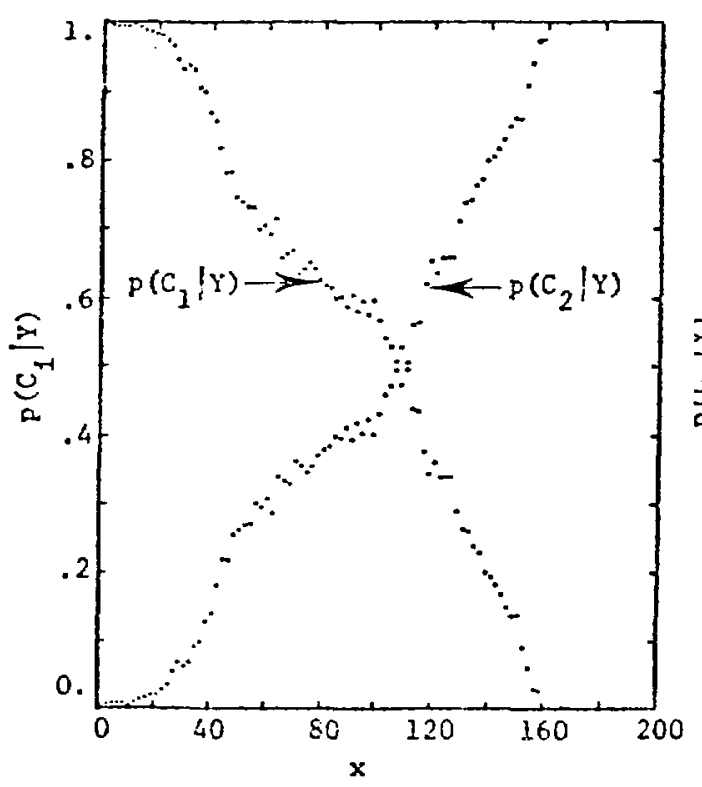

(c) Case III

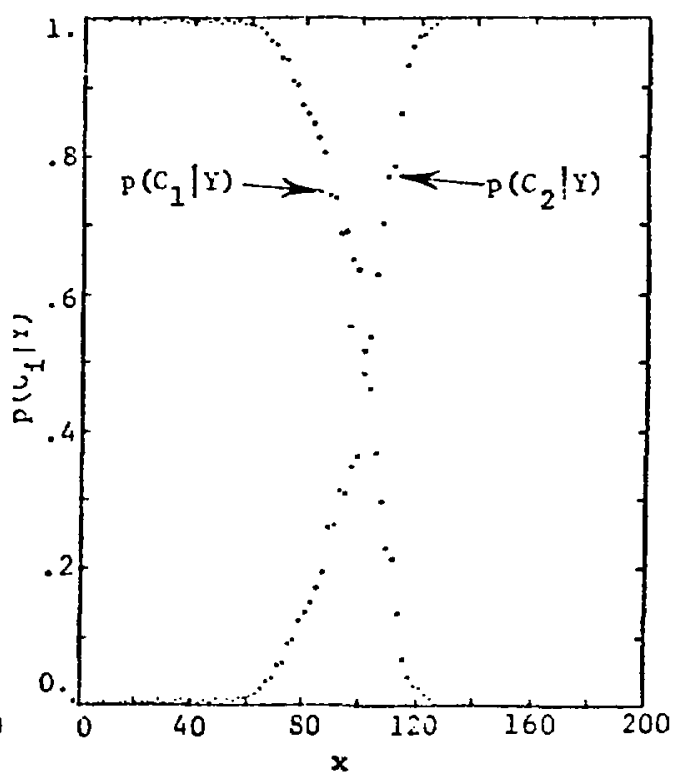

(d) Case IV

Figure 4.19 Class Distributions for the Multi-Region Slab Problem 
An additional set of computational experiments is performed in this section to determine the sensitivity of the pattern classifiers to the selection of an initial decision surface, $s_{1}$, where $s_{1}=-w_{2} / w_{1}$ for the initial selection of $w_{1}$ and $w_{2}$.

\subsection{1) Initial Conditions}

In Section 4.2, the midpoint of the slab was used as the initial iocation of the decision surface. In this section, the effect of the initial location, $s_{1}$, on the performance of several pattern classifiers is analyzed using Case I. Several computation experiments are made using different classification algorithms and different learning parameters, $\lambda$. The initial location can be varied in two ways: (1) varia.tion of the initial value of $w_{1}$ and (2) variation of the initial decision surface location (i.e., $\left.-w_{2} / w_{1}\right)$.

4.3.1a) Deterministic Classifier. The location of the decision surface after the $j$ 'th prototype belonging to $C_{k}$ has been misclassified is given by 


$$
\begin{aligned}
s_{j+1} & =\left(\frac{-w_{2}}{w_{1}}\right)_{j+1}=\left(\frac{-w_{2}+\frac{(-1)^{k+1} \lambda \mid g(Y)}{\left(x^{2}+1\right)}}{w_{1}+\frac{(-1)^{k} \lambda|g(Y)| x}{\left(x^{2}+1\right)}}\right) \\
& =\frac{s_{j}\left(x^{2}+1\right)+\lambda\left(x-s_{j}\right)}{\left(x^{2}+1\right)-\lambda x\left(x-S_{j}\right)}
\end{aligned}
$$

where $x$ is the feature, $y_{1}$, of the $j^{\prime}$ th misclassified prototype. Since $s_{j+1}$ does not depend on $w_{1}$ (independently of $s_{j}$ ), the value of $w_{1}$ for any given $S_{j}$ has no effect upon the behavior of the classifier. Therefore the initial selection of $w_{I}$ (for any given $S_{1}$ ) causes no change in classifier performance. The effect of varying the initial decision surface, $S_{1}$, after 1000 source particles is demonstrated in Figure 4.20 for $10^{-4} \leqslant \lambda \leqslant 1$ and six different $s_{1}$. This figure illustrates that after 1000 source particles the perormance is relatively independent of initial conditions and $\lambda$ for $\lambda>1,{ }^{2}$.

The performance as a function of the number of source particles is show in Figure 4.21 for runs using $\lambda=.05$. In these runs it was found that when $S_{1}$ is selected below the final decision surface location, (i.e. $s_{1}=1,10,50$ ), the decision surface converges monotonically to the final location. This behavior is reflected in Figure 4.21 a by the monotonically decreasing misclassification rate when $s_{1}=1,10$, and 50. However, when $s_{1}$ is started above the final decision surface 


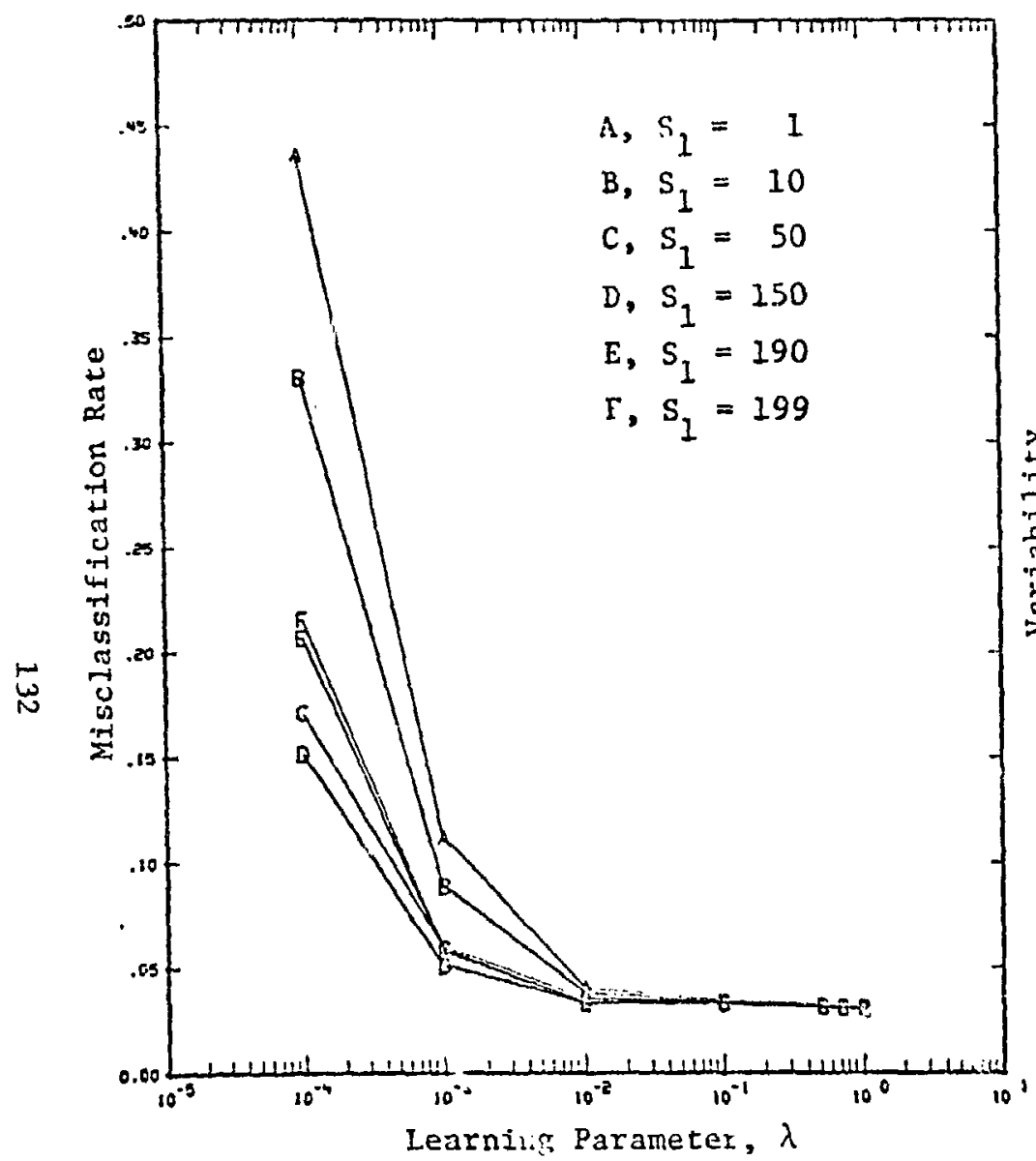

(a) Misclassification Rate

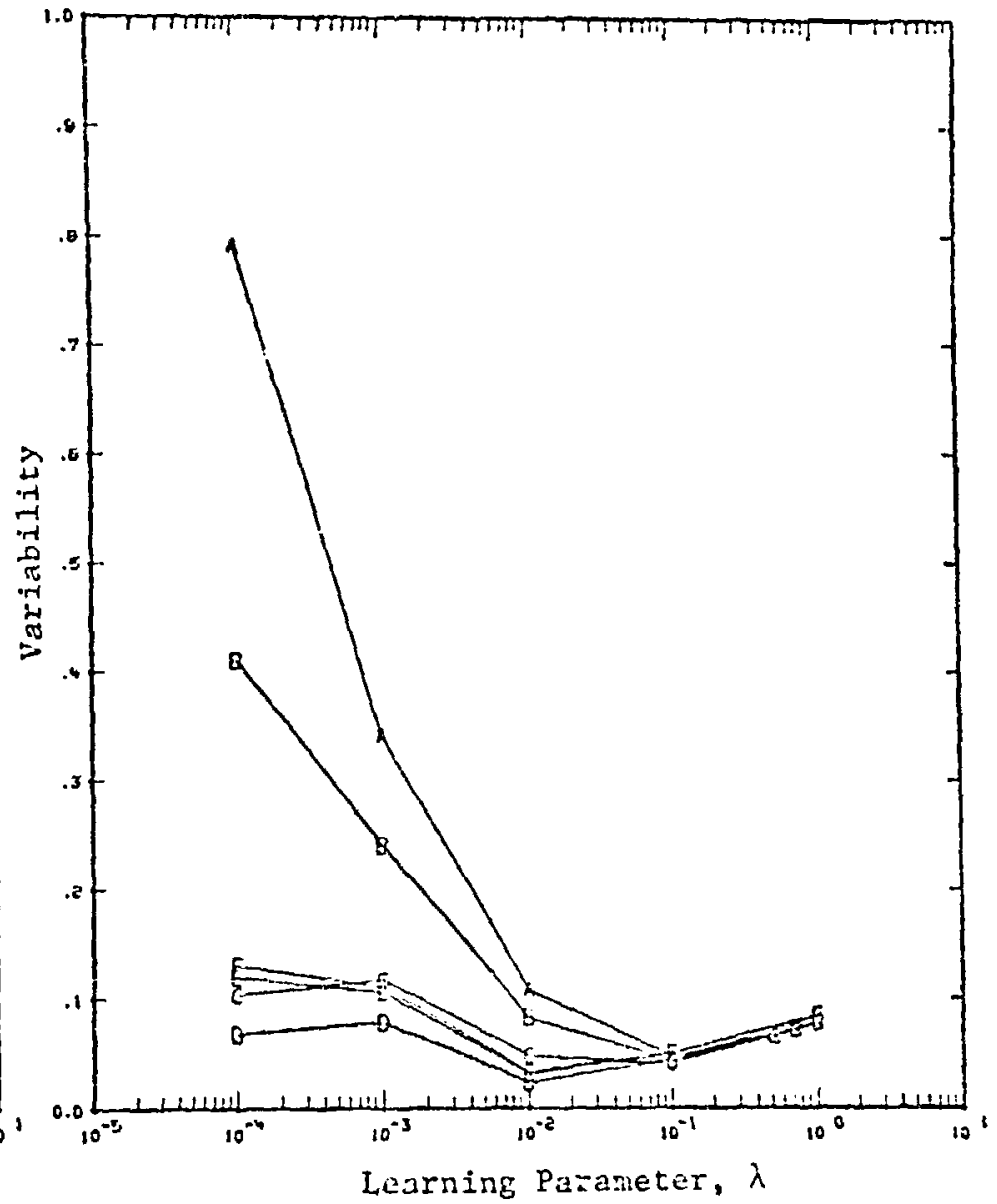

(D) Variability

Figure 4.20 Performance Vs. $\lambda$ for Various Initial Conditions, Deterministic Classifier 


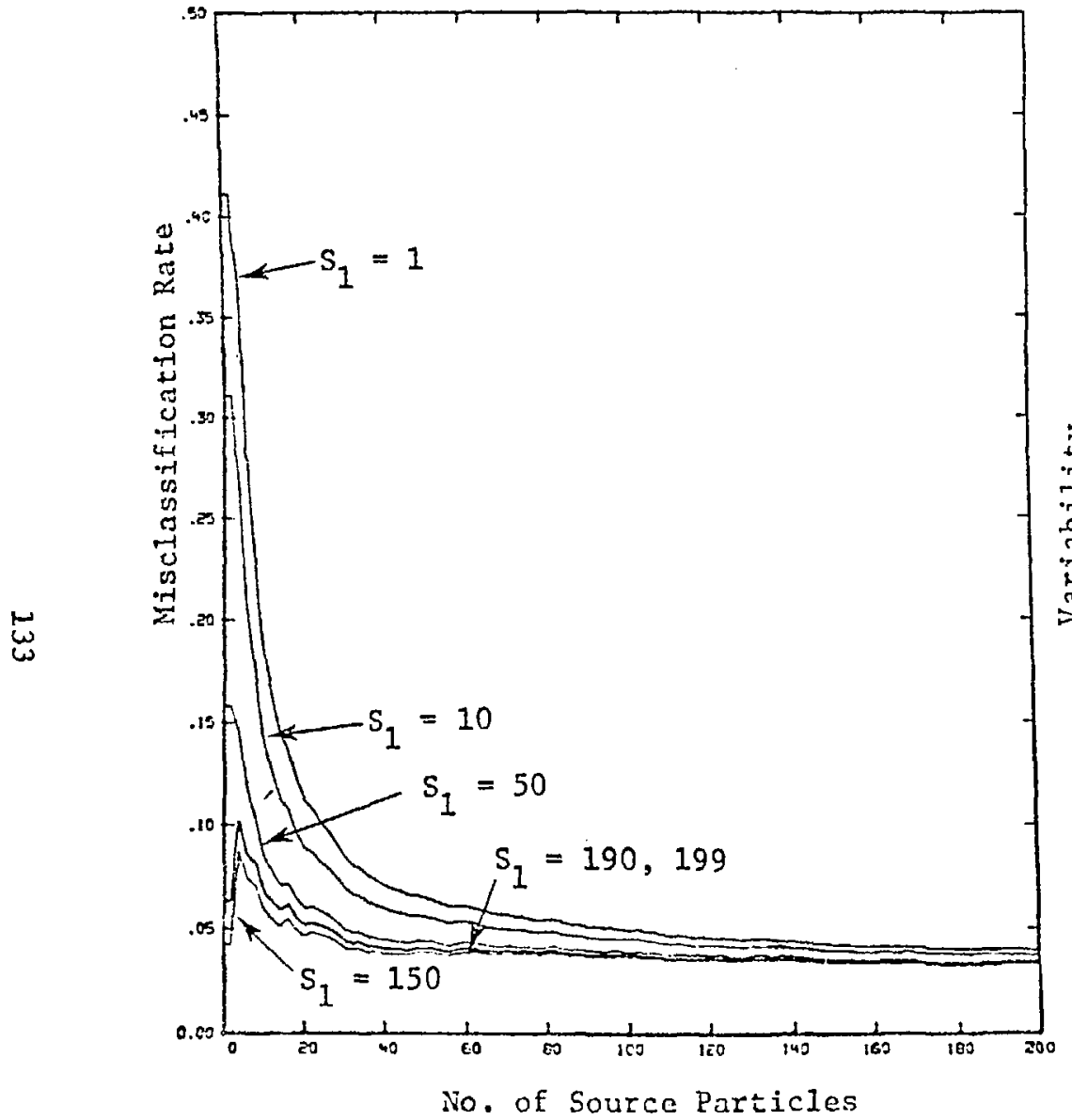

(a) Misclassiffcation Rate

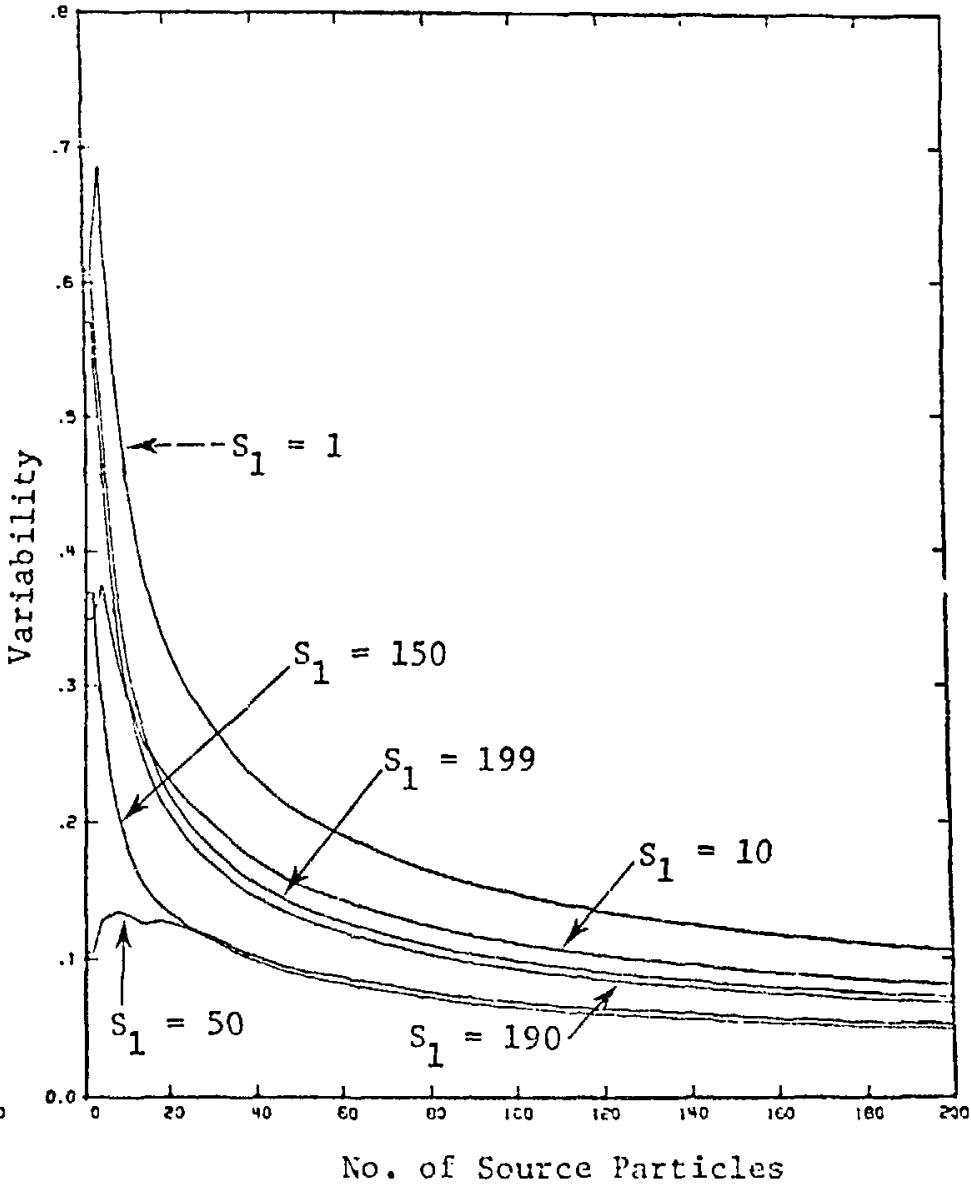

(b) Variability

Figure 4.21 Performance Vs. Number of Source Particles for Various Initial Conditions, Deterministic Classifier 
location (i.e., $S_{1}=150,190,199$ ), it was found that the decision surface very quickly (in less than 10 source particles) moved past the optimum location and then converged to its final location from below. This behavior causes the misclassification rate to increase when the decision surface overshoots the optimum location; however, as the surface begins its final convergence, the misclassification rate decreases. This behavior is demonstrated by the $S_{1}=150,190$, and 199 curves of Figure 4.21. Thus although two $S_{1}$ may be located equidistant from the optimum surface location (for example: $S_{1}=10$ and $s_{1}=190$ ) the $s_{1} 10-$ cated above the surface leads to better classifier performance. This behavior is explained by investigating Equation 4.13 and noting that the change in $S_{j}, \Delta S_{j}$, is affected by the value of the feature $x$ as well as the misclassification distance, $\Delta=\left|x-s_{j}\right|$. Contours of $\Delta s_{j}=\left|s_{j+1}-s_{j}\right|$ using Equation 4.13 are shown in Figure $4.22 *$ for $\Delta=10$, 20 and $\lambda=.01, .05$. This figure iilustrates that for misclassifications in class $C_{1}, \Delta S_{j} \rightarrow 0$ as $S_{j} \rightarrow 0$ which explains the slow convergence when $s_{1} \ll 100$. The increasing slope of $\Delta S_{j}$ as $s_{j} \rightarrow 100$ from the right as opposed to the decreasing slope as $S_{j} \rightarrow 100$ from the left explains why the decision surface overshoots and then approaches from below when $S_{1}=150,190$, and 199 .

* Because of overlapping classes the curves shown in Figure 4.22 actually extend across $S_{j}=100$. However, the class 1 misclassification curve has been plotted only for $s_{j}<100$ and class 2 curve only for $s_{j}>100$. 


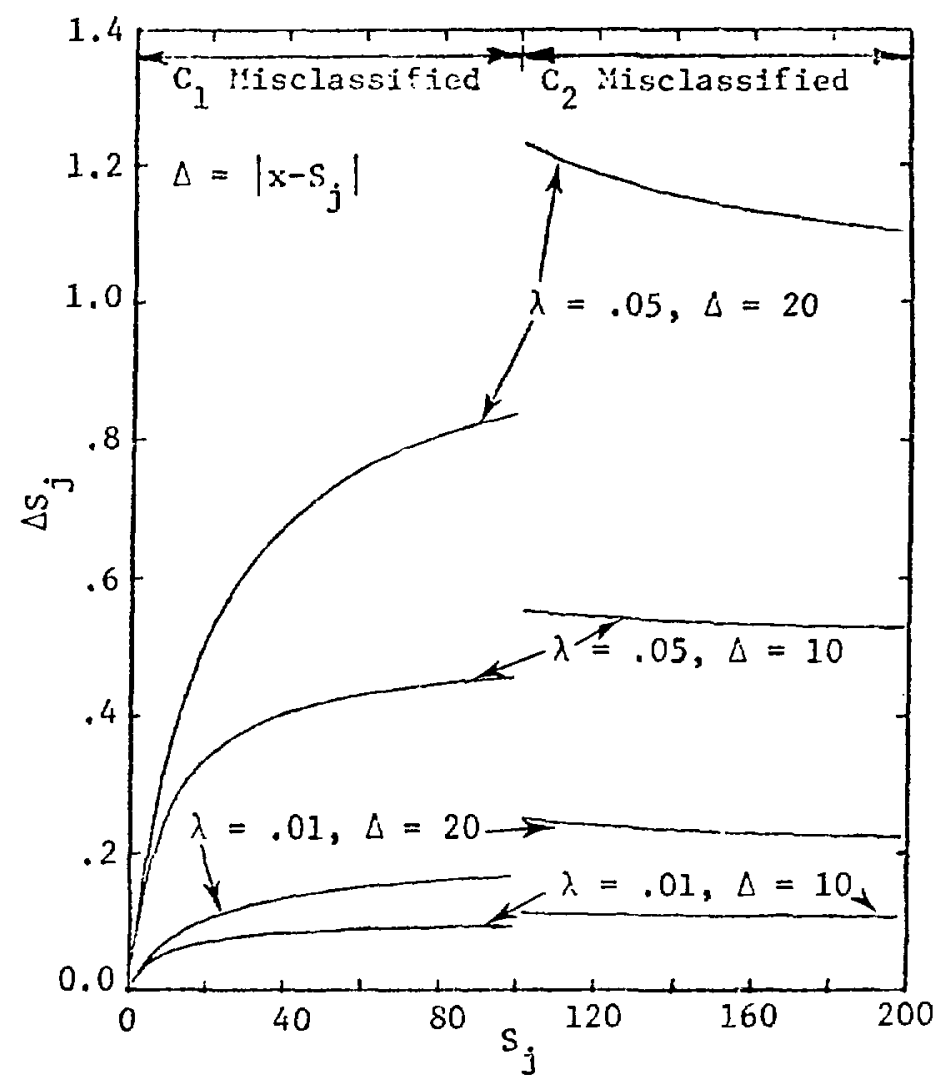

Figure 4.22 Behavior of $\mathrm{S}_{j+1}$ for the Deterministic Classifier

These results indicate that for the deterministic classifier there is a very distinct, advantage to locating $S_{1}$ above the optimum location. 
4.3.1b) Statistical Classicier with loss=d. The decision surzace location after the $j$ 'th prototype belonging to $C_{k}$ has been misclassificd is derived in Appendix $\mathrm{K}$ and is given by

$$
s_{j+1}=\left\{\frac{\left.s_{j} w_{1}+\lambda_{1}-\frac{A_{k, 2}}{\left(M_{k}+1\right)}+\frac{(-1)^{k+1}}{w_{1}\left(M_{k}+1\right)}\right]}{w_{1}-\lambda\left[I_{1}-\frac{A_{k, 1}}{\left(M_{k}+1\right)}+\frac{(-1)^{k+1} S_{j}}{w_{1}\left(M_{k}+1\right)}\right]}\right\}_{j}
$$

where $M_{k}=$ number of prototypes in the $k^{\prime}$ th class (not counting the last misclassified prototype)

$$
\begin{aligned}
A_{k, 1} & =(-1)^{k} \sum_{n=1}^{N_{k}}\left(\frac{w_{2}}{w_{1}}\right)_{n} \\
A_{k, 2}= & (-1)^{k+1} \sum_{n=1}^{N_{k}}\left(\frac{1}{w_{1}}\right)_{n} \\
T_{1}=A_{1,1}+A_{2,1} & A_{2}=A_{1,2}+A_{2,2} \\
N_{k}= & \text { number of misclassified prototypes in the } k^{\prime} t h \\
& \text { class (not counting the last misclassified } \\
& \text { prototype) }
\end{aligned}
$$


Unlike the deterministic classifier, $s_{j+1}$ depends on the selection of an initial $w_{1}$. However, by proper seiection of $\lambda(i . e .$, by keeping $\lambda / w_{1}^{2}=$ constant), this effect can be cancelled. The effect of varying $\lambda / w_{1}^{2}$ is shown in Figure $4.23 a$ for the case where $T_{1}=T_{2}=A_{i, j}=M_{k}=0$. Although, in general $\mathrm{M}_{k} \gg 0$, this figure does illustrate the relative effects of $s_{j}$ and $\lambda / w_{1}^{2}$ on the convergence of the classitier.

Once the decision surface has converged to a final location, $T_{1}$ and $T_{2}$ approach zero. However, due to the overlapping of classes, $\mathrm{A}_{\mathrm{k}, \mathrm{i}}$ approaches a value proportional to the amount of overlapping. Fig$4.23 \mathrm{~b}$ illustrates that this overlapping has very little influence on $\Delta \mathrm{S}_{\mathrm{j}}$. This is to be expected since this term of Equation 4.14 varies as $1 / \mathrm{M}_{\mathrm{k}}^{2}$, whereas the other terms vary as $1 / \mathrm{M}_{k}\left(A_{k, i}\right.$ itself varies as $\left.1 / \mathrm{M}_{k}\right)$. When the decision surface is still approaching a final location, $\mathrm{T}_{1}$ and $\mathrm{T}_{2}$ will not be zero. If the surface is approaching the optimum location from below (i.e., $S_{1}<100$ ), $\mathrm{T}_{1}$ and $\mathrm{T}_{2}$ are positive. The behavior of $\Delta S_{j}$ for this case is illustrated in Figure $4.23 \mathrm{c}$ for $\mathrm{T}_{1}=0,1,10, \mathrm{~T}_{2}=.01 \mathrm{~T}_{1}$, and $\mathrm{M}_{\mathrm{k}}=100,1000$. Similarly the case for $S_{1} \times 100$ is illustrated in Figure 4.23d. These figures illustrate the relative effects of two factors: (1) the statistical effects of past prototypes reflected in $\mathrm{T}_{1}$ and $\mathrm{T}_{2}$ and (2) the effect of the last misclassifjed prototype (the $1 / w_{1}$ and $s_{j} / w_{1}$ terms of Equation 4.14 ). As the misclassification rate of past prototypes becomes large compared with the effects of the last misclassified prototype, 

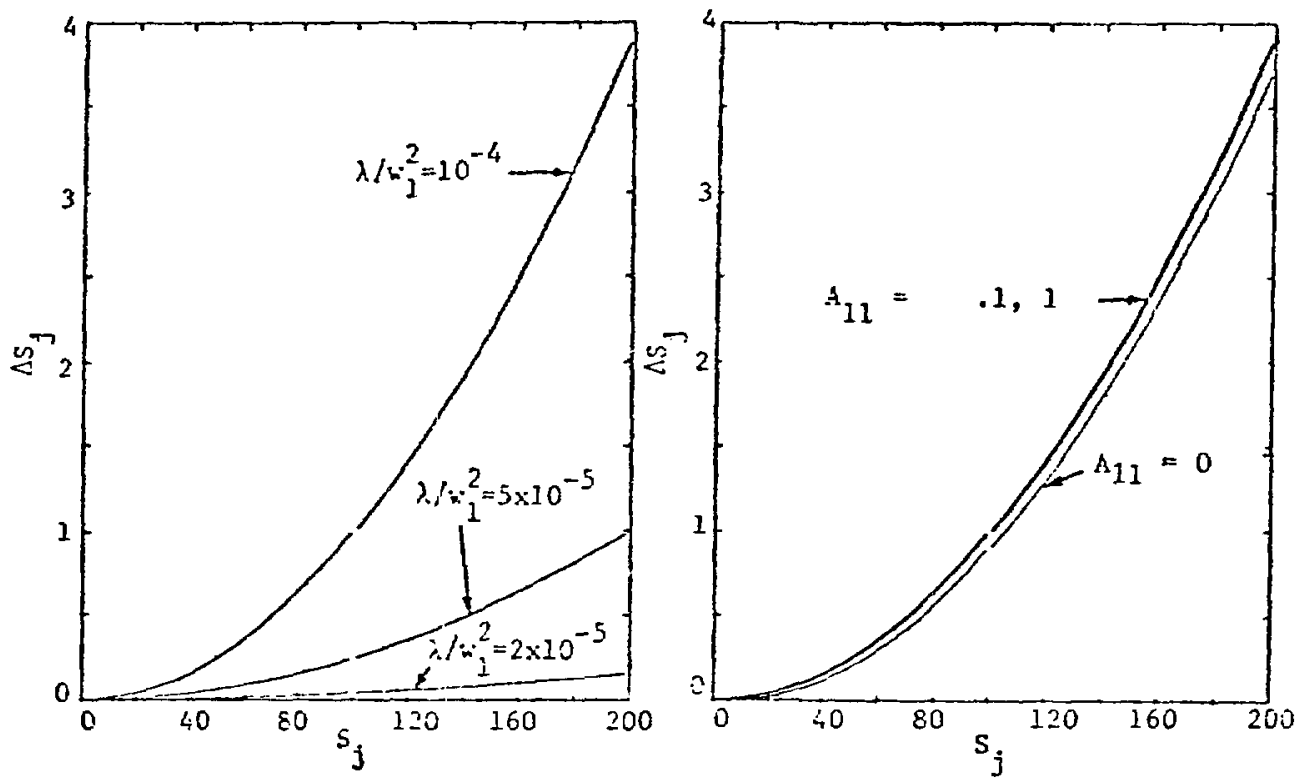

(a) $I_{1}=I_{2}=\dot{A}_{i j}=Y_{k}=0$

(b) $\tau_{1}=T_{2}=H_{k}=0$

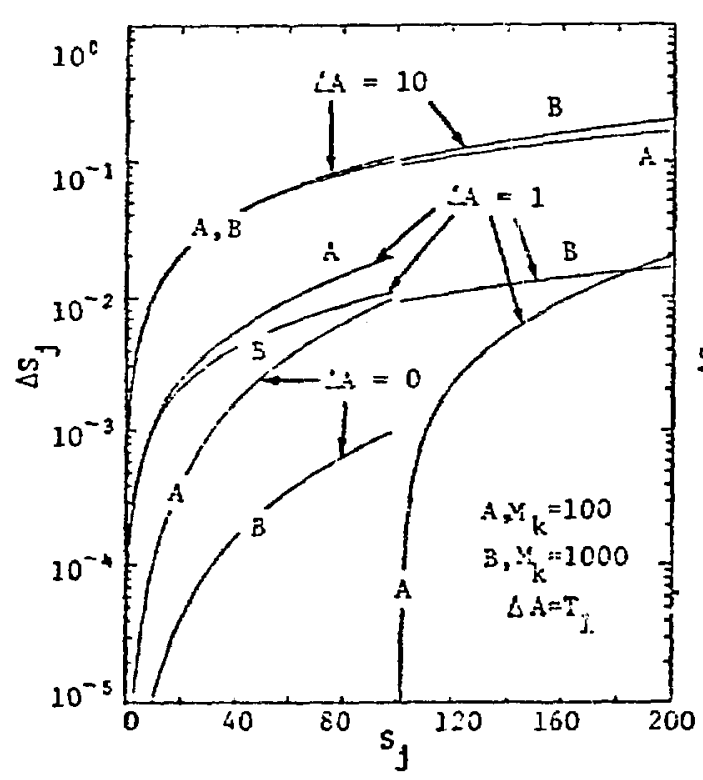

(c) $s_{1}<100, k_{1}=1, \lambda=.0001$

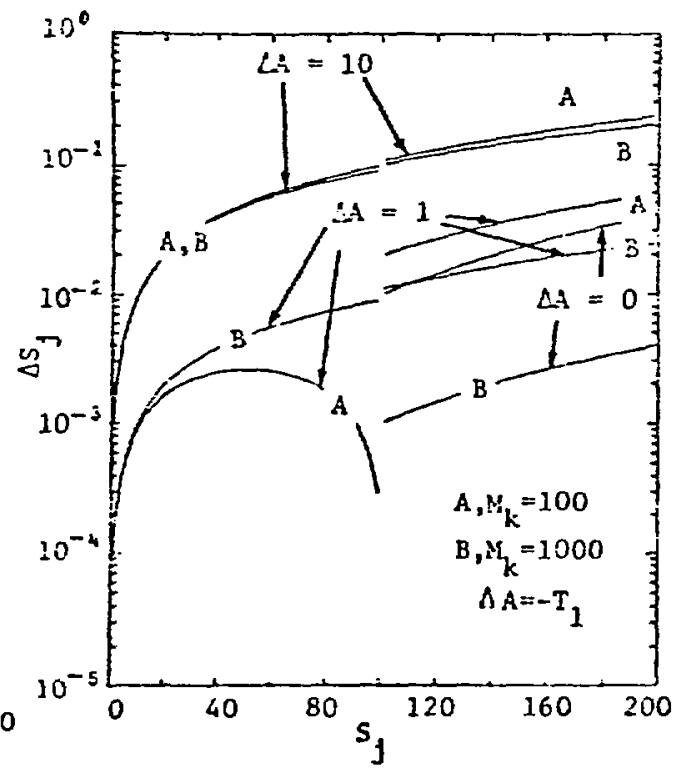

(d) $s_{1}>100, w_{1}=1, \lambda=.0001$

Figure 4.23 Sehavior of $S_{j+1}$ for the Statistical Classifier with Loss $=d$ 


$$
\left|T_{2}\right| \gg\left|\frac{A_{k, 2}}{\left(M_{k}+1\right)}+\frac{(-1)^{k+1}}{w_{1}\left(M_{k}+1\right)}\right|
$$

and

$$
\left|T_{1}\right| \gg\left|\frac{A_{k, 1}}{\left(M_{k}+1\right)}+\frac{(-1)^{k+1} S_{j}}{w_{1}\left(M_{k}+1\right)}\right|
$$

which using Equation 4.14 results in

$$
\Delta s_{j}=s_{j+1}-s_{j} \approx \frac{\lambda\left(T_{2}+T_{1} s_{j}\right)}{w_{1}-\lambda T_{1}}
$$

If it is further assumed that $w_{1} \gg \lambda T_{1}$, and $T_{2} \ll T_{1} S_{j}$ Equation 4.15 reduces to

$$
\Delta \mathrm{S}_{\mathbf{j}} \approx \frac{\lambda \mathrm{T}_{1} \mathrm{~S}_{\mathrm{j}}}{\mathrm{w}_{1}}
$$

Thus $\Delta S_{1}$ varies linearly with $S_{j}$ as is demonstrated in Figures $4.23 \mathrm{c}$ and $4.23 \mathrm{~d}$ by the curves for which $\left|\mathrm{T}_{1}\right|=10$.

When past misclassification becomes small (i.e., after convergence) 


$$
\begin{aligned}
& \left|T_{1}\right| \ll\left|-\frac{A_{k, 1}}{\left(M_{k}+1\right)}+\frac{(-1)^{k+1} S_{j}}{w_{1}\left(M_{k}+1\right)}\right| \\
& \text { and } \\
& \left|T_{2}\right| \ll\left|-\frac{A_{k, 2}}{\left(M_{k}+1\right)}+\frac{(-1)^{k+1}}{w_{1}\left(M_{k}+1\right)}\right|
\end{aligned}
$$

And since in general

$$
\begin{aligned}
& \left|\frac{A_{k, 1}}{\left(M_{k}+1\right)}\right| \ll\left|\frac{s_{j}}{w_{1}\left(M_{k}+1\right)}\right| \\
& \left|\frac{A_{k, 2}}{\left(M_{k}+1\right)}\right| \ll\left|\frac{1}{w_{1}\left(M_{k}+1\right)}\right|
\end{aligned}
$$

Equation 4.14 results in

$$
\Delta s_{j} \approx \frac{(-1)^{k+1} \lambda\left(1+s_{j}^{2}\right)}{w_{1}^{2}\left(M_{k}+1\right)-(-1)^{k} \lambda s_{j}}
$$


If $i t$ is further assumed that $s_{j}^{2} \gg 1$ and $w_{1}^{2}\left(M_{k}+1\right) \gg \lambda s_{j}$, Equation 4.17 reduces to

$$
\Delta s_{j} \approx \frac{\lambda(-1)^{k+1} s_{j}^{2}}{w_{1}^{2}\left(M_{k}+1\right)}
$$

Equation 4.18 indicates that as $T_{i} \rightarrow 0 \quad(i=1,2), \Delta S_{j}$ varies quadratically with $s_{j}$. However, unlike Equation 4.16 , the $\Delta s_{j}$ given by Equation 4.18 goes to zero as $\mathrm{M}_{k}$ increases. This behavior is illustrated by the curves in Figures $4.23 \mathrm{c}$ and $4.23 \mathrm{~d}$ for which $\mathrm{T}_{1}=0$. For these curves, the effect of $\mathrm{M}_{k}$ is much more pronounced than the curves for which $\left|\mathrm{T}_{1}\right|=10$. A1though $\Delta S_{j}$ as given by Equation 4.18 varies quadratically with $S_{j}$ as opposed to 1inearly for Equation 4.16, the coefficient of $\mathrm{s}_{j}{ }^{2}$ is much smaller than the coefficient of $s_{j}$, and the linear term dominates. Thus in Figures $4.23 \mathrm{c}$ and $4.23 \mathrm{~d}$, the $\Delta \mathrm{S}_{j}$ for $\left|\mathrm{T}_{1}\right|=10$ is much greater than for $\left|\mathrm{T}_{1}\right|=0$.

When neither the past misclassification term nor the current misclassified prototype term is dominant, $\Delta S_{j}$ varies proportional to the difference between the two effects. This is illustrated by the $\left|T_{1}\right|=1$ curves in Figures $4.23 \mathrm{c}$ and $4.23 \mathrm{~d}$.

The performance of the classifier after 1000 source particles for six different $S_{1}$ is shown in Figure 4.24 for a rarge of $\lambda$ from $10^{-6}$ 


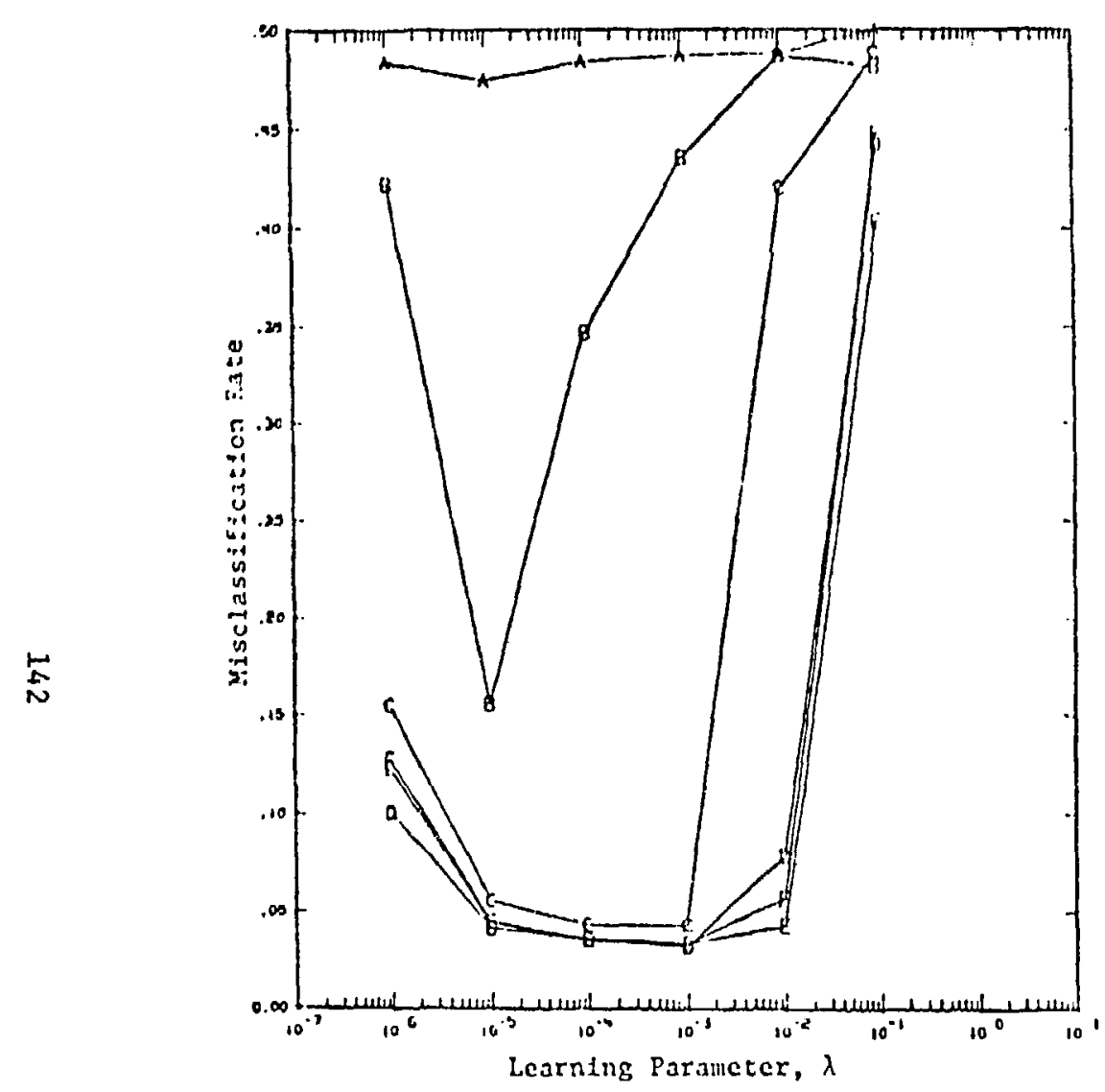

(a) Mlacliticill leation Rate

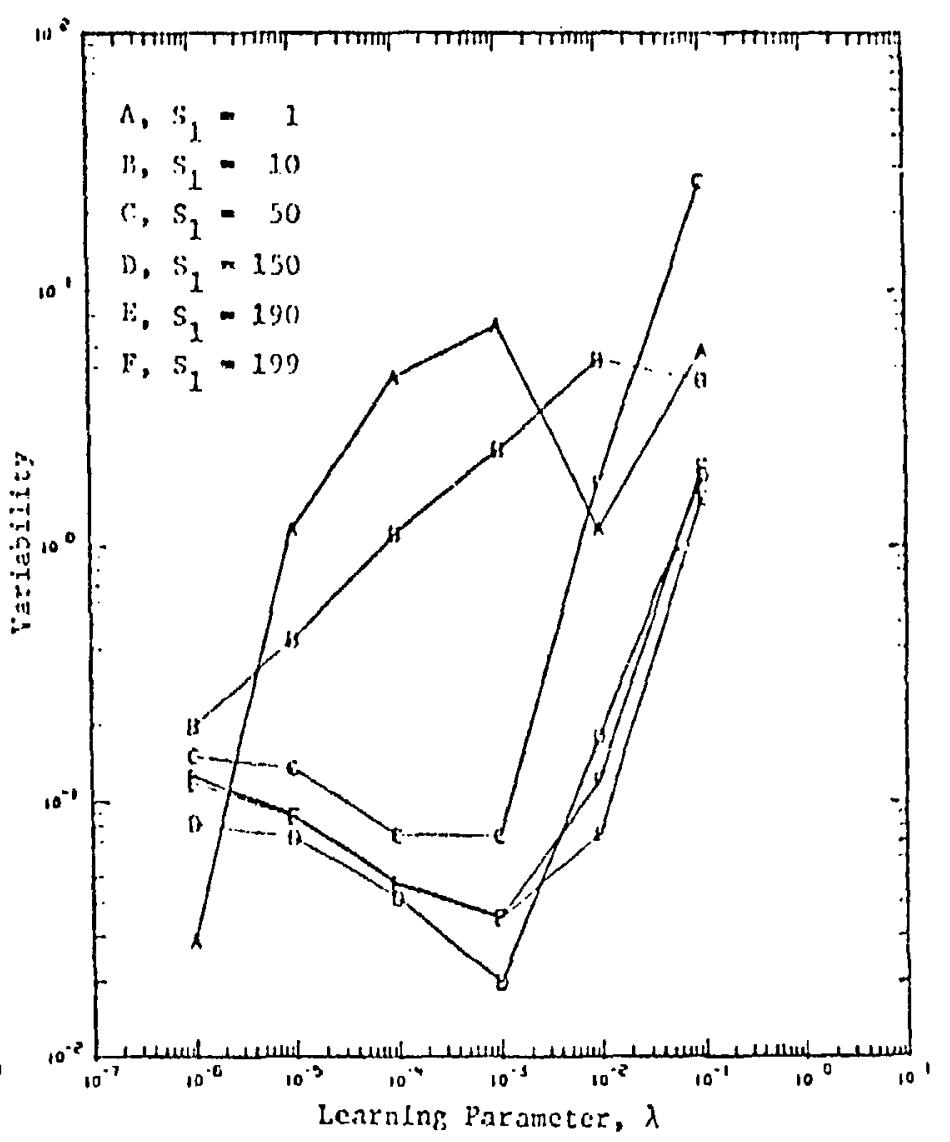

(b) Virliability

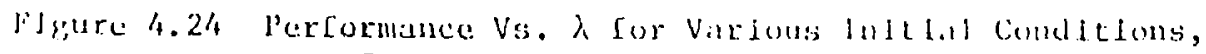
Stotistical Classiffer with Loss $=d$ 
to $10^{-1}$. Unlike the deterministic classifier there is no value of $\lambda$ for which the performance is acceptable for all $\mathrm{S}_{1}$.

The performance as a function of the number of source particles is shown in Figure 4.25 using $\lambda=.0001$ (for $s_{1}=50,150,190,199$ ) and $\lambda=.00001$ (for $s_{1}=1,10$ ). The behavior of the curves for $s_{1}<100$ can be explained as follows. When $S_{1}$ is very small, $\Delta S_{j}$ is also very small as explained in the previous analysis of Equation 4.14. For many iterations there is little change in $S_{j}$ and thus the misclassification rate remains approximately constant (see $S_{1}=1,10$ curves of Figure 4.25a). Although $\Delta S_{j}$ is small, the variability increases since $s_{j}$ is also very small (see $S_{1}=1,10$ curves of Figure 4.25b). Eventually $w_{1}$ is decreased to such an extent that $1 / w_{1}$ and $1 / w_{1}^{2}$ begin to increase rapidly, resulting in large $\Delta S_{j}$ which eventually leads to a large overshoot of the optimum surface location. The magnitude of the overshoot and the following oscillations about the optimum decision surface location depends upon the value of $\mathrm{S}_{1}$. The variability is a good indicator of when this overshoot occurs. Figure $4.25 \mathrm{~b}$ illustrates that for: (a) $S_{1}=50$, the overshoot occurs between 20 and 40 source particles (b) $S_{1}=10$, although $S_{j}$ is increasing rapidly the overshoot does not occur until after 200 source particles (c) $s_{1}=1, s_{j}$ has not even begun its approach to the overshoot.

The primary reason for this difficulty is that Equation 4.14 is not dependent upon the location, $x$ or $\left|s_{j}-x\right|$, of the prototype but is strongly dependent on the current location of the decision surface, $s_{j}$. litis problem can be alleviated by using a $\lambda$ that varies with the 


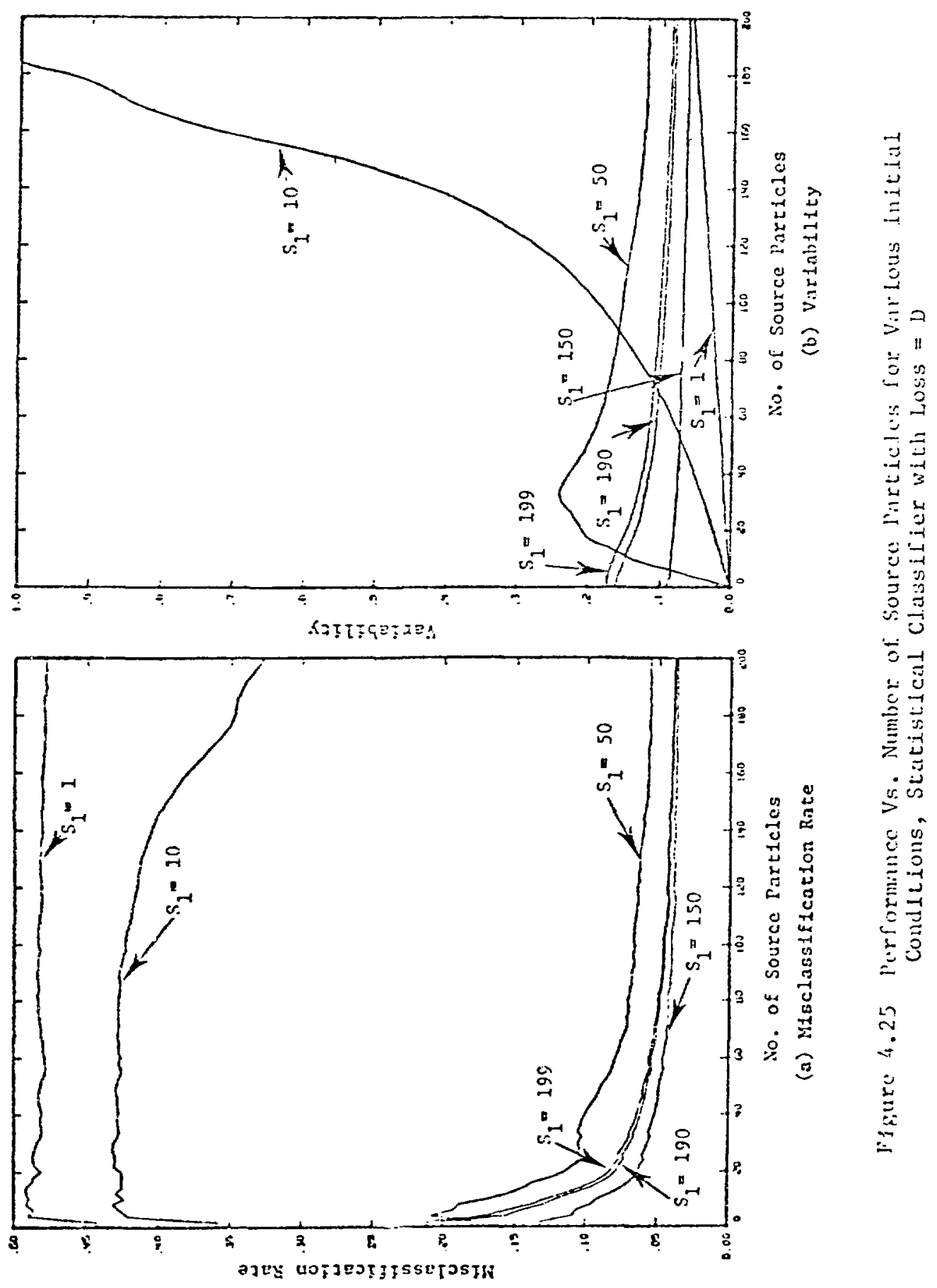


location of $\mathrm{S}_{j}$; however, this would lead to other complexities and would involve the use of more computer time. Because of this difficulty another statistical classifier is investigated which uses a different loss function.

4.3.1c) Statistical Classifier with Loss $=\mathrm{D}$. The derivation of $\mathrm{s}_{j+1}$ with loss $=\mathrm{D}$ (see Table 4.4) is similar to that of $\mathrm{S}_{\mathrm{j}+1}$ using loss $=\mathrm{d}$ (see Appendix $K$ ) and results in

$S_{j+1}=\left\{\frac{S_{j} w_{1}+\lambda\left[T_{2}-\frac{A_{k, 2}}{\left(M_{k}+1\right)}+\frac{(-1)^{k+1}}{\left(M_{k}+1\right) \sqrt{1+x^{2}}}\right]}{w_{1}-\lambda\left[T_{1}-\frac{A_{2} 1}{\left(M_{k}+1\right)}+\frac{x(-1)^{k+1}}{\left(M_{k}+1\right) \sqrt{1+x^{2}}}\right]}\right\}_{j}$,

$$
\text { where } A_{k, 2}=\frac{(-1)^{k+1} \sum_{n=1}^{N_{k}}\left(\frac{1}{\sqrt{1+x^{2}}}\right)_{n}}{(-1)^{k+1} \sum_{n=1}^{M_{k}}\left(\frac{x}{\sqrt{1+x^{2}}}\right)_{n}} \text {, and }
$$

$$
x=y_{1}=\text { distance from the source, }
$$


and all other parameters are identical to those found in Equation 4.14. Unlike the statistical classifier with loss $=d, s_{j+1}$ for this classifier depends on the location of the prototype, $x$, as well as the current position of the decision surface, $s_{j}$. The dependence of $s_{j+1}$ on an initial selection of $w_{1}$ can be eliminated by keeping $\lambda / w_{1}$ constant. When past misclassification effects are large

$$
\begin{aligned}
& \left|T_{2}\right| \gg\left|\frac{-A_{k, 2}}{\left(M_{k}+1\right)}+\frac{(-1)^{k} x}{\left(M_{k}+1\right) \sqrt{1+x^{2}}}\right| \text { and } \\
& \left|T_{1}\right| \gg\left|\frac{-A_{k, 1}}{\left(M_{k}+1\right)}+\frac{(-1)^{k+1}}{\left(M_{k}+1\right) \sqrt{1+x^{2}}}\right| \text {, }
\end{aligned}
$$

and Equation 4.19 can be reduced the same as for the loss $=\mathrm{d}$ classifier resulting in Equation 4.16. Thus for the initial approach to the decision surface, this classifier behaves the same as the loss $=\mathrm{d}$ classifier.

When the decision surface is converging to a final surface

$$
\left|T_{2}\right| \ll\left|\frac{-A_{k, 2}}{\left(M_{k}+1\right)}+\frac{(-1)^{k} x}{\left(M_{k}+1\right) \sqrt{1+x^{2}}}\right| \text { and }
$$




$$
\left|T_{1}\right| \ll\left|\frac{-A_{k, 1}}{\left(M_{k}+1\right)}+\frac{(-1)^{k+1}}{\left(M_{k}+1\right) \sqrt{1+x^{2}}}\right| .
$$

Through the assumptions that

$$
\begin{aligned}
& \left|\frac{-A_{k, 1}}{\left(M_{k}+1\right)}\right| \ll\left|\frac{(-1)^{k+1}}{\left(M_{k}+1\right) \sqrt{1+x^{2}}}\right| \text { and } \\
& \left|\frac{-A_{k, 2}}{\left(M_{k}+1\right)}\right| \ll\left|\frac{(-1)^{k} x}{\left(M_{k}+1\right) \sqrt{1+x^{2}}}\right|,
\end{aligned}
$$

Equation 4.19 can be reduced to

$$
\Delta s_{j} \approx \frac{\lambda(-1)^{k+1}\left(1+s_{j} x\right)}{w_{l}\left(M_{k}+1\right) \sqrt{1+x^{2}}-\lambda(-1)^{k+1} x}
$$

and under the further assumptions that

$$
\mathrm{S}_{\mathrm{j}} \mathrm{x} \gg 1, \sqrt{1+\mathrm{x}^{2}} \approx \mathrm{x} \text {, and } \lambda \ll\left|\mathrm{w}_{1}\left(\mathrm{M}_{k}+1\right)\right|
$$


it can be reduced to

$$
\Delta s_{j} \approx \frac{\lambda(-1)^{k+1} s_{j}}{w_{1}\left(M_{k}+1\right)}
$$

A comparison of Equation 4.21 with Equation 4.18 shows that using loss $=D$ linearizes the dependence of $\Delta S_{j}$ on $S_{j}$ for the case when the decision surface is undergoing the final convergence. However, Equation 4.21 indicates that the dependence of $\Delta S_{j}$ on $s_{j}$ on $x$ (as shown by Equation 4.19) cancels out (based upon the assumptions made to obtain Equation 4.21). A plot of Equation 4.19 for $A_{k, i}=M_{k}=0$ is shown in Figure 4.26 for three values of $\mathrm{w}_{1}$ and $\lambda=.05$. The discontinuity of these curves is caused by the $-\lambda$ term in the denominator of Equation 4.20 and becomes less pronounced as $w_{1}\left(M_{k}+1\right)>\lambda$.

Plots of Equation 4.19 for various values of $\mathrm{A}_{k, i}, \mathrm{M}_{k}$, etc. are not presented here but are very similar to the plots of Figure $4.23 \mathrm{c}$ and 4.23d. This is because, like the loss $=d$ classifier, the coefficient of $s_{j}$ in Equation 4.21 is small compared with the coefficient of $s_{j}$ in Equation 4.16.

The performance of the loss $=D$ classifier is shown in Figure 4.27 for several $\mathrm{s}_{1}$ and a range of $\lambda, 10^{-6} \leqslant \lambda \leqslant 10$. Although the misclassification rate is similar to the loss $=\mathrm{d}$ case, the variability for small $\mathrm{s}_{1}$ has increased considerably. 


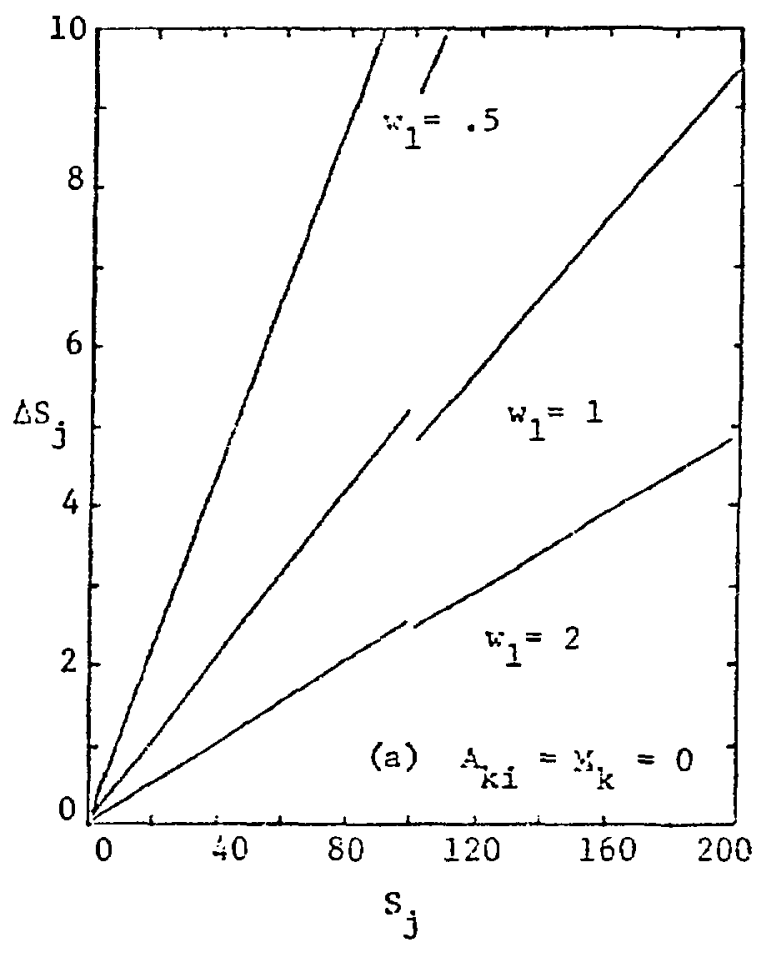

Figure 4.26 Eenavior of $S_{j+1}$ far the Statistical Classifier with Loss $=\mathrm{D}$

Figure 4.28 shows the performance for the first 200 source particles using $\lambda=.05$. The extrene oscillations for the $\mathrm{S}_{1}=1$ curve can be explained as follows. During initial operation, $w_{1}$ is initially decreased. Like the loss $=d$ classifier as $w_{1}$ decreases, $\dot{y}_{1}$, Increases. This positive feedback can lead to an instability as $v_{1} \rightarrow 0$ and $w_{1}$ can actually become negative. In this case $S_{j}$ changes sign which leads to a very large variability as seen by Figure $4.28 \mathrm{~b}$. 


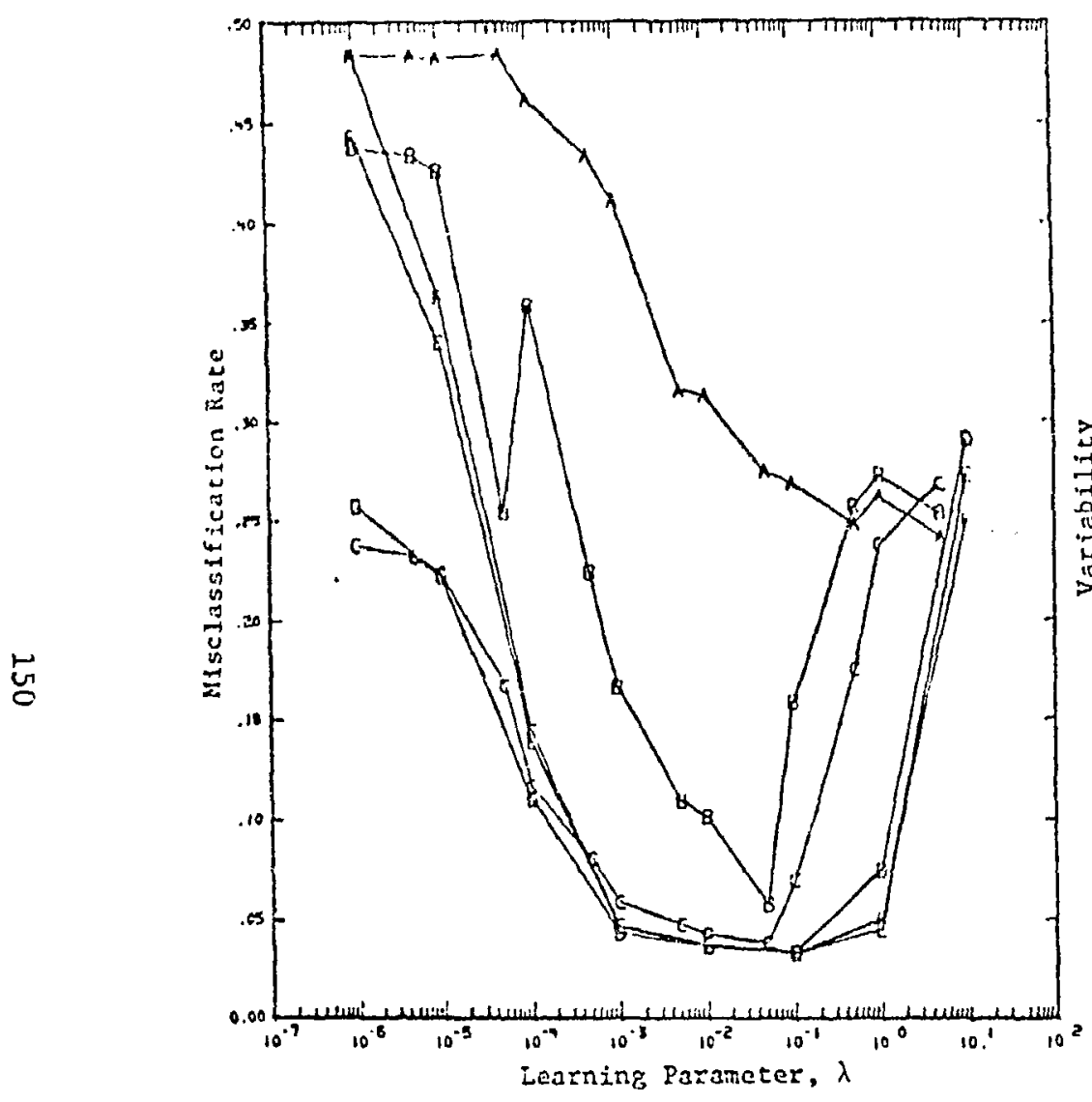

(a) Misclassification Rate

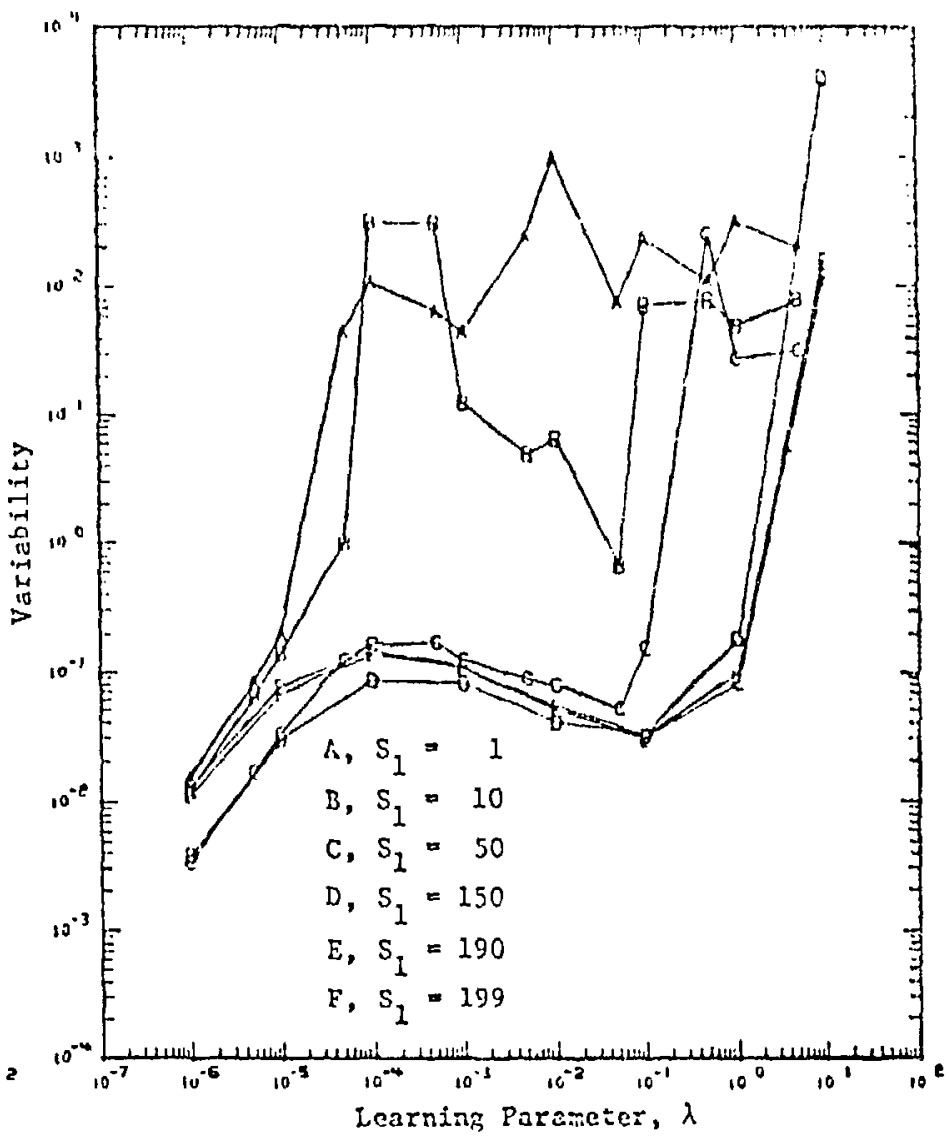

(b) Variabilicy

Figure 4.27 Performance Vs. $\lambda$ for Various Initial Conditions, Statistical Classifier with Loss $=D$ 


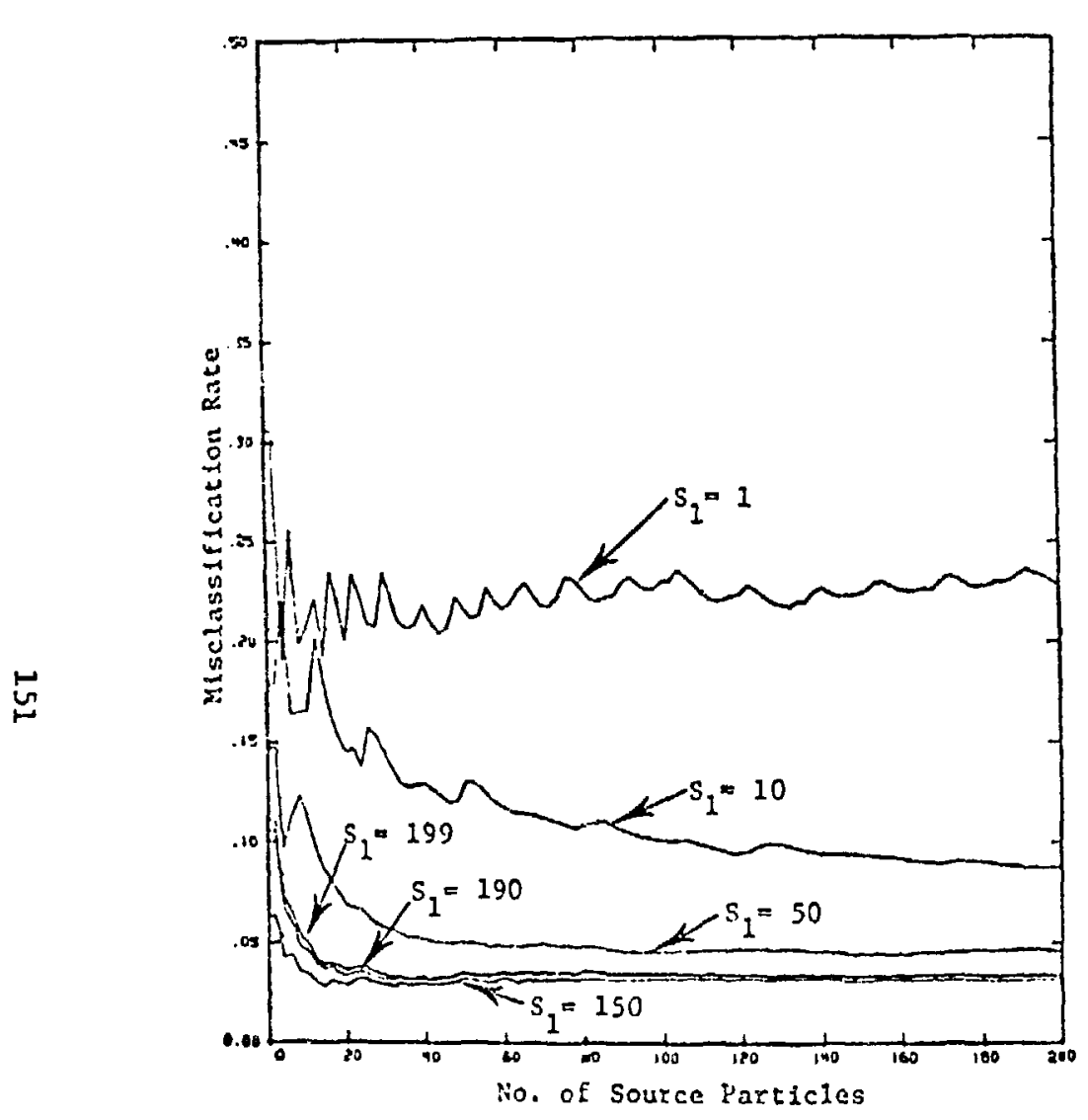

(a) Misclassification Rate

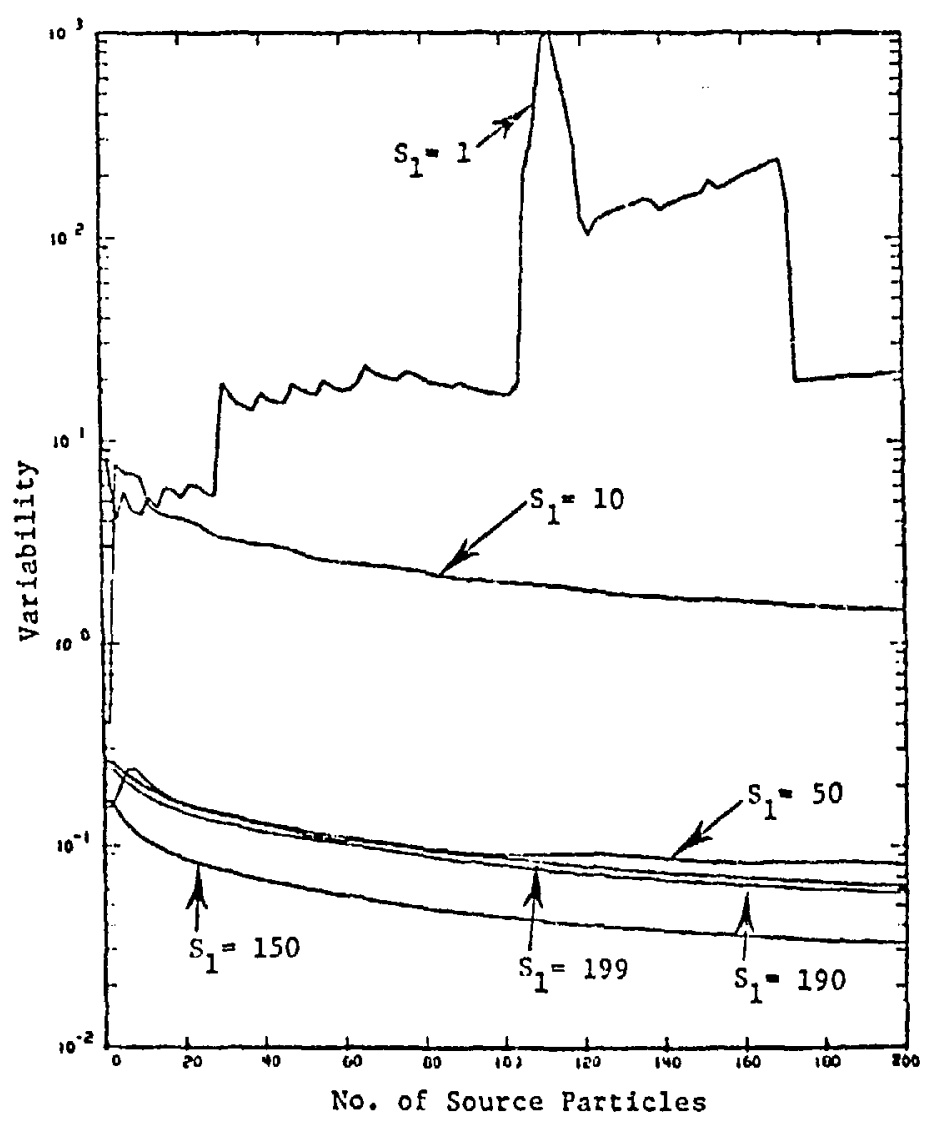

(b) Varlability

Figure 4.28 Performance Vs. Number of Source Particles for Various Initial Conditions, Statistical. Clessifier with Loss $=\mathrm{D}$ 
In summary, although by using loss $=1, \Delta S_{j}$, has been made to depend on $x$, the dependence is very weak. The classifier is therefore no improvement over the loss $=\mathrm{d}$ classifier for small $\mathrm{s}_{1}$.

4.3.1d) Sumary. It has been found in this section that the performance of the various classifiers does depend strongly on the choice of an initial $s_{1}$. The deterministic classifier is far superior to the statistical ones with regard to initial condition selection below the final. decision surface. However, when $S_{1}$ is chosen above the final surface, the deterministic and statistical techniques are competitive.

It has also been found that the statistical techniques do depend on $w_{1}$. However, by using an appropriate (for loss $=d, \lambda / w_{1}^{2}=$ constant; for $\operatorname{loss}=\mathrm{D}, \lambda / \mathrm{w}_{1}=$ constant), the effect of selecting an initial $w_{1}$ can be eliminated.

It should be noted that although the statistical classiffer appears to perform poor $1 y$ for small $s_{1}$, this effect can be eliminated by using the distance from the detector $(L-x)$ instead of the distance from the source $(x)$ in the feature vector prototypes. This would necessitate changing the initial weight to correspond to the new feature space. This will be done for the two-dimensional problem of Section 4.4. This new feature selection would allow the Inftialization of splitting surfaces at the origin $\left(s_{j}=0\right)$; however, it would prohibit originating at $S_{j}=L$. 


\subsection{2) MuIti-Region Slab Variations}

In this section, three different classifiers (deterministic, statistical with loss $=d$, and statistical with loss $=$ D) will be used to identify splitting planes or decision surfaces for the four Monte Carlo problems described in Table 4.6. The median importances of the distributions shown in Figure 4.18 will be used to separate the classes resulting in the class distributions shown in Figure 4.19. In the previous section it was found that a high value for the initial decision surface is better than a low one. Thereforean $s_{1}$ of 200 will be used in a11 cases.

Because of the different types of distributions that can occur (see Figures 4.18 and 4.19 ) the optimal $\lambda$ for different problems varies (see Figures 4.29 and 4.30). If two classes are entirly non-overlapping, a $\lambda$ which is large enough to compensate completely for a misclassified prototope is desirable since no prototype will ever appear to belong to more than one class. However, as classes begin to overlap, the amount of correction and thus $\lambda$ must be decreased because falsely labeled prototypes could lead to a large variability of the decision surface. This phenomonon is apparent from Figures 4.29 and 4.30 which show the results after 100 particles. In the deterministic case the misclassification rate decreases for increasing $\lambda$, but the variability increases. In Cases II and III the overlapping of classes results in a high variability which is understandable since the deterministic classifier is not designed for overlapping classes. The statistical classifier leads to a large improvement in variability for Cases II and III but only a 

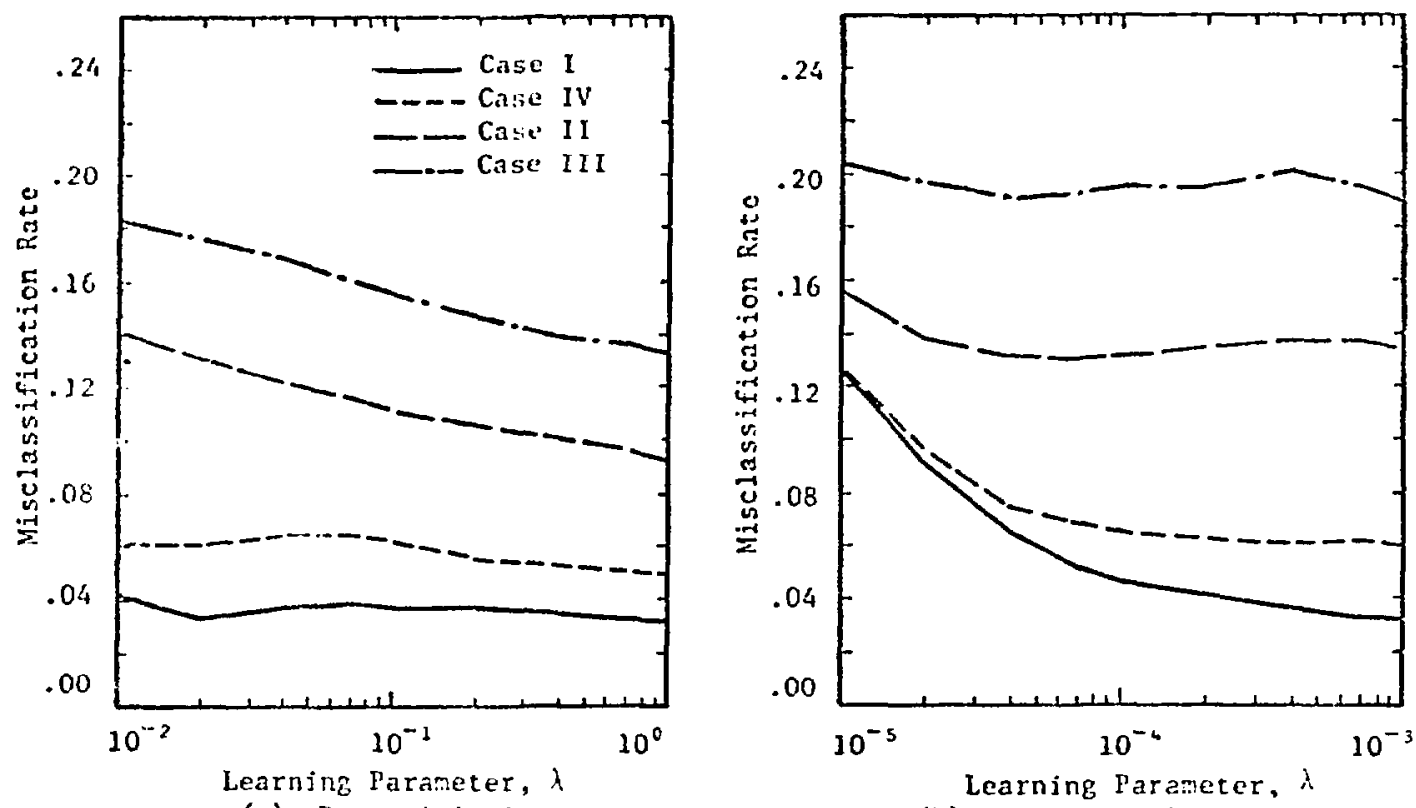

(a) Deterainistic

(b) Statistical, Loss $=\mathrm{d}$

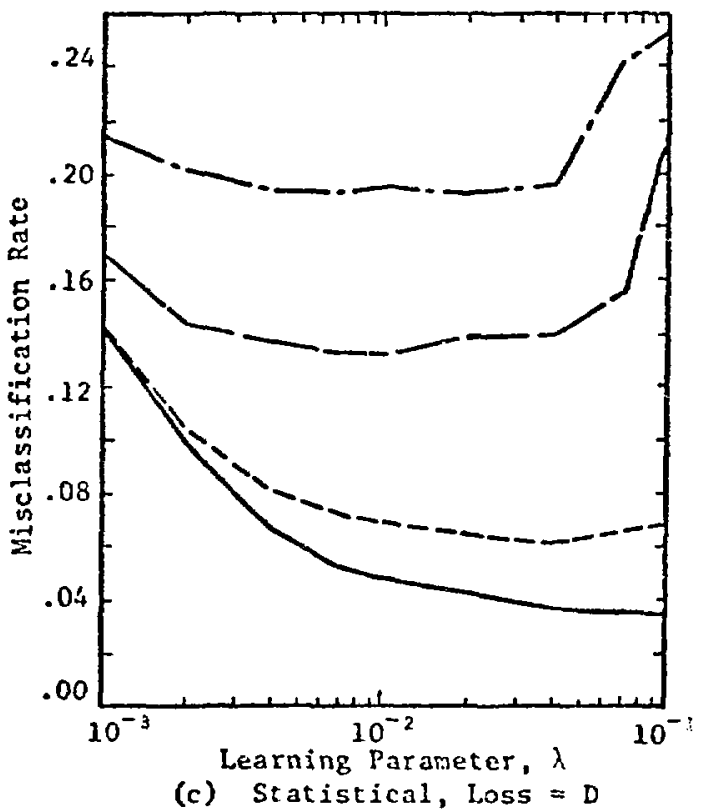

Figure 4.29 Misclassification Rate Vs. $\lambda$ for Four Different Multi-Region Problens 


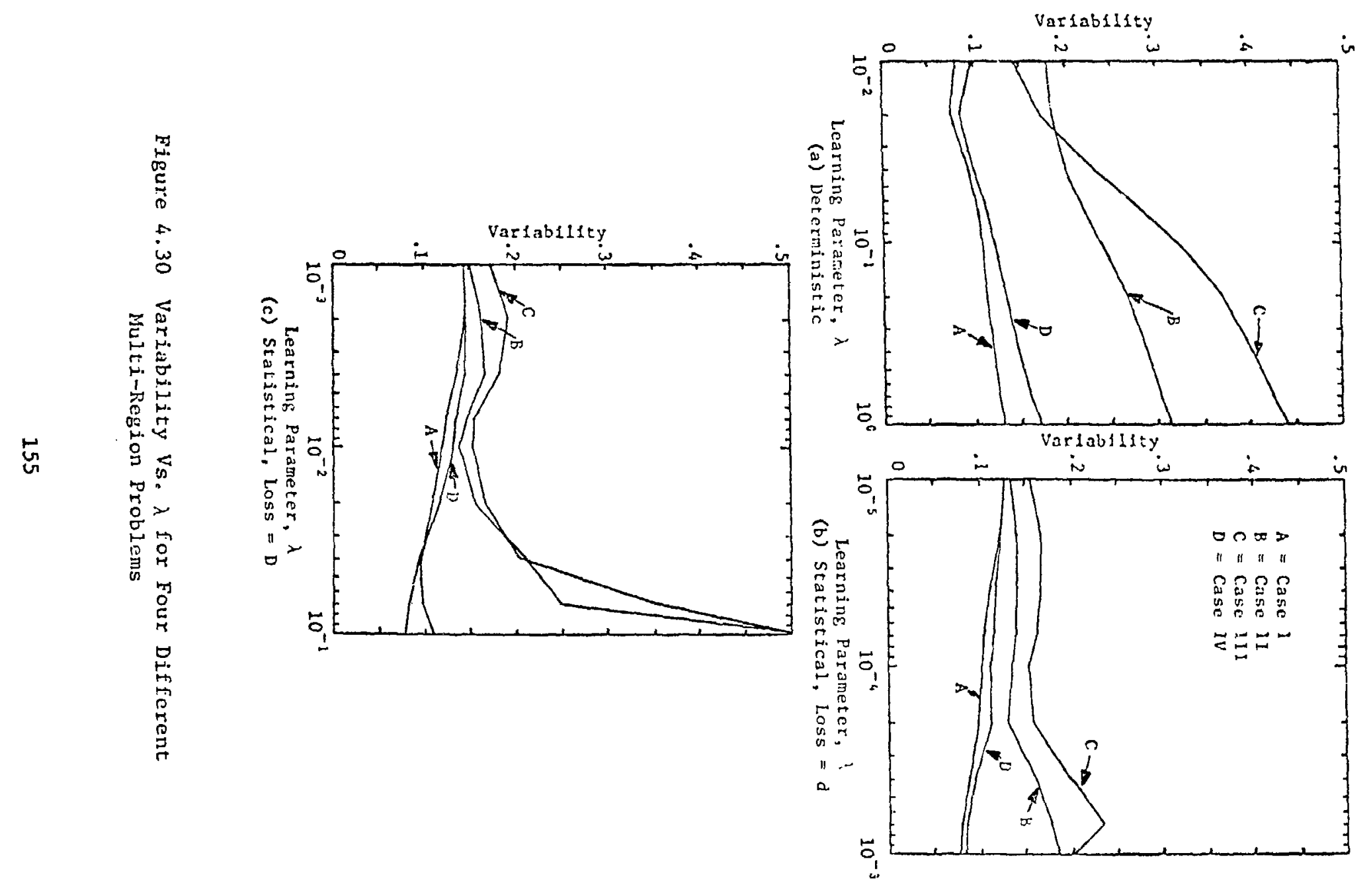


small improvement for Cases $I$ and IV. Of the two statistical techniques the loss function using d results in slightly better performance a1though the difference is small.

Because a pattern classifier must be able to operate on a large range of problems without alteration, a single $\lambda$ must be chosen for each classifier. For the purpose of this investigation, $\lambda$ 's of $10^{-1}, 10^{-4}$, and $10^{-2}$ will be used for the deterministic, statistical (loss =d), and statistical (1oss =D) classifiers respectively. Although this selection has not been optimized, these values are at least a reasonable compromise as is seen from Figures 4.29 and 4.30 for decreasing the error while keeping the increase in variability down.

For these values of $\lambda$, the performance for the first 100 particles is shown in Figures 4.31 and 4.32. It is interesting to note that in all cases the deterministic classifier leads to a smaller misclassification rate. Because the prototypes in these problems are presented in the order that they occur (i.e., for a single source particle $\mathrm{x}_{1}<\mathrm{x}_{2}<\mathrm{x}_{3} \ldots<\mathrm{x}_{\mathrm{N}}$, where $\mathrm{N}$ is the number of collisions) and because the deterministic classifier responds only to a single prototype at a time, it has an advantage over the statistical technique. For example, consider the following prototypes and a current $s_{i}=100$ :

$$
\begin{aligned}
i & =\ldots 9,10,11,12,13,14,15 \ldots \\
x_{i} & =\ldots 75,83,91,97,109,114,119 \ldots \\
\text { Class } & =\ldots c_{1}, c_{2}, c_{2}, c_{2}, c_{2}, c_{2}, c_{2} \ldots
\end{aligned}
$$




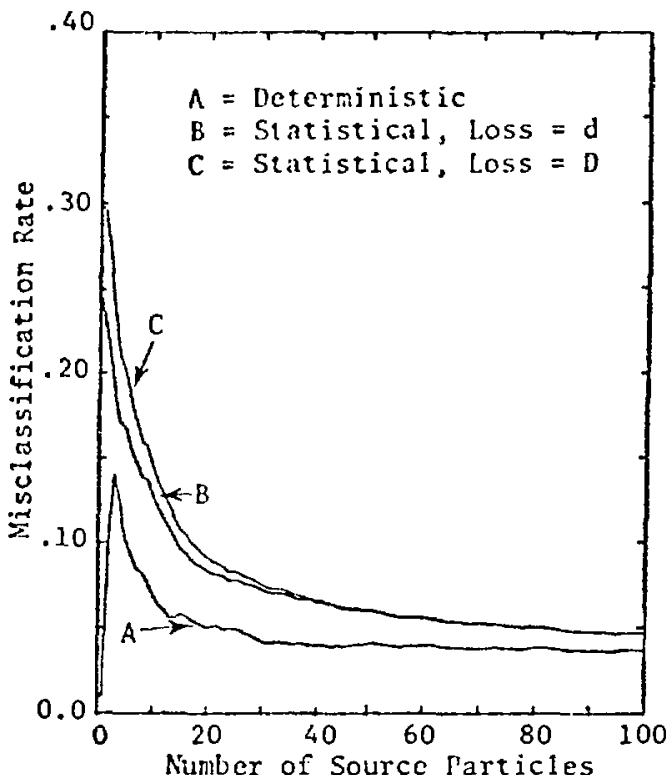

(a) Case I

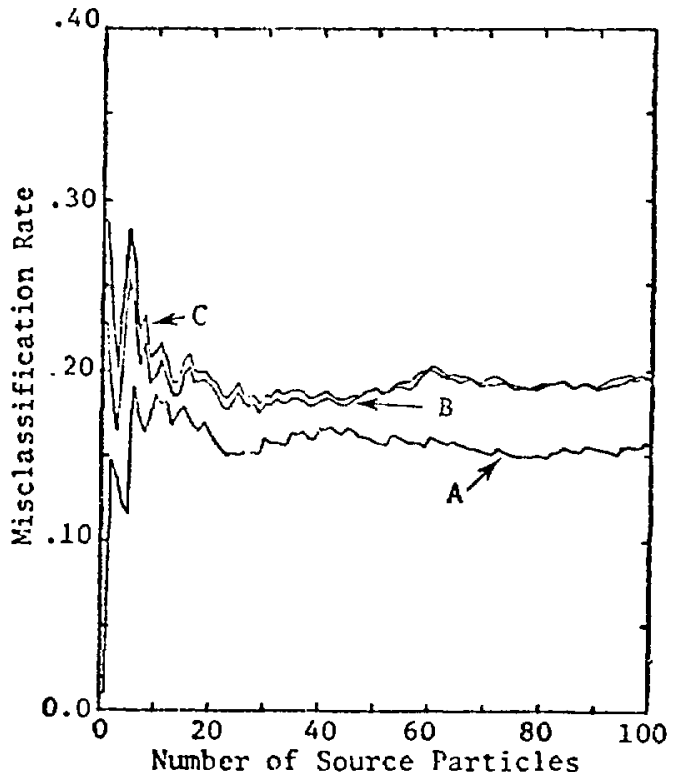

(c) Case III

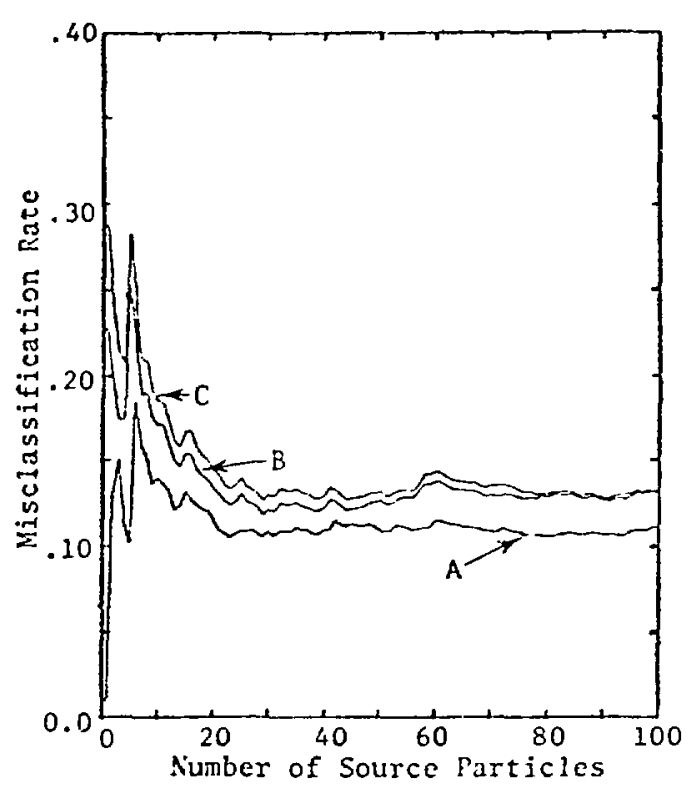

(b) Case II

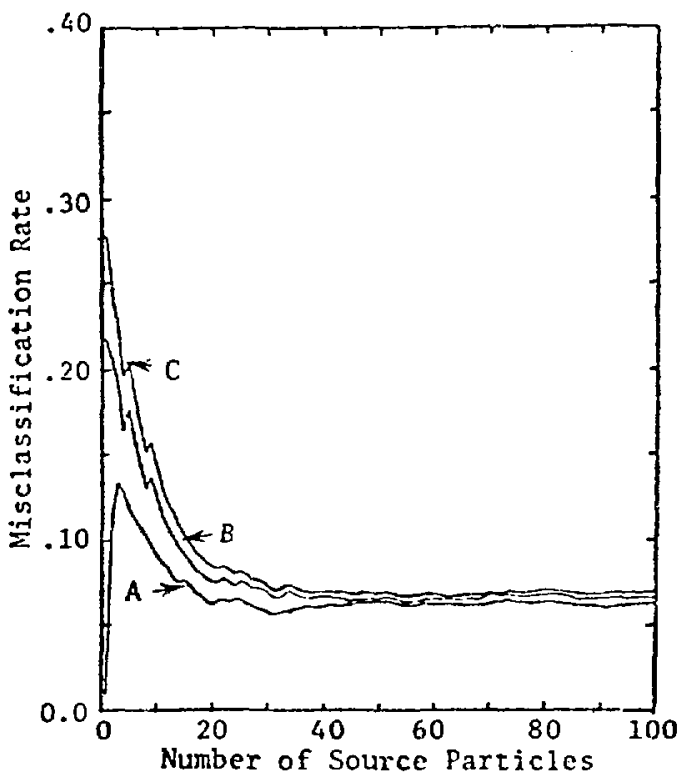

(d) Case IV

Figure 4.31 Misclassification Rate Vs. Nurber of Scurce particles for Four Different Multi-Region Problems 


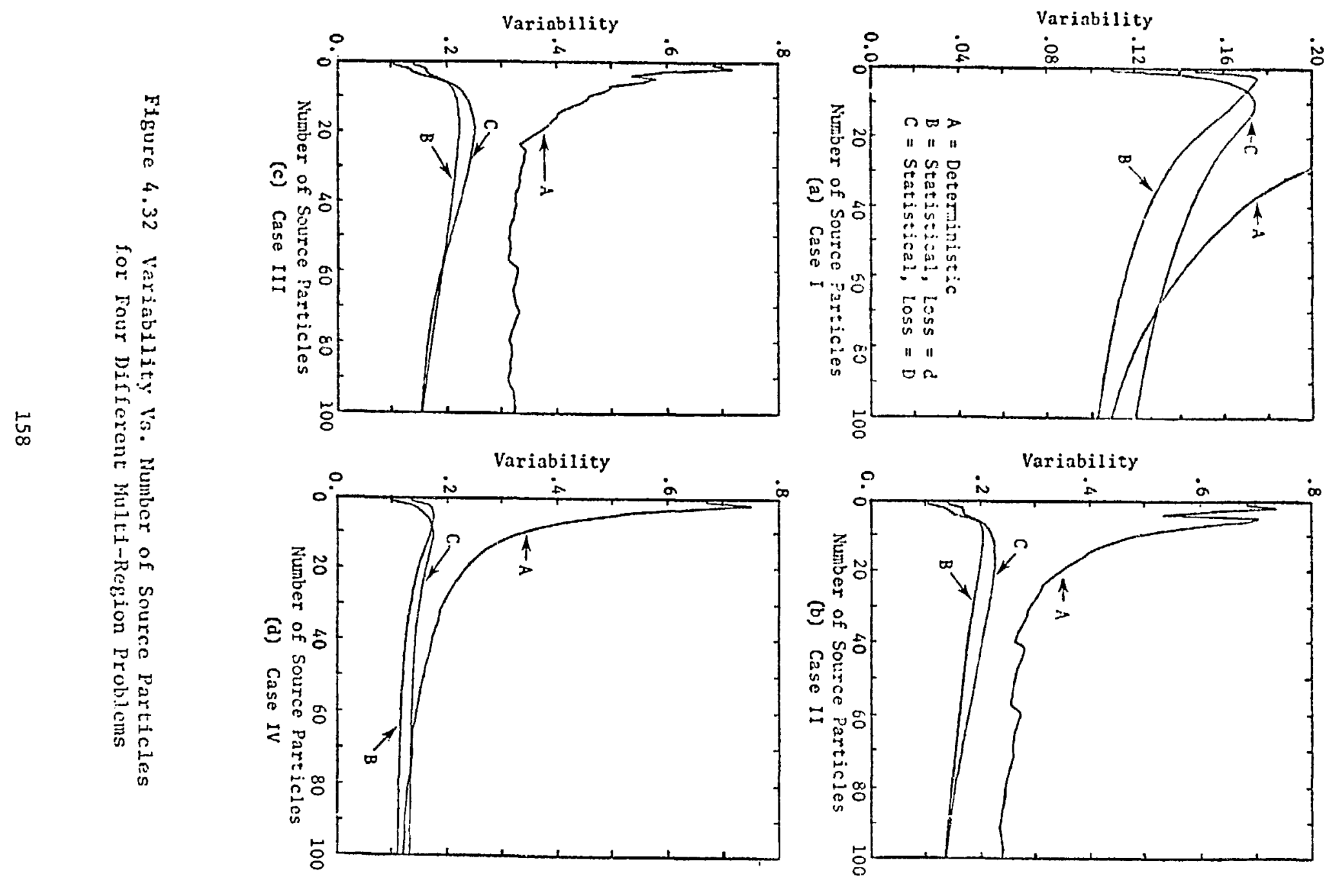


If $\lambda=1$, and $x_{10}$ is presented to the deterministic classifier, the new $\mathrm{S}_{j+1}=83$ which in turn makes $\mathrm{x}_{11}$ and $\mathrm{x}_{12}$ correctly classified. For $\lambda<1$, the deterministic classifier still has this effect to some degree. The statistical classifier may or may not decrease $S_{\mathbf{j}}$ in this case depending on the value and sign of $\mathrm{T}_{1}$ and $\mathrm{T}_{2}$ (see Equations 4.14 and 4.15). Although this appears to be an advantage of the deterministic classifier, it may actually be a disadvantage since the objective is to minimize the number of misclassified prototypes according to their average importances and not the importance encountered for each prototype. Thus the misclassification rate shown in Figure 4.29 may be a "false misclassification" rate since this is the misclassification rate as seen by observing prototypes one at a type instead of looking at the average importance of each prototype. This phenomenon is of ten referred to as "learning from a teacher who makes mistakes" since the classifier must use prototypes which are misclassified part of the time. In summary, the deterministic classifier performs better for non-overlapping or slightly overlapping classes. However, as the overlapping increases statistical techniques become increasingly more attractive because of their lower variability.

\section{4) Distance and Angle, A Two-Dimensil ial Problem}

In previous sections of this chapter the pattern space has consisted of one dimension--distance. In this section the Monte Carlo. problem consists of the multi-region problem shown in Figure 4.17 except that after each collision, a scattering angle is calculated 
(see Figure 4.33). For the purposes of this research, scattering is assumed isotropic in the laboratory system. The FORTRAX coding used for the calculations in this section is shown in Appendix ll.

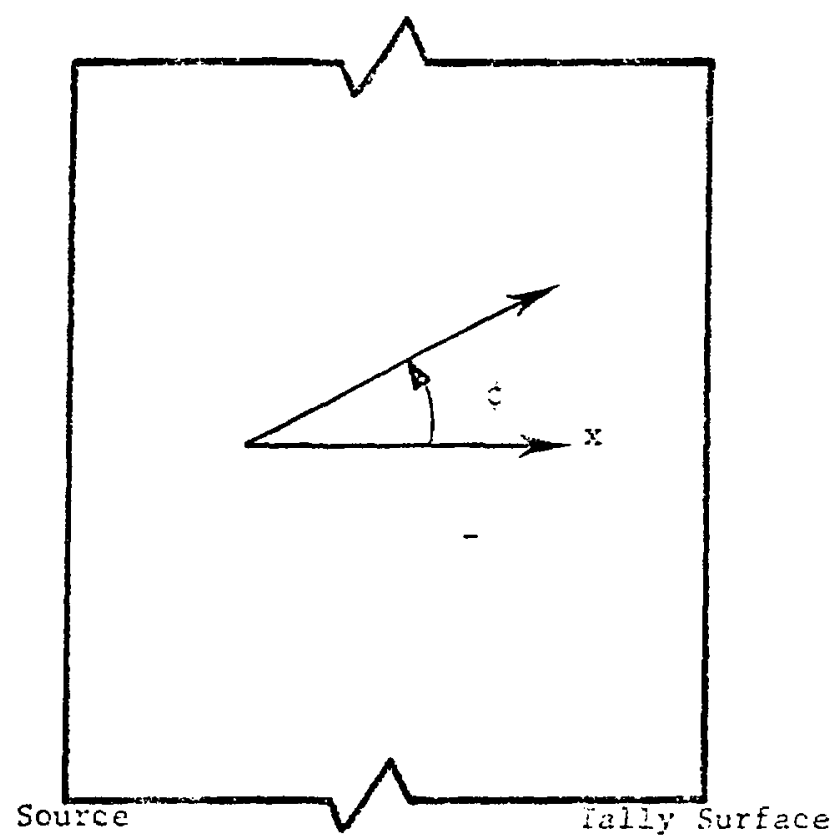

Figure 4.33 Fite Tho Di-ensional tonte Carlo Problem 
Because the number of regions in the slab does not affect the structure of the pattern classifier, a single region will be used with $\Sigma_{t}=.5$ and $\Sigma_{s}=.4$. A slab thickness of five mean-free-paths $(L=10)$ is chosen for this example in order to keep computer time at a minimum while still maintaining the characteristics of a two-dimensional problem.

A significant difference between this problem and the previous one-dimensiona 1 problems for which $L=200$ is the number of prototypes (equal to the number of collisions). The one-dimensional problen creates 100 prototypes per source particle, whereas the two-dimensional problem of this section produced only 11 . Consequently the two-dimensional problem investigated in this section requires more source particles to learn a splitting surface. An important characteristic of the two-dinensional problem is that particles are allowed to escape from the system without contributing to the tally. As a result the distribution of track importances consists of a distribution of importances created by non-zero tallies plus a number of track importances equal to zero. This is a common feature of the majority of Monte Carlo problems. Because of this, one wishes to locate splitting surfaces so as to get particles from regions of zero importance into regions of non-zero importance. Therefore, the $\bar{I}$ described in the introduction to this chapter is equal to zero. 
In the sample problem of this section approximately $59 \%$ of all tracks have zero importance. The distribution of the remaining $41 \%$ is shown in Figure 4.34a. The probability distribution of prototypes, $p(Y)$, as a function of $x$ is shown in Figure $4.34 b$ for three different contours through $x-\phi$ space and in Figure $4.34 \mathrm{c}$ as a function of $\phi$. The decreasing probabilicy of procotypes with increasing $x$ is a result of fewer particles penetrating the slab and thus fewer prototypes occur deep within the slab. The flat distribution with respect to $\cos \phi$ results from isotropic scattering.

Given the feature vector $Y=[x \phi]$, the probability that $Y$ belongs to class $C_{i}$ is given by $p\left(C_{i} \mid Y\right)$, (see Section 3.3). Plots of $p\left(C_{i} \mid Y\right)$ for the same contours used in Figure $4.34 \mathrm{~b}$ and $\mathrm{c}$ are shown in Figure 4.35. The symmetry of the class distributions is due to isotropic scattering and the single material region. The intersections of the distributions define the decision surface. A particle with coordinates on this decision surface has an equal probability of being tallied or escaping. Un1ike the one-dimensional problem the probability densities of each class, $p\left(Y \mid C_{i}\right)$ are not proportional to $p\left(C_{i} \mid Y\right)$ since $p(Y)$ is not constant. The misclassification rate due to the overlapping of $p\left(C_{i} \mid Y\right)$ is $29 \%$ (1.e., $E r=29$ where $\operatorname{Er}$ is defined by Equation 4.5). In Section 4.2 .3 normalization was achieved by multiplying the feature vector $\mathrm{Y}$ by $1 / \mathrm{L}$ and altering the adjustment algorithms accordingly. This is not possibif for two-dimensional pattern space since both variables $\phi$ and $x$ must be normed with respect to each other. This is 


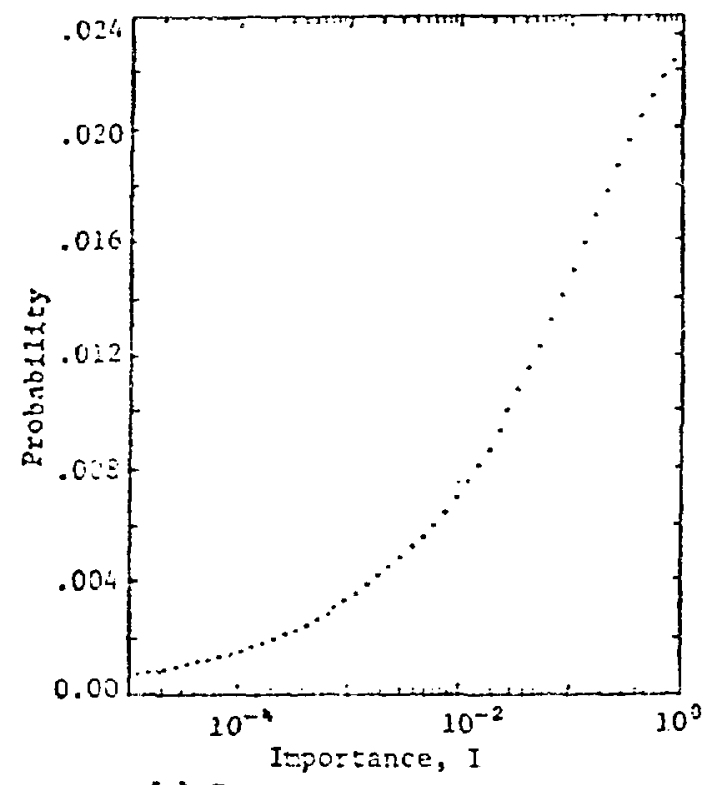

(a) Inportance Discriouticn

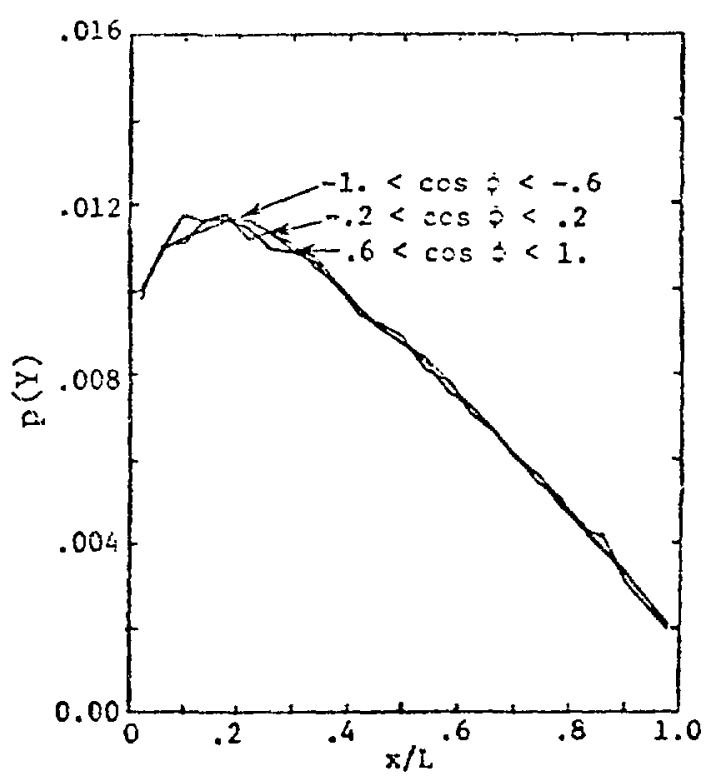

(b) $p(Y)$ vs. $x$

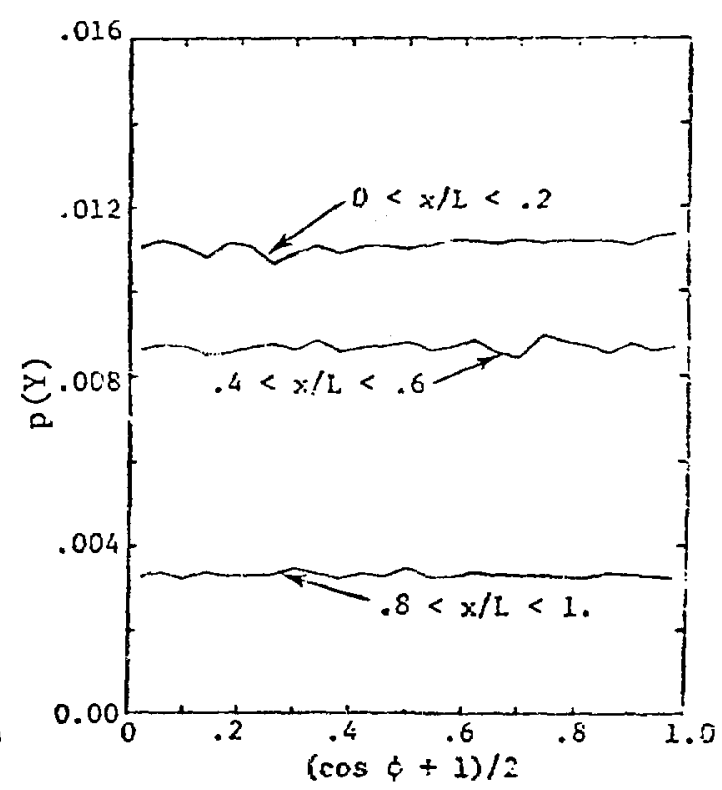

(c) $p(Y)$ vs. G

Figure 4.34 Irportance and prototype Distributions for the Tro Dimensional Monte Carlo Problem 


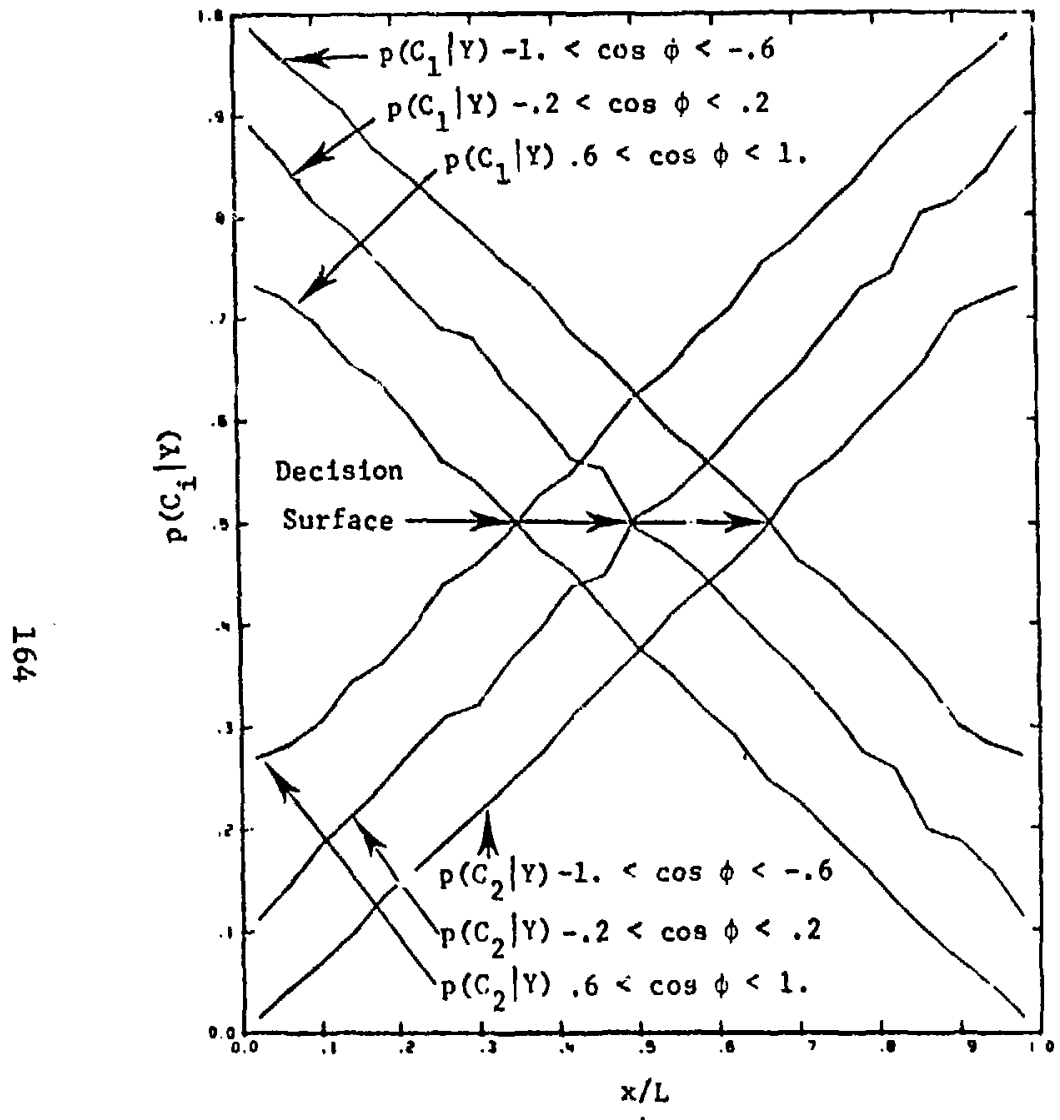

(a) $p\left(C_{1} \mid Y\right)$ Vs. $x$

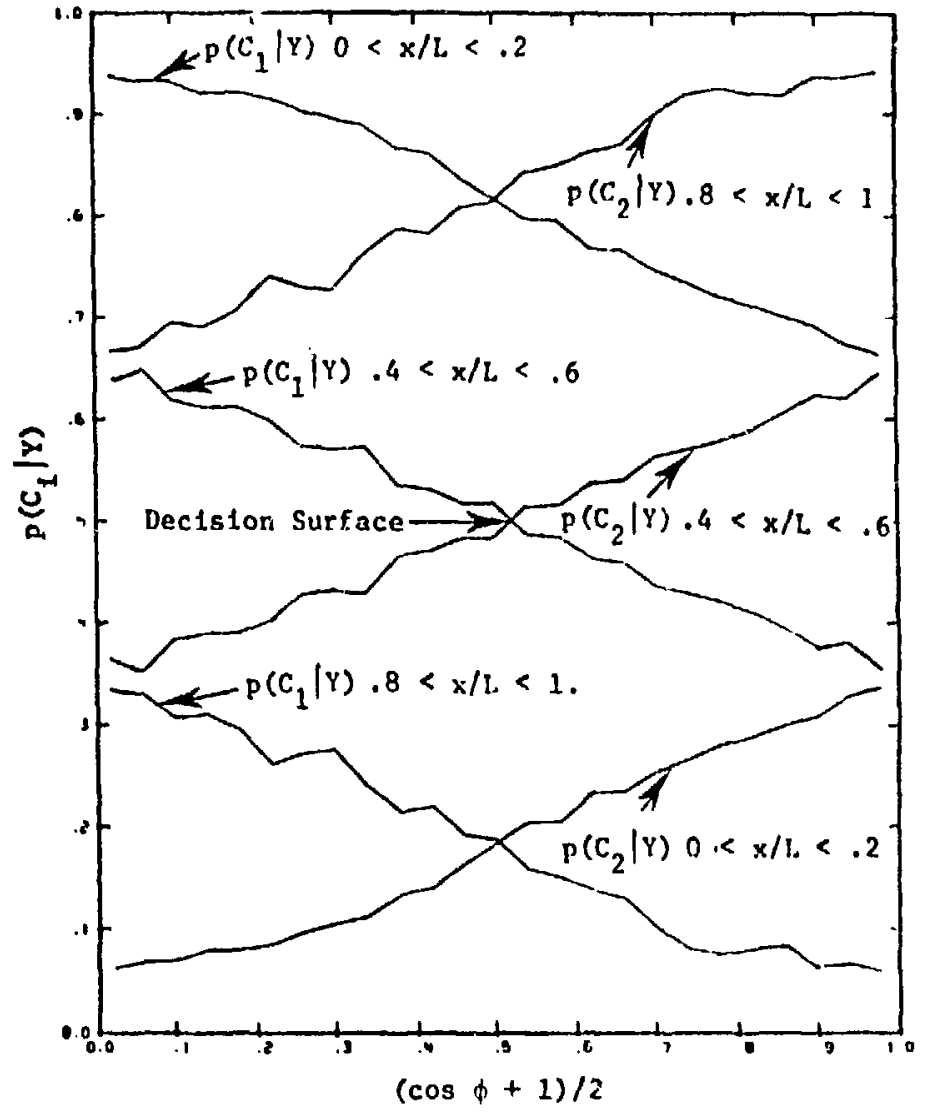

(b) $p\left(c_{1} \mid Y\right)$ vs.

Figure 4.35 Class Distributions for the Two Dimensiona1 Monte Carlo Problem 
done by normalizing both angle and distance to one. Thus, pattern space iss transformed to feature space as shown by Equation 4.22 .

$$
X=\left[\begin{array}{l}
x_{1} \\
x_{2}
\end{array}\right]=\left[\begin{array}{l}
x \\
\phi
\end{array}\right] \quad Y=\left[\begin{array}{l}
y_{1} \\
y_{2}
\end{array}\right]=\left[\begin{array}{c}
x / L \\
(\cos \phi+1) / 2
\end{array}\right]
$$

In the one-dimensional slab problem the performance of the classifier is measured in terms of the misclassification rate and the variability of the decision surface. The misclassification rate wil be used unchanged; however, the variability of the decision surface (a line in two dimensions) is given by

$$
\text { Variability }=\frac{\sigma_{x}{ }^{\sigma} \phi}{\sqrt{\bar{\phi}^{2}+\bar{x}^{2}}}
$$

where $\bar{\phi}=$ the mean value of the decision surface with the $y_{2}$ $[(\cos \phi+1) / 2]$ axis $\bar{x}=$ the mean value of the decision surface with the $y_{1}(x / L)$ axis

$\sigma_{x}=$ standard deviation of the mean $\bar{x}$ $\sigma_{\phi}=$ standard deviation of the mean $\bar{\phi}$ 
In additiun to investigating learning parameter influence, this section studies the effects of initial conditions as was done in Section 4.3.1 for the ome-dinensional case. For one-dimensional feature space the initial condition consists of a single variable $s_{1}=-w_{2} / w_{1}$. The choice of an initial $\because_{1}$ ras found to have no effect provided the proper $\lambda$ were selected. Thus by keeping $\lambda=$ constant, an juitial $v_{1}=1$ could be used without loss of generality. Figure 4.36 illustrates the decision surface for normalized two-dirensional feature space. From this figure it is seen that two pararjeters are required to specify the decision surface, $-w_{3} / w_{2}$ and $-w_{3} / w_{1}$. A third parameter (in this case let it be $w_{1}$ ) determines the slope of the discrininant function and like the one-dimensional case can be set equal to 1 provided that $\lambda$

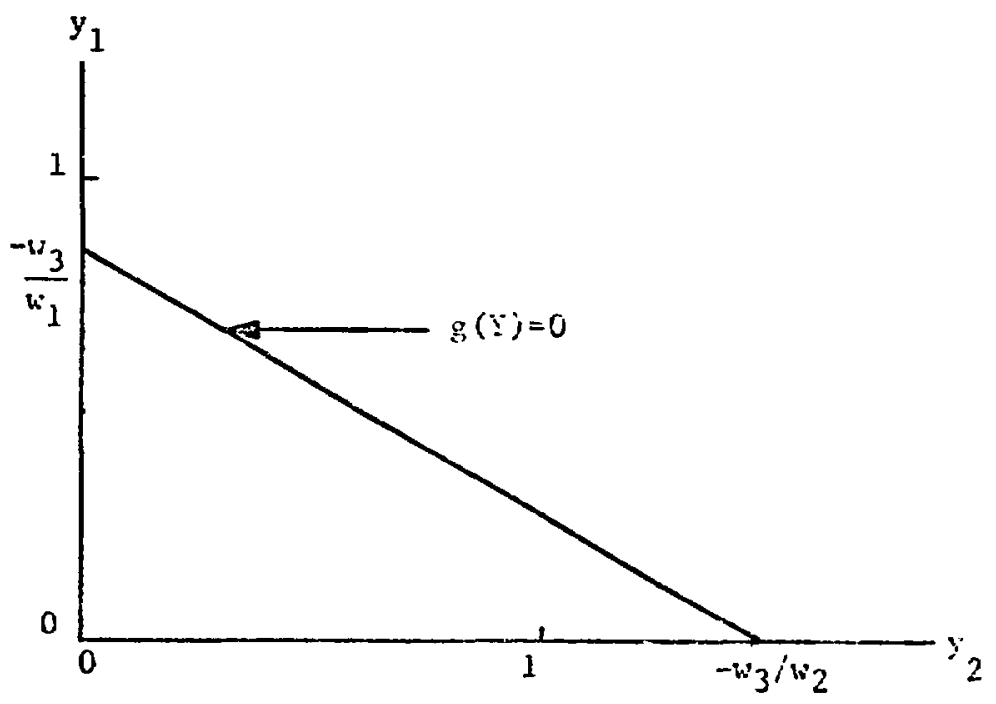

Figure 4.36 Decision Surface for the Two Dimensional problem 
remains constant. A simplifying assumption made only for the purposes of this research is to use $w_{2}=w_{1}=1.0$ which results in a decision surface (line) oriented at $45^{\circ}$ to the $y_{1}$ and $y_{2}$ axes. Using this assumption, the initial conditions consist of specifying $w_{3}$, where $-w_{3}$ is the $y_{1}$ and $y_{2}$ intercept.

It was demonstrated in section 4.3.1 that an initial decision suriace near the origin leads to decreased performance and it was recomended that this problea be alleviated by changing the feature $x$ to $\mathrm{L}-\mathrm{x}$. This situation also occurs in the two-dimensional proble:n. Therefore, several runs will be rade using $y_{1}=1-x / L$ and $y_{2}=1-(\cos \phi+1) / 2$. For these runs $w_{1}=w_{2}=-1.0$ and $w_{3}$ will be chosen to correspond to an equivalent $w_{3}$ in $x / L,(\cos \theta+1) / 2$ space. To illustrate this feature conversion two equivalent decision surfaces are shown in Figure 4.37. In the following sections a negative value of $w_{3}$ implies $v_{1}=w_{2}=1$ (as in Figure $4.3 \%$ a), a positive $w_{3}$ implies $w_{1}=w_{2}=-1$ (as in Figure $4.37 b$ ).

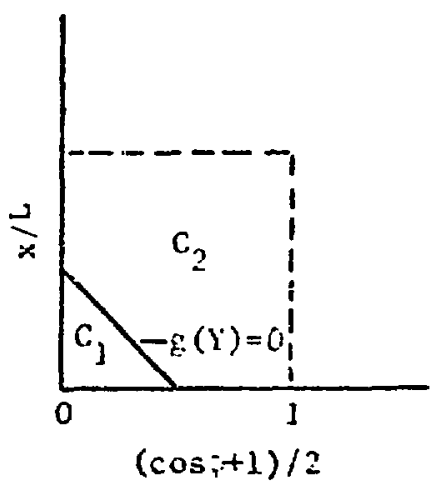

(a) $w_{1}=w_{2}=1, w_{3}=-.5$

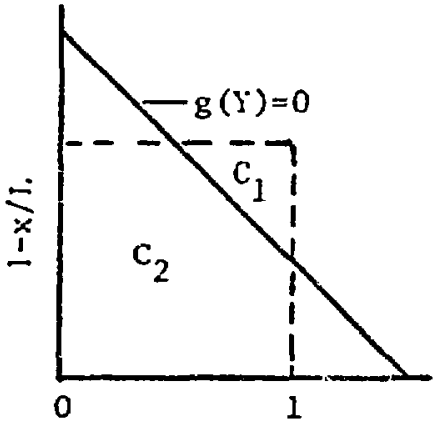

$1-\left(\cos ^{4}+1\right) / 2$

(i) $w_{1}=w_{2}=-1, w_{3}=1.5$

Figure 4.37 Feature Vector Conversion 


\subsubsection{Deterministic Classifier}

The algoritha for weight adjustment is the same as given by Equation 4.4 except that the diaension of the vectors $W$ and $Y \stackrel{\hat{i}}{*}$ is increased from two to three. The FORTRAN coding of this classifier is shown in Appendix h. The effect of the learning parameter, $\lambda$ is illustrated in Figures $4.38 \mathrm{a}$ and $4.38 \mathrm{c}$ for several di ferent initial conditions after 5000 source particles. The behavior of the classizier as a function of $\lambda$ is similar to that illustrated in Figures 4.29 and 4.30 for the problems in which the class distributions have a large arcunt of overlap (Cases II and III). The misclassification rate drops with increasing $\lambda$ while the variability increases. The fact that the aisclassification rate drops belo\% that due to overlapping classes (29\%) indicates the effect of "false risclassification" described in Secticr. 4.3.2.

The misclassification rate atd variability for runs started near the origin $\left(w_{3}=-.25,-.05\right)$ are higher than the other runs (see Figures $4.39 a$ and $4.40 a)$. When feature space is converted $\left(w_{3}=1.75\right.$, 1.95) the same prototypes lead to much better performance.

\subsubsection{Statistical Classifier}

The correction vector for the two-dimensional problem with a loss function equal to $d$ is given by 


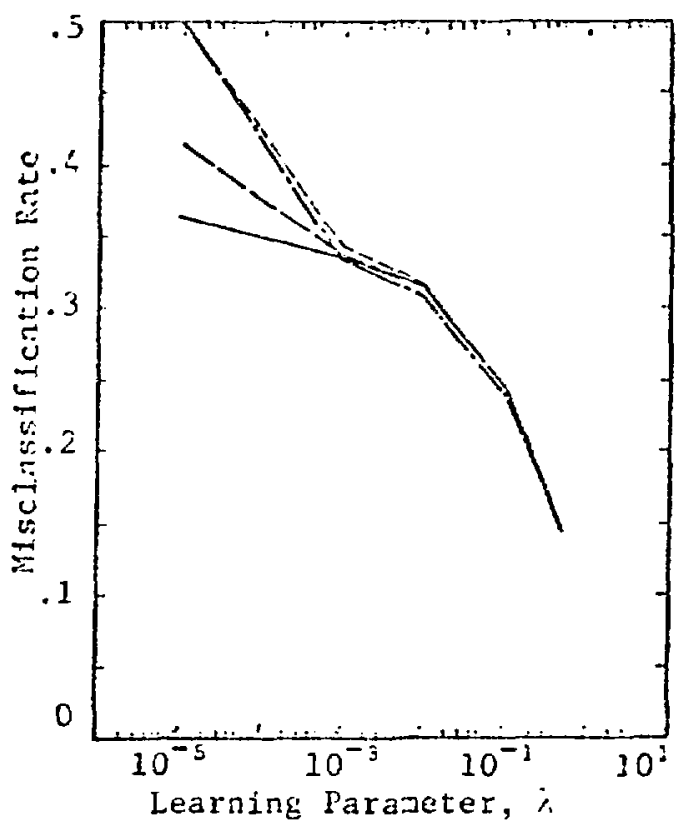

(a) Misclassification Rate (Deterministic)

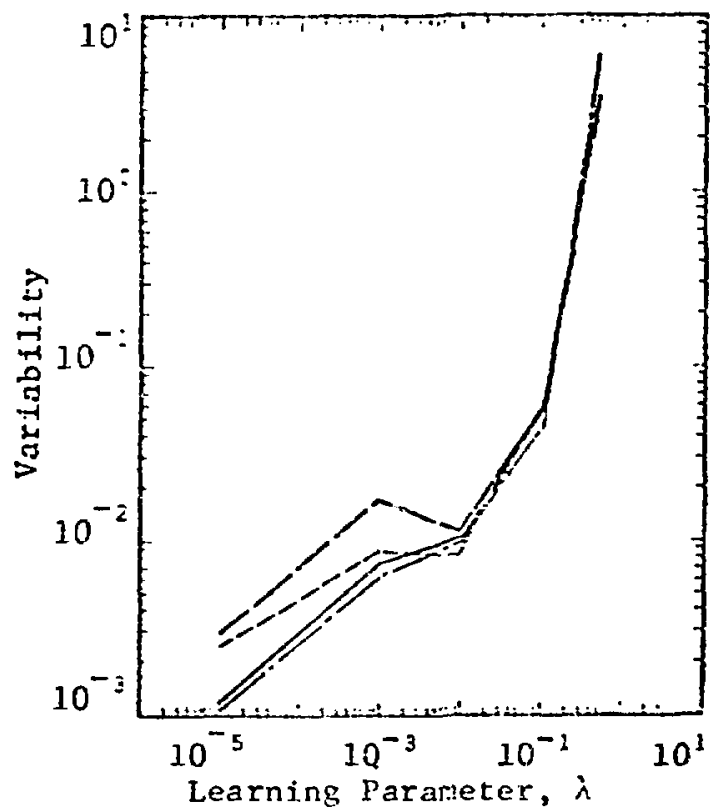

(c) Variability, Deterministic

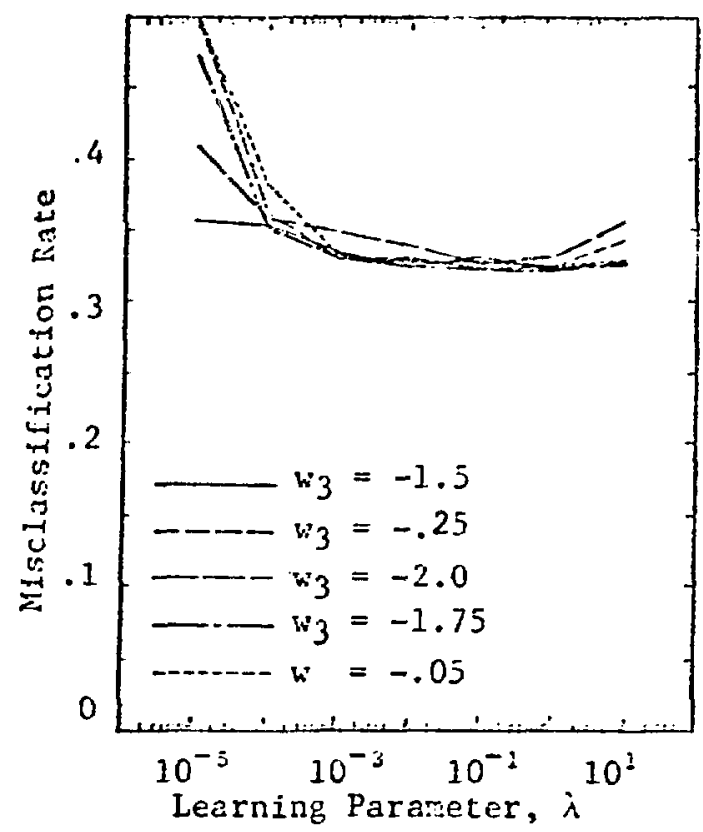

(b) Misclassification Rate (Statistical)

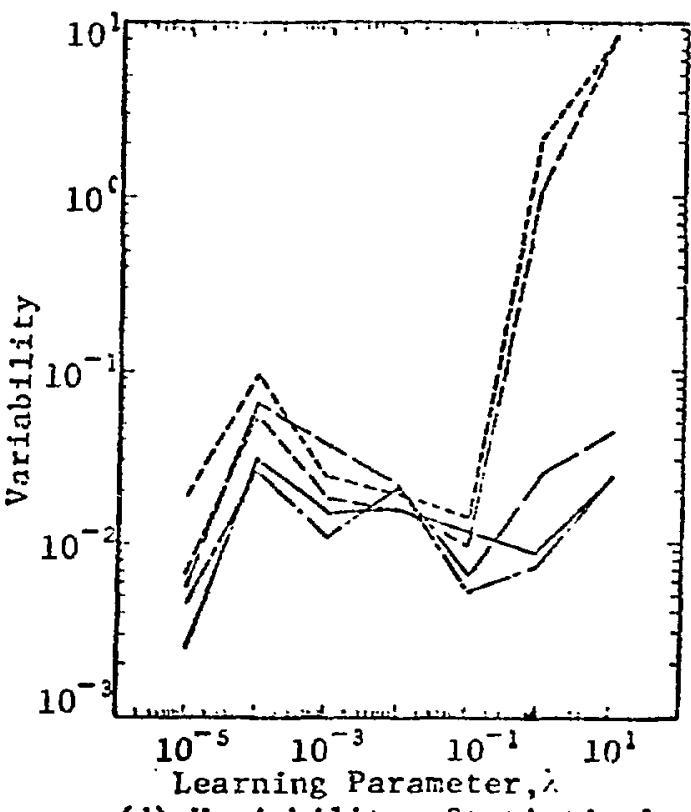

(d) Variability, Statistical

Figure 4.38 Performance V's. $\lambda$ for the Two-Dinensional Froblem 


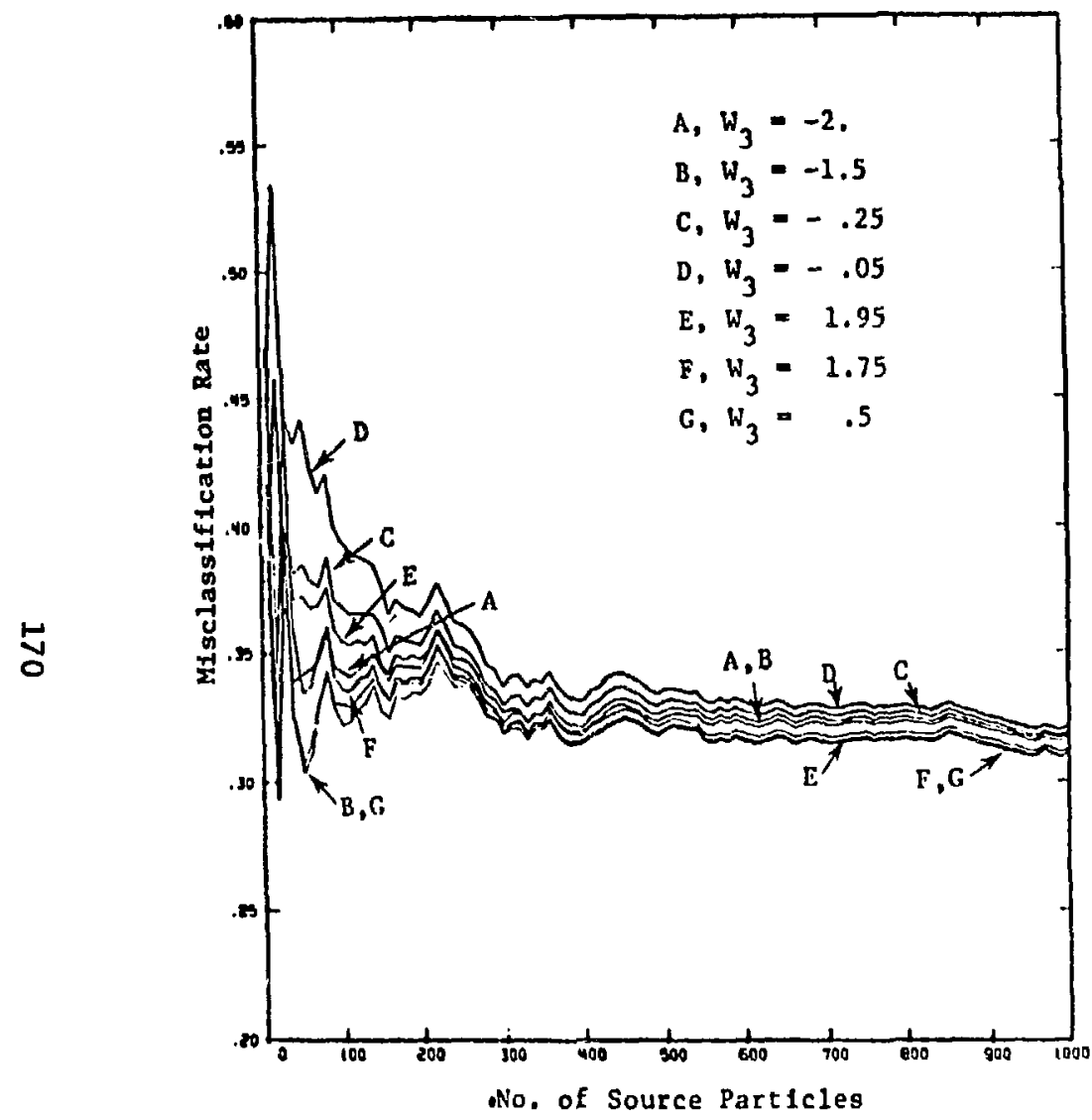

(a) Deterministic

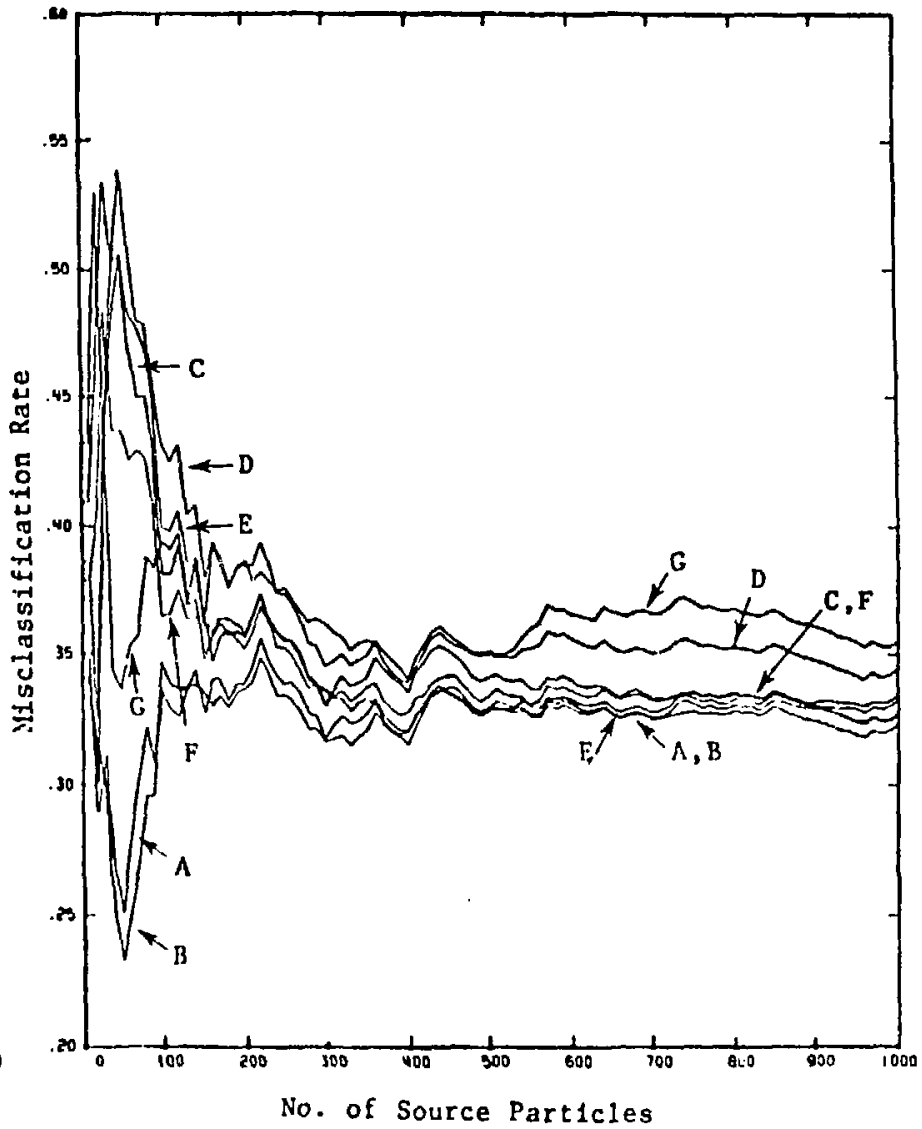

(b) Stat1st1ca:

Figure 4.39 Misclassification Rate Vs. Number of Source Particles for the Two Dimensional Problem 


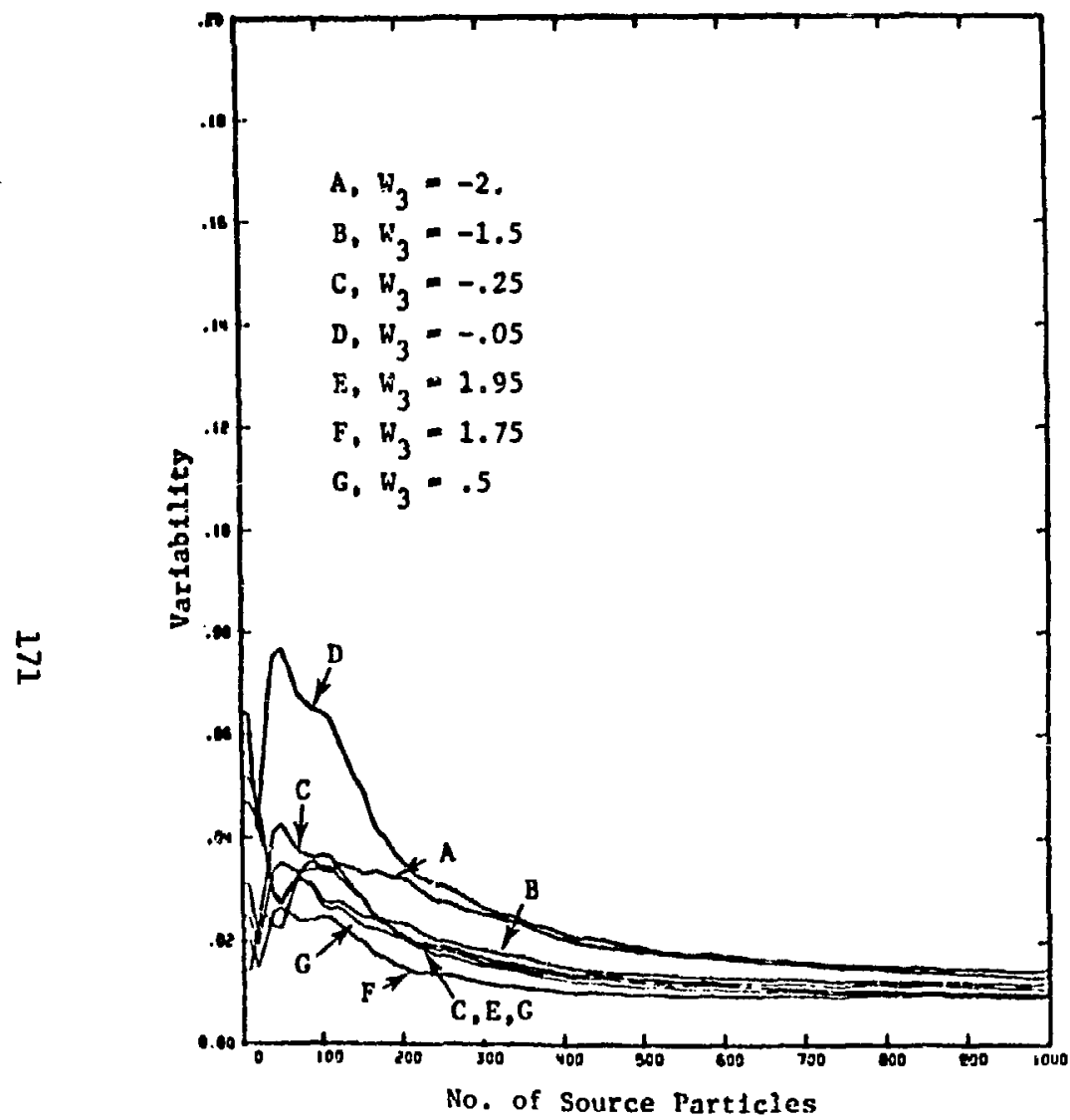

(a) Deterministic

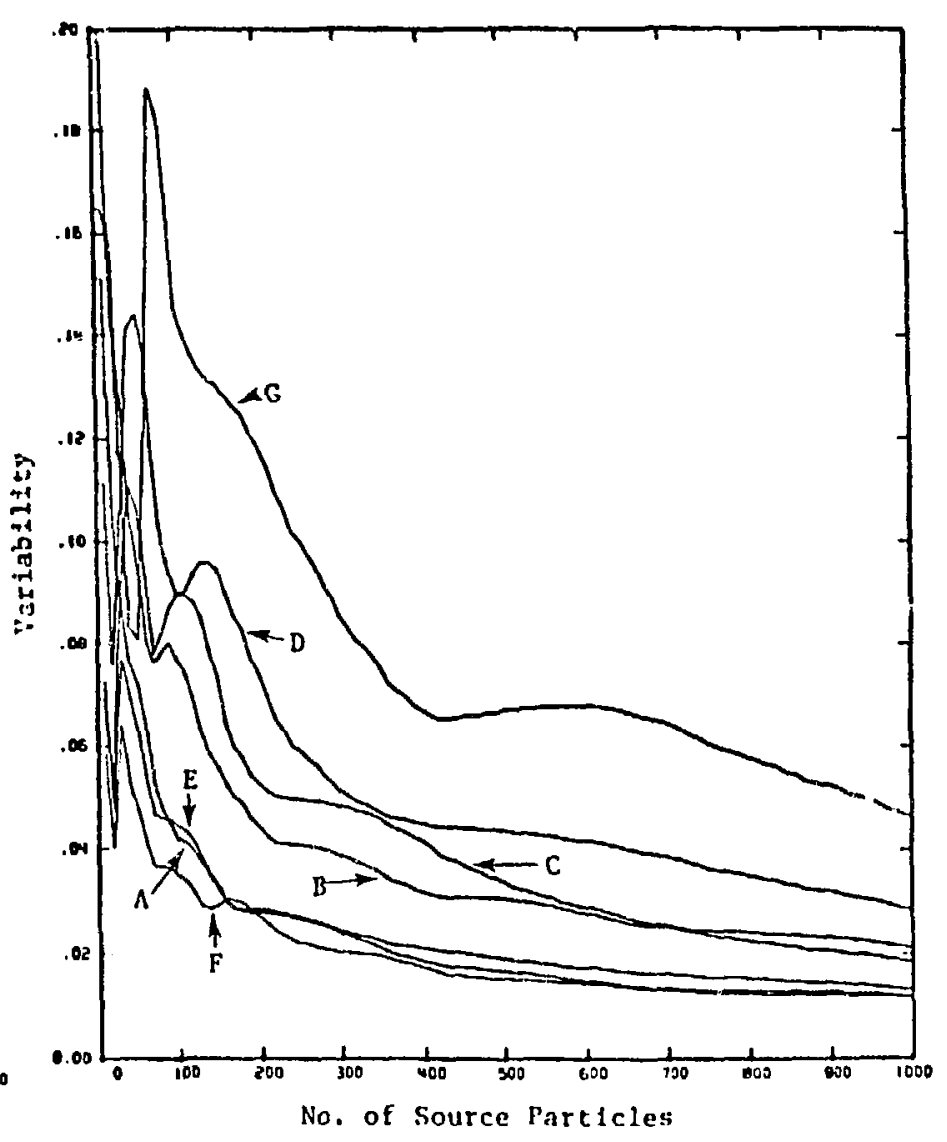

(b) Statist lcal

Figure 4.40 Variability Vs. Number of Source Particles for the Two Dimensional Problem 


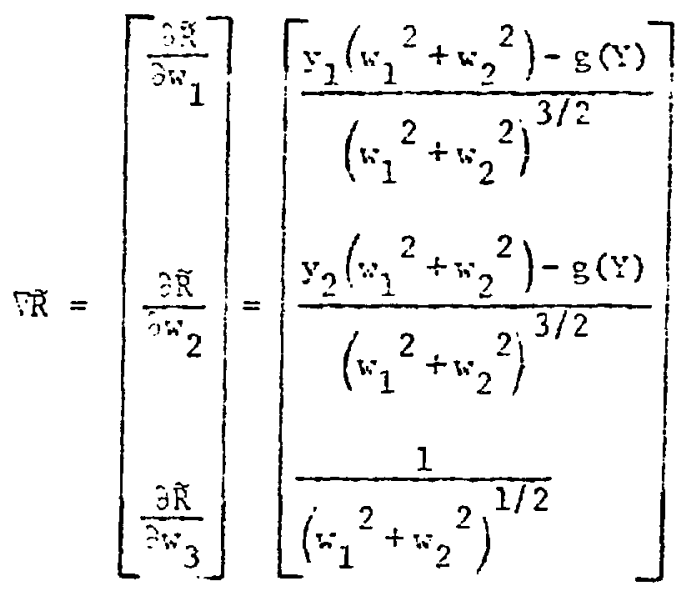

The FORTRAN coding for the statistical classifier is given in Appendix I. The risclassification rate aiter 5000 particles is relatively flat for $\lambda$ 's between $10^{-3}$ and 1 (see Figure 4.38u); however, the variability has a ninimer at $\lambda=10^{-1}$. Lnlike the deterministic classifier, normalization affects the optims $\therefore$. The aisclassification rate (see Figure 4.395) is more spread than the deterninistic classifier due to the sensitivity of the statistical classifier toaninitial decision surface near the origin. The variability is also rore spread (see Figure 4.40b) since initial surfaces nearel the origin $\left(w_{3}=-.25,-.05, .5\right)$ performed considerabiy worse than thei: councerparts awa frod the origin $\left(w_{3}=1.75,1.95,-1.5\right)$. Thus the statistical classifier behaves the same as previously illustrated in one disension.

\subsection{3) Sumnary}

Aside from the norzalization procedure the two-dimensional problem behaves sinilar to the one dimensional cave. Once again the classi- 
flers perform better when not operating near the origin and, as has been shown, inversion of the feature vector can be used to alleviate this problem.

The time spent by the two techniques can be compared by using Equation 4.9 and the timing parameters given in Table 4.7. The time required for normalization of features is not included in this data since it is a feature selection process and will be treated in Chapter $v$. The time to determine classification, $t_{A}$, is unchanged since it does not involve the dimensionality of $Y$. The increase in $t_{I}$ is due to the additional terms necessary to calculated $g(Y)$. The weight adjustment times increase by $100 \%$ for the statistical case and by $66 \%$ for the deterministic case: The example times given below are for 300 source particles (3,590 prototypes), an initial $w_{3}=1.95$ (see Figures 4.39 and 4.40, runs E) and no buffer zones.

$\begin{array}{lcr} & \frac{\text { Statistical }}{21.5 \times 10^{-6}} & \text { Deterministic } \\ { }^{C} & 1.7 \times 10^{-6} & 5.8 \times 10^{-6} \\ t_{L} & 4.1 \times 1.0^{-6} & 1.7 \times 10^{-6} \\ t_{A} & 4.1 \times 10^{-6} \\ \text { Table 4.7 } & \text { Timing Parameters for the Two } \\ & \text { Dimensional Problem }\end{array}$


Deterministic $\left(\mathrm{f}_{\mathrm{C}}=.327\right)$

$$
T=1.47 \times 10^{-2}+.61 \times 10^{-2}+.68 \times 10^{-2}=2.76 \times 10^{-2}
$$

Statistical $\left(E_{C}=.330\right)$

$$
T=1.47 \times 10^{-2}+.61 \times 10^{-2}+2.55 \times 10^{-2}=4.63 \times 10^{-2}
$$

Unlike the one dimensional case of Section 4.2 .3 the difference between the two techniques is significant. This difference is due primarily to the increase in the amount of class overlap (from $4 \%$ to the 1-D case to $29 \%$ for 2-D) which causes the effect of $t_{C}$ to be more pronounced in the two-dimensional case.

\section{$4.5 \quad$ Sumary}

In this chapter statistical and deterministic classifiers have been used to learn splitting surfaces for several different Monte Carlo problems. The conclusions drawn from these numerical experiments are listed below for each topic.

Slab Thickness and Class overlapping. It was found in section 4.2 that the smaller the distance variable (in this case slab thickness) is in terms of mean free paths, the greater is the amount of class overlapping. Because class overlapping decreases classifier performance, geometries of many mean free paths are easier problens as far as pattern recognition is concerned. 
Buffer Zones. Buffer zones decrease the misclassification rate of the classifier by removing prototypes which are very close to class boundaries (according to the teacher). However, if prototypes falling within the buffer zone are not used as prototypes, the variability can be increased since the variability is proportional to the inverse of the number of prototypes. Therefore, prototypes within the buffer zone should be considered as correctly classified prototypes. A further benefit of buffer zones is that they decrease the amount of computer time necessary for pattern recognition.

Loss Functions. Although there was no great difference in performance for the loss functions used, loss=d appears to be the most attractive since it is the simplest computationally.

Learning ?arameter, $\lambda$. It is desirable to use a single $\lambda$ for a11 problems since this greatly simplifies the classification process. As has been seen in previous sections a range of $\lambda$ does exist for both statistical and deterministic classifiers over which the performance is relatively constant. If a $\lambda$ below this range is used, the convergence of the classifier is slowed down requiring additional time for the misclassification rate to decrease sufficiently. A $\lambda$ above this range causes the variability to increase although it may actually decrease the misclassification rate. This range is uafected by normalization for the deterministic classifier but is strongly affected for the statistical classifier. The following appear to be reasonable values of $\lambda$ that are suitable for a wide range of problems. 


$$
\begin{aligned}
& \text { Determinisic } .01 \leqslant \lambda \leqslant .] \\
& \text { Statistical (normalized to } 1.0 \text { ) . } 01 \leqslant \lambda \leqslant 1
\end{aligned}
$$

Problens with a large amount of overlap require $\lambda$ 's in the lower part of this range (small overlap, the upper part). Nonte Carlu proslems affect the choice of : only because of differences in the amoun: of class overlap. Values of .05 and .1 can be used for the detersiaistic and statistical classifiers respectively without greatly penalizing performance generality.

Initial Conditions. Although both techniques are sensitive to the selection of an initial decision surface, this selection is far wore critical for the statistical classifier. The problen can be alleviated by altering the feature vector and thus changing fros the region near the origin to a region near the point $\left(y_{1}, y_{2}, \ldots y_{n}\right)$ $=(1,1, \ldots, 1)$. As vill $\vdots 2$ seen in the next chapter decision surfaces will be started at the origin; therefore, this alteration of feature space is necessary.

Computer Tire. Although programing efficiency has been overlooked in the construction of the classifiers, some conclusions can be dratm from the timing data. These values can then be used as an upper limit for the time spent in pattern classification. The time to determine prototype classification, $t_{A}$, is a major contributor to tise spent on pattern classification, amounting to about $4.1 \times 10^{-6}$ seconds per prototype. Using Tables 4.5 and 4.7 and extrapolating to feature space of $N$ dimensions results in 


$$
L_{L} \approx 1.0 \times 10^{-6}+(\therefore-1) .7 \times 10^{-6}
$$

Performing the spme extrapolation for $t_{C}$ results in

$$
\begin{aligned}
& \text { Deteministic: } t_{\mathrm{C}} \approx 3.5 \times 10^{-6}+(\mathrm{N}-1) 2.3 \times 10^{-6} \\
& \text { Statistical: } \quad t_{C} \approx 10.5 \times 10^{-6}+(N-1) 11 . \times 10^{-6}
\end{aligned}
$$

Both the one-di=ensional ani two-dimensional problems reguire about the same number of prototypes ( 3 to 4 thousand) until convergence. However, due to difference in the overlapping of class distributions, the onedimensional case converges to a aisclassification rate of $\approx 5 \%$ whereas the two-dibensional case converges to a rate of $\approx 30 \%$.

Normalization. The norralization of feature space for nultidimensional feature vectors has been accomplished by normalizing the feature space to a $1 \times 1$ coorcinate system.

Deterministic vs. Statistical. Although the statistical classifier has the advantage that it is guaranteed to converge in the presence of overlapping class distributions, this advantage is not great since the decision surface will be used prior to final convergence. The deterministic classifier appears to be the most attractive because: (1) its performance (variability and misclassification rate) is usually better than the statistical classifier (2) it uses less 
computer tine (3) its learning parameter, $\lambda$, is not afrected by normalization and (4) it is such less affected by the initial. selection of a decision surface. 
Chapter V. Discussion of General Application

Many practical considerations remain before the techniques of Chapter IV can be applied to a multi-purpose particle transport code. The purpose of this chapter is to describe and analyze these considerations. The first problem is that given the ability to recognize surfaces, what surfaces are desirable, and in what order should those surfaces be learned. Tnis problem is analyzed in Section 5.1 for a single tally, the point detector tally, and multiple-tallies.

Section 5.2 describes the problems encountered in feature selection and suggests a scheme for implementing the feature selection process in a general purpose code. Section 5.3 investigates the amount of time required for pattern recognition and its related operations and its effectiveness as a variance reduction techniques. The limitations of the pattern recognition system in reducing varlance are discussed in Section 5.4. Finally, Section 5.5 summarizes the chapter.

\section{1) Implementation}

The first requirement of implementation is to make prototypes available to the classifier. In this research prototypes are created after each collision;* however, not until after a particle is lost to

\footnotetext{
*Prototypes could also be created when entering a new geometric region; however, energy and angle variables will be unchanged from the previous prototype.
} 
the system or is tallies can the importance be deternined (this is not true for the point detector, see Section 5.1.3). Figure 5.1 illustrates when the infnzation for the prototypes is extracted from the Monte Carlo probles esd Eigure 5.2 shows when pattern recognition is performed. Inlike t:e Yonte carlo problems used in Chapter IV, for many problens a singie source particle can lead to fultiple contributions to the tally. Such a case is illustrated in Figure 5.3 where the track length in region 2 is talifec. Due to scattering in region 1 , it is possible for a particle to pass through region 2 (and thus be tallied) any nubjer of times before leaving the systen. Because of these Eulti-contributions the pattern classification loop of Figure 5.2 can be entered tany ti=es during the trace of a single source particle.

The pattera ciessizication block can be considered to be a FORTRAR subroutine. Sections 5.1.1, 5.1.2, and 5.1.3 discuss the structure of this subroutine for a sirgle tally, : pultiple tallies, and a point detector tally, respectively, using the information gained in Chapter IV.

\subsection{1) Single Tally}

The majority of Yonte Carlo problems which require importance sampling to reduce the variance are characterized by a seldom occurring tally. Thus the majority of prototype importances will be zero. The problem with zero importance prototypes is that they contain nuch less information than a non-zero tally (i.e., all prototypes wh zero importances look the same to the classifier whereas non-zero inportances can 


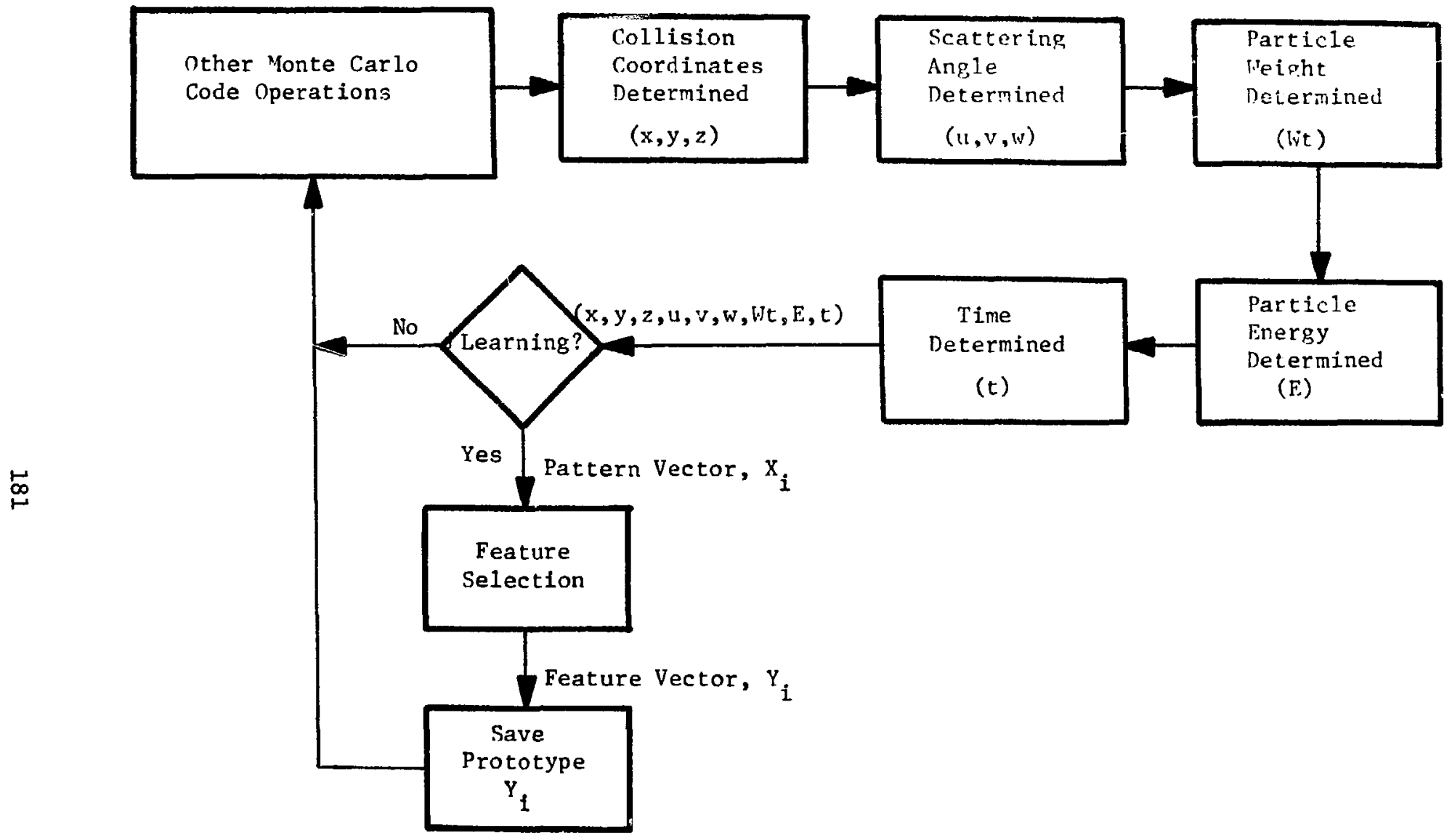

Figure 5.1 Saving Information for prototypes 


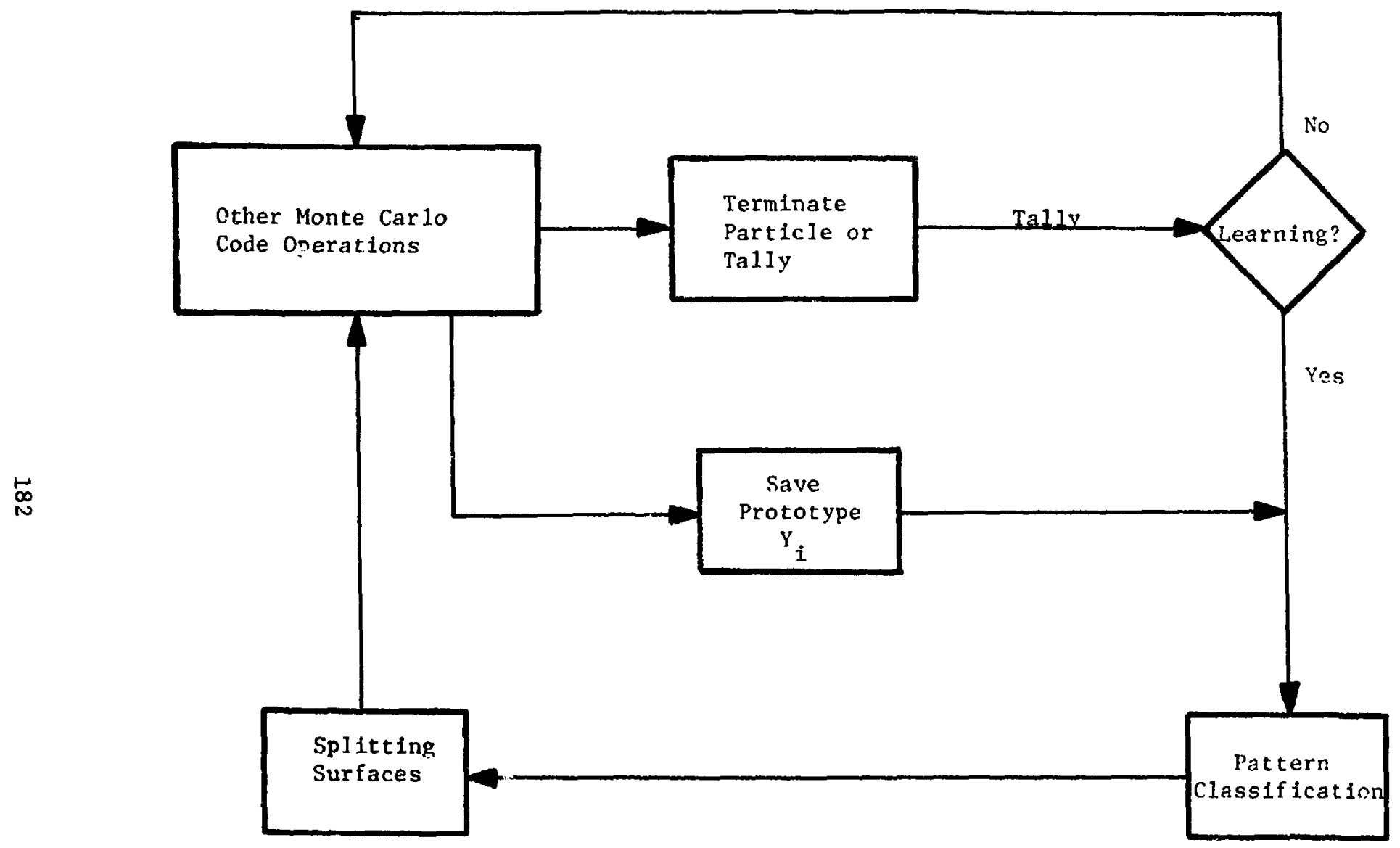

Figure 5.2 Communication between the Pattern Classifier and the Monte Carlo Program 


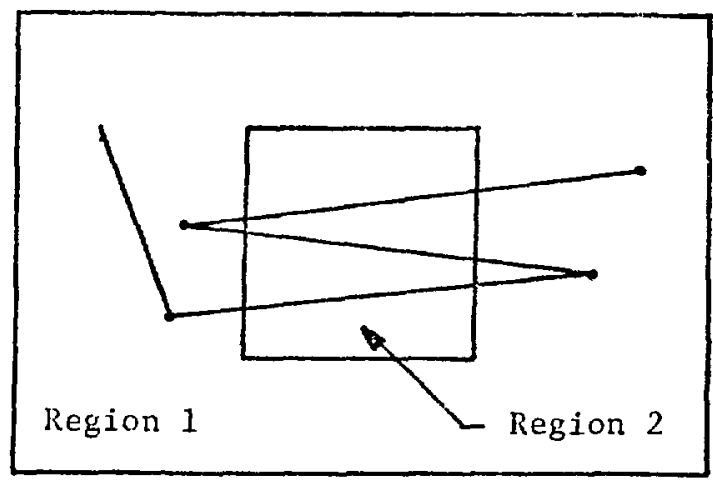

Figure 5.3. Multiple Contributions to a Tally

be classifled among themselves). Therefore the first splitting surface to be learned is the surface separating zero from non-zero importance regions.

The Initial location of this first splitting surface should be well within the class 1 (zero importance) region. Default*values may be used to locate this surface; however, if the user can supply more Information, it should be used in order to speed up convergence. Suitable default values for the components of $\mathrm{v}$ are

$$
\begin{aligned}
& w_{i}=-1.0 \quad i=1, \ldots, N \\
& w_{N+1}=N
\end{aligned}
$$

* A default value is one which is supplied automatically by the computer program; however, the program also allow: for the user to provide a different value. If the user provides a value, it is used; if he does not, the default value is used. 
where it has been assined that the feature selector has nornaliacd a!l feature components to an interval from zero to one (see Section 5.2) such that importance decreases as $y_{i}\left(i=1, \ldots, l_{i}\right)$ increases.

Once this initial location has been set, prototypes can then be used as the Yonte Carlo calculation progresses. This splitting suriace can be utllized wille $\because$ is being learned. By doing this more prototypes are introduced on the class 2 side wich will accelerate the convergence. When the classiaier attains a suitable misclassification rate and variability (as set by the tser or default), weight adjustrent on the first splitting surface can be stopped. During this first fhase, the number of prototypes in eaci class, $H_{i}(i=1,2)$ is calculated. This variable can be used in tne foilowing expression

$$
\left\{\begin{array}{c}
\text { if } F_{2}=\frac{y_{2}}{x_{1}+y_{2}}>R \\
\text { then no zore splitting surfaces are learned }
\end{array}\right\}
$$

to deterune if.core splitting is necessary where $R$ is determined by the user. The first splitting surface (between zero and non-zero importances) divides state space into two regions - in one region particles have a probability $>50 \%$ of contributing to the tally, in the other region the particles have a probability $>50 \%$ of not contributing to the tally. As $F_{2}$ increases less splitting is necessary. 
If $F_{2}<R$ then an additional surface within class 1 is necessary to get more prototypes into the important regions of the problem. However, since all prototypes in class 1 have zero Inportance, a subgoal or sub-tally must be used for classification. One such choice is to observe when a particle enters class 2 according to the student. This is done by checling the sign of $g(Y)$ after each colliston (this is already done since $g(Y)$ is being used to split particles). If it is found that a particle is entering $C_{2}$ (i.e., $g(Y)>0$ ), the particle is tallied (only for the pattern classifier). The prototypes are then sent to the pattern classifier. After the classification process, the particle continues the random walk. However, prototypes are not created until the particle re-enters $c_{1}$ (1.e. the "No" learning branch of Figure 5.1 is used). Tf the particle re-enters $C_{1}$, prototypes are again saved unt1l the particle either enters $C_{2}$ or is lost to the system. Like the $\mathrm{C}_{1}-\mathrm{C}_{2}$ splitting surface, a single source particle can contribute several "sets" of prototypes (one set for each tally). Unlike the $c_{1}-C_{2}$ splitting surface, prototypes are not created after each collision, but only after collisions occurring within $\mathrm{C}_{1}$. With this procedure, class 1 can be subdivided into two sub-classes, class 3 and class 4 where class 4 particles have a 50\% or greater chance of beconing a class 2 particle and class 3 particles have less than $50 \%$. The choice of an intial wor the second splitting surface can be made by using Equation 5.3 


$$
\begin{aligned}
& I_{1}^{w_{1}}=\frac{2\left(I^{w}+1\right)}{\frac{2^{w} N+1}{2^{w}+\frac{1^{w} N+1}{1^{w}}}} \quad I=1, \ldots, N \\
& I_{N+1}=\frac{1}{2}\left(w_{N+1}+2 w_{N+1}\right) \\
& \text { where } I_{1}=\text { Inftial welght of new discriminant function } \\
& 1_{1} w_{1} \text { initial weight of previous discriminant function } \\
& 2 w_{1} \text { final weight of previous discriminant function }
\end{aligned}
$$

which results in a splitting surface located mid-way between the first and last positions of the class 1-2 decision surface. When the second splitting surface has been identified a check is again made on $F_{2}$. If $F_{2}<R$, three things can be done: (1) class 3 can be subdivided (2) class 4 can be subdivided (3) class 3 and 4 can be subdivided at the same time. If $\mathrm{F}_{2}<<\mathrm{R}$, then the third choice is needed since a considerable amount of splitting is necessary to make $F_{2}$ larger. Otherwise the choice between 1 and 2 is decided as shown by relation 5.4 with $\mathrm{I}=3, \mathrm{~J}=4$.

$$
\begin{aligned}
& \text { If } \mathrm{M}_{I}>\mathrm{M}_{\mathrm{J}} \text {, subdivide class } \mathrm{I} \text {. } \\
& \text { If } \mathrm{M}_{\mathrm{J}}>\mathrm{M}_{\mathrm{I}} \text {, subdivide class } \mathrm{J} \text {. }
\end{aligned}
$$

This process continues until efther a maximum number of surfaces have been identifled or $F_{2}>R$. A flow diagram of the above process is shown in Figure 5.4. Table 5.1 lists the eight regions formed by the first 4 splitting surfaces. 


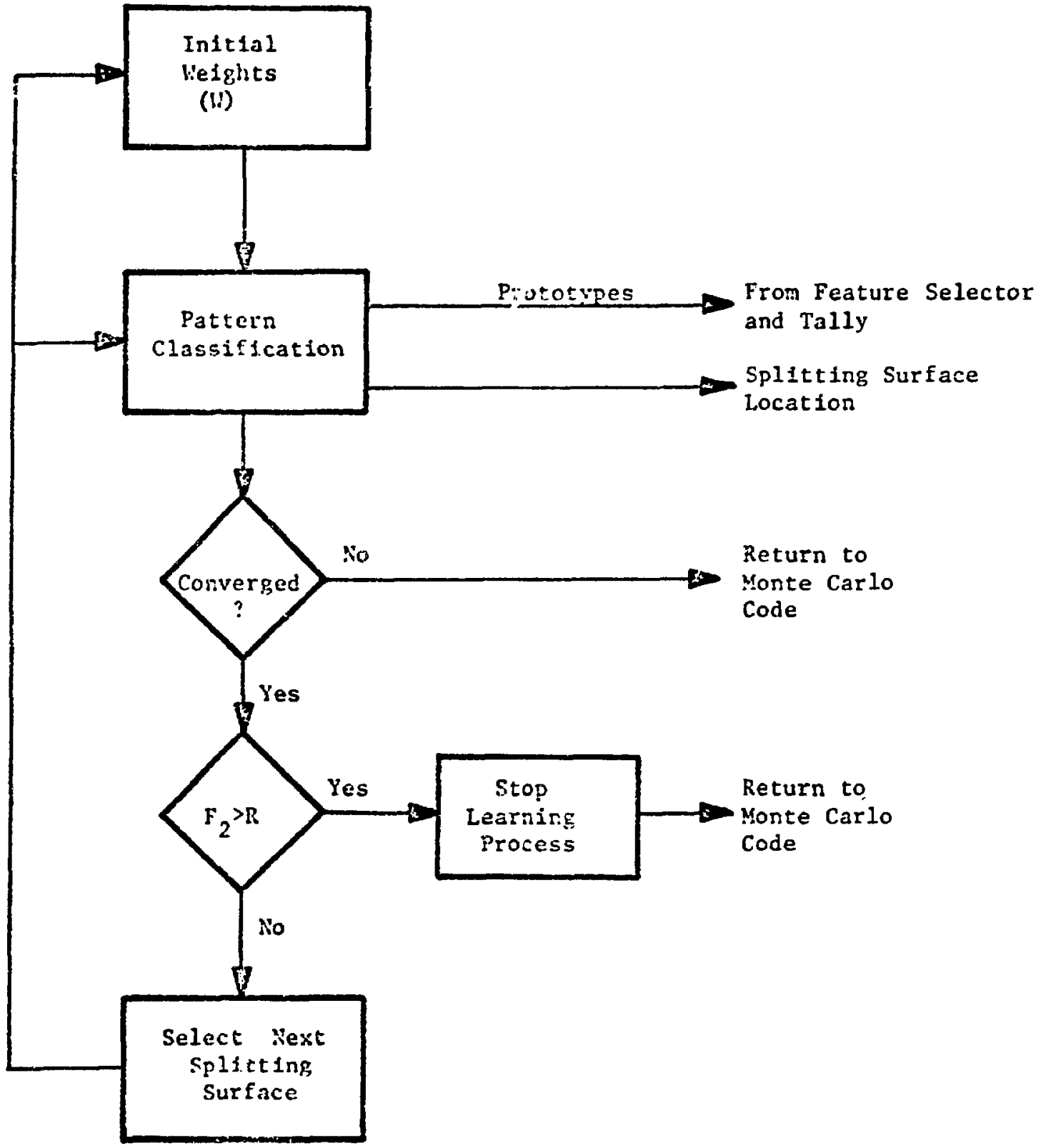

Figure 5.4 Iterative Procedure for Generating Splitting Surfaces 
Table 5.1

$\begin{array}{cl}\text { Class } & \text { Probability of Taljy } \\ 1 & \leqslant 50 \% \\ 2 & \geqslant 50 \% \\ 3 & \leqslant 25 \% \\ 4 & \geqslant 25 \%, \leqslant 50 \% \\ 5 & \leqslant 12.5 \% \\ 6 & \geqslant 12.5 \%, \leqslant 25 \% \\ 7 & \geqslant 25 \%, \leqslant 37.5 \% \\ 8 & \geqslant 37.5 \%, \leqslant 50 \%\end{array}$

\subsection{2) Multiple Tallies}

In the previous section only the splitting surface between classes 1 and 2 depended upon the importances, I, of the prototypes (this is not true if class 2 is subdivided). The problem of multiple tallies consists of defining what is meant by I when more than one taily is considered. This section considers only the $\mathrm{C}_{1}-\mathrm{C}_{2}$ splitting surface. Subdivision of class $C_{2}$ is discussed in Section 4.1 .2 and stibdivision of class $\mathrm{C}_{1}$ is identical to that described in section 5.1.1.

The importance $I_{i}\left(i=1, \ldots, n_{i} n=n o\right.$. of tallies $)$ is the importance relative to a particular tally. Therefore each prototype actually has $n$ importances for $n$ tallies. One could assign a $g(Y)$ for each tally; however, this would consume $\mathbf{n}$ times as much computer time. An easier solution is to assign a probability, $p_{i}$, to each tally such that 


$$
\begin{aligned}
& I=\sum_{1=1}^{n} \delta_{1} I_{1} \\
& \text { where } \delta_{1}=I \text { with probability } P_{1} \\
& \delta_{1}=0 \text { with probability }\left(I-p_{1}\right) \\
& I_{1}=\text { importance with respect to } I^{\prime} \text { th taliy } \\
& I=\text { overall importance of prototype. }
\end{aligned}
$$

This results in a tally with a high $p_{1}$ having wore particles directed towards it. Although the user can aasign the $p_{1}$, it is possible to have this done automatically by using the variance of each tally during the Nonte Carlo calculation. The $p_{1}$ of tallies with high variances should be increased, with low variances decreased. This splits particles so as to produce a uniform variance for all tallies. Although this process affects only the $c_{1}-c_{2}$ surface directly, since all other subclasses of $c_{1}$ are related to this surface, they are also affected indirectly.

\subsection{3) The Point Detector Tally}

The point detector tally described here assumes the same structure as is used in the $\mathrm{MCN}$ code at Los Alamos Sclentific Laboratory ${ }^{19}$, usually used for the tallying of flux at a point. This tally 
differs from other tallies in that after each collision, the contribution to the tally, $x_{i, j}$, given by

$$
\begin{aligned}
& x_{i, j}=w t_{i, j} p_{j} \\
& \text { where } x_{i, j}=\text { contribution to the tally of the } i^{\prime} \text { th } \\
& \text { particle after the } j^{\prime} \text { th collision } \\
& \left(x_{1}=\sum_{j=1}^{J} x_{1, j} \text { where } J=\right.\text { no. of collisions) } \\
& w t_{i, j}=\text { weight of the } i^{\prime} \text { th particle before the } \\
& j^{\prime} \text { th collision } \\
& p_{j}=\text { (probability of scatter) } \times \text { (the probability } \\
& \text { of scattering toward and being detected } \\
& \text { by the point detector at the f'th collision) }
\end{aligned}
$$

is calculated. Unlike other tallies each prototype has a non-zero importance which eliminates the $\mathrm{C}_{1}-\mathrm{C}_{2}$ surface as described in previous sections, Instead an appropriate $\bar{I}$ must be selected which falls within the distribution of $I_{j}{ }^{\prime} s .\left(I_{j}=\right.$ importance of the $j^{\gamma}$ th prototype, not to be confused with $I_{i}$ of Equation 5.5 which is the importance of a prototype wich respect to the $i^{\prime}$ th tally). The median is a good choice for $\bar{I}$ since it is a measure of the number of prototypes. The mean is a less useful measure since it can be strongly influenced by the value of $I_{j}$ (i.e. a few very large $I_{j}$ affects the mean $\bar{I}$ more than a much larger number of prototypes with small $I_{j}$ ). For some cases (especially when the distrtbution of $I_{j}$ is over many orders of magnitude) the mean falls at the tall 
of the distribution which would not be very useful for splitting. Another possible value for $\bar{I}$ is the logarithmic mean (see Equation 5.7)

$$
\bar{I}=\text { logarithmic mean }=\frac{\sum_{j=1}^{N} \log _{10} I}{N}
$$

where $N=$ number of prototypes

$$
I_{j}=\text { Importance of the } j^{\prime} \text { th prototype }
$$

which compensates for distributions that cover many orders of magnitude. An important consideration in the choice of an $\bar{I}$ is the computer time required. Thus, although the median is the best cholce from a statistical point of view (it creates classes which originally have the same number of prototypes in each class) it is also expensive calculationa1ly.

The initial stages of the Monte Carlo calculation are used to calculate $\bar{I}$. During this initial stage weight adjustment is allowed with the intial $W$ chosen the same as described in Section 5.1.1. The splitting surface is not used until a final $\bar{I}$ has been determined. This prevents splitting from influencing the selection of $\bar{I}$. Wher the first splitting surface has converged, $\mathrm{F}_{2}$ is checked (see Equation 5.2). If $\mathrm{F}_{2}<\mathrm{R}$ then the procedure continues as illustrated by Figure 5.4 . As described in Section 5.1 this process continues until either $F_{2}>R$ or a maximum number of surfaces has been created. 
The Importance of a prototype for multiple puint detectors is determined by

$$
\begin{aligned}
& I=\sum_{i=1}^{n} m_{1} I_{i} \\
& m_{i}=\text { weight of tie } i^{\prime} \text { th detector } \\
& I_{1}=\text { importance relative to the } i^{\prime} \text { th detector (same as } \\
& \quad \text { Equation } 5.5 \text { ) } \\
& \mathbf{n}=\text { number of detectors }
\end{aligned}
$$

The weights, $m_{i}$, serve tho purposes. First of all they are used to norralize tallies. This is required when tallies with different units are used (for exasple fissions at a point, captures at a point, flux at a point) and must be noralized to $0<I_{i}<1$. The second purpose of $\mathbb{m}_{i}$ is to direct particles towards certain tallies. This is done by increasing $\square_{i}$ for tallies to which more particles are needed in the same wanner as was suggestei for the $p_{i}$ (see Section 5.1.2). Also like $p_{i}$, optimum $\mathbb{w}_{i}$ can be Iearaed during the calculations or can be supplied by the user.

\section{2) Feature Selecticr:}

As stated in Section 3.4, feature selection is best served for this research if it remains a human function. This can easily be done if the feature selector consists of a user supplied subroutine in much the same was as for source subroutines ${ }^{19}$. The function of the feature selection subroutine is shown in Figure 5.5. The user must supply the following information: 


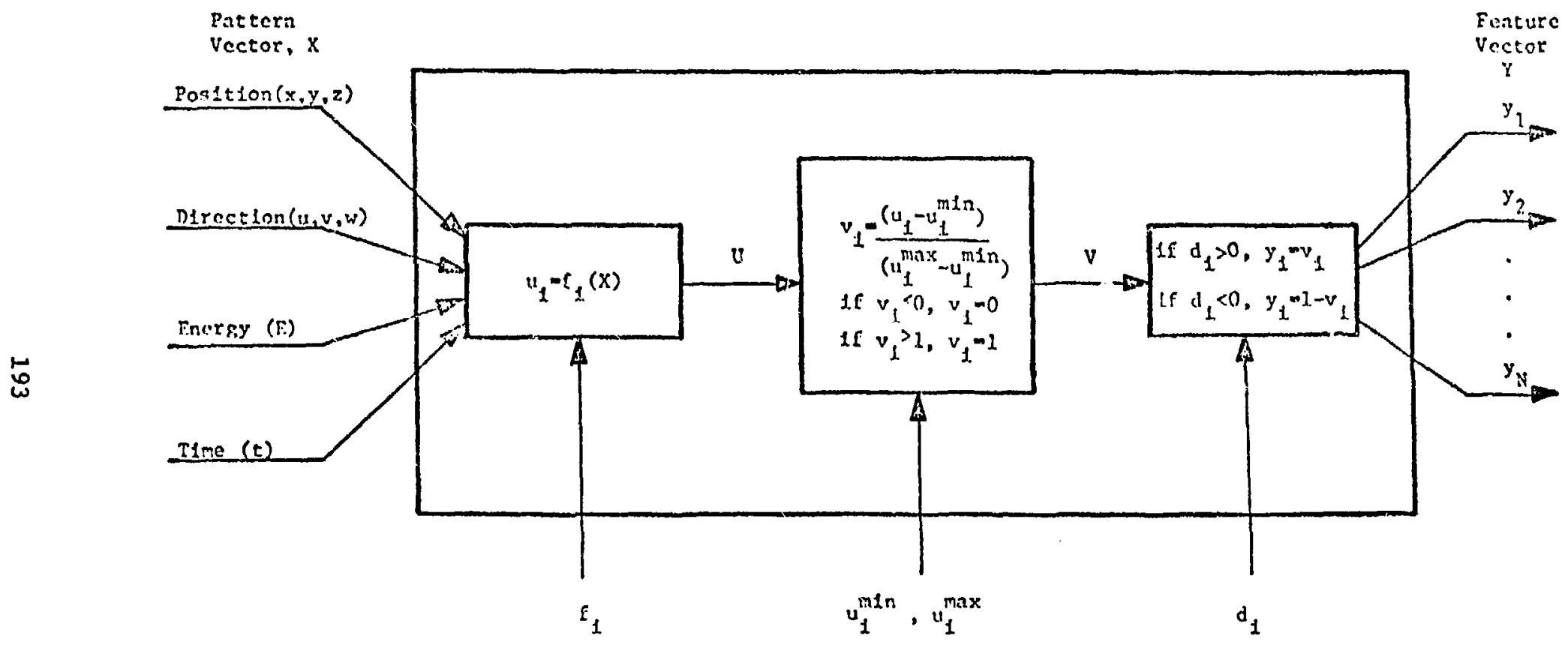

Supplied by the User

Figure 5.5 Structure of the lieature Selector 


$$
\begin{aligned}
f_{i}= & \text { the functional form of the } I^{\prime} \text { th components of feature } \\
& \text { space (e.g., } f_{i}=r=x^{2}+y^{2}+z^{2} \text { ) } \\
u_{i} \text { min }= & \text { the minimum value encountered for the } i^{\prime} \text { th component } \\
& \text { of feature space } \\
u_{i} \text { max }= & \text { the maximum value encountered for the } I^{\prime} \text { th component } \\
& \text { of feature space } \\
d_{1} & +1, \text { if the importance increases as } v_{i} \text { (see Figure 5.5) } \\
& i n c r e a s e s \\
= & -1, \text { if the importance decreases as } v_{i} \text { increases }
\end{aligned}
$$

The selection of the $F_{i}$ can do more to improve the behavior of the pattern recognition system than any other user input. As users gain experience they will undoubtedly gain skill in the selection of $f_{1}$. The following heuristicst or rules of thumb should prove of value in this process.

(1) Omit any variables which are not used in the problem.

(2) Make use of any symmetries in the problem.

(3) If a variable spans several orders of magnitude, use the logarithm of the variable.

(4) Limit the span of a pattern vector component to that range

* Hewristics are non-analytically derived rules usually gained through insight or experience and are used when analytical techniques are inadequate or non-existent. 
over which splitting surfaces are likely to occur. This is done by specifying the range $\left(u_{1}^{\min }, u_{1}^{\max }\right.$ ) to be smaller than the actual range of variable $u_{1}$.

(5) If the itportance varies very weakly with a variable, omit the variable.

(6) Determine what rariables are important from the "tally point of view".

(7) Select features for which the importances either zonotonically increases or cecreases.

Eeuristics 1, 2, and 5 are ai-ed prinarily at reducing the dirensionality of feature space. Eeuristics 3 and 4 are used to prevent surfaces frol being too close to each other in feature space. The results of these heuristics is to experc the space in the vicinity of splitting surfaces. The purpose of heuristic 6 is to select optiou feature components for splitting. Feuristic 7 is aimed at preventing class distributions which cannot be treated with linear discrininant functions. Suggestions for each pattern vector component are given below using the above heuristics as guidelines.

T1me: A great number of transport problems are concernec only with steady state situations. For these problens time should be ocitted Erod all $f_{1}$ (heuristic 1). Problems which do involve time often involve several decades. In this case heurlstics 3 and 4 should be used. Tine will usually no: be conbined with any other variabies to form a new $y_{1}$ but will be used alone. 
Energy: Most transport problems of interest are usually strongly dependent on energy due to the energy dependence of nuclear cross sections. An example of this is the fission cross section which varies from a few barns in the Hev region to several hundred barns in the ev region. Therefore, the probability of a fission occurring varies by two orders of magnitude due to energy alone. Heuristics 3 and 4 are almost always necessary unless dealing with only a very small energy interva1. There are problems in which, over the energy range of interest, the cross sections of the materials involved (for example, carbon) are relatively flat. In such cases heuristic 5 should be executed. Iike time, the energy variable will seldom be combined with other variables.

Spatial Coordinates $x, y, z$ : Heuristic 2 is intended primarily for the spatial coordinates. For example, jf the geometry of a problem Is symmetric about an axis, cylindrical coordinates should be used for the features. Once the geometry has been transformed to cylindrical coordinates, it may be found that the importance does not vary with y or $\theta$ (see Figure 5.6). Then by using $f_{i}=r=x^{2}+z^{2}$, three components $(x, y, z)$ have been reduced to one. An analogous stutuation exists for spherically symmetric problems. Figure 5.7 illustrates a case where heuristic 7 is necessary. By transforming $x$ as given by

$$
f_{i}=\left|\left(x-x_{1}\right)\right|
$$




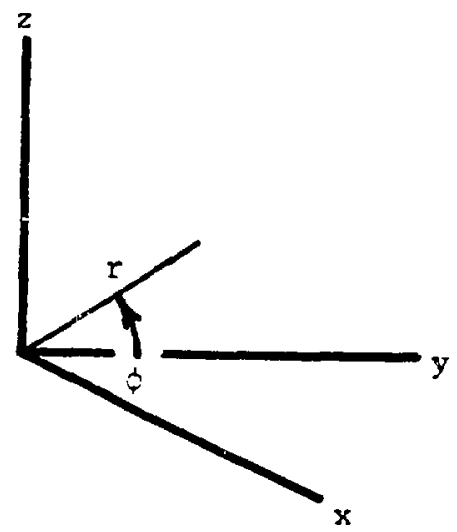

Figure 5.6 Cylindrical Symmetry

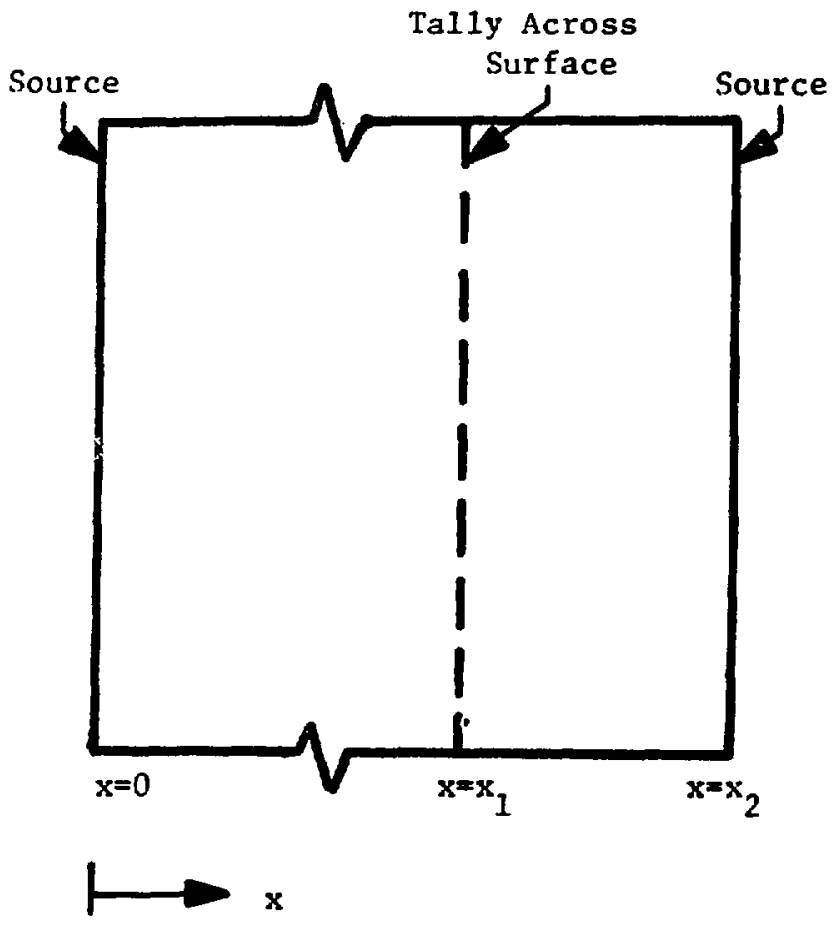

Figure 5.7 Feature Selection to Avoid a Quadratic $g(Y)$

197 
a linear discriminate function can be used. In general the distance to a tally is the best choice of a spatial coordinate (heuristic 6) as given by

$$
f_{i}=d=\sqrt{\left(x-x_{d}\right)^{2}+\left(y-y_{d}\right)^{2}+\left(z-z_{d}\right)^{2}}
$$

where $x_{d}, y_{d}, z_{d}$ are the coordinates of the tally

Such a choice also reduces the number of features. Spatial features will usually not require the use of heuristics 3 and 4 .

Direction Coordinates $(u, v, w)$ : The direction cosines are sinilar to the spatial coordinates in that they are usually better combined. For example, the cosine of the angle between the present line of flight (vector $=v_{1}$ ) and the direction to the tally (vector $=v_{2}$ ) given by

$$
\mathrm{F}_{i}=\cos \phi=\frac{v_{1} \cdot v_{2}}{\left|v_{1} v_{2}\right|}
$$

Is similar to a (Equation 5.10). Using the above feature also allows lincar discrininant functions (heuristic 7 ). As in the case of the spatial coordinates, cylfudrical and spherical symotry can of ten reduce the number of angular features necessary. 
The problem illustrated in Figure 5.8 will be used to demonstrate the proper selestion of features. In this problem material 2 is highly absorbing and material 1 is only slightly absorbing but has a moderately

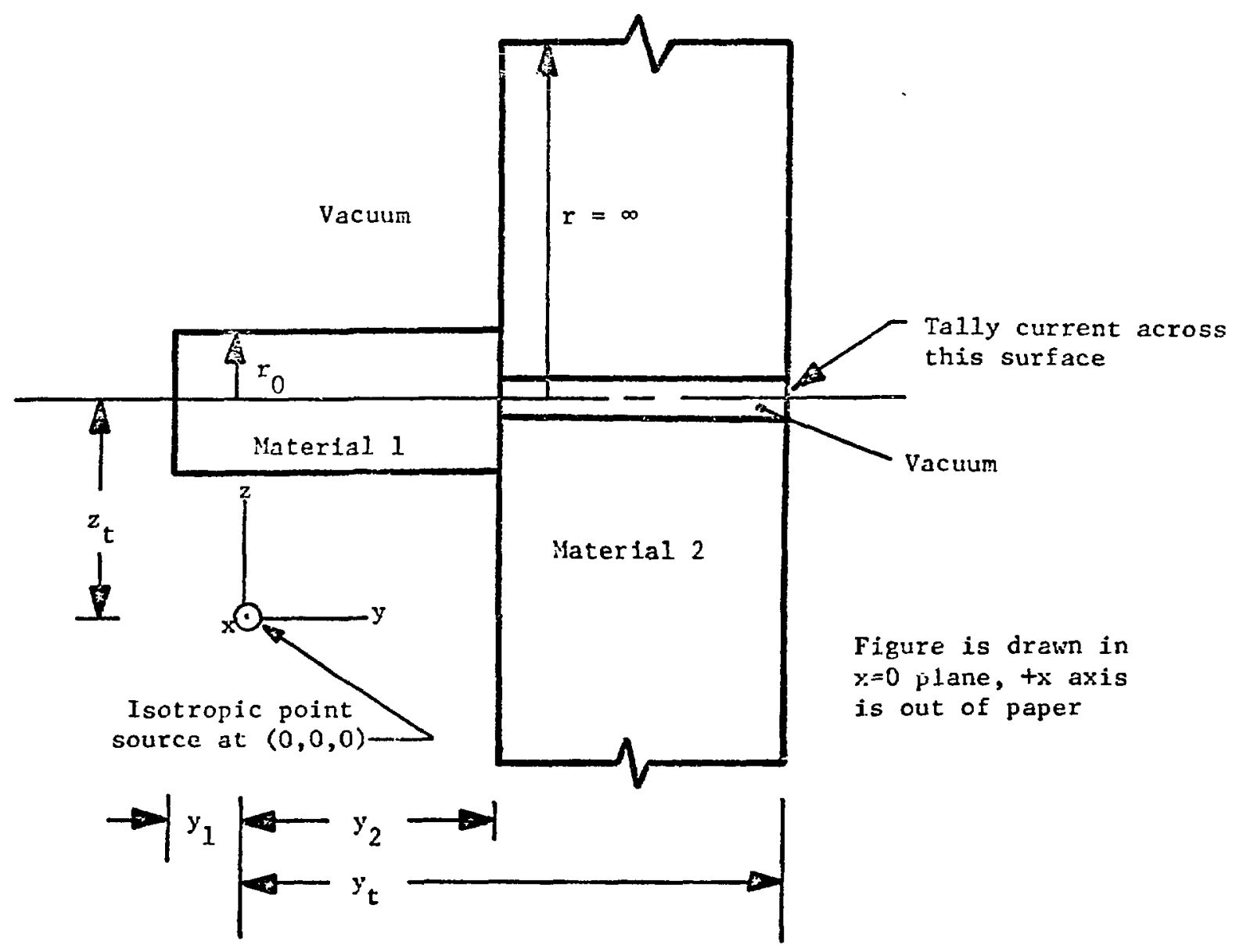

Figure 5.8 Sample Problem for Feature Selection

high scattering cross section. An isotropic source of neutrons is 10cated at the origin. It is assumed that all cross sections are flat with energy and that time is of no interest. The tally consists of counting the number of neutrons which cross the surface located at 
$y=y_{t} \quad$ The material reglons shown are cylindrically symmetric about the line drawn through the point $\left(0,0, z_{t}\right)$ and parallel to the $y$ axis.

The following $f_{1}$ would be suitable for the feature vector of this problem:

$$
\begin{aligned}
& f_{1}=y \\
& f_{2}=r=\sqrt{\left(z_{t}-z\right)^{2}+x^{2}} \\
& \mathrm{f}_{3}=\mathrm{v} \cdot \mathrm{v}_{\mathrm{d}}\left|\mathrm{v} \mathrm{v}_{\mathrm{d}}\right| \\
& u_{1}^{\text {min }}=-y_{1} \quad u_{1}^{\max }=y_{t} \\
& u_{2}^{\min }=0 \quad u_{2}^{\max }=r_{0} \\
& u_{3}^{\min }=-1.0 \quad u_{3}^{\max }=1.0 \\
& \text { where } \mathrm{v}=\text { direction cosine with } \mathrm{y} \text { axis } \\
& v_{d}=\text { direction cosine to point }\left(0, y_{t}, z_{t}\right)
\end{aligned}
$$

The previous seven heuristics relate to the above selection as follows:

Heuristic 1. Since cross sections are flat, the problem is independent of energy and as was stated time is of no interest. Therefore time and energy do not appear as variables in any of the $f_{i}$

heuristic 2. The form of $\mathrm{E}_{2}$ was chosen due to the symmetry of the problem. This reduced the number of variables and is also a more effective variable for splitting.

lleuristic 3. Not used. 
Heuristic 4. Tinis heuristic was used to set $u_{2}$ max. For $r>r_{0}$ particles all belong to the same class thus limiting splitting to $r<r_{0}$. The other $u_{i}$ aax and $u_{i}$ min are set to the linits of the problen.

Eeuristic 5. $\quad$ iot used.

Feuristic 6. This heuristic influenced the choice of all three $f_{i}$. If one is at the tally looking at contributing neutrons, almost all the neutrons will be coning down the channel sxom Eaterial 1 (ie come from naterial 2 since it is highly absorbing). Because of this the probability of a neutron arriving at the tally cepends prinariiy on the $f_{i}$ chosen.

Feuristic 7. \&s $\hat{f}_{1}$ increases from $-y_{1}$ to $s_{t}$, the probability that it is tallied also increases monotonically. This is also true for $f_{2}$ and $E_{3}$.

Although the selection of features does reguire user information, this information is qualitative in rature as opposed to the quantitative information required by normal geometry splitting.

\section{3) Tining Considerafions and Fffectiveness}

The purpose of this section 1s to determine the factors necessacy for estimating the effectiveness of state space splittirg using pattern recognition. The only definitive way to deteraine the effectiveness as given by Equation 2.16 is to apply the technique to a full scale 
Yonte Carlo Code and use it both with and whout the variance reductica for a large nutier of probleas. The approach taken in tias section is to compare the various operations involved In state space splitting as opposed to conventional splitting (Section 5.3.1). The additional operations involved in using pattern recognition to icentif: the splitting surfaces are ther described (Section 5.3.2). Aitscugh this analysis does not result in an absolute evaluation for 212 probleas, some assuptions can be wade concerning the effectiveress as coapared to presently used splitting technique.

\subsection{1) State Space Splitting vs. Conventional Splitting}

The basis of this conparison is the current version of the MCX neutron Monte Carlo code at Los Alamos Scientific Laboratory tains both energy splitting and geowetry splitting. For the eaergy splitting the user specifies a nu-ber of energy splitting surjaces, $E_{i}$, $i=i, \ldots, N$ ( $N=$ number of splitting surfaces) and the ratio for spititing between two energy regions (separated by $E_{i}$ ) $R_{i}, i=1, \ldots, x$. Fìe energ: of the particle is checked after each collision. If the eneray irops below an $E_{i}$, the particle is split into $R_{i}$ particles with weights equal to the particles originel weight divided by $R_{i}$.

Each geometric cell or region (see Appendix $J$ ) is assigned an importance $I_{i}$. Whenever a particle crosses a surface $S_{j}$ wich bounds two or nore cells, a check is made to determine which cell the particle is entering. This check is made by: (1) determining which cell or cells are on the other side of $s_{j}$ (2) If more than one cell is on the 
other side of $S_{j}$ the senses (see Appendix $J$ ) are calculated with respect to the bounding surfaces (with the exception of $s_{j}$ ) of each cell (3) the cell for which all the senses agree is used as the next cell (1f there is only one cell, it is used). The importance of the new cell, $I_{n}$, is then compared with the importance of the previous cell, $I_{0}$, and the operations indicated in Figure 5.9 ars performed. In the case af splitting, if the ratio of importances $I_{n} / I_{0}$ is a non-integer value given by

$$
R=I_{n} / I_{0}-J
$$

where $J=$ largest integer that will go into $I_{n} / I_{0}$,

then $J+1$ particles are created with probability $R$ and $J$ particles are created with probability $(1-R)$. Then particles are split one of the particles is continued and the others are banked* (i.e., the state space description of each is saved) until the first particle leaves the system, at which time the banked particles are continued. Of the above process, the only time used for splitting and Russian roulette is the time spent in the operations shown in Figure 5.9 plus the time spent in banking particles. The average time per particle spent purforming these operations will be designated $\Delta t_{1}$, Unfortunately, this is not the only

\footnotetext{
* The banking of a particle consists of recording its state space description $(x, y, z, u, v, w, E, t)$ and its weight, wt, on a pushdown list. This list is so constructed and maintained that the next particle retrieved from the list is the last particle placed on the list.
} 


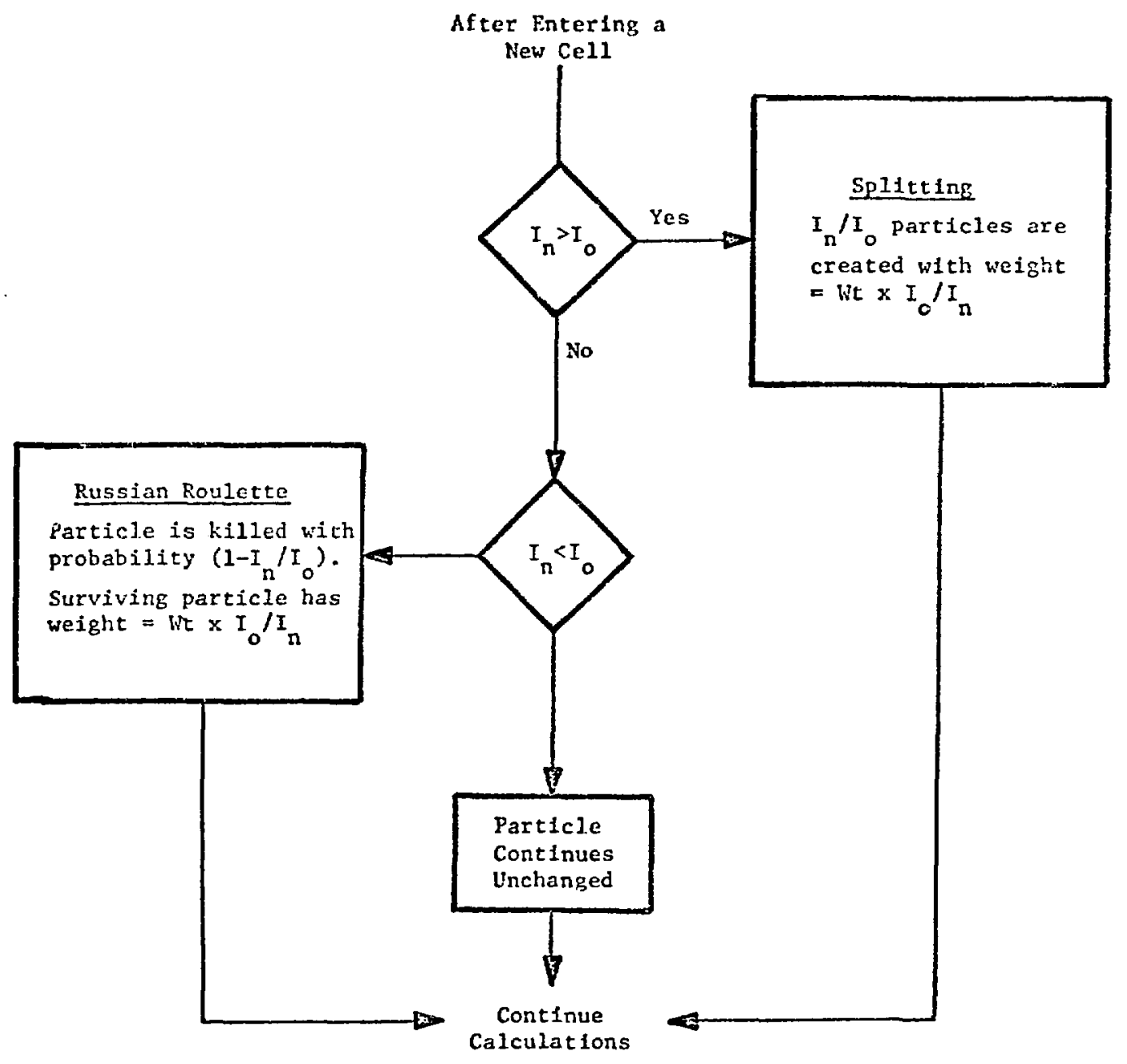

Figure 5.9 Conventional Geometry Splitting 
increase in time due to the splitting process. Because splitting is often desired at surfaces which are not matertal boundarles, the user must subdivide the already existing cells into smaller cells in order to split at the surfaces he desires. The introduction of these new "splitting cells and surfaces" means that more intersections will have to be calculated and more senses will have to be checked. It also requires more calculations of the distance until a colísion. These three operations can consume a considerable amount of time (the najority of computer time spent is often spent in these geometry related calculations). In fact, for sone problems, the additional time required by these operations can completely offset any gains due to variance reduction. The average time per particle spent due to increased geometry calculations caused by "splitting cells" will be denoted as $\Delta t_{2}$. If the additional time per particle spent due to energy splitting is designated $\Delta t_{3}$, then Equation 5.13

$$
\begin{aligned}
\Delta t_{g}=\Delta t_{1}+ & \Delta t_{2}+\Delta t_{3} \\
\text { where } \Delta t_{1}= & \text { time to perform processes of Figure } 5.9 \\
& \text { plus balking of particles } \\
\Delta t_{2}= & \text { time required to perform geometry calculations } \\
& \text { due to additional "splitting cells" } \\
\Delta t_{3}= & \text { time required for energy splitting }
\end{aligned}
$$

gives the total additional time spent due to the use of splitting and Russian roulette. 


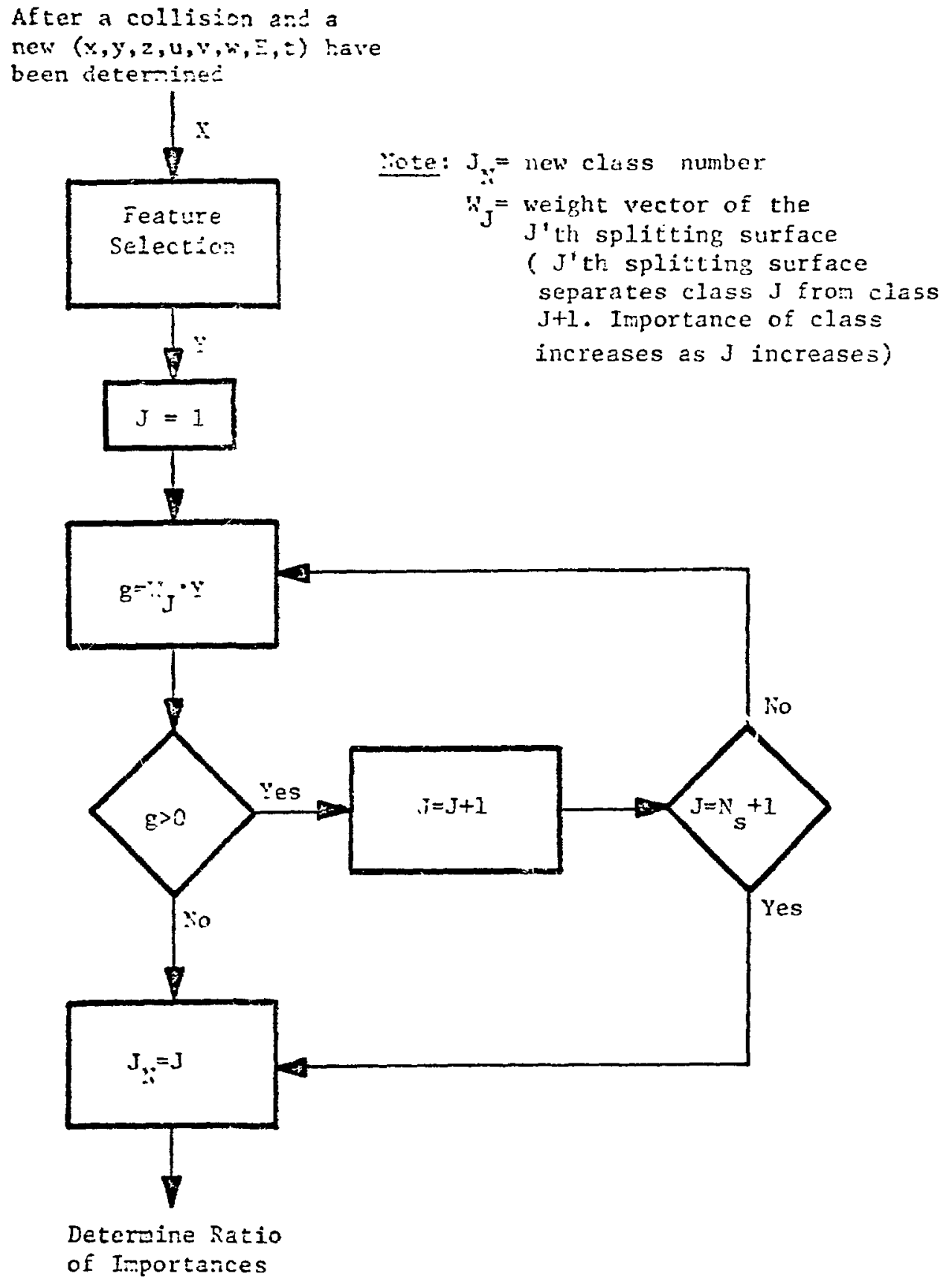

Figure 5.10 Determining the New Class for State Space Splitting 
If state space splitting is used with known splitting surfaces (i.e., $\mathrm{H}$ is known) the operations shown in Figure 5.10 are performed after each collision. Once the new class, $J_{n}$, has been found it is used with the old class, $J_{0}$, to determine the ratio of Importances given by

$$
I_{n} / I_{0}=2^{\Delta J}, \quad \Delta J=J_{n}-J_{0}
$$

After this ratio has been calculated, the procedure illustrated in Figure 5.9 is used. Therefore, using state space splitting, the total additional time per particle, $\Delta t_{s}$, is given by

$$
\begin{aligned}
\Delta t_{s}=\Delta t_{4}+ & \Delta t_{1} \\
\text { where } \Delta t_{4}= & \text { average time spent per particle to perform } \\
& \text { operations of Figure } 5.10 \\
\Delta t_{1}= & \text { see Equation } 5.12 .
\end{aligned}
$$

Subtracting Equation 5.15 from 5.13 results in

$$
\Delta t_{g}-\Delta t_{s}=\left(\Delta t_{2}+\Delta t_{3}\right)-\Delta t_{4}
$$

All three of these parameters $\left(\Delta t_{2}, \Delta t_{3}\right.$, and $\left.\Delta t_{4}\right)$ are dependent upon the amount of splitting used. The parameter $\Delta t_{2}$ is also very dependent on the number of "splitting cells" and the complexity of the surfaces involved (higher order surfaces require more time to calculate intersections). The parameter $\Delta t_{4}$ is dependent on the complexity and number 
of the $f_{1}(Y)$. A great deal of experitentation with fany different Yonte Carlo problens would have to be run to decermine bibctice it or $\Delta t_{s}$ is larger. It is most probajle that each technique has a class of probleas for which tt operates ciuclier.

If both teahninues are usat to represent the sase surfacos, the relative effectivencss can be calculated by comparing the aboue ti=es only. However, this is not the case since the comentionsl teshniọe splits only in energy and postion space (incepencichely) and tie other approach splits in state space. Furthertore, the spitetsti surfaces of the conventional approach arc the zesults of hetristic guesses by the user; whereas, in the stace space approach, the surfaces are the result of the pattern recogaizer. Figtre 5.11 co-osscrases the advantage of splitting in stace space for the two dicensional problen considered in Section 4.4. The solld line indicates tie splittir.g surface found by pattern recognition. The dashed lines are a fin of the splittina surfaces possible if geonetry splitting is usec alcne. As can be seen there is a great arount of deviation regardiess of wich geowetry splitting surface is chosen. The problen is caused by the fact that one cannot split along constant contours of I usirg geocetry and energy splitting only. As a result, many particles are split which should not be. As a result, $\mathrm{H}_{\mathrm{e}}$ (see Equation 2.15) will in general be nuch smaller for state space splitting than for the conventional splitting approach. 


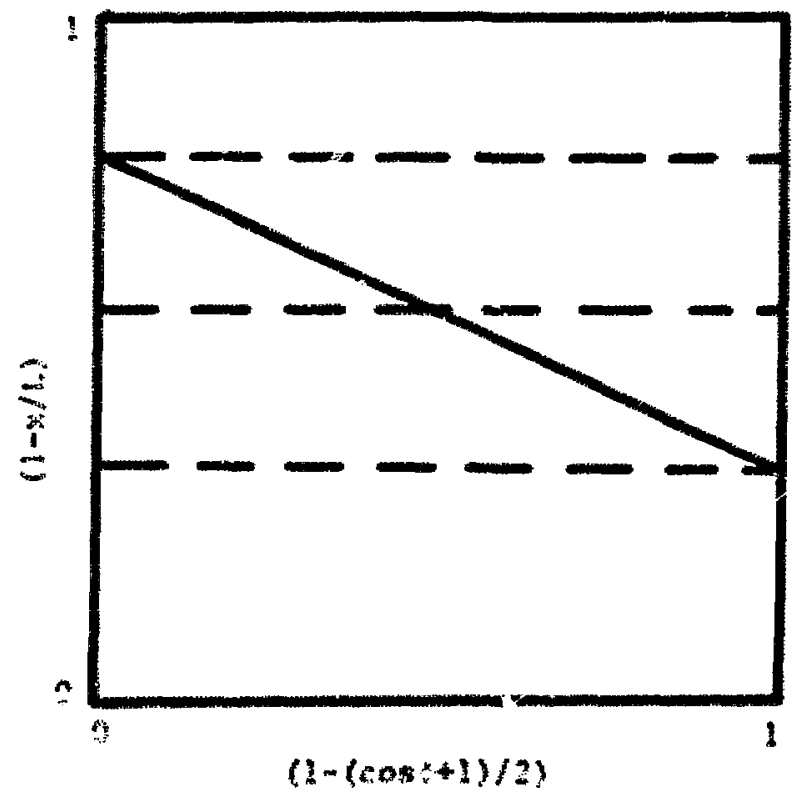

Fisto Probleta of seccion 4.2

\subsection{2) time Spcint Cor Baceern Recognicion}

Section 5.3.1 discussed the time required to split particles in state space. This section discusses the additlonal time required to learn the splitting surfaces and includes:

(1) $T_{f}=$ the additional time requited to save prototypes (see Flgure 5.1)

(2) $T_{P}=$ the tiwe required to classify patterns and adjust $W$ (see Sections S.2.3, 4.4.3, and 4.5) 

(3) $T_{\mathrm{m}}=$ time required to "manage" the splitting surface selection (see Figure 5.4).

The above operations are different from those described in Section 5.3.1 since they are only performed while the splitting surfaces are being learned. Because of this, the time spent for pattern recognition is distributed only over those particles which extst while the pattern recognizer is being used.

The discussion in this section assumes that a point detector tally is not used and that the splitting surfaces are learned one at a time (i.e., one surface has passed the convergence test before another is begun) as described in Section 5.1.1. The following notation is used throughout this section:

$$
\begin{aligned}
S_{k, j}= & \text { the splitting surface between classes } k \text { and } f \text { where } \\
& \text { the } k \text { and } f \text { are defined as given by Table } 5.1 \text {. The } \\
& I^{\prime} \text { th splitting surface refers to surface } S_{21-1,21^{\circ}} \\
N_{1}= & \text { the average number of collisions per source particle } \\
& \text { In a particular Monte carlo calculation before the } \\
& I^{\prime} \text { th surface is learned but after the }(i-1)^{\prime} \text { th surface } \\
& \text { is learned. ( } N_{1}=N_{1} \text { before any surfaces are learned) } \\
h_{1}= & \text { average fraction of } N_{1} \text { which occur in } C_{i} \text { before the } \\
& \left.I^{\prime} \text { th surface } 1 \text { s learned but after the ( } 1-1\right)^{\prime} \text { th surface } \\
& \text { is learned ( } h_{1}=h_{1} \text { before any surface is learned }=1 \text { ). }
\end{aligned}
$$


$Q_{i}=$ the average number of times a source particle contributes to the tally (or class sub-tally) while the $i^{\prime}$ th surface is being learned.

$B_{I}=$ number of source particles required to learn the $I^{\prime}$ th splitting surface.

$P_{1}=N_{1} h_{i} B_{j}=$ number of prototypes required to learn the $I^{\prime}$ th splitting surface.

$M_{S}=$ total number of splitting surfaces learned. $M_{s}$ is either supplied by the user or is determined by the $F_{I}$ test (see Figure 5.4).

The splitting surfaces are learned in the following order:

$$
s_{1,2}, s_{3,4}, s_{5,6}, s_{7,8}, \ldots \ldots s_{2 M_{s}-1,2 M_{s}}
$$

$\mathrm{T}_{\mathrm{f}} \quad$ (saving prototypes)

The total time spent transforming vectors from pattern to feature space is given by

$$
\begin{gathered}
\begin{array}{c}
T=t_{f} \sum_{i=1}^{M_{s}} N_{i} h_{i} B_{I}=t_{f} \sum_{i=1}^{M} P_{i} \\
\text { where } t_{f}=\text { average time spent transforming one prototype } \\
\text { (see Figure 5.5) }
\end{array}
\end{gathered}
$$


The time parameter $t_{f}$ depends on the number and computational complexIty of the $f_{i}$ (see Figure 5.5) involved and like the paraneters $N_{i}, h_{i}$, and $B_{1}$, Is highly problen dependent. The $v_{1}$ (see Flgire 5.5) are of ten already calculated within the Monte Carlo Code and therefore need only be stored.

After the first learning surface has been identified, the feature vector is calculated after every collision in order to utilize state space splitting (see Section 5.3.1). Since this operation need not be performed twice, the additional time used to transform pattern vectors is given by the equation

$$
T^{\prime}=t_{f} N_{1} B_{1}
$$

If the additional time spent in storing the $\mathrm{Y}_{i}$ is designated by $T_{s}$, then $\mathrm{T}_{f}$ is given by the following equation

$$
T_{f}=T^{\prime}+T_{s}=t_{f} N_{1} B_{1}+T_{s}
$$

In general $\mathrm{T}_{s} \ll \mathrm{t}_{\mathrm{f}} \mathrm{N}_{1} \mathrm{~B}_{1}$ resulting in the equation

$$
T_{f} \approx T^{\prime}=t_{f} N_{1} B_{1}
$$


$T_{p \quad \text { (pattern classifier) }}$

The time spent by the pattern classifier has been discussed in Sections 4.2.3, 4.4.3, and 4.5 for the pattern classifiers used in this research and are summarized in Tables 4.5 and 4.7 . It should be noted that these times indicate a maximum of the values to be expected. Very simple changes in the FORTRAN coding can lead to large reductions in time. Some examples of these changes are:

(1) For non-point detector tallies where the classes are determined by "tally" or "no tally", the importance $I_{i}$ need not be calculated. In such a case all prototypes in a "set" (see Section 5.1.1) belong to the same class. The only test necessary to determine classification is to test the tally for zero, non-zero. For the problems run in Chapter IV, this results in changing Equation 4.9 to Equation 5.21 thus reducing $T$ by $(N-B) t_{A}$.

$T=B t_{A}+N\left(t_{L} f_{L}+t_{C} f_{C} f_{L}\right)$

where $B=$ number of source particles

(2) The dot product of two vectors $(W \cdot Y *$ and $Y * \cdot Y *)$ can be performed much quicker by using system functions (for example DOTPRO ${ }^{39}$ at LASL). The larger the dimensions of $\mathrm{W}$, the more time can be saved. In this research each vector element was multiplied in FORTRAN. 
(3) Vector addition $\left(W_{i+1}=W_{i}+c Y^{*}\right)$ can be performed much faster using systen functions (sach as ADDVEC ${ }^{10}$ at 1.ASI.) In this research cach element was added separately using a ForTan statement for each element.

Since the purpose of this research is not to develop an optimum FORTRAN program, these operations have not been used since the more stralghtforward multiplication and additions are easier for the reader to follow (see Appendices D, F, H, I).

The total time spent by the pattern classifler to learn $\mathrm{N}_{\mathrm{s}}$ splitting surfaces is given by liquation 5.22 .

$$
\begin{aligned}
& T_{p}=T_{1}+T_{2}+\ldots \ldots \ldots T_{M_{s}} \\
& T_{p}=\sum_{i=1}^{M}\left(t_{1} f_{L}+t_{C i} f_{i} f_{L}\right) P_{i}+t_{A} \sum_{i=1}^{s} Q_{i} B_{i}
\end{aligned}
$$

where: $t_{A}, t_{L}, t_{C}$ are given by Equation 4.9

$$
\begin{gathered}
I_{L} \text { and } f_{C} \text { are the same as } f_{L} \text { and } f_{C} \text { of: Equation } 4.9 \\
\text { except that they refer to prototypes used } \\
\text { In learning the } I^{\prime} \text { th splitting surface } \\
T_{i}=\text { time spent learning surface } i
\end{gathered}
$$




\section{T}

The time $I_{m}$ includes the followirg operations (see Figure 5.4):
(1) Intial Weight Selection
(2) Convergence testing including the calculations involved In deteraining the misclassification rate and variability.
(3) Spliting Surface Selection including $F_{2}>R$ test.

Operations (1) and (3) are only performed $M_{s}$ times throughout the entire Monte Carlo calculation and therefore their times will be ignored compared to operation (2) which is orders of magnitude more time consuming. The convergence is tested after every $C_{T}$ source particles. The choice of $C_{T}$ depends upon the number of source particles required for convergence, $B_{i}$. However since $B_{i}=P_{i} /\left(N_{i} h_{i}\right)$ and since $P_{i}$ does not vary greatly from problem to problem $\left(P_{i} \sim 4,000\right.$ from Chapter IV), $B_{i}$ can be approximated from the above relationship ( $h_{i}$ and $N_{1}$ are approximated within the Monte Carlo calculation). As experience is gained with this technique, a suitable $C_{T}$ can probably be user specified.

Calculation of the misclassification rate involves storing the number of prototypes and the number of misclassified prototypes. The former is already performed by the classifier for each class and the latter consists of incrementing a counting register every time a prototype is misclassified. A ratio of the two is then performed after every $C_{T}$ source particles. 
The calculation of the variability is more complex. For every feature vector component, $y_{i}$, there is an intercept of the decision surface with the $y_{i}$ axis which is given by

$$
\begin{gathered}
A_{1, j}=\text { Intercept with } y_{1} \text { axis after } j^{\prime} \text { th prototype }= \\
-\frac{w_{N+1, j}}{w_{1, j}} \quad 1=1, \ldots, N
\end{gathered}
$$

where $\mathrm{N}=$ number of feature vector components

$$
\begin{aligned}
w_{1, j}= & I^{\prime} \text { th weight component of } W_{j} \text {, where } W_{j} \text { is the weight } \\
& \text { vector after the } j^{\prime} \text { th prototype }
\end{aligned}
$$

The variability of each intercept is given by

$$
\sigma_{i}=\frac{\left[\sum_{j=1}^{J}\left(A_{i, j}-\bar{A}_{i}\right)^{2}\right]^{\frac{1}{2}}}{\sqrt{J} \bar{A}_{i}}=\frac{A_{i}^{2}-\frac{2}{A_{i}}}{\bar{A}_{i}}
$$

where $\mathrm{J}=$ number of prototypes

$$
\begin{aligned}
& \bar{A}_{1}=\sum_{j=1}^{J} \frac{A_{1, j}}{J} \\
& \overline{A_{1}^{2}}=\sum_{j=1}^{J} \frac{A_{1, j}^{2}}{J}
\end{aligned}
$$


Thus for cach $y_{i}$ both the first and second moments of $A_{i, j}$ must be accumulated. If the J'th prototype is misclassified the accumulated moments are given by

$$
\begin{aligned}
& \sum_{j=1}^{J} A_{i, j}=\sum_{j=1}^{J-n} A_{i, j}+(n-1)\left(A_{i, J-1}\right)+A_{i, J} \sum_{i=1, \ldots, N} \text { (5.25a) } \\
& \sum_{j=1}^{J} A_{i, j}^{2}=\sum_{j=1}^{J-n} A_{i, j}^{2}+(n-1)\left(A_{i, J-1}^{2}\right)+A_{i, j}^{2} \quad i=1, \ldots, N \text { (5.25b) } \\
& \text { where } n=\text { number of prototypes since the last } \\
& \text { misclassified prototype }
\end{aligned}
$$

If the $J^{\prime}$ th prototype is not misclassified then the accumulated moments are given by

$$
\begin{aligned}
& \sum_{j=1}^{J} A_{i, j}=\sum_{j=1}^{J-n} A_{i, j}+n\left(A_{i, j}\right) \quad i=1, \ldots, N \\
& \sum_{j=1}^{J} A_{i, j}^{2}=\sum_{j=1}^{J-n} A_{i, j}^{2}+n\left(A_{i, j}\right) \\
& i=1, \ldots, N
\end{aligned}
$$


After every $C_{T}$ source particles the varlability of each intercept is found using Equation 5.24 and the accumulated values of Equation 5.25. Each $\sigma_{i}$ can be tested separately or combined to give an overall variability as given by

$$
\sigma=\frac{\prod_{i=1, N}^{N} \sigma_{i}}{\sqrt{\sum_{i=1}^{N} \bar{A}_{i}^{2}}} .
$$

The time required to do these operations is summarized by the relation

$$
T_{m} \approx c_{I} \sum_{i=1}^{M} I_{s} c^{P_{i}}+\frac{t_{v}}{C_{T}} \sum_{i=1}^{M} B_{i}
$$




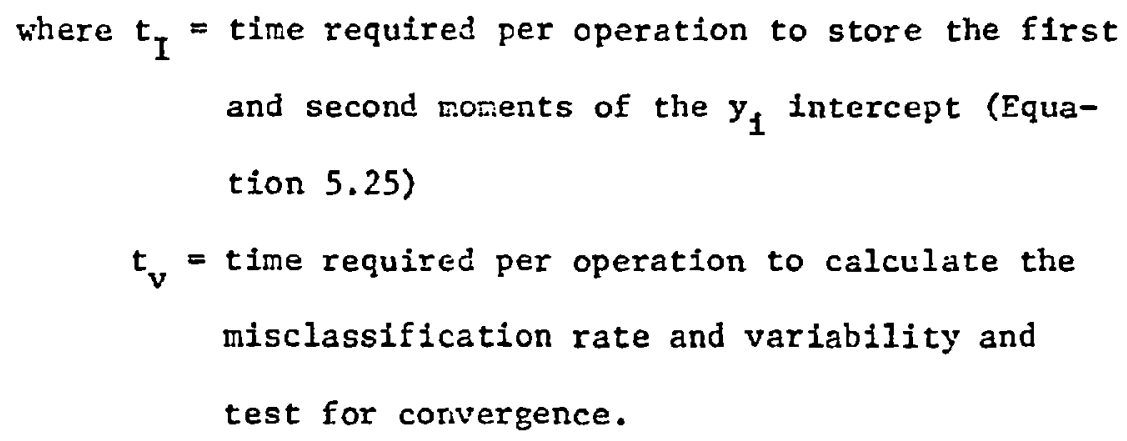

Using a FORTRAN code to simllate these operations results in the following approximate values for $t_{I}$ and $t_{v}$.

$t_{\mathrm{I}} \approx 4.4 \times 10^{-7} \mathrm{iN}$ seconcis

$t_{\mathrm{v}} \approx 6.7 \times 10^{-7} \mathrm{~N}$ seconds

where $N=$ number of features

Combining Equations 5.20, 5.22, and 5.27 results in

$$
\begin{aligned}
& T_{P R}=T_{f}+T_{p}+T_{m} \\
& \approx t_{f} P_{1}+\sum_{i=1}^{M_{S}} P_{i}\left[t_{L I} f_{L}+f_{i} f_{c i}\left(t_{L}+t_{I}\right)+\frac{\left(t_{A} Q_{i}+\overrightarrow{t_{T}}\right)}{N_{I} h_{i}}\right]
\end{aligned}
$$


The above parameters fall into the following groups:

(I) $t_{A}, t_{L}, t_{C}, t_{I}, t_{V}$ are constants which depend only on the programing efflciency used in the pattern classifier

(2) $N_{i}, Q_{i}, h_{i}, i_{i}, M_{s}$ are parameters which depend primarily on the characteristics of the Monte Carlo problem involved

(3) $t_{I}, f_{I}, C_{T}$ are parameters whose values depend on user specification.

The timing parameters from Section 4.5 and Equation 5.28 are summarized in Table 5.2 for the deterministic classifier and $\mathrm{N}$ features. If it is assumed that the 4000 prototypes found necessary for convergence in Chapter IV are characteristic of all splitting surfaces (actually it should be less for each new surface that is learned since the initial guess for $W$ improves with the number of splitting surfaces) and if no buffer zones are used $\left(C_{I} f_{L}=1.0\right)$, Equation 5.29 reduces to $T_{P R} \approx 4 \times 10^{3} t_{f}+4 \times 10^{-3} \sum_{i=1}^{M} 0.3+1.2 f_{i} f+N\left(0.7+2.7_{i} f_{C}\right)+\frac{\left(4.10_{i}+\frac{0.67 N}{C_{T}}\right)}{N_{i} h_{i}}$

If it is further assumed that:

(i) $i^{\bar{x}_{c}}=0.3$ for all $i$ (1.e. a large amount of overlap)

(2) $\mathrm{C}_{\mathrm{T}}=10$ (a very conservative choice considering the value of $P_{t}$ )

(3) $\mathrm{N}=5$ (the maximum using unreduced pattern space is 8 ) 


\section{Table 5.2}

$\begin{array}{ll}\mathrm{t}_{\mathrm{A}} & 4.1 \times 10^{-6} \\ \mathrm{t}_{\mathrm{L}} & 1.0 \times 10^{-6}+(\mathrm{N}-1) .7 \times 10^{-6} \\ \mathrm{t}_{\mathrm{C}} & 3.5 \times 10^{-6}+(\mathrm{N}-1) 2.3 \times 10^{-6} \\ \mathrm{t}_{\mathrm{I}} & 4.4 \times 10^{-7} \mathrm{~N} \\ \mathrm{t}_{\mathrm{V}} & 6.7 \times 10^{-7} \mathrm{~N}\end{array}$

Equation 5.30 reduces to

$$
\mathrm{T}_{\mathrm{PR}} \approx 4 \times 10^{3} \mathrm{t}_{\mathrm{f}}+3.28 \times 10^{-2} \mathrm{M}_{\mathrm{s}}+4 \times 10^{-3} \sum_{1=1}^{\mathrm{M}_{s}} \frac{4 \cdot 1 \mathrm{Q}_{1}+0.335}{\mathrm{~N}_{1} \mathrm{~h}_{1}}
$$

The value of $\mathrm{N}_{1} \mathrm{~h}_{1}$ will in general be greater than ${ }^{5 P_{1}}$ for initial surfaces. However as surfaces progress this value will decrease since splitting will cause more particles in the more important classes. Assuming the very conservative value $N_{1} h_{1}=.1 P_{1}$ reduces Equation 5.31 to

$$
T_{P R} \approx 4 \times 10^{3} t_{f}+3.28 \times 10^{-2} M_{S}+10^{-5} \sum_{i=1}^{M_{S}}\left(4.1 Q_{i}+0.335\right)
$$

The value of $Q_{1}$ will seldom be over 1 (for any problem requiring variance reduction) and therefore the third term can be ignored compared with the second resulting in

$$
\mathrm{T}_{\mathrm{PR}} \approx 4 \times 10^{3} \mathrm{t}_{\mathrm{f}}+3.28 \times 10^{-2} \mathrm{M}_{\mathrm{s}}
$$


Although Equation 5.33 is only a very rough approximation it is still useful as an indicator of the amount ot: time spent for learning surfaces. Furthermore, the assumptions have been made in such a manner so as to overestimate $T_{P R}$. Choosing any reasonable value for $t_{f}\left(10^{-4}\right.$ to $10^{-6}$ ) and $M_{s}(<10)$ and allowing for approximation errors of a factor of ten still results in a $T_{P R}$ on the order of 10 seconds. For Monte Carlo runs of many minutes this time is quite small compared with the time saved by variance reduction.

\subsection{3) Effectiveness}

Geometry splitting and energy splitting techniques have proven to be quite effective rechniques for a great variety of Monte Carlo problems. Since state space splitting is closely related to these techniques the effectiveness of learning state space splitting surfaces by pattern recognition is described relative to conventional techniques. This results in the relation

$$
\begin{aligned}
& E_{R}=\frac{N_{e} \Delta t}{N_{e}^{*} \Delta t_{e}^{*}} \\
& \text { where } E_{R}= \text { effectiveness of state space splitting using } \\
& \text { surfaces learned by pattern recognition } \\
& N_{e}= \text { number of source particles required to achieve } \\
& \text { the desired relative error using state space } \\
& \text { splitting }
\end{aligned}
$$




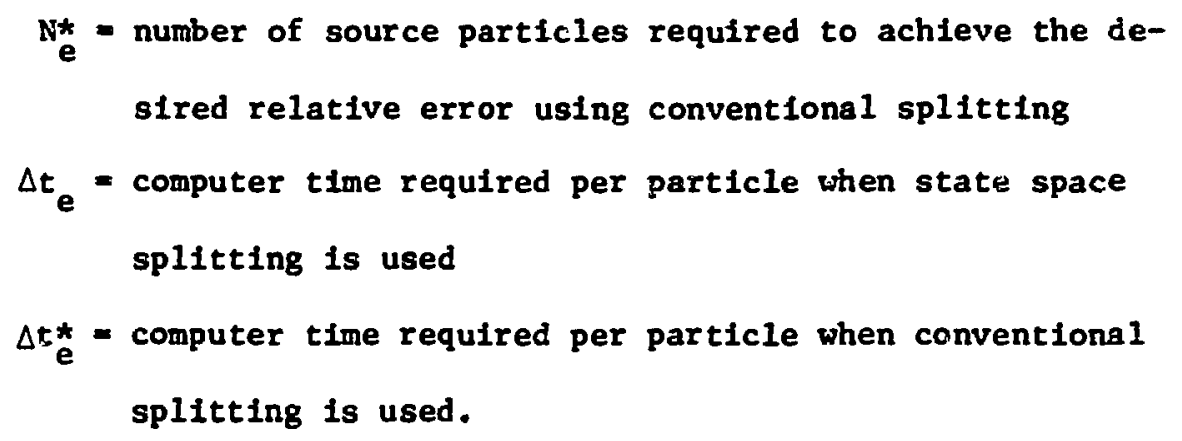

If $t_{B}$ is the computer time required per particle to perform the Monte Carlo calculations without any form of splitting then

$$
\begin{aligned}
& \Delta t_{e}^{*}=\Delta t_{g}+\Delta t_{B}=\Delta t_{1}+\Delta t_{2}+\Delta t_{3}+\Delta t_{B} \\
& \Delta t_{e}=\Delta t_{s}+\Delta t_{B}+\frac{T_{P R}}{N_{e}}=\Delta t_{4}+\Delta t_{1}+\Delta t_{B}+\frac{T_{P R}}{N_{e}}
\end{aligned}
$$

where $\Delta t_{g}, \Delta t_{1}, \Delta t_{2}, \Delta t_{3}$ are defined by Equation 5.13 . $\Delta t_{s}, \Delta t_{4}$ are defined by Equation 5.15 . $T_{P R}$ is given by Equation 5.29 .

Substituting Equations 5.35 and 5.36 into Equation 5.34 results in

$$
E_{R}=\frac{N_{e}\left(\Delta t_{4}+\Delta t_{1}+\Delta t_{B}\right)+T_{P R}}{N_{e}^{*}\left(\Delta t_{3}+\Delta t_{2}+\Delta t_{1}+\Delta t_{B}\right)}
$$

The amount of variance reduction is indicated by the ratio

$$
R=N_{e} / N_{e}^{*}
$$


which measures how much more efficlent for reducing the variance state space splitting is than conventional splitting. The values of $N_{e}$ and $\mathrm{N}$ e can be further reduced to

$$
\begin{aligned}
& N_{e}^{*}=N_{A}-\Delta N_{C} \\
& N_{e}=N_{A}-\Delta N_{S}+\Delta N_{L}
\end{aligned}
$$

$$
\text { where } \begin{aligned}
\mathrm{N}_{\mathrm{A}}= & \text { number of source particles required to achieve } \\
& \text { the desired relative error if neither conven- } \\
& \text { tional nor state space splitting is used } \\
\Delta \mathrm{N}_{\mathrm{C}}= & \text { reduction of } \mathrm{N}_{\mathrm{e}} \text { due to variance reduction when } \\
& \text { using conventional splitting } \\
\Delta \mathrm{N}_{\mathrm{S}}= & \text { reduction of } \mathrm{N}_{\mathrm{e}} \text { due to variance reduction when } \\
& \text { using state space splitting, assuming the split- } \\
& \text { ting surfaces are known prior to the Monte } \\
& \text { Carlo calculation. } \\
\Delta \mathrm{N}_{\mathrm{L}}= & \text { increase in } \mathrm{N}_{\mathrm{e}} \text { due to the learning of split- } \\
& \text { ting surface. }
\end{aligned}
$$

If the state space splitting surfaces are known prior to the Monte Carlo calculation $\left(\Delta \mathrm{N}_{L}=0\right)$, then in general $R<1$. This is because state space splitting includes the advantages of conventional splitting (i.e., $\Delta \mathrm{N}_{\mathrm{C}}$ ) as well as the additional advantages of splitting in time and direction space (i.e., $\Delta N_{S}>\Delta N_{C}$ ). Through proper feature selection optimal combinations of state space parameters can be used which further 
increases $\Delta \mathrm{N}_{\mathrm{S}}$.

When splitting surfaces mist be learned, the value of $\left(\Delta N_{S}-\Delta N_{L}\right)$ varies throughout the calculation starting at zero and increasing to $\Delta N_{S}$ for which splitting surfaces are known a priori. In this case $R$ could possibly be $>1$ for some Monte Carlo problems; however, by using decision surfaces for splitting before they have converged, $\Delta N_{L}$ can be greatly reduced. Monte Carlo problems exist for which conventional splitting is inadequate; whereas state space splitting appears to be quite attractive (such problems are now usually split into two separate problems where the tally from one provides the source to the other). For this type of problem $R<1$. If several similar Monte Carlo problems are being calculated, splitting surfaces can be learned in the first problem and used in the others, thus making $\Delta N_{L}=0$ for subsequent similar problems. The value of $\Delta \mathrm{N}_{\mathrm{L}}$ depends on the amount of learning performed per source particle which in turn depends on the number of prototypes created per source particle, $\mathrm{N}_{1}$ (see Section 5.3.2). Since prototypes are created at collisions, problems in which the particles have a high number of collisions before leaving the system result in converged splitting surfaces after fewer source particles. This point is illustrated in Chapter IV by comparing the number of source particles until convergence (relative to each problem) for the problems described in Sections $4 \cdot 2.3$ and 4.4 .3 : 


\section{Collistons/Source Particle}

$(4.2 .3)$

$(4.4 .3)$
100

12
No. Source Particles

until Convergence

40

300

Problems which do not have a large number of collistons are often problems which do not require much variance reduction if any. If a problem containing few collisions requires varlance reduction, the learning technique can be modified to produce prototypes at surface intersections as well as collisions. However, in general problems containing many collisions are the most suitable for learning state space splitting surfaces. For many problems $\Delta t_{B}$ will be much greater than the other time parameters $\Delta t_{1}, \Delta t_{2}, \Delta t_{3}, \Delta t_{4}$ and $T_{P R} / N_{e}$ thus reducing Equation 5.37 to

$$
E_{R} \approx \frac{N_{e}}{N_{e}^{*}}=R
$$

For problems in which this is not true, the time parameters of Equation 5.37 must be taken into consideration. Since these parameters and $R$ depend upon many problem characteristics and user supplied input, it is ludicrous to propose that $E_{R}$ will always be less than 1.0 . However, from the results and analysis of this chapter and Chapter IV, it does appear that for many problems, this will be the situation. Only a great number of computational experiments using a general purpose Monte Carlo code will provide the data necessary to define for which problem types $E_{R}<1$. 


\section{4) Limitations of this Research}

The research described in this thesis represents an initial investigation into the area of pattern recognition applied to state space splitting surface identification. The following sub-sections include the more important limitations of this research and the learnIng of state space surfaces.

\subsection{1) Classifier Selection}

Both the deterministic and statistical classifiers investigated in Chapter IV are of the same construction in that they assume a linear form of $g(Y)$. It is very unlikely that any optimal splitting surface In state space having overlapping distributions will be linear. It has been assumed that in most cases a linear surface is a sufficient approximation to adequately split particles. Furthermore, feature space can be altered to improve this approximation. Experience with the technique will be the ultimate test in determining whether or not linear discriminant functions are sufficient.

There are many different types of classifiers for Case $D$ and $E$ data (see Section 3.1.2), for example, the expansion given by Equation 3.4. Some other technique may prove more attractive for classifying the types of distributions resulting from Monte Carlo calculations. In Chapter IV it was found that the statistical classifier used is hindered by its accumulated overshoot and that the deterministic classifier has 
the characteristic that for overlapping $p\left(C_{1} \mid Y\right)$, the variability never goes to zero. An attractive alternative might be a hybrid classifier that uses deterministic classification during the initial approach and then uses statistical classification until the final convergence criterion is met.

\subsection{2) User Input}

One of the incentives for using pattern recognition to identify splitting surfaces is to relieve the user of the burden of providing a priorl information to the Monte Carlo calculation. However, as Is described in Section 5.2 the user must provide the values of $f_{i}$, $u_{i}^{\min }, u_{i}^{\max }$, and $d_{i}$ for the feature selector (see Figure 5.4). Once the $f_{i}^{\prime}$ 's have been selected, the values of $u_{i}^{m i n}, u_{i}^{\max }$, and $d_{i}$ are determined and can be easily supplied. The main problem then is selecting a good cholce for the $f_{1}$ This cholce will have to be based upon intuition and experience using heuristics similar to those described in Section 5.2. Although certainly a non-trivial operation, the above selection is a qualitative decision for which humans are much more suited than computers. Simllarly, the learning of a splitting surface using the $f_{i}$ is a quantitative dectsion for which the computer is much better sulted. In conventional splitting the user is required to perform both these tasks.

There is other input which could be made optional as user input and includes: 
(1) Initial selection of $\mathrm{W}$

(2) Convergence criterla for the variability and misclassification rate

(3) A choice for $R$ (see Equation 5.2)

(4) The limit on the number of splitting surfaces, $M_{s}$ (see Section 5.3.2)

(5) For multiple tallies, the values of $p_{1}$ (see Equation 5.5)

(6) For multiple point detector tallies, the values of $m_{i}$ (see Equation 5.8)

It would be a little presumptuous to expect the general Monte Carlo user to input all this additional information. The default values can be chosen as described in Sections 5.1 and 5.2; however, after experience has been gained with the technique better values will undoubtedly be found.

\subsection{3) Range of Application}

As with any variance reduction technique this one has the problem that the user is not certain when to use it. The result is that problems not requiring splitting or for which splitting will not help may be slowed down. This technique does have the advantage that by usIng the $\mathrm{F}_{2}$ test (see Equation 5.2), the code will stop learning surfaces if they are not needed. However, this test is made only after the first splitting surface has been learned. 


\section{5) Summary}

A systematic approach has been described in section 5.1 for collecting prototypes, determining classification, and managing the selection of splitiing surfaces. The feature selection process can be performed by a user-supplied subroutine containing the information shown in Figure 5.5. The selection of the features $\left(f_{i}\right)$ is the most important user input and must be based on the intuition or experience of the user. Recommended heuristics have been given to aid in this selection. Although an absolute statement cannot be made about the timing and effectiveness, by using constants derived in this research and assuming reasonable values for problem parameters, it does appear that the technique should be quite attractive compared with conventional splitting techniques. 
VI. Conclusions and Recommendations

In Section 1.4 four steps toward demonstrating "proof of prirciple" were stated. These steps are repeated below along with the conclusions drawn from this research.

(1) Develop a pattern recognition system that can be used to learn splitting surfaces in Monte Carlo transpert calculations.

A scheme has been designed whereby fattern recognition can be used to learn splitting surfaces in state space. Training sets or prototypes are required before the splitting surface can be learned. These prototypes consist of points in state space (collision points were used in this rasearch) with their corresponding importances. The importances are determined by measuring the probability that a particle leaving a given point contributes to the Monte Carlo tally under consideration. Both statistical and deterministic classifiers learn splitting surfaces satisfactorily. From the examples of this research it appears that the number of source particles required to learn a surface is small enough to make the technique useful for practical applications.

(2) Investigate the performance of statistical and deterministic classifiers when used to recognize splitting surfaces. Analyze the sensitivity of the parameters involved.

The deterministic classifier using the fractional correction rule is superior to the statistical one with respect to per- 
formance, computer time, sensitivity to the learning parameter, and sensitivity to initial conditions. The learning parameter, $\lambda$, affects the rate of convergence of the splitting surface. Although the optimum choice of a value for $\lambda$ depends upon the characteristics of the Morte Carlo problem under investigation, it was found that a single $\lambda(\lambda=.05)$ can be used with the deterministic classifier without seriously penalizing convergence. The convergence is also affected by the initial guess of the splitting surface location. Initial splitting surfaces should be located in the least important region of state space. Each component, $y_{i}$, of feature space should be normalized to the interval from zero to one and should be selected so that as $y_{i}$ increases, the importance decreases. As a result the least important point in feature space is given by $\mathrm{Y}=\left[\mathrm{y}_{1}, \mathrm{y}_{2}, \ldots, \mathrm{y}_{\mathrm{N}}\right]=[1,1, \ldots, 1]$.

(3) Propose a system for applying pattern recognition to a general purpose Monte Carlo code.

A system for implementing pattern recognition techniques in a general purpose Monte Carlo code is given in Chapter V. The, scheme requires the user to supply the information needed by the feature selector. Other parameters can be supplied by the user or by default values. Selection of these values must be gained from experience with the technique. 
(4) Analyze the effectiveness that can be expected by using pattern recognition as a variance reduction technique.

The effectiveness of the technique is problem dependent and user input dependent. The parameters involved have been identified and values have been determined for those parameters which are independent of the Monte Carlo problem or the user input. Based upor these values and reasonable choices for the other parameters, it appears that the technique should be more useful than conventional splitting. The problem scope and degree of effectiveness can only be determined by applying the technique to many Monte Carlo problems using a multi-purpose code.

Based upon these conclusions, it is recommended that a multi-purpose Monte Carlo code such as MCN at the Los Alamos Scientific Laboratory be modified to split in state space instead of the presently used splitting. Having done this the system described in Chapter $V$ can be included to provide the splitting surfaces. The deterministic classifier described in this research can be used directly except that an allowance must be made for variable dimensioned feature vectors. The modified code should be used on a range of Monte Carlo problems to determine many of the unknown problem related parameters described in this research and to determine the success of the technique for reducing computer time. The code should then be used routinely to determine user related problems and to gain experience with the technique. Only after such an investigation can the success of the tecinique be determined. 


\section{Acknowledgements}

I wish to thank Dr. Billy $\mathrm{V}$. Koen for his continual guidance as my supervising professor during both my M.S. and Ph.D. studies. Through his intellectual curiosity and professional honesty, Dr. Koen has been a strong influence on my engineering education as well as my philosophical views. I wish to also thank my other comittee members at the University of Texas, Dr. E. Linn Draper, Dr. Baxter F. Womack, Dr. Robert N. Little, and $\mathrm{Dr}$. Joe 0. Ledbetter for their help with this dissertation.

I am very grateful to the many people at the Los Alamos Scientific Laboratory (LASL) who made this research possible. In particular I wish to thank Dr. Edmond D. Cashwell for making it possible for me to perform this research at IASL and for the many hours he has spent reading and discussing this dissertation with me. I consider it an honor to have a man of Dr. Cashwell's capabilities on my comittee.

This research was initially supported by the Atomic Energy Commission by the award of a Traineeship in Nuclear Science and Engineering administered by the University of Texas at Austin. Prel1minary computer calculations were performed at the University of Texas Computation Center. The calculations and results reported in this dissertation were performed at the Los Alamos Scientific Laboratory Central Computer Facility. 


\section{Azpeadix A. Yonte Carlo Sampling for \\ Viejezon Iransoort}

The furpose of tisis Appendix is to give the reacer a "physical feel" for the processes involved in using the "'onte Carlo Eethod for neutron transport. It is not the purpose of this nopendix to derive and prove the atheaticai basis of Yonte Carlo. The structure of this description of Icnte Cario is modeled after reference 7 with rany of the ideas taken fron rejerence $3 \div$. These references should be consulted if Eore datails are desired. Section A.I presents the basic principles of Yonte Carlo as used for zeutron transport. The remaining sections treat iccioicual topics of neutron transport and give examples of how the pisysics of the problem is programed into a statistical =oiel.

\section{A.1 Basic Príciores}

The basic problea of neutron transport consists of calculating the number of neutrons that are emitted from a source, undergo any number of collisions with atozic nuclei, and finally arrive at some region of state space (cefined below) of interest to the person trying to solve the transport problem. The term state space refers to the complete description of the physical properties of the neutrons as given by: 


$$
(x, y, z, u, v, w, E, t)
$$

where $x, y, z$ are the coordinates of the neutron in a Cartesian Coordinate System (see Figure A.1)

$u, v, w$ are the direction cosines of the neutron with respect to the $x, y$, and $z$ axis (see Figure A.1)

$E$ is the kinetic energy of the neutron

$t$ is the time elapsed since the process under study has begun

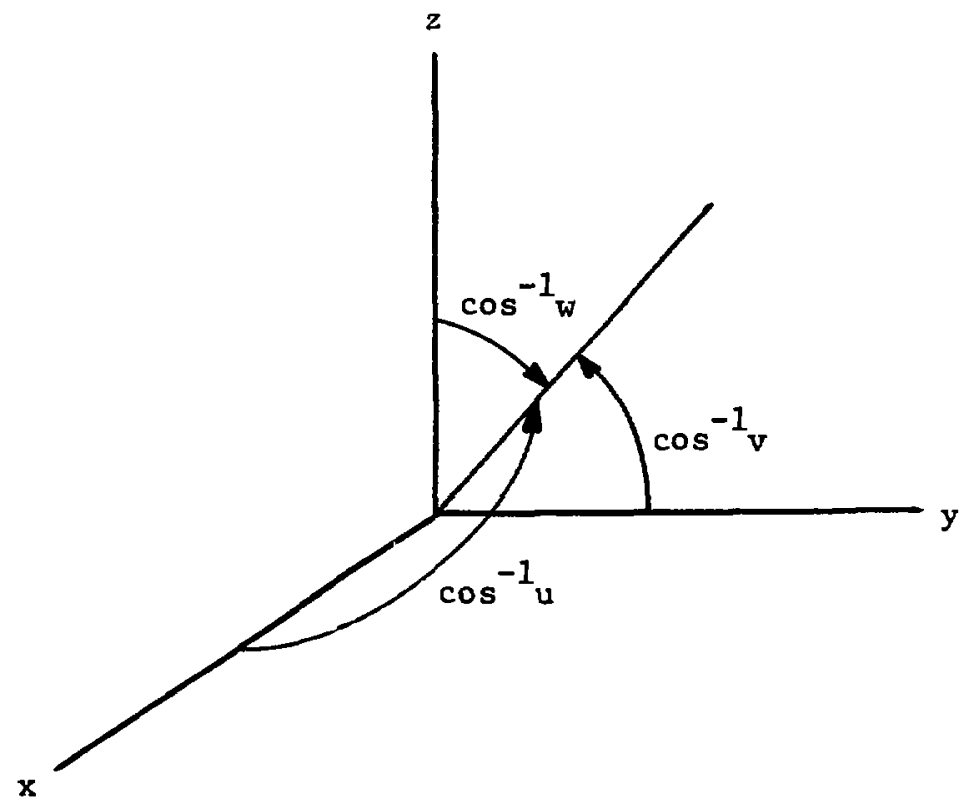

Figure A.1 Geometry Coordinates

Most methods used to solve the transport equation treat all the neutrons as a whole (i.e., they solve for the flux of neutrons) and then 
proceed to find the value of the flux at all points in state space. The Monte Carlo method works quite differently in that neutrons are treated one at a time independently of the others. After calculating the behavior of many individual neutrons, the average or mean behavior of the neutron "sample" is determined. This mean value is used as an approximation of the value that would be obtained if an infinite number of neutrons (i.e., the total population) had been treated. Corresponding to this mean estimate is a probabilistic error based upon the statistical behavior of the neutrons.

Neutron transport is ideally suited for the Monte Carlo method since most of the physical processes involved are probabilistic in rature. For example:

(1) a neutron undergoes a collision per unit path length with probability $\Sigma_{t}$.

(2) the probability of a particular nuclear reaction, $i$, occurring during a collision with a nucleus is $\sigma_{i} / \sigma_{t}$.

(3) the probability of scattering at an angle $\theta$ (in the center of mass system)' is determined by $\sigma(\theta)$.

(4) the probability per unit time that a nucleus decays is given by the decay constant $\lambda$.

(5) the probability of a prompt fission neutron being emitted with energy between $E$ and $E+d E$ is given by $\chi(E) d E$. 
Figure A.2 illustrates the major operations performed by a Monte Carlo program when used to follow neutrons through state space. These operations are introduced below and described in more detail in the following sections.

(1) Source (Section A.2). The source of neutrons is usually described as a user supplied subroutine in a Monte Carlo program. It is the purpose of this subroutine to describe the initial state space parameters $(x, y, z, u, v, w, E, t)$.

(2) Collision or Escape Decision (Section A.3). The geometry of any Monte Carlo problem is divided into regions or cells which may or may not correspond to material regions. This decision process consists of "sampling" the probability of having a collision as determined by $\Sigma_{t}$ of the material in the given region. It is for this decision that the majority of geometry calculations are made since the distance from the present $(x, y, z)$ to the next intersection with the cell boundary must be known.

(3) Entering a New Region (Section A.4). Th1s process consists of determining which region the particle is entering after leaving the present region.

(4) Collision Type (Section A.5). The process for determining which type of reaction occurs is determined by the relationship of the respective cross sections. 


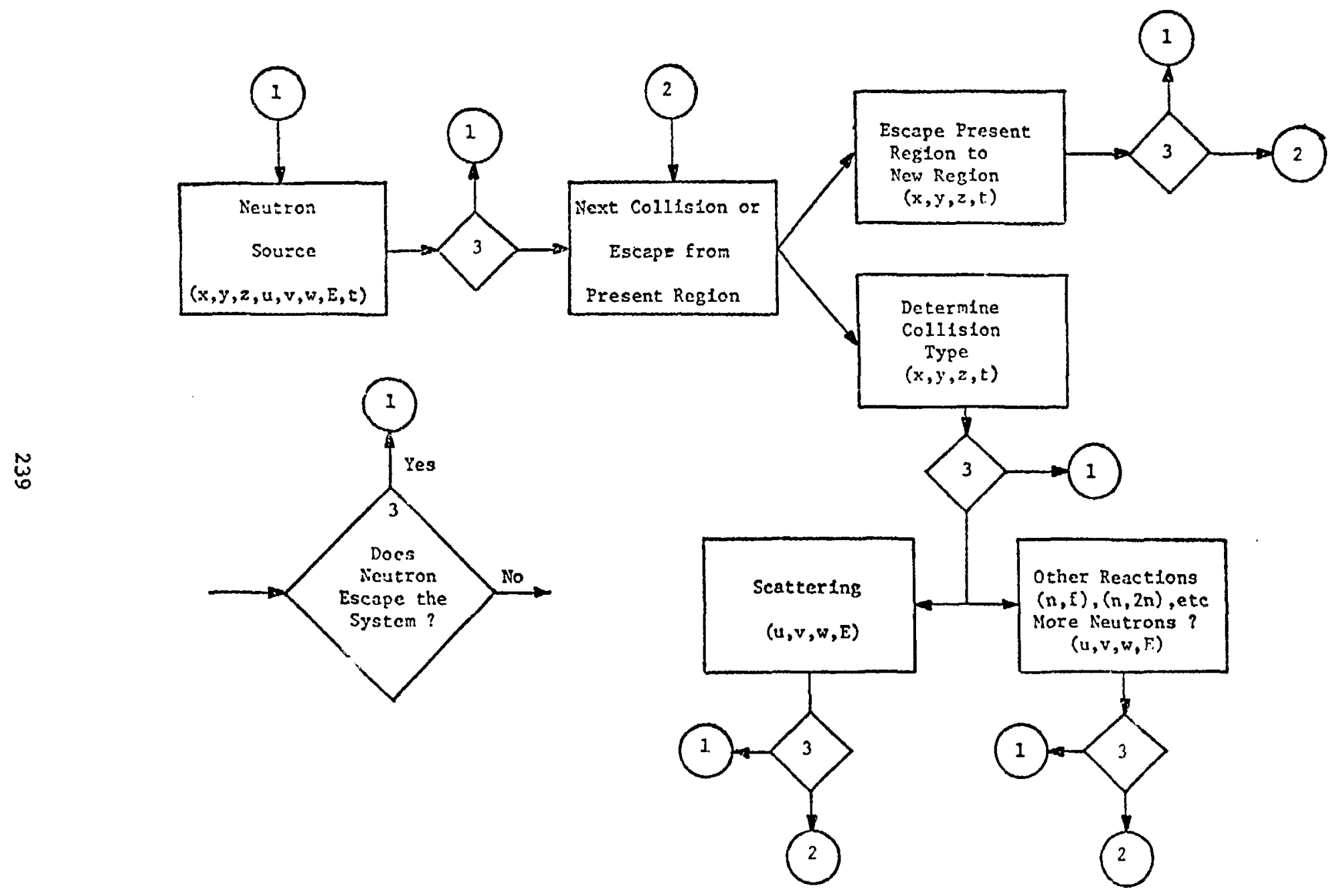

Figure A.2 Major Monte Carlo Operations for Neutron Transport 
(5) Scattering (Section A.6). Both elastic and inelastic scattering result in a change in energy which is related to the scattering angle (usually given in the center of mass system). The scattering decision consists of sampling for this scattering angle. The new neutron energy and direction cosines can then be determined from this angle.

(6) Other Reactions (Section A.7). Many other reactions can occur (i.e., fission, $[n, 2 n]$, etc.) and often lead to the creation of additional neutrons. In such a case an energy $E$ and direction $(u, v, w)$ must be determined for each neutron emitted. In addition, for fission reactions the number of neutrons emitted can be sampled or a: average value used.

(7) Tallies (Saction A.8). The tally of a Monte Carlo transport problem consists of the "answer" that one is seeking as determined by the mean behavior of the neutron historles followed. Examples of such tallies are: current crossing a particular surface, flux at a point, number of captures in a region, etc. Because of the generality involved, the information necessary to determine the tally can be extracted from any part of the Monte Carlo calculation. Therefore, the tally operation is not shown in Figure A.2. 
The following sections frequently refer to the cholce of a random number, $r$, for sampling probability distributions. The mathematical description of "random" can be found in nany of the references $^{7,24, ? 9}$. For the purpose of this description it is assumed that the random number, $r$, has an equal. probability of falling between the value of 0 and 1 and that all subsequent random numbers are uncorrelated $^{14,29}$.

The coordinate system used in the following description is as shown in Figure A.I where the angles are in the laboratory system unless otherwise noted. Energy is also relative to the laboratory system.

\section{A.2 The Neutron Source}

The sampling of a general neutron source involves the following operations:
(1) sampling of the space coordinates $(x, y, z)$
(2) sampling of the direction cosines $(u, v, w)$
(3) sampling of the energy spectrum
(4) determination of the time

The above operations are performed independently and will be described in this section by using several examples. In these examples all sampling will be performed uniformly over the quantities (surface, volume, etc.) of interest. 


\section{A.2.1 Space Coordinates}

Two examples of sampling the space coordinates $(x, y, z)$ are described in this section: (1) a flat two dimensional surface and (2) a right circular cylinder. Although these examples comprise only a small fraction of the possible sources, the techniques used to sample are similar for all sources and thus provide the reader with a "feel" for the processes involved.

In many Monte Carlo problems neutrons are emitted from a flat two dimensional surface. Examples of such sources are cross sections of a neutron beam, the side of a rectangular reactor, a spill of radicactive liquid on a flat surface, etc. Three examples are shown in Figure A.3 - a rectangle centered at the origir, a circle centered at the origin, and a general function $f(y, z)$.
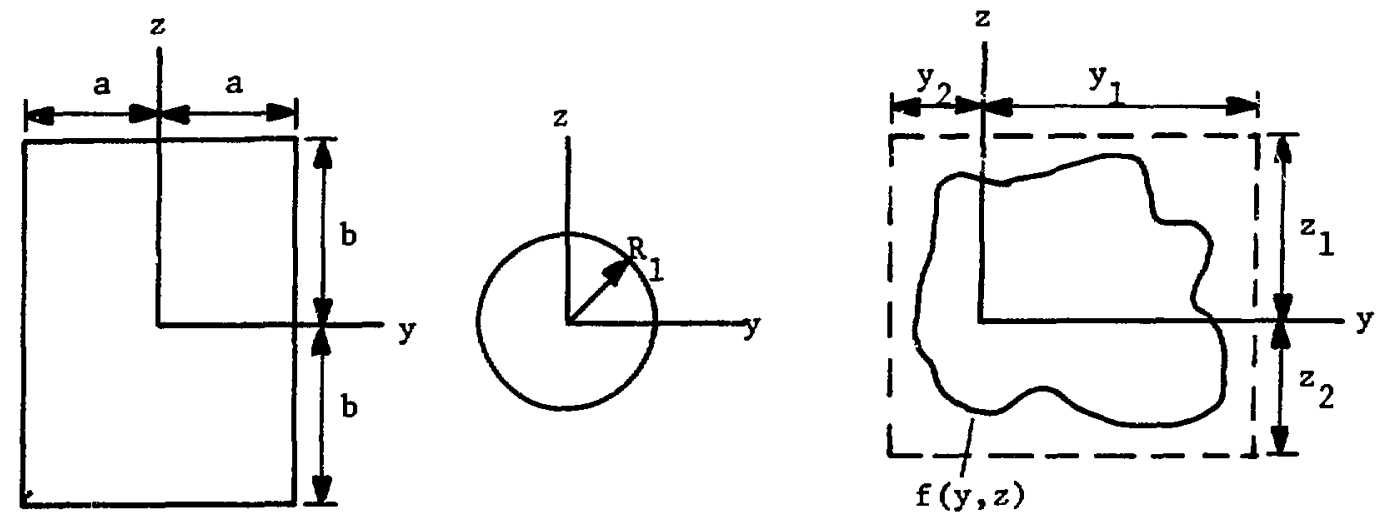

Figure A.3 Sampling Two-Dimensional Sources 
The rectangular source is the easiest to sample and consists of :

(1) choosing a point randomly along the y axis between $y=-a$ and $y=+a$ and

(2) choosing a point randomly along the $z$ axis between $z=-b$ and $z=+b$.

This is done by performing the operations shown in Figure A-4 which results in uniform sampling over the area of the rectangle.

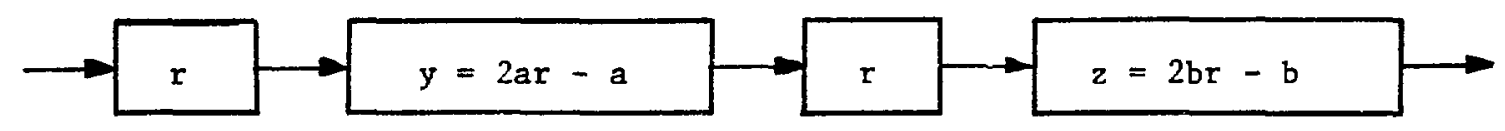

Figure A.4 Sampling a Rectangular Two Dimensional Source

The sampling of the circle is facilitated by using polar coordinates as shown by Figure A.5. This is done since one wishes to sample evenly over the area of the circle and the area of a circle is more easily defined by polar coordinates. 


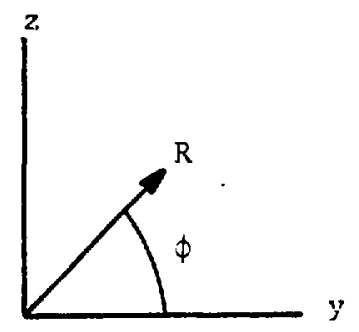

Figure A.5 Coordinates Used for Sampling a Circle

The sampling of the circle consists of:

(1) choosing a $R$ randomly between $R=0$ and $R=R_{1}$ (actually $R^{2}$ is chosen randomly between $R^{2}=0$ and $R^{2}=R_{1}^{2}$ )

(2) choosing a $\phi$ randomly between $\phi=0$ and $\phi=2 \pi$

(3) converting polar coordinates $(R, \phi)$ to rectangular coordinates $(y, z)$

These operations are performed as shown by Figure A.6.

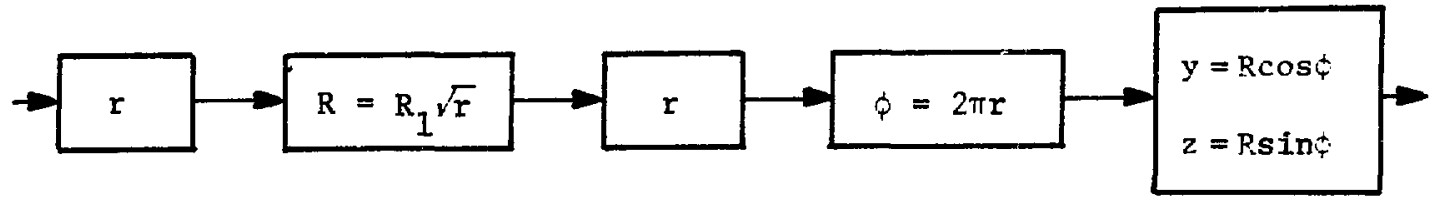

Figure A.6 Sampling a Circular Two Dimensional Source 
The sampling of the general function $f(y, z)$ is usually best solved by the method of "rejection". This consists of defining a rectangular area completely enclosing $f(y, z)$ and:

(1) sample the rectangle the same as for the earlier case resulting in a $\left(y^{\prime}, z^{\prime}\right)$

(2) test to see if this $\left(y^{\prime}, z^{\prime}\right)$ lies within the area defined by $f(y, z)$. This is done by checking the sign of $f\left(y^{\prime}, z^{\prime}\right)$. [assuming that $\left(y^{\prime}, z^{\prime}\right)$ which are inside $f(y, z)=0$ result in $\left.f\left(y^{\prime}, z^{\prime}\right)<0\right]$

(3) If $\left(y^{\prime}, z^{\prime}\right)$ does not lie within the area defined by $f(y, z)$ then repeat the above steps, otherwise use the value of $\left(y^{\prime}, z^{\prime}\right)$.

Figure A.7 1llustrates this process.

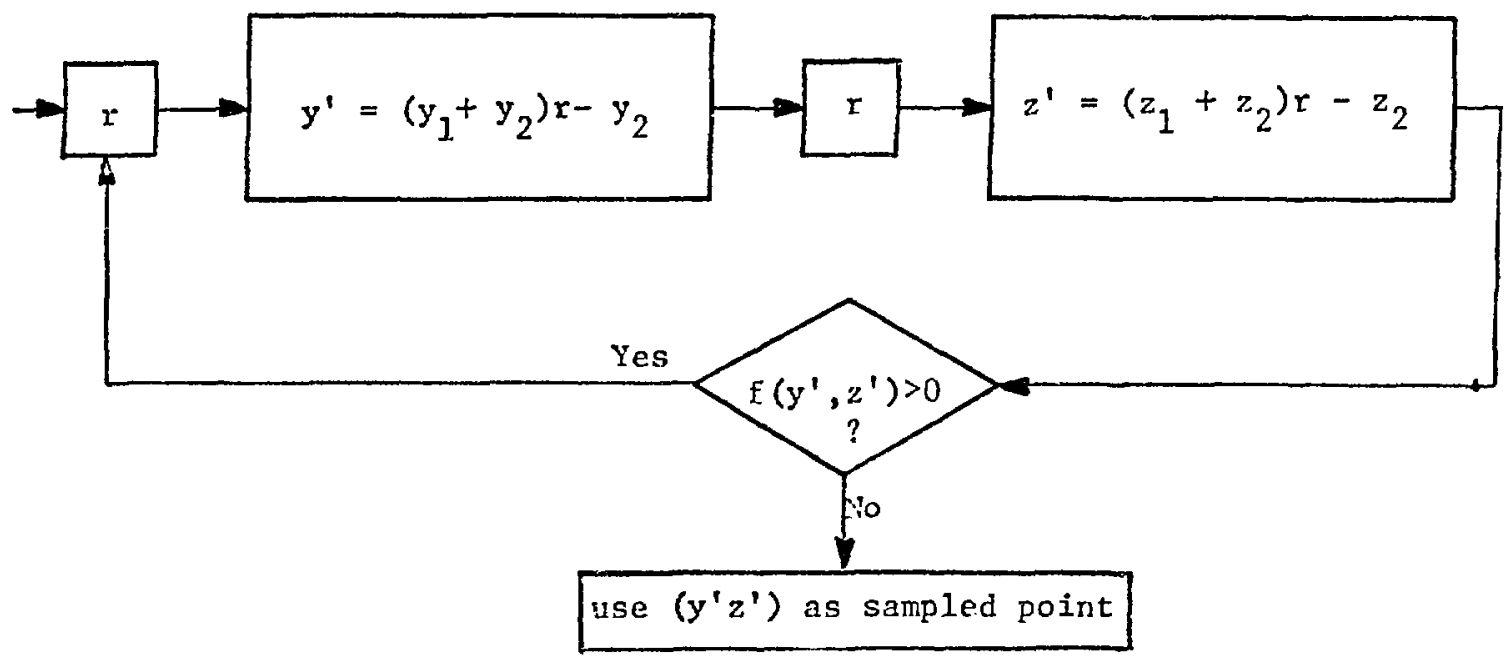

Figure A.7 Sampling by the Refection Technique 
A source within a right circular cylinder (see Figure A.8) is frequently encountered in reactor related problems dealing with cylindrical fuel rods and pellets. This probiem consists of sampling a volume as opposed to an area in the earlier examples.

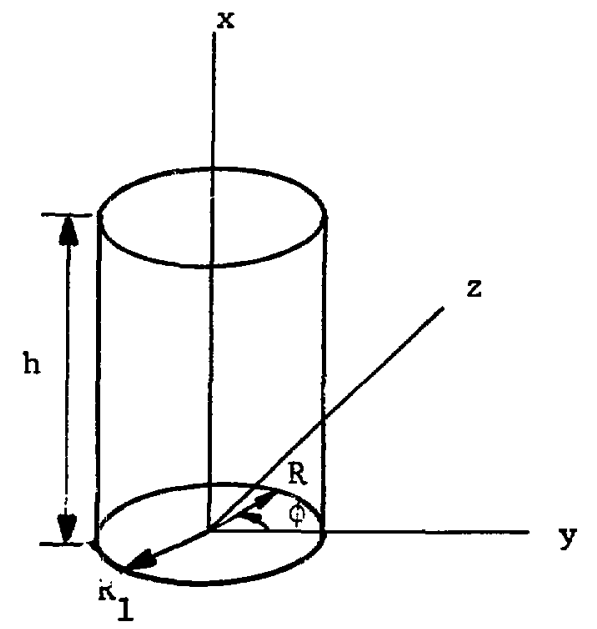

Figure A.8 Right Circular Cylinder

Once again cylindrical coordinates are preferred as shown by

Figure A.5. A $\phi$ and $R$ are selected as giver by Figure A.6 followed by the selection of $x$ (see Figure A.9). 


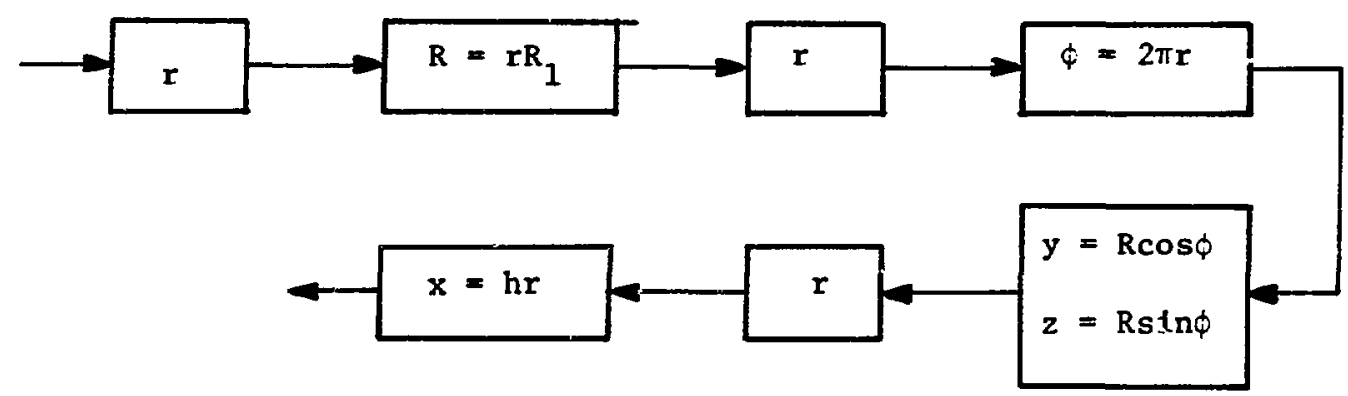

Figure A.9 Sampling the Right Circular Cylinder

\section{A.2.2 Direction Cosines}

This section considers two different sources: (1) a directed beam and (2) an isotropic source. The directed beam as shown by Figure. A.10 has the trivial solution

$$
\begin{aligned}
\mathbf{u}=\mathbf{w} & =0 \\
\mathbf{v} & =1 .
\end{aligned}
$$

Since each neutron has the same direction there is no distrioution to sample. 


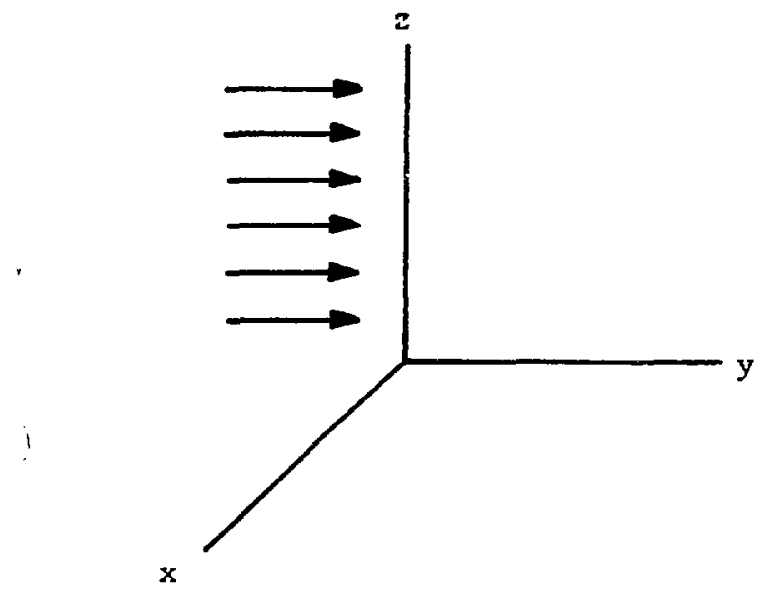

Figure A.10 Ths Directed Beam Source

The problem of selecting a $(u, v, w)$ for an isotropic source is equivalent to that of chonsing a point $(u, v, w)$ uniformly distributed on the unit sphere $u^{2}+v^{2}+w^{2}=1^{7} \quad$ (see Figure A.11).

The unit area on this surface is given by $\sin \theta d \theta d \phi$. This distribution can be sampled by the following operations (see Figure A.12):

(1) select a w randomly between -1 and +1

(2) determine $\rho$ (see Figure A.11)

(3) select a $\phi$ randomly between 0 and $2 \pi$

(4) convert from coordinates $(\phi, \rho)$ to $(u, v)$ 


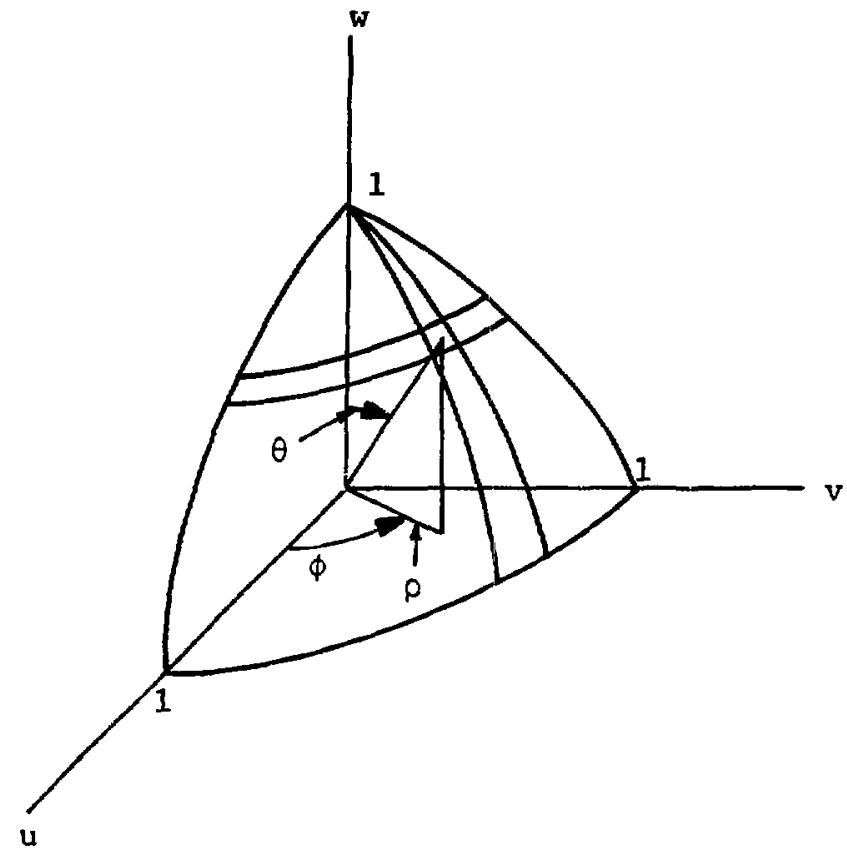

Figure A.11 The Isotropic Source

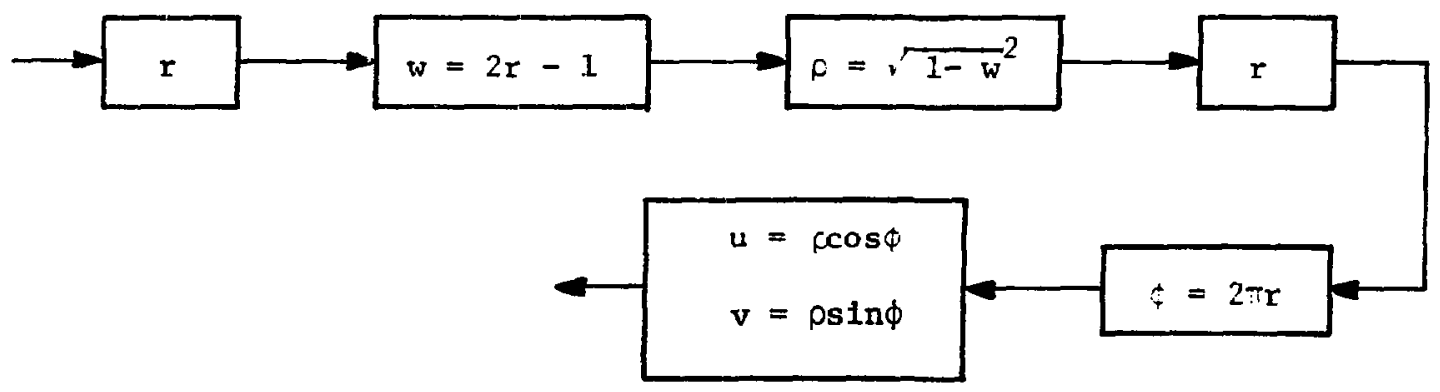

Eigure A.12 Sampling an Isotropic Source 


\section{A.2.3 Energy Distribution}

The energy of source neutrons is frequently a single value for all particles and thus has no distribution. The fission spectrum is used for some sources and is discussed in section A.7. Many times experimental data results in an energy distribution which consists of the number of neutrons lying within specified energy intervals

$$
\begin{aligned}
& N\left(E_{1}, E_{i+1}\right)= \text { number of neutrons having energy between } \\
& E_{1} \text { and } E_{i+1} \text { where } i=0, \ldots M-1 \\
& \text { and } E_{0}=\text { minimum energy of neutrons } \\
& E_{M}=\text { maximum energy of neutrons }
\end{aligned}
$$

In such a case the data must first be normalized by dividing by the total number of neutrons

$$
p\left(E_{1}, E_{1+1}\right)=\frac{N\left(E_{1}, E_{1+1}\right)}{\sum_{i=0}^{M-1} N\left(E_{i}, E_{i+1}\right)}
$$

which results in the probability of a source neutron having an energy between $E_{i}$ and $E_{i+1}$. The probability distribution is given by summing the $p\left(E_{1}, E_{i+1}\right)$ as given by

$$
P\left(E_{i+1}\right)=\sum_{j=0}^{i} p\left(E_{j}, E_{j+1}\right)
$$


where $P\left(E_{M}\right)=1.0$. A random number is then selected between 0 and 1.0 and if

$$
\begin{gathered}
P\left(E_{i}\right)<r \leqslant P\left(E_{i+1}\right), \quad \quad i=0, \ldots M-1 \\
\text { where } P\left(E_{0}\right)=0.0,
\end{gathered}
$$

then the energy of the neutron lies between $E_{i}$ and $E_{i+1}$. The energy can be further specified by linearly interpolating between $P\left(E_{i}\right)$ and $P\left(E_{i+1}\right)$ (see Figure A.13) resulting in

$$
E=E_{1}+\frac{\left(r-P_{1}\right)\left(E_{2}-E_{1}\right)}{\left(P_{2}-P_{1}\right)}
$$

where $r$ is the same random number used in equation A.4.

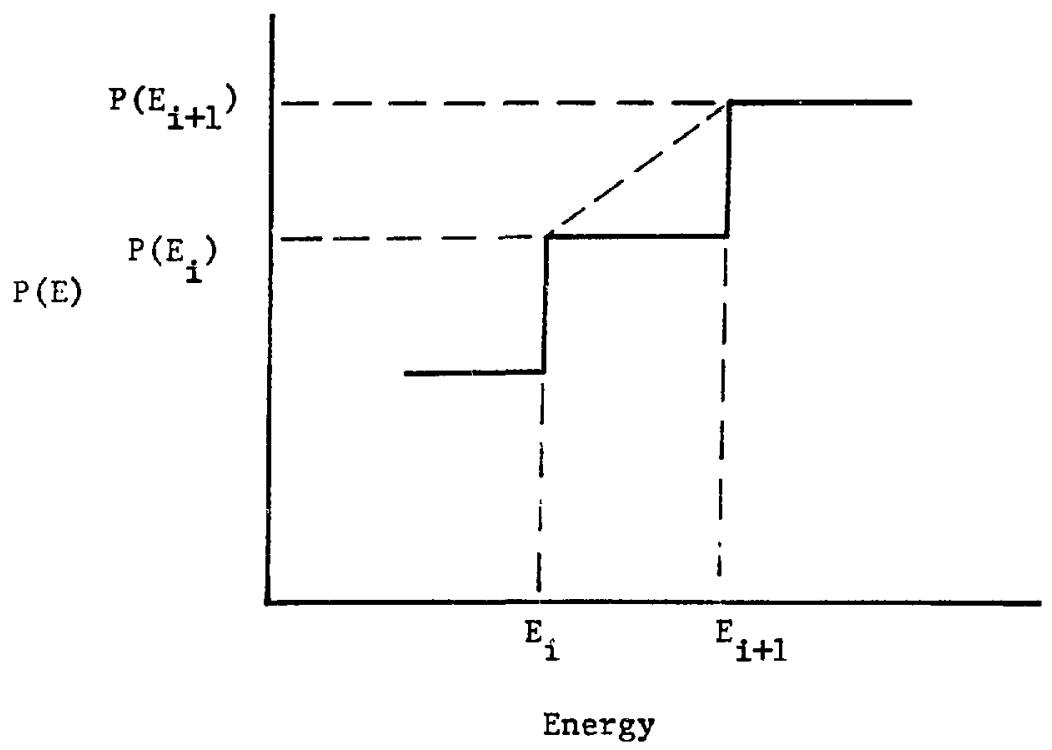

Figure A.13 Linear Interpolation to Determine the Energy 


\section{A.2.4 Time}

Source neutrons are generally considered to be emitted at time $t=0$; however, there are instances in which the source is time dependent. An example of this is the emission of delayed neutrons which are emitted as

$$
\operatorname{dn}(t)=\lambda \mathrm{n}_{0} e^{-\lambda t} \mathrm{dt}
$$

where $\operatorname{dn}(t)=$ the number of neutrons emitted between

$$
\text { time } t \text { and } t+d t
$$

$$
\begin{aligned}
& \mathrm{n}_{0}=\text { the number of precursor nuclei at time } \\
& t=0 \\
& \lambda=\text { the decay constant of the precursor }
\end{aligned}
$$

The probability that a neutron is emitted between $t$ and $t+d t$ is given by

$$
p(t) d t=\lambda e^{-\lambda t} d t
$$

The probability distribution function is given by

$$
\int_{0}^{t} p\left(t^{\prime}\right) d t^{\prime}=1-e^{-\lambda t}
$$


Sampling this distribution results in

$$
t=\frac{\ln (1-r)}{-\lambda} \frac{\ln (r)}{-\lambda}
$$

for the time of emission.

\subsection{Collision or Escape from the Present Region}

After a collision or after belng emitted from the source a neutron is positioned at $(x, y, z)$ and headed in the direction indicated by $(u, v, w)$. The geometry of the problem is divided into cells or regions (see Appendix J) which are bounded by surfaces. The equations of the $N_{i}$ surfaces bounding the $I^{\prime}$ th cell are given by

$$
\begin{gathered}
f_{i, 1}(x, y, z)=0.0 \\
f_{i, 2}(x, y, z)=0.0 \\
\cdot \\
\cdot \\
\cdot \\
f_{i, N_{i}}(x, y, z)=0.0
\end{gathered}
$$

where $N_{1}=$ total number of surfaces bounding the $I^{\prime}$ th cell.

If the neutron lies in the $1^{\prime}$ th cell then the first operation that must be performed is to find the intersections of the neutron's present line of flight [as given by $(u, v, w)$ and $(x, y, z)]$ with the $N_{1}$ surfaces as 
given by Equation A.10. Only positive real intersections are of interest and of these the one that ylelds the shortest distance from the neutron's previous position is used. This distance $d_{\max }$ is the maximum distance the neutron can travel before it leaves the present region.

The probability that a neutron undergoes a collision between $c$ and $c+d c$ is given by

$$
\begin{aligned}
\mathrm{p}(\mathrm{c}) \mathrm{d} c= & { }_{i} \Sigma_{t} \quad e^{-{ }_{i} \Sigma_{t} c} \mathrm{dc} \\
\text { where } i_{t} \Sigma_{t} & \begin{array}{l}
\text { is the total macroscopic cross section of the } \\
\text { material in the } i \text { th cell }
\end{array} \\
& c \text { is the distance as measured from the current } \\
& \text { position }(x, y, z) \text { of the neutron to the point } \\
& \text { of collision }
\end{aligned}
$$

Using equation A.11 the probability distribution function is given by

$$
P(c)=\int_{0}^{c} p\left(c^{\prime}\right) d c^{\prime}=1-e^{-i \Sigma_{t} c}
$$

Sampling this distribution for the distance to the point of collision results in

$$
c=\frac{\ln (r)}{-{ }_{i} \Sigma_{t}}
$$


The decision as to whether the neutron either collides or escapes can then be made as follows:

$$
\text { if } c \leqslant d_{\max }
$$

then the neutron undergoes a collision at

$$
\begin{aligned}
& \left(x^{\prime}, y^{\prime}, z^{\prime}\right) \\
& \text { where } x^{\prime}=x+u c \\
& y^{\prime}=y+v c \\
& z^{\prime}=z+w c \\
& \text { If } c>d_{\text {max }}
\end{aligned}
$$

then the neutron escapes the present cell at the point

$$
\begin{aligned}
& \left(x^{\prime}, y^{\prime}, z^{\prime}\right) \\
& \text { where } x^{\prime}=x+u d_{\text {max }} \\
& y^{\prime}=y+v d_{\max } \\
& z^{\prime}=z+w d_{\max }
\end{aligned}
$$

\section{A.4 Entering a New Region}

When a neutron is crossing an intersection, the time must be updated as given by 


$$
\begin{aligned}
t^{\prime}=t+d_{\max } / \text { Vel } \\
\text { where } t^{\prime}=\text { new time } \\
d_{\max }=\text { distance traveled since } t \text { was calculated } \\
\text { Vel }=\text { velocity of the neutron }=\sqrt{\frac{2 E}{m_{n}}} \\
m_{n}=\text { mass of a neutron. }
\end{aligned}
$$

The decision as to which region is being entered is made by comparing the sense (see Appendix J) of the neutron's present position $\left(x^{\prime}, y^{\prime}, z^{\prime}\right)$ with the senses of points in neighboring cells (the sense with respect to the sirface which the neutron is on is not checked). Since the senses of a cell are unique, only one cell will agree with this sense check. This cell and its material are used as the next cell.

\section{A.5 Collision Type}

After it has been determined that a collision has occurred (see Appendix A.3) the time of the particle must be updated as given by equation A.1 except that c (see equation A.13) is used instead of $d_{\max }$. The following decisions must be made:

(1) which nuclide is the neutron interacting with (for materials composed of more than one nuclide)

(2) what type of reaction occurs. 
If the total cross section of nuclide $j$ of the $1^{\prime}$ th cell is given by $i, j{ }_{t}$, then the probability that the neutron interacts with nuclide $j$ is given by

$$
p_{j}=\frac{i, j^{\Sigma_{t}}}{\Sigma}, \quad j=1, \ldots, M_{i}
$$

where $i^{\Sigma_{t}}$ is defined by equation $A .11$ and $M_{1}$ is the number of nuclides making up the material in cell $i$. Adding the probabilities given by $p_{j}$ results in the probability distribution function

$$
P_{j}=\sum_{i=1}^{j} n_{1}
$$

The $P_{f}$ is sampled by selecting a random number $r$ and

$$
\begin{aligned}
& \text { If } P_{k}<r<P_{k+1} \quad k=0, \ldots, M_{i}-1 \\
& \text { where } P_{0}=0.0 \\
& \text { then the neutron interacts with the }(k+1) \text { nuclide. }
\end{aligned}
$$

The probability of reaction 1 occurring is given by

$$
\begin{aligned}
& P_{1}=\frac{\sigma_{f, 1}}{\sigma_{j, t}} \quad 1=1, L_{j} \\
& \text { where } L_{j}=\text { the number of possible reactions for the } f \text { 'th } \\
& \text { isotope }
\end{aligned}
$$




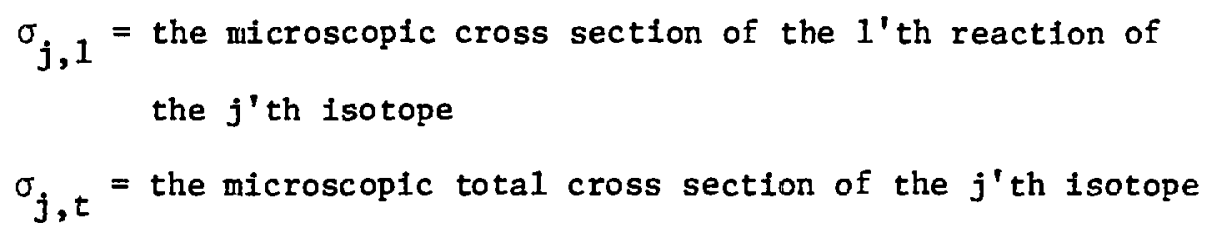

The resulting probability distribution is given by

$$
\mathrm{p}_{1}=\sum_{1=1}^{L_{j}} \mathrm{p}_{1}
$$

and is sampled by the random number $r$ where

$$
\begin{aligned}
& \text { if } P_{k}<r<P_{k+1} \quad k=0, \ldots, M_{1}-1 \\
& \text { where } P_{0}=0.0 \\
& \text { then the neutron undergoes the }(k+1) \text { 'th reaction }
\end{aligned}
$$

\section{A.6 Scattering}

In this section both elastic and inelastic scattering events are described. A scattering event results in a new set of $(u, v, w)$ and a new E. These new coordinates are dependent only upon the scattering angle of the neutron in the laboratory system, (see Figure A.14) and the original $(u, v, w)$. Because scattering is often isotropic in the center of mass (COM) coordinates, the scattering angle in the COM system, $\theta$, is usually used instead of $\phi$. 


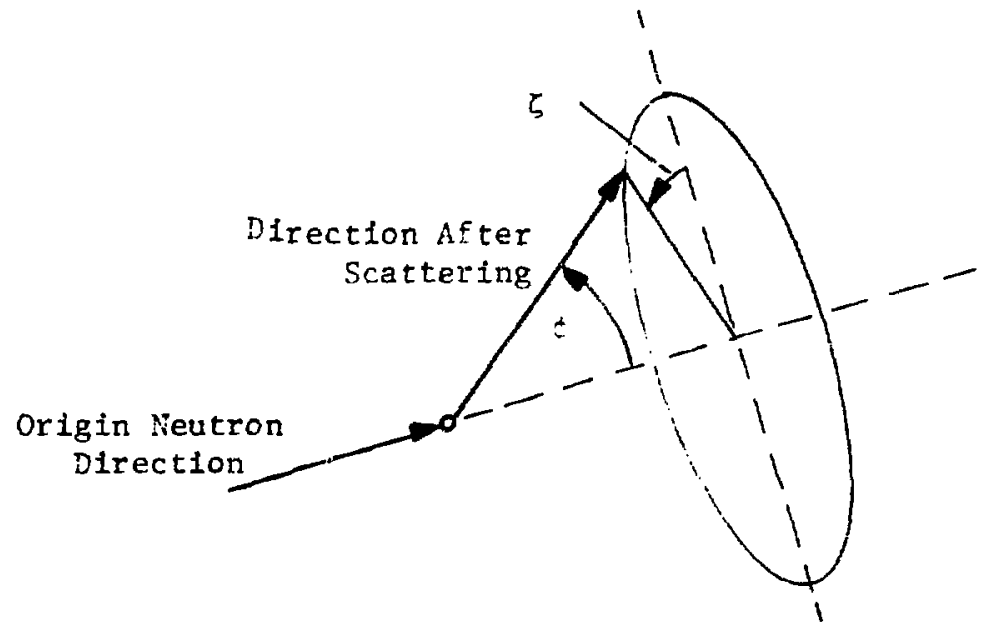

Figure $A .14$ Scattering in the Lab System

\section{A.6.1 Elastic Scattering}

For isotropic scattering in the COM system the scattering angle $\theta$ can be sampled by

$$
\cos \theta=2 r-1
$$

which when converted to the Iaboratory system results in

$\cos \phi=\frac{1+A \cos \theta}{\sqrt{A^{2}+2 A \cos \theta+1}}$

where $A$ is the mass of the target nucleus in units of the mass of a neutron 
The angle $\zeta$ (see Figure A.14) is sampled by

$$
\zeta=2 \pi r
$$

The above $\zeta$ and $\cos \phi$ can then be converted to a new $\left(u^{\prime}, v^{\prime}, w^{\prime}\right)$ by using suitable transformations and the $(u, v, w)$ before the collision.

The emerging neutron energy in the lab system is given by

$$
\begin{aligned}
& E^{\prime}=\frac{E}{2}\{(1-\alpha) \cos \theta+1 \div \alpha\} \\
& \text { where } \alpha=\left(\frac{A-1}{A+1}\right)^{2} \\
& E=\text { incoming neutron energy in the lab system }
\end{aligned}
$$

In the event that scattering is not isotropic in the COM system (i.e. $\left.\sigma_{s}(\theta) \neq \sigma_{s} / 4 \pi\right)$ tables of $\sigma_{s}(\theta)$ vs. $\theta$ are usually supplied and can be used in the same manner as the source energy tables (see section A.2.3). Once a value of $\cos \theta$ has been sampled the process is the same as for isotropic scattering.

The above descriptions have assumed that the bombarded nucleus is stationary in the lab system. If this is not true, a thermal scattering treatment rust be used as is described in reference 9. 


\section{A.6.2 Inelastic Scattering}

The sampling of $\cos \theta$ and $\zeta$ is the same for Inelastic scattering as it is for elastic scattering; however, since the nucleus is excited, equations A.23 and A.25 are no longer val1d. The energy of the emerging neutron in the COM system after an $\left(n, n^{\prime}\right)$ reaction is given by

$$
E_{\operatorname{COM}}^{\prime}=\left(\frac{A}{A+1}\right)^{2} E+Q\left(\frac{A}{A+1}\right)
$$

where $E$ is the enorgy of the incident neutron in the lab system and $Q$ Is the $Q$ value of the reaction $(Q=$ rest energy of the nucleus before collision - rest energy after). The value of $Q$ is determined by the particular $\left(n, n^{\prime}\right)$ reactions; however, for heavy nuclei the levels of $\left(n, n^{\prime}\right)$ reactions can become very dense. As a result $Q$ may in some cases have to be sampled. Once $E_{C O M}^{\prime}$ has been determined, the emergent energy in the lab system can be found by the relation

$$
E_{L a b}^{\prime}=E_{C O M}^{\prime}+\frac{\left[E+2 \cos \theta(A+1) \sqrt{E E_{C O M}^{\prime}}\right]}{(A+1)^{2}}
$$

and the scattering angle in the lab system is given by

$$
\cos \phi=\sqrt{\frac{E_{C O M}^{\prime}}{E^{\prime}}} \cos \theta+\sqrt{\frac{E}{E^{\prime}}}\left(\frac{1}{A+1}\right)
$$


After determining $\phi$ and $\zeta$ the new $(u, v, w)$ is iound In the same way as for the elastic scattering case.

\section{A.7 Other Reactions}

Other reactions that can occur besides scattering events are capture, fission, $(n, 2 n)$, etc. The capture event is treated differently than any other reaction and involves the concept of the neutron "weight". The weigist of a neutron can be thought of as representing a fraction of a neutron. Although only an integer number of neutrons can be transported, a fraction of a neutron can be represented by a neutron with a welght less than one (weights are further discussed in section 2.2). Whenever a neutron uniergoes a collision with a nucleus the weight of the neutron is multiplied by

$$
\frac{\sigma_{t}-\sigma_{c}}{\sigma_{t}}
$$

where $\sigma_{c}$ is the capture cross section. The reason for this treatment of capture will be given in section 2.2 .

of the other possible reactions only fission will be described In this section. Three features must be sampled for the neutrons emitted from fission:

(1) the number of neutrons emitted 
(2) the energy of the neutrons emitted

(3) the directions of neutrons emitted.

The neutrons are emitted 1sotropically in the lab system resulting in the same sampling schene shown in Figure A.12 for the new $(u, v, w)$.

The average number of neutrons emitted per fission for an Incoming energy $E$ is given by ${ }^{18}$

$$
\bar{v}(E)=v_{0}+a E
$$

where $V_{0}$ and a are experimentally determined constants which depend on the fissionsing nucleus. It is possible to sample the distributions for each reaction based on $\sigma_{f}(n, n), \sigma_{f}(n, 2 n), \sigma_{f}(n, 3 n)$, etc. and follow the corresponding number of neutrons. However, it is unnecessary since a single neutron can be created with a welght equal to $V(F)$.

The energy distribution of neutrons emitted from fission can be approximated by ${ }^{10}$

$X\left(E^{\prime}\right) d E^{\prime}=.453 e^{-1.036 E^{\prime}} \sinh \sqrt{2.29 E^{\prime}}$

where $X\left(E^{\prime}\right) \mathrm{dE}^{\prime}$ is the probability that a fission neutron is emitted with energy between $E$ and $E^{\prime}+d^{\prime}$ (lab system) 
The value of $E$ is found by sampling the probability distribution function

$$
r=\int_{0}^{E} X\left(E^{\prime}\right) d E^{\prime} .
$$

The method for performing this sampling is non-trivia? and is given in references 41 and 42 .

\section{A.8 Tallies}

A Monte Carlo tally can be anything the user wishes to specify. Some commonly used tallies are:

(1) the current of neutrons crossing a specified surface

(2) the number of neutron collisions in a cell

(3) the number of fissions in a cell

(4) the flux of neutrons at a point.

In most cases, the neutron can contribute to the tally more than once during its lifetime. If each contribution to the tally if the $n$ 'th neutron is given by $x_{1, n}$ then the total contribution of the n'th neutron is given by

$$
x_{n}=\sum_{i=1}^{c} x_{i, n}
$$


where $C_{n}$ is the number of times the $n^{\prime}$ th neutron contributes to the tally. It is the value of $x_{n}$ that is used to calculated the mean and variance of the tally.

The tally $x_{n}$ is evaluated when the neutron has escaped the system (see Figure A.2). The system is defined as that region of state space which is of interest to the tally or tallies under consideration. For example, consider a single tally that consists of counting the number of neutrons with energies between $E_{1}$ and $E_{2}$ $\left(E_{2}>E_{1}\right)$ crossing a surface. For this example, whenever a neutron's energy falls below $E_{1}$, the neutron can no longer contribute to the tally and therefore has lefe the system. Similar situations exist. for the other state space variables.

The individual contributions $x_{1, n}$ must be saved whenever they occur. For the example tallies presented earlier, this is done

(1) When a neutron crosses a surface bounding two ceils, this surface is checked. If the surface corresponds to a tally surface, $x_{i, n}$ is calculated.

(2) When a new cell is entered, a check is male to see whether or not the cell is a tally cell. -f it 18, then $x_{i, n}$ is calculated each time a collision occurs in the cell.

(3) Same as (2) except for fissions. 
(4) After being emitted from the source and after each collision, the probability of being scattered toward the unit area of the point detector is calculated. This probability is multiplied by the probability of the neutron arriving at the point detector uncollided and weight of the neutron prior to the collision resulting in $x_{i, n^{*}}$ (The point detector is described further in section 5.1.3). 


\section{Eppendix B. Statistical Errors in Monte Carlo}

Section B.I derives the expression for the variance of the sample mean. The central. limit theorem is discussed in secticn B.2.

\section{B.I Derivation of the Sample Mean}

Let $x(s)$ be any integrable function of $s$ and $p(s)$ be the probability density of $s$. The mean or expected value of $x$ is given by

$$
\langle x\rangle=\int_{-\infty}^{\infty} x(s) p(s) d s
$$

The variance of $x$ is given by

$$
\begin{aligned}
\sigma^{2}(x) & =\int_{-\infty}^{\infty}(x(s)-\langle x\rangle)^{2} p(s) d s \\
& =\int_{-\infty}^{\infty} x^{2}(s) p(s) d s-2\langle x\rangle \int_{-\infty}^{\infty} x(s) p(s) d s+\langle x\rangle^{2} \int_{-\infty}^{\infty} p(s) d s \\
& =\left\langle x^{2}\right\rangle-2\langle x\rangle^{2}+\langle x\rangle^{2} \\
& =\left\langle x^{2}\right\rangle-\langle x\rangle^{2}
\end{aligned}
$$


When $\mathrm{N}$ values of the random variable $s, s_{1}, s_{2}, \ldots \ldots, s_{\mathrm{N}}$,

are choosen according to the frobability density $p(s)$ the resulting values of $x\left(s_{i}\right)$ are given by $\left.x\left(s_{1}\right), x\left(s_{2}\right), \ldots \ldots \ldots, s_{N}\right)$. The sample mean is given by

$$
\bar{x}=\frac{\sum_{i=1}^{N} x\left(s_{i}\right)}{i}
$$

The expected value of the sample mean is given by

$$
\begin{aligned}
\langle\bar{x}\rangle & =\left\langle\frac{\sum_{i=1}^{N} x\left(s_{i}\right)}{N}\right\rangle \\
& =\frac{1}{N} \int_{-\infty}^{\infty} \int_{-\infty}^{\infty} \ldots \ldots \int_{-\infty}^{\infty}\left[\sum_{i=1}^{N} x\left(s_{i}\right)\right] p\left(s_{1}\right) \ldots p\left(s_{N}\right) d s_{1} \ldots d s_{N}
\end{aligned}
$$

where each $s_{i}$ has been treated as an independent varlable. ${ }^{4}$ Esing

$$
\int_{-\infty}^{\infty} P\left(s_{i}\right) d s_{i}=1
$$

Equation B. 4 reduces to

$$
\begin{aligned}
\langle\bar{x}\rangle & =\frac{1}{N} \int_{-\infty}^{\infty} \sum_{i=1}^{N} x\left(s_{i}\right) p\left(s_{i}\right) d s_{i} \\
& =\frac{1}{N} \sum_{i=1}^{N} \int_{-\infty}^{\infty} x\left(s_{i}\right) p\left(s_{i}\right) d s_{1}
\end{aligned}
$$




$$
\langle\bar{x}\rangle=\frac{1}{\pi} \sum_{i=1}^{n}\left\langle x\left(s_{1}\right)\right\rangle=\langle x\rangle
$$

Using Equation 3.2, the variance of the sample nean about $\langle x\rangle$ is given by

$$
\sigma^{2}(\bar{x})=\left\langle\bar{x}^{2}\right\rangle-\langle x\rangle^{2}
$$

where the first tern is given by

$$
\left.\bar{x}^{2}\right\rangle=\frac{1}{N^{2}}\left\langle\left[\sum_{i=1}^{N} x\left(s_{i}\right)\right]^{2}\right\rangle
$$

$$
\begin{aligned}
& =\frac{i}{N^{2}} \int_{-\infty}^{\infty} \int_{-\infty}^{\infty} \ldots \int_{-\infty}^{\infty}\left[\sum_{i=1}^{x} x\left(s_{i}\right)\right]^{2} p\left(s_{1}\right) \ldots p\left(s_{N}\right) d s_{1} \ldots d s_{N} \\
& =\frac{1}{N^{2}} \int_{-\infty}^{\infty} \int_{-\infty}^{\infty} \ldots \int_{-\infty}^{\infty}\left[\sum_{i=1}^{x} x^{2}\left(s_{i}\right) \div \sum_{i=1}^{x} \sum_{\substack{j=1 \\
j \neq i}}^{x} x\left(s_{i}\right) x\left(s_{j}\right)\right] p\left(s_{1}\right) \ldots p\left(s_{N}\right) d s_{1} \ldots d s_{N}
\end{aligned}
$$


Using Equation B.5, Equation B.8 reduces to

$$
\begin{aligned}
\left.\vec{x}^{2}\right\rangle & =\frac{1}{N^{2}}\left[\int_{-\infty}^{\infty} \sum_{i=1}^{N} x^{2}\left(s_{i}\right) p\left(s_{i}\right) d s_{i}+\int_{-\infty}^{\infty} \sum_{i=1}^{N} x\left(s_{i}\right) p\left(s_{i}\right) d s_{i} \int_{-\infty}^{\infty} \sum_{\substack{j=1 \\
j \neq i}}^{N} x_{j}\left(s_{j}\right) p\left(s_{j}\right) d s_{j}\right] \\
& \left.=\frac{1}{N^{2}}\left[\sum_{i=1}^{N} x^{2}\left(s_{i}\right)\right\rangle+\sum_{i=1}^{N} \sum_{\substack{j \neq 1 \\
j \neq i}}^{N}\left\langle x\left(s_{i}\right)\right\rangle^{2}\right] \\
& =\frac{\langle x}{N}+\frac{(N 1)<x\rangle^{2}}{N}
\end{aligned}
$$

Substituting Equation B.9 Into Equation B.7 results in

$$
\sigma^{2}(\bar{x})=\frac{\left\langle x^{2}\right\rangle}{N}+\frac{(N-1)}{N}\langle x\rangle^{2}-\langle x\rangle^{2}=\frac{\left\langle x^{2}\right\rangle-\langle x\rangle^{2}}{N}=\frac{\sigma^{2}(x)}{N} \text { (B.10) }
$$

which is the desired result. 

Substituting

$$
t=\frac{\bar{x}-\langle x\rangle}{\sigma(x)}
$$

and

$$
d t=\frac{d \bar{x}}{\sigma(\bar{x})}
$$

into Equation B.12 gives

$\operatorname{prob}[\alpha \sigma(\bar{x})<\bar{x}-\langle x\rangle<\beta \sigma(\bar{x})]=\frac{1}{\sqrt{2 \pi}} \exp \left(-t^{2} / 2\right) d t$

which is the desired result. 


\section{Appendix C: FORTRAN Coding for the One Dimensional Homogeneous Slab Monte Carlo Problem}

The following computer listing has had all input - output and other extraneous coding removed. The significant FORTRAN variables are defined below.

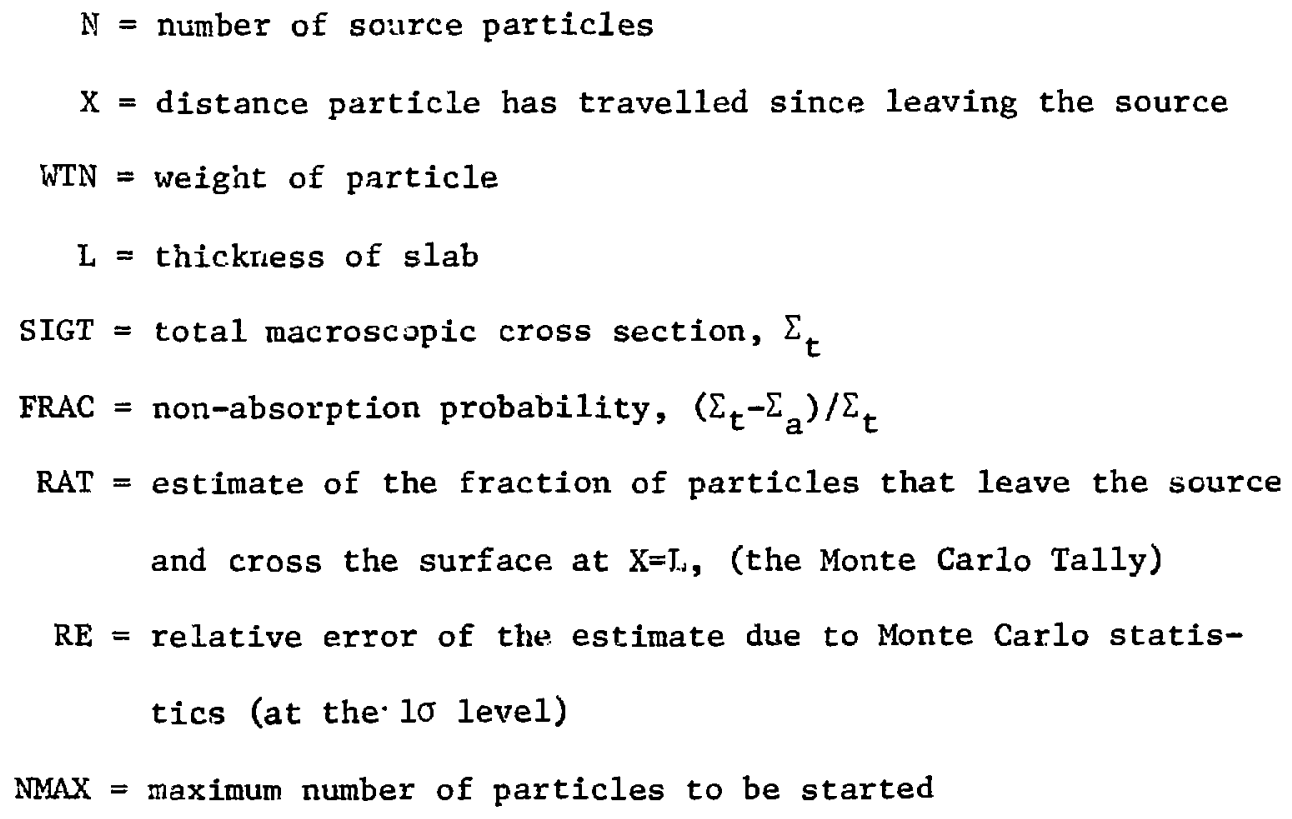




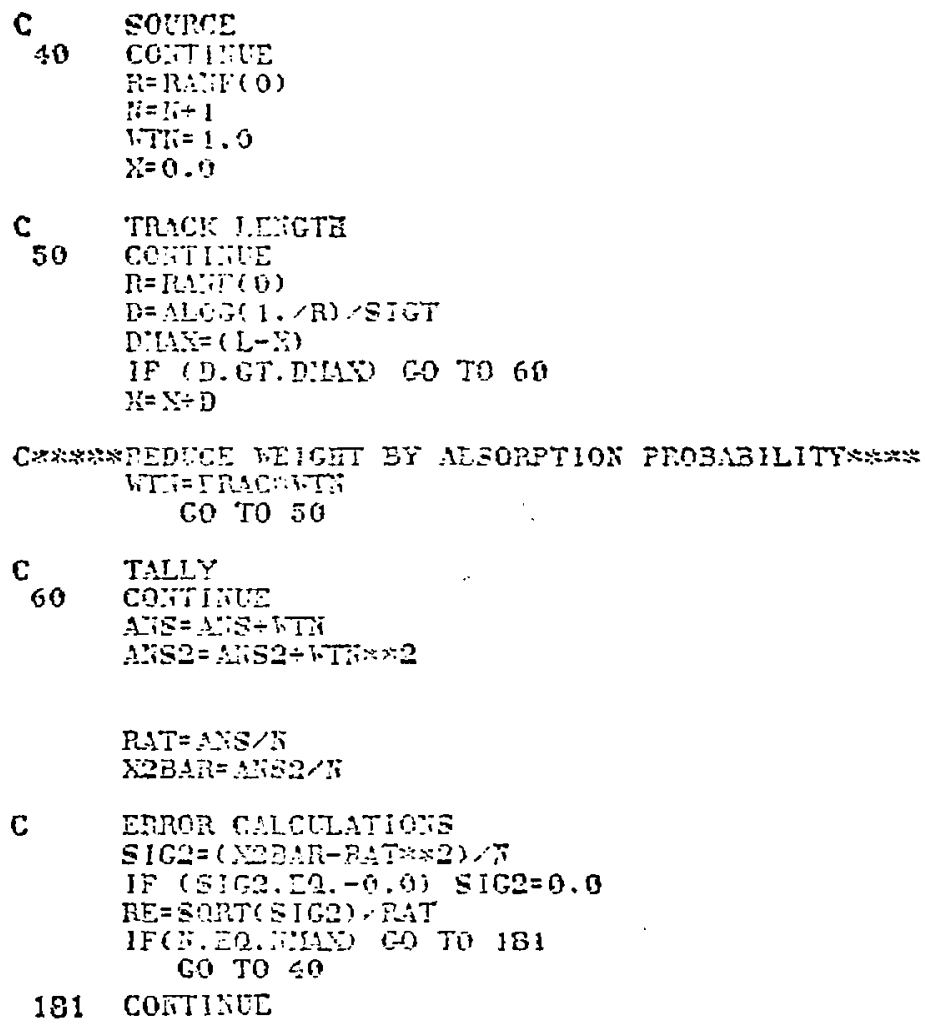

181 COHTIXEL 
Appendix D: FOR'RAN Coding for the Fractional Correction Rule Deterministic Classifier

The following computer listing has had all input - output and other extranecus coding removed. The term "TEACHER" in the comment statements refers to the classification that a track appears to belong to after observing the importances created by the last source particle. The term "STUDENT" refurs to the classification given by the current discriminant function. The Monte Carlo parameters are the same as those described in Appendix C. Significant pattern recognition parameters are given below.

INC $=$ the number of tracks created by the last source particle (also used to number the prototypes)

$$
\text { WTREM }(I)=\text { the weigh }=\text { of the particle after the I'th collision }
$$
(the source is the first collision) $\operatorname{NXREM}(I)=$ the distance from the source to the $I^{\prime}$ th collision (source is the first collision). These values are used as the prototypes for the pattern classifier.

$G=$ the diecriminant function, $g(Y)$

$W T(I)=$ the I'th component of the weight vector, $W=w_{i}$ SEP2 = upper limit of the importance buffer zone used to separate classes, $\mathrm{I}_{2}$

SEP1 = lower limit of the importance buffer zone used to separate classes, $I_{1}$

SUBTOT $(I)=$ number of prototypes belonging to the $I^{\prime}$ th class (as seen by the "TEACHER") 


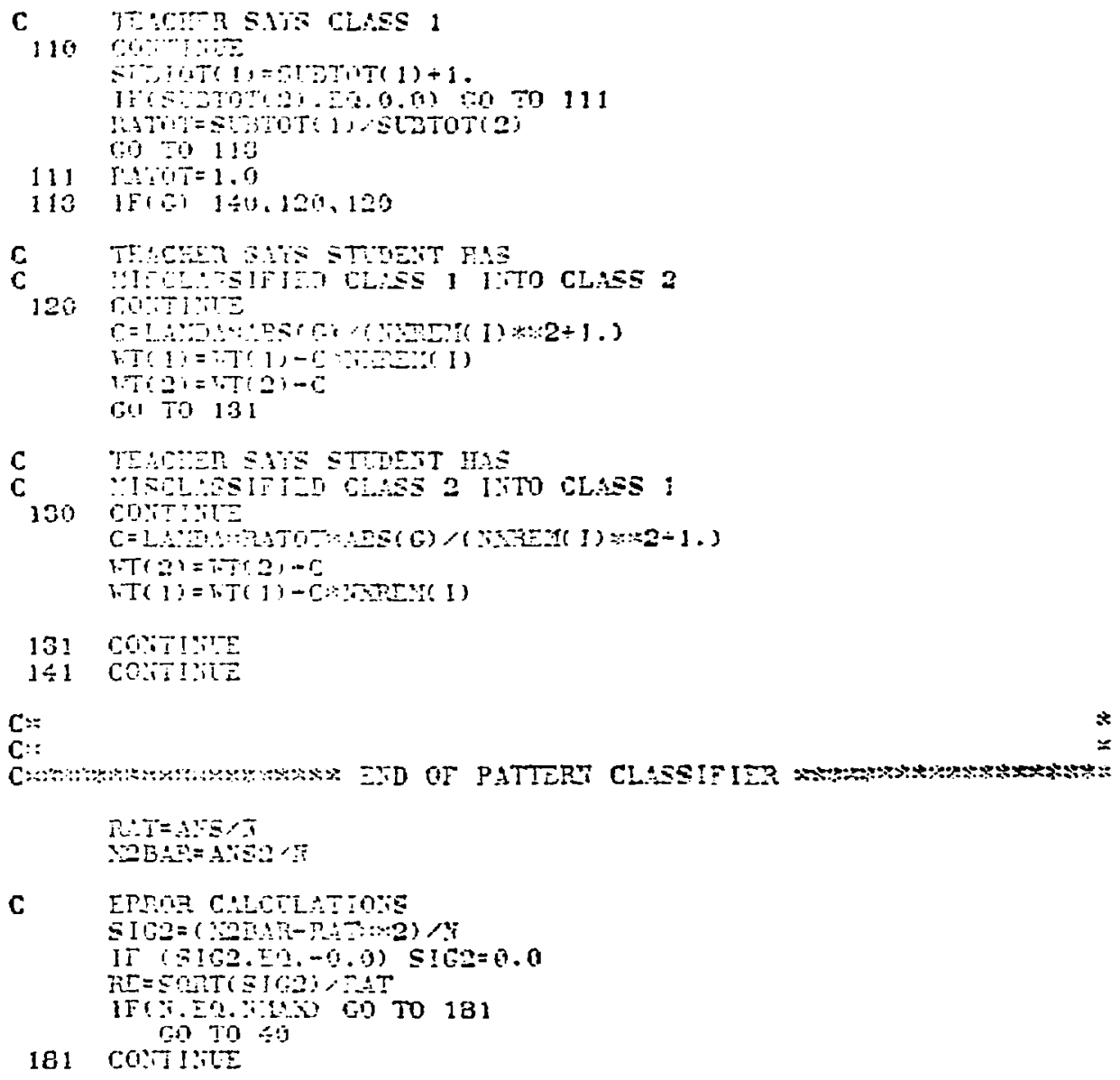




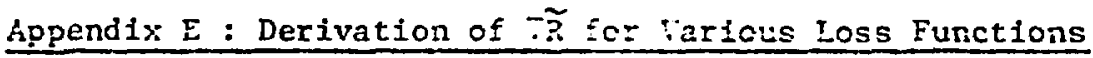

An approximation to the Iisk has given by Equation 3.15 and is $r \in$ peated here as

$\tilde{R}(i)=\sum_{k=1}^{2} \frac{1}{N_{k}} \sum_{n=1}^{N_{k}} s\left(i, v_{n}^{k}, c_{i}^{\prime} c_{i}\right) \quad i=1,2 \quad$ ifk

where:

$$
\begin{aligned}
& M_{k}=\text { rumber } 0 \text { prototipes in class } c_{k} \\
& \mathrm{Y}_{\mathrm{n}}^{\mathrm{k}}=\mathrm{n}^{\prime} \text { th aisclassisied prototype of class } C_{k} \\
& S\left(W, Y_{n}^{k}, C_{i} \mid C_{k}\right)=10 \text { s incurred inen prototype } Y_{n}^{k} \text { actually } \\
& \text { belonging to class } c_{k} \text { is placed in class } C_{i}
\end{aligned}
$$

The gradient of $R(P)$ for one $\dot{c} i=e: s i c r a l$ feature space is given by

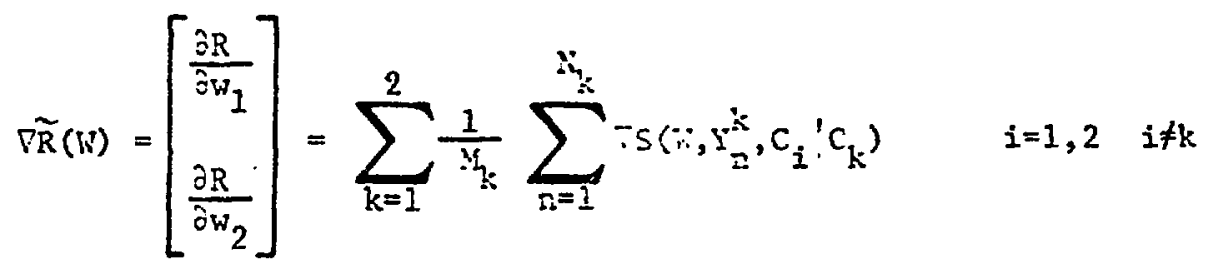

$$
=\frac{1}{N_{1}} \sum_{n=1}^{N_{1}} \nabla s\left(n, r_{n}^{1}, c_{2}\left(c_{1}\right)+\frac{1}{x_{2}} \sum_{n=2}^{n_{2}} r s\left(n, r_{n}^{2}, c_{1} ! c_{2}\right)\right.
$$




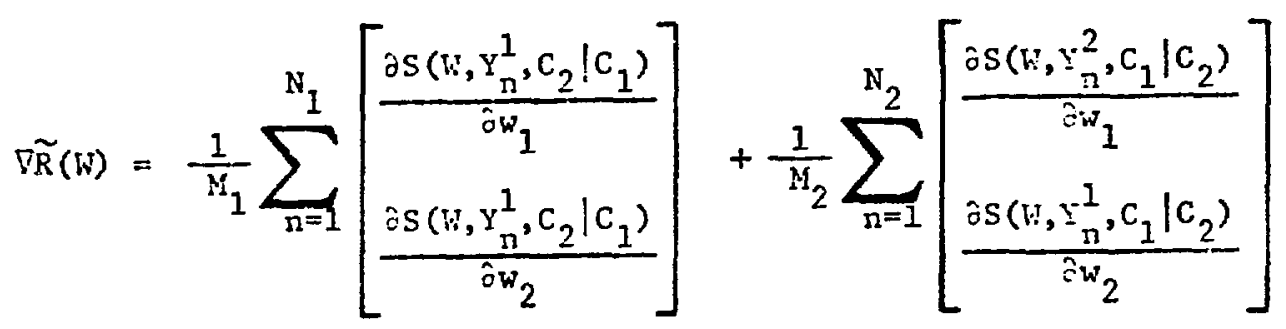

Equation E.2 will now be evaluated for several different loss functions. Loss Function $S=d$

The distance $d$ fron a prototype, $Y$, to the decision surface $g(Y)$ $=0$ in feature space is given by

$$
d=\frac{|w \cdot Y *|}{\left|W^{\prime}\right|}
$$

where $W^{\prime}$ is the weight vector $W$ with $w_{N+1}=0$ and $Y^{*}$ is the augumented feature vector as given by Equation 3.5. For $S=d$, the gradient of $S$ for prototypes belonging to class $C_{1}$ but misclassified into $C_{2}$ is given by

$$
\nabla S\left(w, Y_{n}^{1}, c_{2} \mid c_{1}\right)=\nabla S\left(d, c_{2} \mid c_{1}\right)=\frac{\partial S}{\partial d}\left[\begin{array}{l}
\frac{\partial d}{\partial w_{1}} \\
\frac{\partial d}{\partial w_{2}}
\end{array}\right]
$$


Substituting Equation E.3 into Equation E.: and using the relation

$$
\frac{\partial S}{\partial d}=1
$$

results in

$$
\nabla S\left(w, Y_{n}^{1}, c_{2} \mid c_{1}\right)=\left[\begin{array}{l}
\frac{\partial d}{\partial w_{1}} \\
\frac{\partial d}{\partial w_{2}}
\end{array}\right]=\left[\begin{array}{c}
\frac{\partial\left(\frac{w_{1} y+w_{2}}{w_{1}}\right)}{\partial w_{1}} \\
\frac{\partial\left(\frac{w_{1} y+w_{2}}{w_{1}}\right)}{\partial w_{2}}
\end{array}\right]=\left[\begin{array}{c}
-\frac{w_{2}}{w_{1}^{2}} \\
\frac{1}{w_{1}}
\end{array}\right]
$$

where $y$ is the single component of the feature vector $Y_{n}^{1}$ (Equation E.1) and $w_{1}$ and $w_{2}$ are the components of the weight vector $W$ which exist at the time prototype $\mathrm{Y}_{\mathfrak{n}}^{1}$ is misclassified. Similarly for prototypes belonging to class $C_{2}$ but misclassified into class $C_{1}$, the gradient of $\mathrm{S}$ is given by

$$
\nabla s\left(W, Y_{n}^{2}, c_{1} \mid C_{2}\right)=\left[\begin{array}{c}
\frac{w_{2}}{w_{1}} \\
-\frac{1}{w_{1}}
\end{array}\right]
$$




\section{Loss Eunction $S=\sqrt{2}$}

For this loss function

$$
\frac{\partial S}{\partial \dot{d}}=\frac{\partial(\cdot \bar{d})}{\partial d}=\frac{1}{2 \sqrt{d}}
$$

For Eçuation E.3 and $\xi=n \cdot Y$, Eçution E. 8 reduces to

$$
\frac{\partial s}{\partial d}=\frac{1}{2}\left(\left|\frac{w_{1}}{g}\right|\right)^{1 / 2}
$$

For Ecuations E.9 and E.6, Eçizsion E.4 reduces to

$$
\nabla s\left(1, v_{n}^{1}, c_{2} \mid c_{1}\right)=\frac{1}{2}\left(\left|\frac{w_{1}}{g}\right|\right)^{1 / 2}\left[\begin{array}{c}
-\frac{w_{2}}{2} \\
w_{1} \\
\frac{1}{w_{1}}
\end{array}\right]
$$

Sirlarly, for prototypes belonging to class $C_{2}$ but misclassified in:o class $C_{1}$, the gradient of $S$ is given by 


$$
\nabla S\left(u_{1}, v_{n}^{2}, c_{1} \mid c_{2}\right)=\frac{1}{2}\left(\left|\frac{w_{1}}{g}\right|\right)^{1 / 2}\left[\begin{array}{c}
\frac{w_{2}}{w_{1}^{2}} \\
-\frac{1}{w_{1}}
\end{array}\right]
$$

Loss Function $S=d^{2}$

This loss function results in

$$
\frac{\partial S}{\partial d}=\frac{\partial\left(d^{2}\right)}{\partial d}=2 d=2\left|\frac{g}{w_{1}}\right|
$$

winich when substituted Into Equation E.4 along with Equation E.6 results in

$$
\nabla s\left(w, v_{n}^{1}, c_{2} \mid c_{1}\right)=2\left|\frac{g}{w_{1}}\right|\left[\begin{array}{c}
-\frac{w_{2}}{w_{1}} \\
\frac{1}{w_{1}}
\end{array}\right]
$$


and sirflarly for class $C_{2}$ prototypes

$$
\nabla s\left(w_{1} Y_{n}^{2} c_{1} ! c_{2}\right)=2\left|\frac{g}{w_{1}}\right|\left[\begin{array}{c}
\frac{w_{2}}{w_{1}^{2}} \\
-\frac{1}{w_{1}}
\end{array}\right]
$$

Loss Eunction $S=0$

The distance in weight space from the weight vector $W$ to the pattera hyperplane defined by $Y$ is given by

$$
D=\frac{|W \cdot \because *|}{\left|Y * P^{*}\right|}
$$

This is the same distance that is used by the deterministic fractional correction rule as described in section 3.2 .1 and isplemented in Section 4.2.1. lising Equations E.3 and E.15 the relationship betrieen $D$ and $d$ is given by

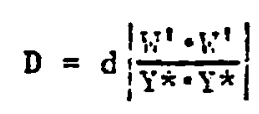

which for one dimensional feature space reduces to 


$$
D=d \frac{\left|w_{1}\right|}{\sqrt{y_{1}^{2}+1}}
$$

For $y_{I}>1$ (which is usually the case for the unnormalized runs made in this research)

$$
D \simeq d\left|\frac{w_{1}}{y_{1}}\right|
$$

Equation E.18 indicates that for $\mathrm{y}_{1} \gg 1$, when using loss $=D$, prototypes with small $y_{1}$ will have more effect on the classifier than those with large $y_{1}$.

For one dimensional feature space and $S=D$

$$
\left[\begin{array}{c}
\frac{\partial D}{\partial w_{1}} \\
\frac{\partial D}{\partial w_{2}}
\end{array}\right]=\frac{1}{\sqrt{y_{1}^{2}+1}}\left[\begin{array}{c}
\frac{\partial\left(w_{1} y_{1}+w_{2}\right)}{\partial w_{1}} \\
\frac{\partial\left(w_{1} y_{1}+w_{2}\right)}{\partial w_{2}}
\end{array}\right]=\frac{1}{\sqrt{y_{1}^{2}+1}}\left[\begin{array}{l}
y_{1} \\
1
\end{array}\right]
$$

and since

$$
\frac{\partial S}{\partial D}=1
$$


the resulting expression for the gradient of $S$ is given by

$$
\nabla S\left(l, y_{n}^{1}, c_{2} \mid c_{1}\right)=\frac{1}{\sqrt{y_{1}^{2}+1}}\left[\begin{array}{c}
y_{1} \\
1
\end{array}\right]
$$

and similarly

$$
\operatorname{PS}\left(W, \mathrm{r}_{\mathrm{n}}^{2}, \mathrm{C}_{1} \mid \mathrm{C}_{2}\right)=\frac{1}{\sqrt{y_{1}^{2}+1}}\left[\begin{array}{c}
-y_{1} \\
-1
\end{array}\right]
$$


Appendix F: FORTRAN Coding for the Statlstical Pattern Classifier using Loss $=\mathrm{d}$

The following computer listing has had all input - output a ? other extraneous coding removed. The parameters defined in Appendices $C$ and $D$ are the same here with the below additional parameter.

$$
\begin{gathered}
\operatorname{GrRISK}(I)=\frac{\frac{\partial R}{\partial w_{I}}}{I=1,2} \quad \text { (see Equation 4.6) }
\end{gathered}
$$

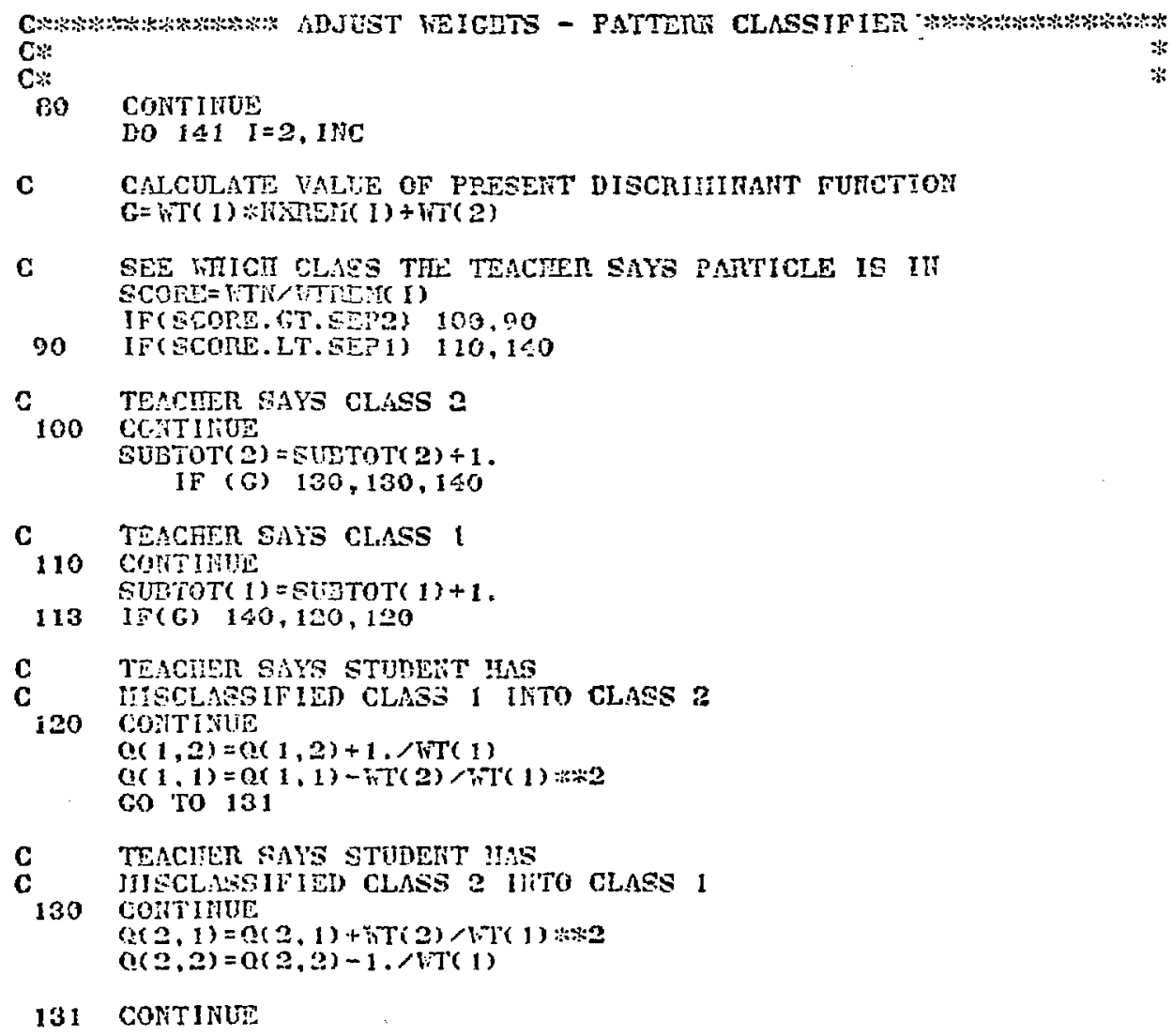

131 CONTINUT 


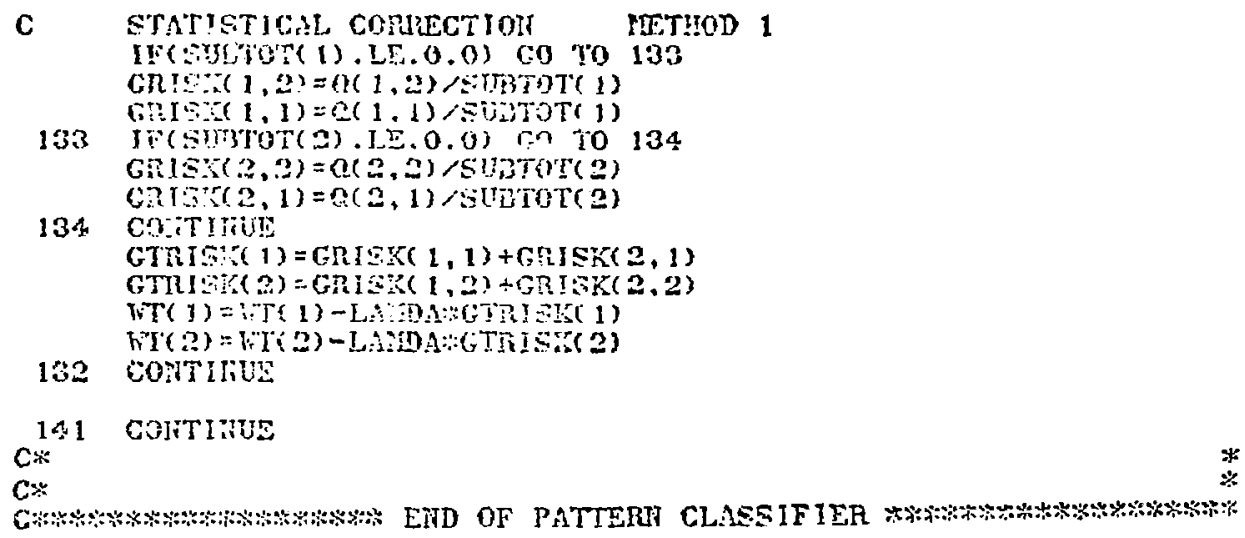


Appendix G: FORTRAN Coding For the One Dimensional Multi-Region Slab

The following computer listing has had all input - output and other extraneous coding removed. The parameter IA denotes the region that the particle is in. The parameters FRAC(I) and SIGT(I) have the same meaning as in Appendix $\mathrm{C}$ only with respect to the $I^{\mathbf{2}}$ th region. Additional parameters are listed below.

\footnotetext{
$\mathrm{NIA}=$ number of regions, also the number of the region containing the tallying surface

$\mathrm{TL}(I)=$ the boundary of the $I^{\prime}$ th region $\mathrm{TL}(I)>\mathrm{TL}(I-I)$
} 


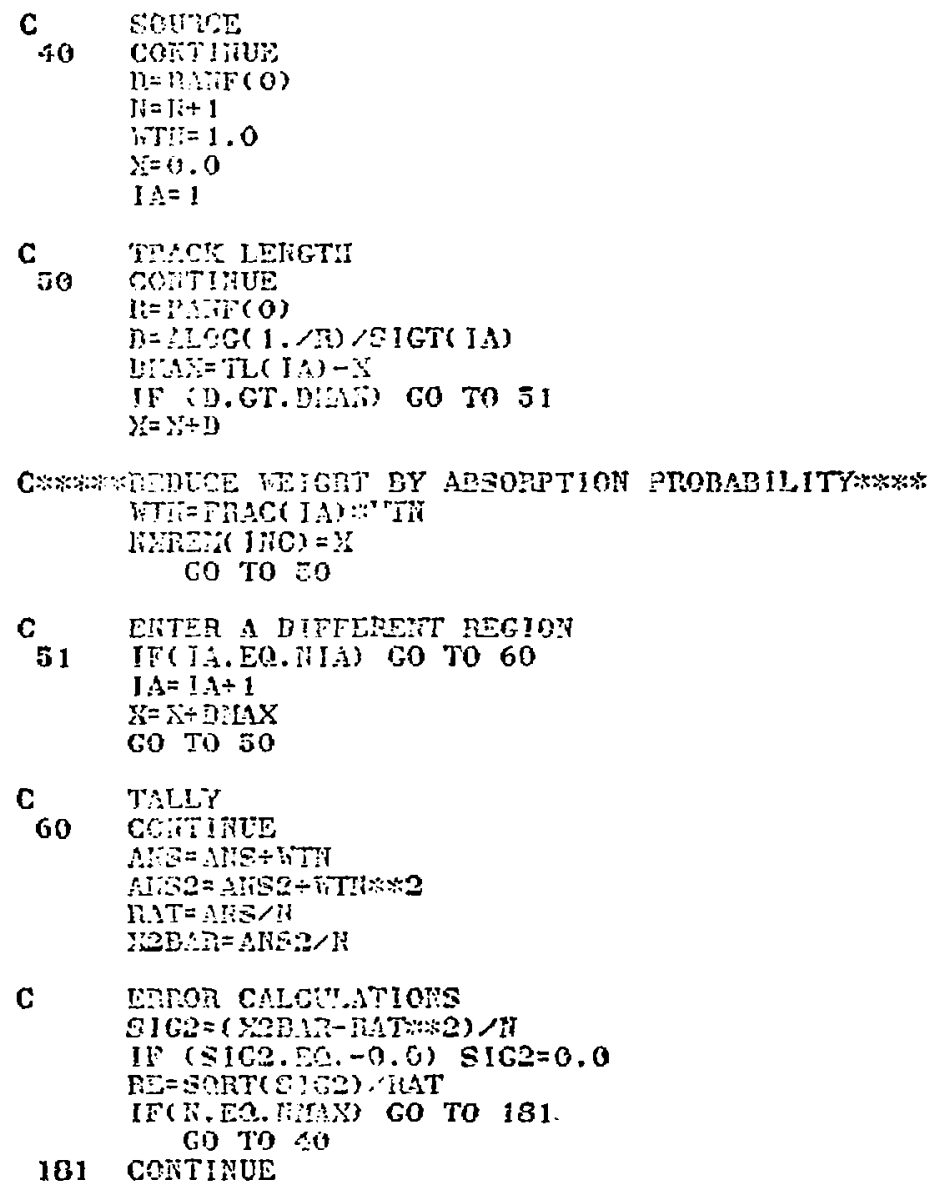


Appendix H: FORTRAN Coding for the Two Dimensional Multi-Region Slab with a Deterministic Pattern Classifier

The followirg computer listing has had all input - output and other extraneous coding removed. The parameters required in addition to those described in Appendices $G, D$, and $C$ are listed below.

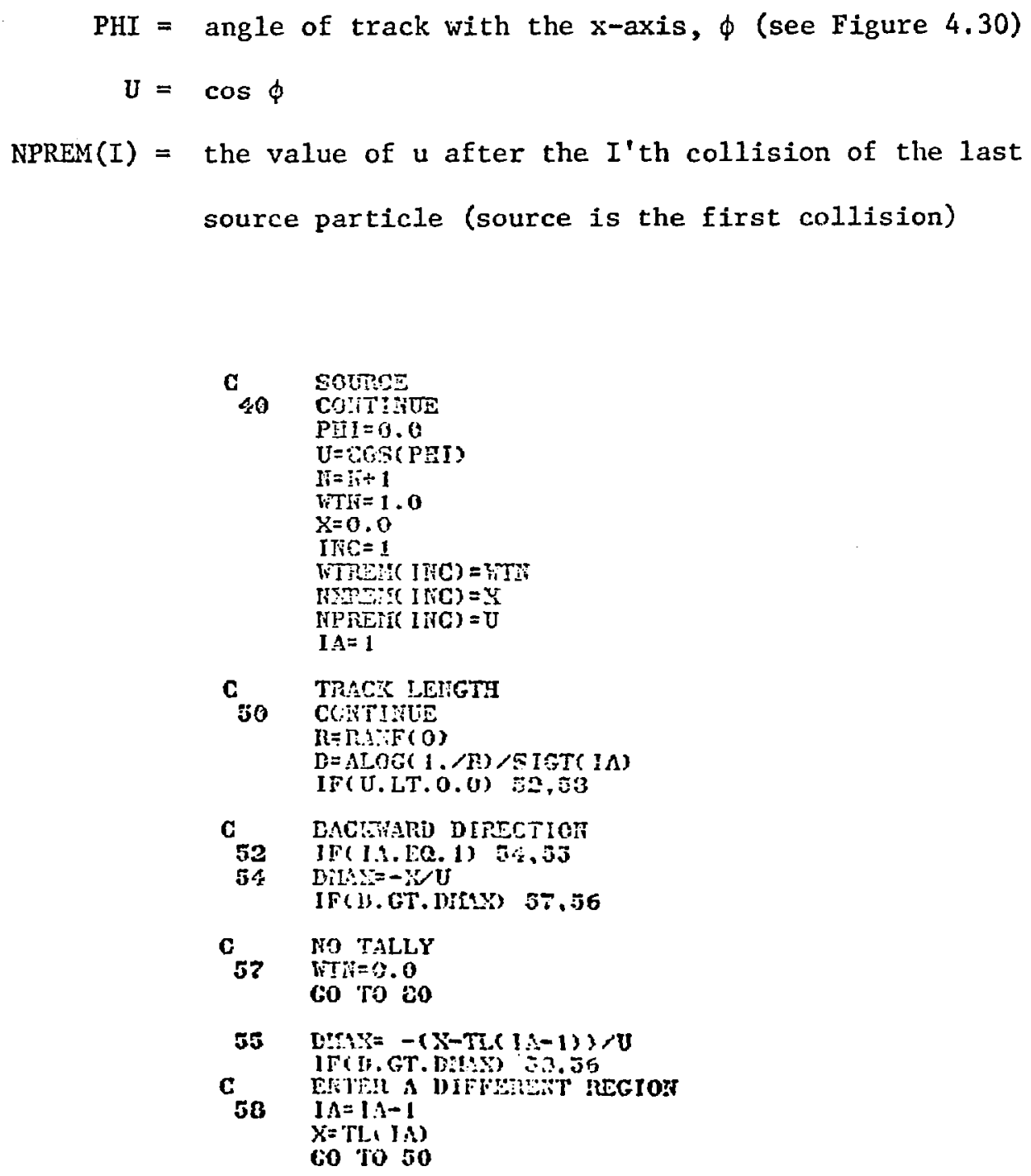




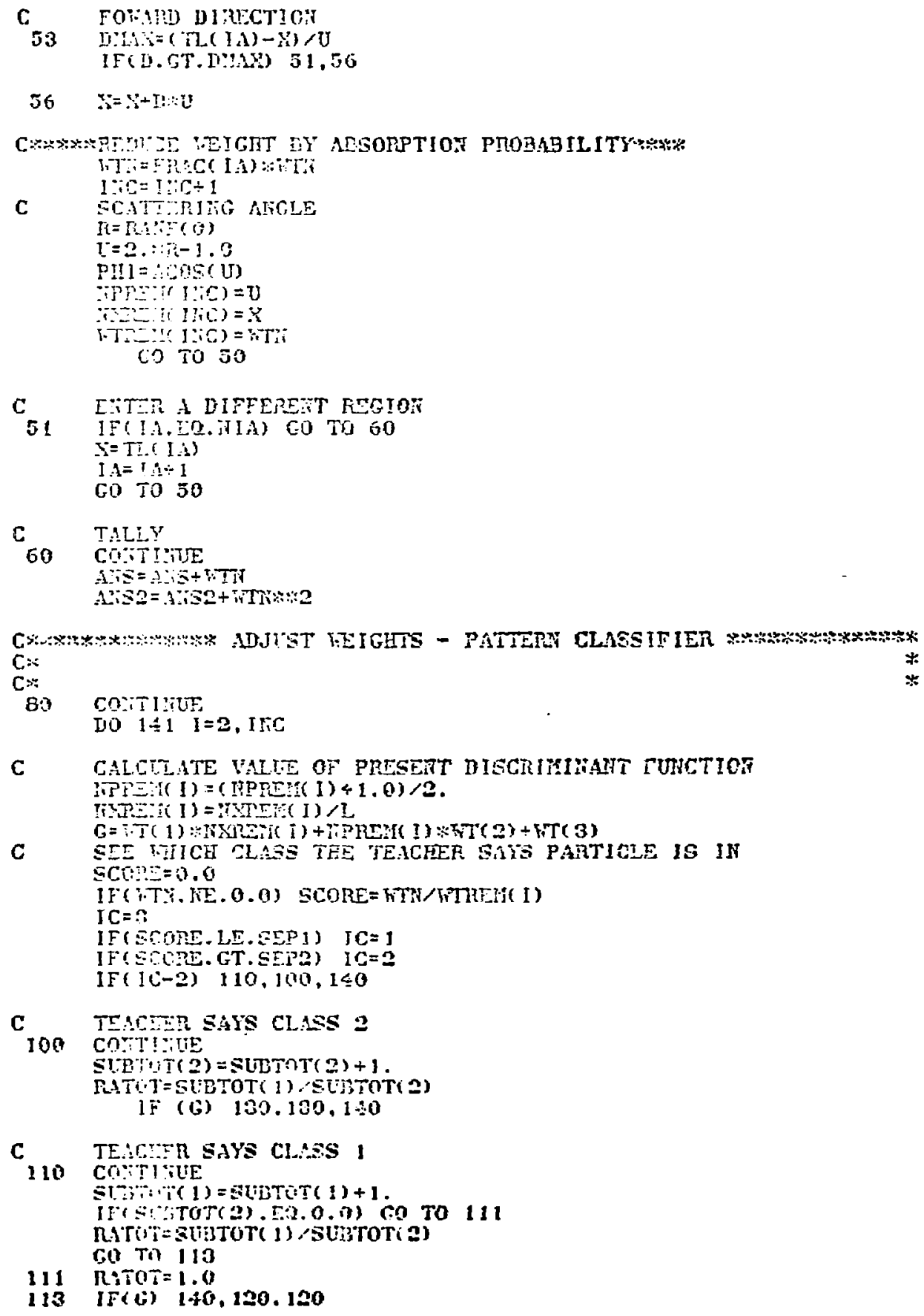

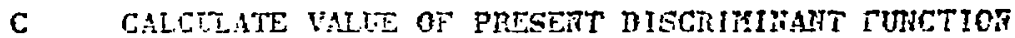

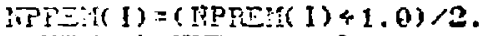




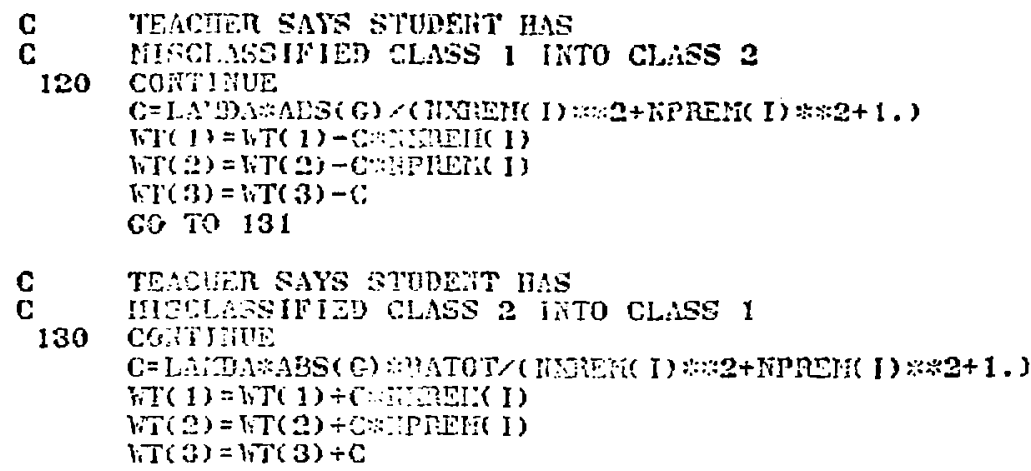

131 CCNTIIUVE

?

i. 1 COTTINUE

\section{C :}

$\mathrm{C} *$

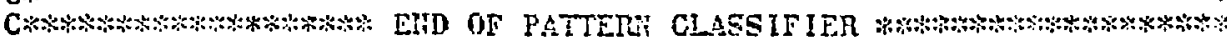

$\mathrm{RAT}=4 \mathrm{TS} / \mathrm{N}$

X2BAR $=A H S 2 / T$

C

ERROR CALCULATIOTS

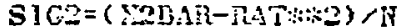

IE (SIG2. EO. -6.0$)$ SIG $=0.0$

IF(n.T. EO.0.0) Go To $1 \div 2$

TE=

142 IF(N.EN. FIIS) GO TO 181

131 Colitinte 
Appendix I: FORTRAN Coding for the Statistical Classifier with Two-Dimensional Pattern Space

The following computer listing has had all input - output and other extraneous coding removed. Parameters are defined as given in Appendices $\mathrm{H}, \mathrm{F}, \mathrm{D}$, and $\mathrm{C}$.

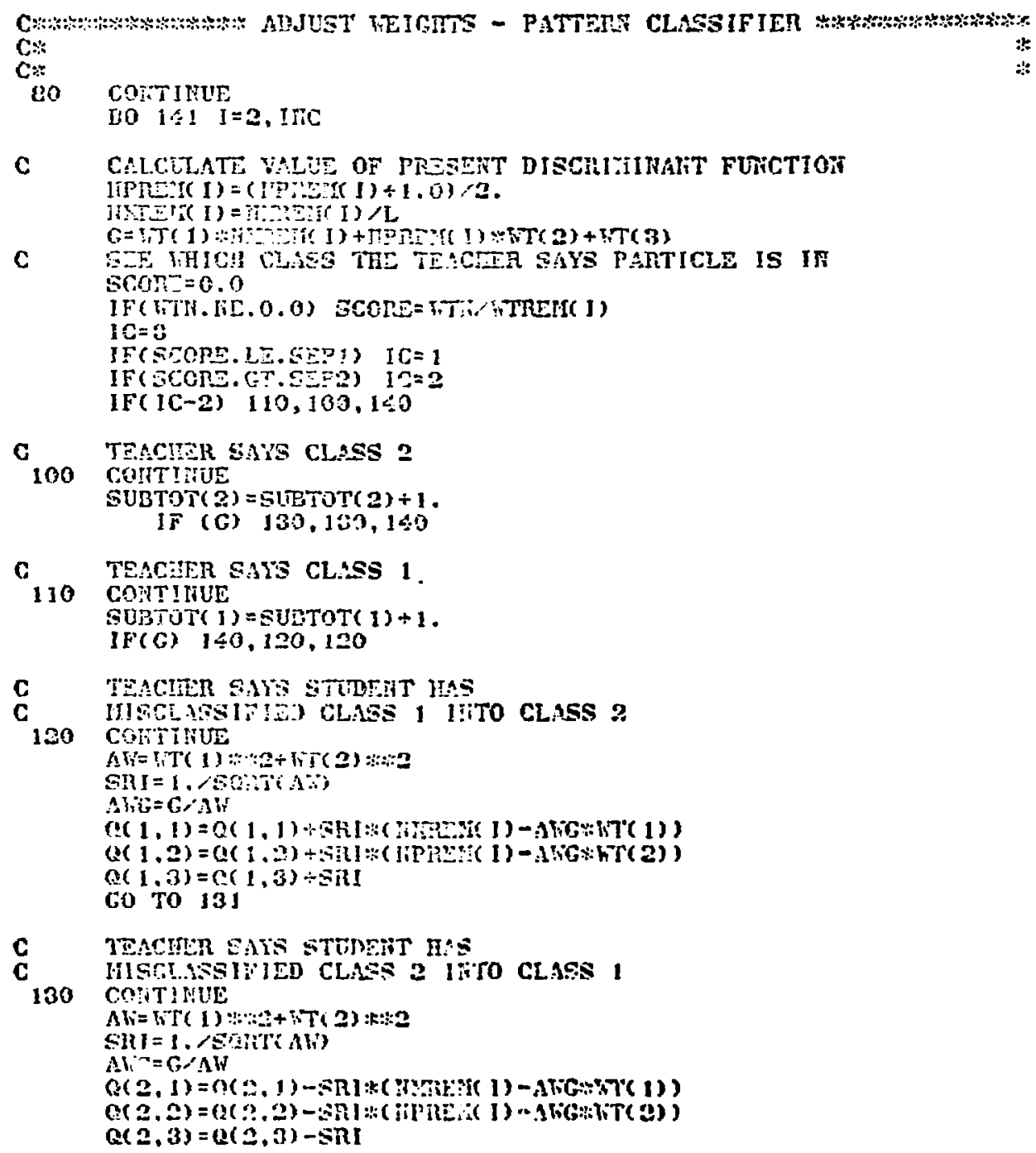




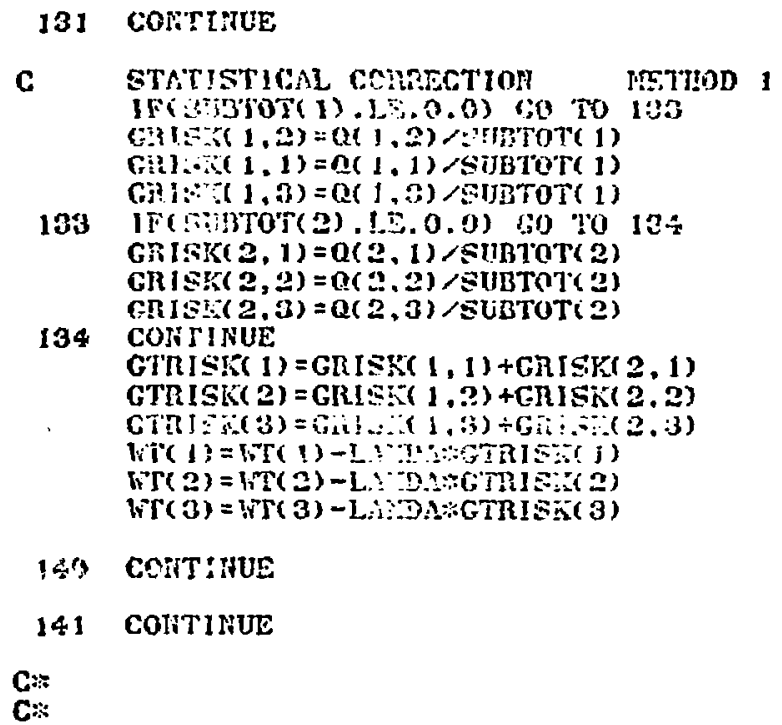

C.:

CAsts 
Appendix J: Geometry Description Used in the Neutron Monte Carlo Code MCN

This appendix contains a description of the geometry used in the MCN code at Los Alamos Scientific Laboratory. The code is designed to handle any number of first, second, and some fourth degree surfaces (limited only by computer storage) which divide the geometry of the problem into geometric cells (a cell is defined below).

If $f(x, y, z)=0$ is the equation of any surface in the problem, then for any arbitrary point in space $\left(\mathrm{x}_{0}, \mathrm{y}_{0}, \mathrm{z}_{0}\right)$, the sign of the quantity $f\left(x_{0}, y_{0}, z_{0}\right)$ is defined as the sense of the point $\left(x_{0}, y_{0}, z_{0}\right)$ with respect to the surface $f(x, y, z)=0$.

A geometric cel1 is defined such that:

(1) all points within a cell must have the same sense with respect to the bounding surfaces of that cell

(2) the senses of points within a cell must uniquely determine that cell from all other cells.

The cell corresponding to any point in space can be determined by comparing the senses of the point (with respect to the surfaces bounding each cell) to the senses that define each cell. The point belongs to the cell for which the senses agree. Cells do not necessarily correspond to material regions and are frequently specified (along with additional surfaces) for the purpose of splitting and Russian roulette. Each cell is assigned an importance which is used for splitting as described In Section 5.3.1. 


\section{APPENDIX $K$. DERIVATION OF $S_{f+1}$ FOR THE}

STATISTICAL CLASSIFIER AND LOSS $=d$

Let $s_{j}$ be the decision surface location as determined by

$$
s_{j}=\frac{-w_{2, j}}{w_{1, j}}
$$

where $w_{1, j}(1=1,2)$ is the $i$ 'th component of the $j$ 'th weight vector $w_{j}$. The first weight vector, $w_{1}$, is provided as an initial guess. Thereafter each $w_{j+1}$ is determined by (see Equation 3.16)

$$
W_{j+1}=W_{j}-\lambda \nabla \tilde{R}_{j+1}(W)
$$

where $\tilde{R}_{j}(W)$ is the $j$ 'th approximation to the risk (see Equation 3.19). The weight adjustment as given by Equation $\mathrm{K} .2$ is performed only after a prototype has been misclassified. Therefore

$$
j=\text { (total number of misclassified prototypes) }-1
$$

Using Equation 3.17 it follows that

$$
\nabla \tilde{R}_{j}(W)=\frac{1}{M_{1, j}} \sum_{n=1}^{N_{1} j}\left[\begin{array}{l}
\frac{\partial s\left(w_{n}, Y_{n}^{1}, c_{2} / c_{1}\right)}{\partial w_{1}} \\
\frac{\partial s\left(w_{n}, Y_{n}^{1}, c_{2} / c_{1}\right)}{\partial w_{2}}
\end{array}\right] \frac{1}{M_{2, j}} \sum_{n=1}^{N_{2, j}}\left[\begin{array}{l}
\frac{\partial s\left(w_{n}, Y_{n}^{2}, c_{1} / c_{2}\right)}{\partial w_{1}} \\
\frac{\partial s\left(w_{n}, Y_{n}^{1}, c_{1} / c_{2}\right)}{\partial w_{2}}
\end{array}\right](K .3)
$$


where $M_{i, j}$ and $N_{i, j}(i=1,2)$ are the number of prototypes and number of misclassified prototypes respectively of class $c_{i}$ after the $(j-1)^{\prime}$ th misclassification occurred $\left(N_{1, j}+N_{2, j}=j-1\right)$. Substituting the expressions for the partial derivatives of the loss function (see Table 4.4) into Equation $K .3$ results in

$$
\nabla \tilde{R}_{j}(w)=\left[\begin{array}{c}
\frac{1}{M_{1, j}} \sum_{n=1}^{N_{1, j}}\left(\frac{-w_{2, n}}{w_{1, n}^{2}}\right)+\frac{1}{M_{2, j}} \sum_{n=1}^{N_{2}}\left(\frac{w_{2, n}}{w_{1, n}}\right) \\
\frac{1}{M_{1, j}} \sum_{n=1}^{N}\left(\frac{1}{w_{1, n}}\right)+\frac{1}{M_{2, j}} \sum_{n=1}^{N_{2, j}}\left(\frac{-1}{w_{1, n}}\right)
\end{array}\right]
$$

where $w_{1, n}(i=1,2)$ is the $I^{\prime}$ th component of the $n^{\prime}$ th weight vector $W_{n}$ that existed when the $n^{\prime}$ th prototype of the corresponding class was misclassified.

Defining the vartables

$$
\begin{aligned}
& A_{1,1, j}=\frac{1}{M_{1, j}} \sum_{n=1}^{N}\left(\frac{-w_{2, n}}{w_{1, n}}\right) \\
& A_{2,1, j}=\frac{1}{M_{2, j}} \sum_{n=1}^{N_{2, j}}\left(\frac{w_{2, n}}{2}\right)
\end{aligned}
$$




$$
\begin{aligned}
& A_{1,2, j}=\frac{1}{M_{1, j}} \sum_{n=1}^{N_{1, j}}\left(\frac{1}{w_{1, n}}\right) \\
& A_{2,2, j}=\frac{1}{M_{2, j}} \sum_{n=1}^{N_{2, j}}\left(\frac{-1}{w_{1, n}}\right)
\end{aligned}
$$

Equation $K .4$ reduces to

$$
\begin{aligned}
& \nabla \tilde{R}_{j}(W)=\left[\begin{array}{l}
A_{1,1, j}+A_{2,1, j} \\
A_{1,2, j}+A_{2,2, j}
\end{array}\right]=\left[\begin{array}{l}
T_{1, j} \\
T_{2, j}
\end{array}\right] \\
& \text { where } T_{1, j}=A_{1,1, j}+A_{2,1, j} \\
& T_{2, j}=A_{1,2, j}+A_{2,2, j}
\end{aligned}
$$

Consider the case when the $j^{\prime}$ th misclassification consists of a prototype that has been misclassified from class $C_{1}$ into $C_{2}$. For this case

$$
\begin{aligned}
& A_{2,2, j+1}=A_{2,2, j} \\
& A_{2,1, j+1}=A_{2,1, j}
\end{aligned}
$$




$$
\begin{aligned}
& A_{1,1, j+1}=\frac{1}{M_{1, j}+1} \sum_{n=1}^{N_{1, j}+1}\left(\frac{-w_{2, n}}{w_{1, n}^{2}}\right) \\
& A_{1,2, j+1}=\frac{1}{M_{1, j}+1} \sum_{n=1}^{N_{1, j}+1}\left(\frac{1}{w_{1, n}}\right)
\end{aligned}
$$

Equation $\mathrm{K} .8 \mathrm{c}$ can be expanded resulting in

$$
\begin{aligned}
& A_{1,1, j+1}=\frac{1}{\left(M_{1, j}+1\right)}\left[\sum_{n=1}^{N_{1, j}}\left(\frac{-w_{2, n}}{w_{1, n}^{2}}\right)+\frac{-w_{2, p}}{w_{1, p}^{2}}\right] \\
& =\frac{1}{\left(M_{1, j}+1\right)}\left[M_{1, j} A_{1,1, j}+\frac{-w_{2, p}}{w_{1, p}^{2}}\right]
\end{aligned}
$$

where $p=N_{1, j}+1$. Similarly Equation $K .8 d$ becomes

$$
A_{1,2, j+1}=\frac{1}{\left(M_{1, j}+1\right)}\left[M_{1, j} A_{1,2, j}+\frac{1}{w_{1, p}}\right]
$$

Again assuming that a prototype belonging to $c_{1}$ has been misclassified into $C_{2}$, Equation $\mathrm{K} .7 \mathrm{a}$ becomes

$$
T_{1, j+1}=A_{1, I, j+1}+A_{2, I, j+1}
$$


Substituting Equations $\mathrm{K} .9$ and $\mathrm{K} .8 \mathrm{~b}$ into $\mathrm{K} .11$ results in

$$
T_{1, j+1}=\frac{1}{\left(M_{1, j}+1\right)}\left[M_{1, j} A_{1,1, j}+\frac{-w_{2, p}}{w_{1, p}^{2}}\right]+A_{2,1, j}
$$

However since

$$
w_{p}=w_{j}
$$

Equation $\mathrm{K} .12$ becomes

$$
T_{1, j+1}=\frac{1}{\left(M_{1, j}+1\right)}\left[M_{1, j} A_{1,1, j}+\frac{-w_{2, j}}{w_{1, j}^{2}}\right]+A_{2,1, j}
$$

Similarly $T_{2, j+1}$ is given by

$$
T_{2, j+1}=\frac{1}{\left(M_{1, j}+1\right)}\left[M_{1, j} A_{1,2, j}+\frac{1}{w_{1, j}}\right]+A_{2,2, j}
$$

Substituting Equation K.15 and K.14 into Equation K. 6 results in

$$
\nabla \tilde{R}_{j+1}(W)=\left[\begin{array}{l}
\frac{1}{\left(M_{1, j}+1\right)}\left(M_{1, j} A_{1,1, j}+\frac{-w_{2, j}}{2}\right)+A_{2,1, j} \\
\frac{1}{\left(M_{1, j}+1\right)}\left(M_{1, j} A_{1,2, j}+\frac{1}{w_{1, j}}\right)+A_{2,2, j}
\end{array}\right]
$$


Using Equation K.16 and Equation K.2 gives

$$
\begin{aligned}
& w_{1, j+1}=\left\{w_{1}-\lambda\left[\frac{1}{\left(M_{1}+1\right)}\left(M_{1} A_{1,1}+\frac{-w_{2}}{w_{1}^{2}}\right)+A_{2,1}\right]\right\}_{j} \quad \text { (K.17a) } \\
& w_{2, j+1}=\left\{w_{2}-\lambda\left[\frac{1}{\left(M_{1}+1\right)}\left(M_{1} A_{1,2}+\frac{1}{w_{1}}\right)+A_{2,2}\right]\right\}_{j} \quad(K .17 b)
\end{aligned}
$$

where the $j$ subscripts have been dropped on the right hand side of the equation. Using Equations $\mathrm{K} .17 \mathrm{a}$ and $\mathrm{K} .17 \mathrm{~b}$ in Equation $\mathrm{K} .1$ results in

$$
\begin{aligned}
s_{j+1} & =\frac{-w_{2, j+1}}{w_{1, j+1}}=\frac{-\left\{w_{2}\left(M_{1}+1\right)-\lambda\left[A_{1,2} M_{1}+\frac{1}{w_{1}}+A_{2,2}\left(M_{1}+1\right)\right]\right\}_{j}}{\left\{w_{1}\left(M_{1}+1\right)-\lambda\left[A_{11} M_{1}-\frac{w_{2}}{w_{1}^{2}}+A_{2,1}\left(M_{1}+1\right)\right]\right\}_{j}} \\
& =\frac{-\left\{w_{2}-\lambda\left[T_{2}-\frac{A_{1,2}}{\left(M_{1}+1\right)}+\frac{1}{w_{1}\left(M_{1}+1\right)}\right]\right\}_{j}}{\left\{w_{1}-\lambda\left[T_{1}-\frac{A_{1} 1}{\left(M_{1}+1\right)}-\frac{w_{2}}{w_{1}^{2}\left(M_{1}+1\right)}\right]\right\}_{j}} \\
& =\left\{\frac{s_{j} w_{1}+\lambda\left[T_{2}-\frac{A_{1}, 2}{\left(M_{1}+1\right)}+\frac{1}{w_{1}\left(M_{1}+1\right)}\right]}{w_{1}-\lambda\left[T_{1}-\frac{A_{1,1}}{\left(M_{1}+1\right)}+\frac{s_{j}}{w_{1}\left(M_{1}+1\right)}\right]}\right\}
\end{aligned}
$$


Equation K.18 is true only when a prototype belonging to $C_{1}$ has been misclassified. If a class $C_{2}$ prototype is misclassified, a similar treatment results in

$$
s_{j+1}=\left\{\frac{S_{j} w_{1}+\lambda\left[T_{2}-\frac{A_{2,2}}{\left(M_{2}+1\right)}-\frac{1}{w_{1}\left(M_{1}+1\right)}\right]}{w_{1}-\lambda\left[T_{1}-\frac{A_{2}, 1}{\left(M_{2}+1\right)}-\frac{S_{j}}{w_{1}\left(M_{2}+1\right)}\right]}\right\}_{j}
$$

In summary, if a prototype belonging to $C_{k}$ is misclassified, the next value of the decision surface is given by

$$
s_{j+1}=\left\{\frac{s_{j} w_{1}+\lambda\left[T_{2}-\frac{A_{k, 2}}{\left(M_{k}+1\right)}+\frac{(-1)^{k+1}}{w_{1}\left(M_{k}+1\right)}\right]}{w_{1}-\lambda\left[T_{1}-\frac{A_{k, 1}}{\left(M_{k+1}\right)}+\frac{(-1)^{k+1} s_{j}}{w_{1}\left(M_{k}+1\right)}\right]}\right]_{j}
$$

which is the desired result. 


\section{References}

1. J. M. Hammersley and D. C. Handscomb, Monte Carlo Methods, John Wiley \& Sons, Inc. (1964).

2. M. Clark and K. F. Hansen, Numerical Methods of Reactor Analysts, Academic Press (1964).

3. A. S. Householder, Ed., "Monte Carlo Method," Proceedings of a symposium held in June and July 1949, National Bureau of Standards Applied Mathematics Series, Vol. 12, issued June 11, 1951.

4. B. I. Spinrad et a1., "An Alignment Chart for Monte Carlo Solution of the Transport Problem," In Ref. 3.

5. G. W. Brown, "History of RAND's Random Digits - Sumary," In Ref. 3.

6. E. D. Cashwe11, Los Alamos Scientific Laboratory, personel communication, March 1975.

7. E. D. Cashwell and C. J. Everett, A Practical Manual on the Monte Carlo Method for Random Walk Problems, Permagon Press, New York (1959).

8. E. D. Cashwell et al., "Monte Carlo Code Development in Los Alamos," Presentation at the Monte Carlo Conference at Argonne National Laboratory, Argonne, Illinols, July 1-3, 1974. (Also available as LASL report LA-5903-MS).

9. J. Spanier, "A New Mult1-Stage Procedure for Systematic Variance Reduction in Monte Carlo," Proceedings of Conference on New Developments in Reactor Mathematics and Applications, CONF-710302, Pg. 760-770 (1971).

10. D. B. Mackillan, "Optimization of Importance-Sampling Parameters in Monte Car10," Nuclear Science and Engineering: 48, 219-231 (1972).

11. J. Spanier and E. M. Gelbard, Monte Carlo Principles and Neutron Transport Problems, Addison-Wesley, Massachusetts (1969).

12. R. Baldini-Celio et al., "A Multistage Self-Improving Monte Car10 Method," Nuclear Instruments and Methods 72 (1969), 317-320.

13. M. H. Welk, Standard Dictionary of Computers and Information Processing, Hayden Book Company (1970). 
14. L. L. Carter and F. D. Cashwe11, A Review of Particle Transport Simulation with the Monte Carlo Method to appear in the AFC Crit1cal Review Series, USAFC Technical Information Center, Oak Ridge, Tennessee.

15. N. J. N11sson, Learning Machines, McGraw-1111, Mew York (1965).

i6. H. C. Andrews, Introduction to Vathematical Teclintoues in Pattern Recognitjon, Wiley-Interscience, New York (1972).

17. W. Mendenha11 and R. L. Scheaffer, Mathematical Statistics with Applications, Duxbury Eress, Nassachusett, I973.

18. J. R. Lamargh, Introduction to Nuclear Reactor Theory, AddisonWesley Pub. Co. (1966).

19. E. D. Cashwe11, et a1., "MCN: A Neutron Monte Carlo Code." tos Alamos Scientific Laboratory report LA-4751 (1972).

20. L. L. Carter, "MCNA: A Computer Program to Solve the Adjoint Neutron Transfort Equation by Coupled Sampling wth the Yonte Carlo Method," Los Alamos Scientific Laboratory report IA-4488 (1971).

21. C. E. Burgart, "A General Method of Importance SampiIng the Angle of Scattering in Monte Carlo Calculations," Nuclear Sclence and Engineering: 42, 306-323 (1970).

22. K. Fukunaga, Introduction to Statistical Pattern Recognition, Academic Press, New York (1972).

23. K. S. Fu, Sequential Methods in Pattern Recognition and Machine Learning, Acadenic Press, New York (1968).

24. C. Chen, Statistical Pattern Recognition, Hayden Book Company, New Jersey.

25. T. Y. Young and T. W. Calvert, Classification, Estination and Pattern Recognition, American Elsevier Pub. Co., New York (1974).

26. R. L. Kashyap, "Algorithms for Pattern Classification," In Adaptive, Learning and Pattern Recognitfon Systems, J. M. Mendel and K. S. Fu, Eds.. Acadenic Press, New York, Chapter 3 (1970).

27. Y. C. Ho and A. K. Agrawala, "On Pattern Classification Algerithms - Introduction and Survey," Division of Engineering and Applied Physics Technical Report No. 557, Harvard University, March 1968. 
28. C. Mlaydon and Y. C. Ho, "On the Abatraction Problem in Pateern classificteion," Proc. Vutional Electron. Conf. (1966).

29. $Y$. A. Shreldex, Ed. The Monte Carlo Kethod, Permagon Pross, tew Vork (1966).

30. F. H. Clack, "The Fxponential TEaneforn as an Ioportance sapplinp Device, A Kevitu," Oak Ridge National laboratory (ORtL-RSIC-14) (1966).

31. S. Amarf, "A Theory of Adaptive Pactern Classiflers," IEEE Transactions on Flectronic Compters, Yol. EC-16, No. 3, PF. 299 (June 1967).

32. 1. H. Halos, ce 1. " "Autonarte Computation of Importance Sampling Functions for Monce Carlo Transpote Codes-Phase IXI, "DNA 2890F (1972).

33. E. D. Cashwe11, ec a1., "Wonte Carlo Phocon Codes: MCG and MCP." Los Alamos Sctentiffe Laboratory rapore LA-5157-YS (1973).

34. W. L. Thompson. "Gama-Ray and Flectron Transport by Monte Carlo," Doccoral Disearcation, Untuersiey of Virginta, Charlotecsulile. Mirginta (August 1974).

35. S. M. Selby, Ed. Standard yatheractcal Tables, Seventeenth Edteion, The Chemical Rubber Co*, Pg. 547 (1969).

36. A. Solew, "SECOND-Dlaped CP Tine for this Job," avallable from Los Alamos Scientific Laboratory progran 11brary, report $0110 \mathrm{~A}$ (March 1970).

37. B. Swartz, "Least Squares Polynomial Fitcing FoRTRAN IV Subroutine, with Coeffictents of Orthogonal and Legendre Polynoxials Mptional," avallable from los Alemos Scientific Laboratory program 11brary, report E208A (May 1967).

38. J. L. Macdorald, "Heuristic Learning Control for Nuclear Reactors," M.S. Thesis, Univereity of Texas at Austin (Auguse 1972).

39. B. L. Buzbee, "DotPRo - Inner Product of Two Vectors," ava1lable from Los Alamos Scientific laboratory program 11brary, report F124A (October 1972).

40. B. L. Buzbee, "ADDVEC - Vector Addition," available from Los Alamos Scientific Laboratory program 11brary, report F139A (Auguse 1973). 
41. C. J. Fverett and E. D. Cashwell, "A Monte Carlo Sampler," Los Alamos Sclentific Laboratory report LA-5061-MS (October 1972).

42. C. J. Everett and E. D. Cashwell, "A Second Monte Carlo Sampler," Los Alamos Sclentific Laboratory report LA-5723-MS (September 1974).

43. C. J. Everett, Los Alamos Sclentific Laboratory, personal comunications, March 1975. 